
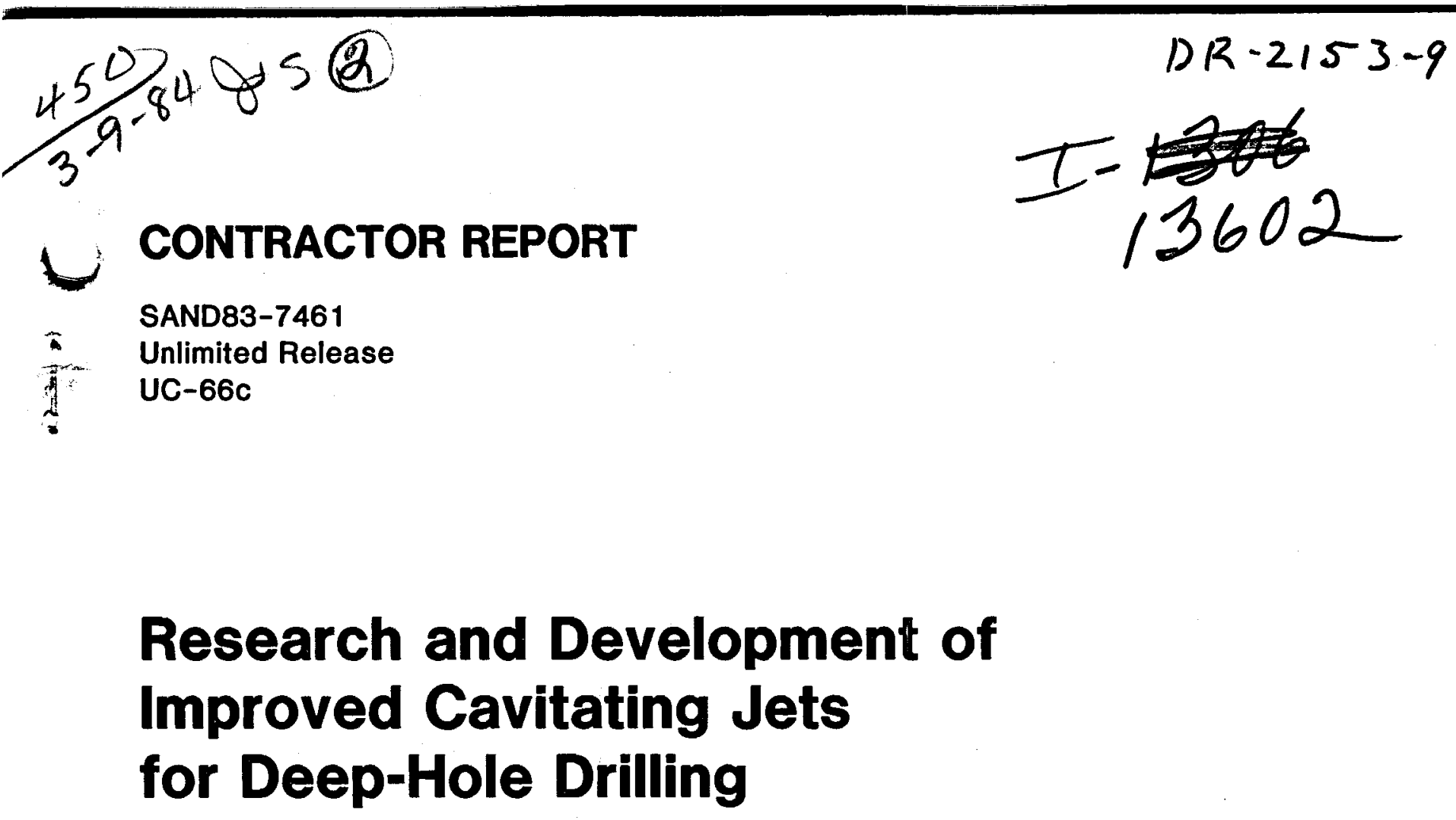

SAND83-7461

Unlimited Release

UC-66c

\title{
Research and Development of Improved Cavitating Jets for Deep-Hole Drilling
}

Virgil E. Johnson, Jr., William T. Lindenmuth, Georges L. Chahine, Andrew F. Conn, Gary S. Frederick

$\checkmark$ Hydronautics Inc.

7210 Pindell School Rd

$\checkmark$ Laurel, MD 20810

Prepared by Sandia National Laboratories Albuquerque, New Mexico 87185 and Livermore, California 94550 for the United States Department of Energy under Contract DE-AC04-76DP00789

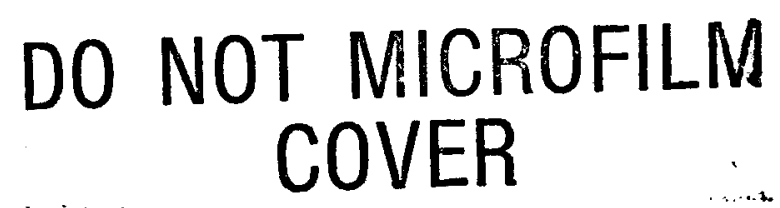

Printed January 1984

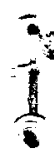




\section{DISCLAIMER}

This report was prepared as an account of work sponsored by an agency of the United States Government. Neither the United States Government nor any agency Thereof, nor any of their employees, makes any warranty, express or implied, or assumes any legal liability or responsibility for the accuracy, completeness, or usefulness of any information, apparatus, product, or process disclosed, or represents that its use would not infringe privately owned rights. Reference herein to any specific commercial product, process, or service by trade name, trademark, manufacturer, or otherwise does not necessarily constitute or imply its endorsement, recommendation, or favoring by the United States Government or any agency thereof. The views and opinions of authors expressed herein do not necessarily state or reflect those of the United States Government or any agency thereof. 


\section{DISCLAIMER}

Portions of this document may be illegible in electronic image products. Images are produced from the best available original document. 


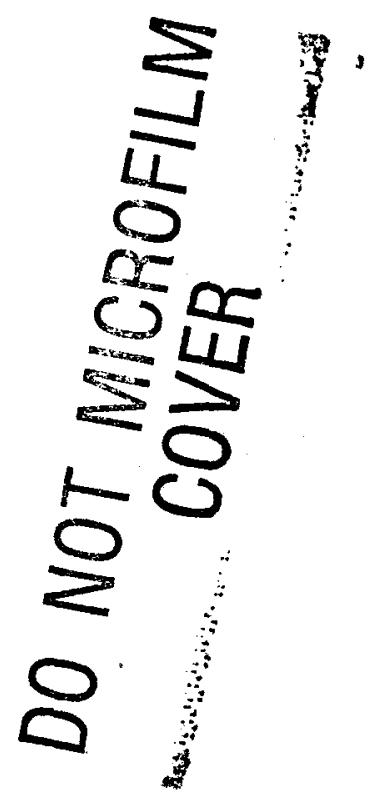

Issued by Sandia National Laboratories, operated for the United States Department of Energy by Sandia Corporation.

NOTICE: This report was prepared as an account of work sponsored by an agency of the United States Government. Neither the United States Government nor any agency thereof, nor any of their employees, nor any of their contractors, subcontractors, or their employees, makes any warranty, ezpress or implied, or assumes any legal liability or responsibility for the accuracy, completeness, or usefulness of amy information, apparatus, product, or process disclosed, or represents that its use would not infringe privately owned rights. Reference herein to any specific commercial product, process, or service by trade name, trademark, manufacturer, or otherwise, does not necessarily constitute or imply its endorsement, recommendation, or favoring by the United States Government, any agency thereof or any of their contractors or subcontractors The views and opinions expressed herein do not necessarily state or reflect those of the United States Government, any agency thereof or any of their contractors or subcontractors.

Printed in the United States of America Available from

National Technical Information Service

U.S. Department of Commerce

5285 Port Royal Roed

Springfield, VA 22161

NTIS price codes

Printed copy: A08

Microfiche copy: A01 


\title{
RESEARCH AND DEVELOPMENT OF \\ IMPJROVED CAVITATING JETS \\ FOR DEEP-HOLE DRILLING
}

by

\author{
Virgil E. Johnson, Jr., William, T: \\ Lindenmuth, Georges L. Chahine, \\ - Andrew F. Conn and \\ Gary s. Predeusin.
}

November 1982

\section{DISCLAIMER}

This report was prepared as an account of work sponsored by an agency of the United States Government. Neither the United States Government nor any agency thereof, nor any of their employees, makes any warranty, express or implied, or assumes any legal liability or responsibility for the accuracy, completeness, or usefulness of any information, apparatus, product, or process disclosed, or represents that its use would not infringe privately owned rights. Reference herein to any specific commercial product, process, or service by trade name, trademark, manufacturer, or otherwise does not necessarily constitute or imply its endorsement, recommendation, or favoring by the United States Government or any agency thereof. The views and opinions of authors expressed herein do not necessarily state or reflect those of the United States Government or any agency thereof.

\section{Prepared for}

Sandia Laboratories

Geothermal Well Technology Division

Albuquerque, New Mexico 87115

Under

Contract No. 13-5129 


\begin{abstract}
Improved cavitating nozzles have been developed as part of an on-going program to increase the rate of penetration of deephole drill bits. Based on the four criteria of: incipient cavitation number, amplitude of pressure fluctuation (and hence enhanced structuring of the jet flow), rock cutting, and cleaning chips from the hole bottom - these new, "STRATOJET" (STRuctured Acoustically Tuned oscillating JET) cavitating nozzle systems have out-performed both conventional drill bit nozzles and the basic CAVIJET cavitating jets. Although nozzle designs which provide large amplitude pressure modulations are now available for the operation in water, additional research is needed to optimize self-resonating jets for use: (a) in mud, (b) in specific drill bit designs; and (c) at higher system pressures than now currently used for deep-hole drilling.
\end{abstract}


FOREWORD

The study described in this report was conducted by HYDRONAUTICS, Incorporated, Howard County, Laure1, Maryland. Portions. of the experimental program were conducted under subcontracts, within the Drilling Research Laboratory (DRI) at Terra Tek, Inc., Salt Lake City, Utah; and in the laboratory of Professor Albert T. Ellis, University of California, La Jolla, California. This program was supported by the U,S. Department of Energy, Division of Geothermal Energy, under Sandia Laboratories Contract No. 135129 ; and portions of the costs were shared by NL/HYCALOG/NL Industries and SMITH TOOL, Division of Smith International, Inc. Specific contributions from NL/HYCALOG included design and engineering support, the CAVIJETه cavitating fluid jet nozzles and some of the tooling and bits used in tests at DRI, as well as a portion of the DRL rentals. Funds for the development of nozzle designs for specific bits were also provided by NL/HYCALOG. SMITH TOOL furnished the roller bits for the DRL tests, plus design and engineering support and a portion of the DRI rentals.

The CAVIJET cavitating fluid jet technology used in this program has been patented by HYDRONAUTICS, Incorporated and additional patent applications related to the tuned-resonator STRATOJET have been submitted. The CAVIJET technology is currently being developed for a variety of commercial applications.

* U.S. Patent Nos, $3,528,704 ; 3,713,699 ; 3,807,632$; and $4,262,757$. Other U.S, and Foreign patents are pending or have been granted. CAVIJET is a registered trademark of HYDRONAUTICS, Incorporated. 


\section{ACKNOWLEDGMENTS}

The following organizations and individuals have made important contributions to the success of this phase of our investigations. The authors would like to express their sincere appreciation to:

- Sandia Laboratories - Dr. James R. Kelsey and Mr. David A. Glowka, for their support, advice, and encouragement throughout this program; and Dr. Samuel G. Varnado, now with NL/Industries, for his assistance during the initial portions of our study.

- NL/HYCALOG - Mr. George J. Giacchino, Jr., whose support and guidance have kept this study on course for many years. We also thank Mr. William Short, Jr., and Mr. John Sherrill for their help in planning, arranging, and conducting the DRL nozzle and bit tests, and Mr. Robert P. Radtke, now with the STRATA BIT Corporation, whose efforts and enthusiasm were essential in the creation and advancement of this program.

- SMITH TOOL - Mr. William Baker and Mr. Scott Forrest, for continued inputs to both the laboratory and field trials of bits, and their strong moral support for our program.

- University of California, San Diego - Professor Albert T. Eliis for his many creative efforts during this phase of the program. His work included consultation and a variety of new experimental tests related to flow and pressure distributions created by structured jets, and photographic procedures, which served as the basis for our more extensive efforts.

- Terra Tek/Drilling Research Laboratory - As always, the staff at DRL provided us the finest and most professional support possible; we particularly thank Mr. Alan D. Black and Mr. John Sandstrom for their efforts during those very difficult tests.

- HYDRONAUTICS, Incorporated - Mr. Phillipe Genoux, Mr. Daniel F. Dementhon, and Mr. George E. Matusky, for contributions to various experimental and analytical tasks. 
TABLE OF CONTENTS

1.0 INTRODUCTION ................. 1

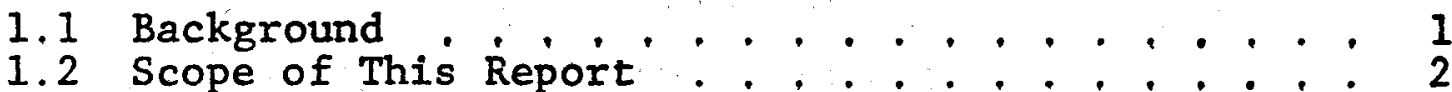

2.0 CAVITATION INCEPTION STUDIES .......... 5

2.1 Preliminary Inception Experiments . . . . . . 5

2.1.1 Plain CAVIJET nozzles . . . . . . . 6

2.1.2 CAVIJET nozzles with central devices. . . 7

2.1.3 PULSER CAVIJET nozzle system....... 9

2.1.4 ORGAN-PIPE CAVIJET nozzle system . . . . . 9

2.1.5 PULSER-FED CAVIJET nozzle system . . . . 10

2.1.6 Summary and conclusions of preliminary inception tests . . . . . . . . . . 10

2.2 Vortex Generating Nozzles

2.2.1 Theory . . . . . . . . . . . . II

2.2.2 Experimentai studies . ... . . 19

3.0 ORGAN PIPE ACOUSTICS THEORY . . . . . . . . . 23

3.1 Single Pipe . . . . . . . . . . 23

3.2 Single-Pipe Modes and Optimum Mach Number . . . 28

3.3 Case of a Series Pipe System ........ 31

4.0 ROCK EROSION TESTS ............... . . 35

4.1 Sierra White Granite Tests at DRL . . . . . . 35

4.1.1 Test Parameters . . . . . . . . . 37

4.1.2 Nozzle Wear ............ 38

4.1.3 Specimen Calibrations . . . . . . 38

4.1.4 Erosion Rates ............ 40

4.2 Tests on Sedimentary Rocks ........... 41

4.2.1. Calibration of sandstone specimens.... 41

4.2.2 Test Results........... 45 
TABLE OF CONTENTS - Continued

Page

No.

5.0 CHIP LIFTING TESTS . . . . . . . . . . . . . 51

5.1. Chip Hold-Down Facility . . . . . . . . . 51

5.2 Mud Bed Erosion . . . . . . 52

5.3 Modulated Pressure Distributions . . . . 53

6.0 POLYCRYSTALLINE DIAMOND COMPACT (PDC) BIT TESTS ... 57

6.1 Bit Test Parameters . . . . . . . . . . . 57

6.2 Test Results . . . . . . . . . . 59

6.2 .1 Wear-in . . . . . . . . . . . 59

6.2 .2 Bore hole pressure . . . . . . . . . 60

6.2 .3 RPM .................. 61

6.2 .4 Bit weight . . . . . . . . . . . . 61

6.2 .5 Hydraulic power . . . . . . . . . . 61

6.3 Summary of Results . . . . . . . . . . . 61

7.0 THREE-CONE BIT TESTS . . . . . . . . . . . . . 63

7.1 Bit Test Parameters... . . . . . . . . 63

7.2 Pressure Fluctuation Test Results . . . . . .65

7.2 .1 Water ............ . . 67

7.2.2 Drilling Mud............. 68

7.3 Bit Hydraulics Analysis . . . . . . . . . 69

7.4 Drilling Test Results . . . . . . . . . . 71

7.4 .1 WOB ................71

7.4 .2 RPM ................ . . 71

$7.4 .3 \mathrm{p}_{\mathrm{a}}$ and $\Delta \mathrm{p}$

7.4.4 Modulation and Drilling Rate. . . . . . . 72

7.5 Conclusions from DRL Tests with Three-Cone Bits . 72

8.0 CONCLUSIONS AND RECOMMENDATIONS . . . . . . . . 75

REFERENCES . . . . . . . . . . . . . . 77 
APPENDIX A - "Cavitating and Structured Jets for Mechanical Bits to Increase Drilling Rate"

APPENDIX B' - "Self-Resonating Cavitating Jets".

APPENDIX C - "The Development of Structured Cavitating Jets for Deep-Hole Bits" 


\section{LIST OF FIGURES}

FIGURE 1 - Approximate Incipient Cavitation Numbers for CAVIJET ${ }^{\circledR}$ and Other Nozzle Types (Acoustic Detection)

FIGURE 2 - Experimental Incipient Cavitation Numbers for Vortex and Centerbody CAVIJET (8) Nozzles; $\sigma_{i}, 0$ is incipient cavitation number for plain CAVIJETi nozzle

FIGURE 3 - Cavitation Pattern for Vortex (ORGAN-PIPE CAVIJETه) Nozzle; $\sigma=0.25, d=6.4 \mathrm{~mm}$ (0.25 in.), $D=12.7 \mathrm{~mm}$ (0.50 in.), $x=22.3 \mathrm{~mm}(0.88$ in.) (see FIGURE 2)

FIGURE 4 - Experimental Incipient Cavitation Number for PULSER CAVIJET Nozzle; $\sigma^{\circledR} \circ$ is incipient cavitation number for plain CAVIJET nózzle

FIGURE 5 - Experimental Incipient Cavitation Numbers for ORGANPIPE CAVIJET Nozzle; $\sigma_{i, 0}$ is incipient cavitation number for plain CAVIJET' nozzle

FIGURE 6 - Experimental Incipient Cavitation Numbers for PULSERFED CAVIJET Nozzle; $\sigma_{i} 0$ is incipient cavitation number for plain CAVIJET nozzle

FIGURE 7 - Experimental Incipient Cavitation Numbers for Various CAVIJET ${ }^{8}$ nozzle types; $\sigma_{i}$, is incipient cavitation number for plain CAVIJET nozzle

FIGURE 8 - Parameters for Vortex Nozzle Analysis

FIGURE 9 - Distribution of Tangential Velocity and Pressure Associated with a Vortex

FIGURE 10 - Large Scale Nozzle Installation in the High Speed Channel

FIGURE 11 - Submerged Jet Cavitation as Observed by Long Duration Exposure; photograph taken in High Speed Channel

FIGURE 12 - Cavitation as Observed by Short Duration Exposure, Showing Jet Structuring; photograph taken in High Speed Channe1 
FIGURE 13 - Segments of Rotating Cavitating Vortices At Periphery of Jet; photograph taken in High Speed Channel

FIGLRE 14 - Ring Vortex in Submerged Jet Flow; randomly generated during tests in High Speed Channel

FIGURE 15 - Tip Vortex Generating Foils, with constant span and swept shape

FIGURE 16 - Tip Vortex Generating Foils, with variable span and flat shape

FIGURE 17 - High Speed Channel Cavitation Inception

FIGURE 18 - Typical Extended Nozzle Feed-Tube for Three-Cone Roller Bit

FIGURE 19 - Parameters for Analysis of ORGAN-PIPE Acoustics

FIGURE 20 - Correlation of Optimum Mach Numbers and Geometry of ORGAN-PIPE CAVIJET Nozzles

FIGURE 21 - ORGAN PIPE with Series of Different Cross Sections

FIGURE 22 - ORGAN PIPE with Two Cross Section Areas

FIGURE 23 - Graphical Solution for the Resonance of a Two-step ORGAN PIPE

FIGURE 24 - Nozzles Used in Granite Tests at DRL

FIGLRE 25 - Configuration for Stationary Nozzle Tests at DRL

FIGURE 26 - Erosion Rates for $\Delta \mathrm{p}=27.6 \mathrm{MPa}$ (4 ksi)

FIGURE 27 - Erosion Rates for $\triangle \mathrm{p}=34.5 \mathrm{MPa}$ (5 ksi)

FIGURE 28 - Effect of Nozzle Pressure on Erosion Rate

FIGURE $29^{\circ}$ - Configurations of ORGAN-PIPE Nozzles Used in Rock Cutting Tests (See Figure 30)

FIGURE 30 - Comparison of Slots Cut by an ORGAN-PIPE Cavitating Jet and a Conventional Jet 
-viii-

\section{LIST OF FIGLRES - Continued}

FIGURE 31 - Half-Scale Nozzles Used in Comparative Rock Cutting Tests

FIGURE 32 - Comparative Erosivity of a PULSER and a plain CAVIJET

FIGURE 33 - Comparative Erosivity of a Plain and a PULSER-FED CAVIJET

FIGURE 34 - Basic Plain CAVIJET Size Series

FIGURE 35 - Effect of Size and Standoff on Slot Cutting Penetration for Plain CAVIJET, Indiana Limestone

FIGURE 36 - Effect of Size and Standoff on Slot Cutting Penetration for Plain CAVIJET, Berea Sandstone

FIGURE 37 - New, Low Pressure, Chip Hold-Down Test Loop

FIGURE $3 \delta$ - Clear Acrylic Test Chamber in Chip Hold-Down Facility

FIGURE 39 - Pressure Transducers in Porous Metal Support Plate of Chip Hold-Down Facility

FIGURE 40 - Ring Vortices Spreading on Surface at $X / d=2.0$, $\mathrm{S}_{\mathrm{d}}=0.64$

FIGURE 41 - Comparison of Mud bed Penetration for Unstructured and Structured Jets, $5 \mu$ Cloth

FIGURE 42 - Comparison of Mud Bed Penetration for Unstructured and Structured Jets, $30_{\mu}$ Cloth

FIGURE 43 - Pressure Distribution on Target Plate: for standoff: $\mathrm{X} / \mathrm{d}=1.2$

FIGURE 44 - Pressure Distribution on Target Plate: for standoff: $\mathrm{X} / \mathrm{d}=2.9$

FIGURE 45 - Nozzles for Experimental $21.6 \mathrm{~cm}$ ( $8 \frac{3}{2}$ in.) PDC Bits

FICURE 46 - Experimental $21.6 \mathrm{~cm}$ ( $8 \frac{1}{2}$ in.) PDC Bits

FIGURE 47 - Wear-in of PDC Bit During DRL Tests; plain CAVIJET Nozzles 


$$
-\mathbf{i x -}
$$

\section{LIST OF FIGURES - Continued}

FIGURE 48 - Wear-in of PDC Bit During DRL Tests; Standard Drill Bit Nozzle

FIGURE 49 - Wear-in of PDC Bit During DRL Tests; PULSER CAVIJET Nozzles

FIGURE 50 - Effect of Bore Hole Pressure on PDC Bit Penetration

FIGURE 51 - Comparison of Drill Bit Performance in Mancos Shale

FIGURE 52 - Modifications to Tubes for ORGAN PIPE CAVIJET Nozzles in Three-Cone Bit

FIGURE 53 - Pressure Modulations in Water

FIGURE 54 - Pressure Modulations in $1.14 \mathrm{gm} / \mathrm{cm}^{3}$ (9.5 ppg) Mud

FIGURE 55 - Pressure Modulation Amplitudes in Water and Mud

FIGURE 56 - Discharge Coefficient for Center Venturi Jet

FIGURE 57 - Least Square Fit to Modified Bit Discharge Through Extended Tube Nozzles

FIGURE 58 - Effect of Weight on Bit on Drilling Rate

FIGURE 59 - Effect of RPM on Drilling Rate

FIGURE 60 - Relative Drilling Performance vs. Bore Hole Pressure

FIGURE 61 - Effect of Modulation Amplitude on Relative Drilling Rate 


\section{$-\mathbf{x}-$ \\ LIST OF TABLES}

Page

No.

TABLE 1 - Some Typical End Impedances . . . . . . . 26

TABLE 2 - Slot Cutting Comparisons , . . . . . , 47

TABLE 3 - Comparison of Relative Penetration of Rock For Some Resonating Nozzle Types Tested (Relative to Basic CAVIJET( ). . . . . . . . . . . 49

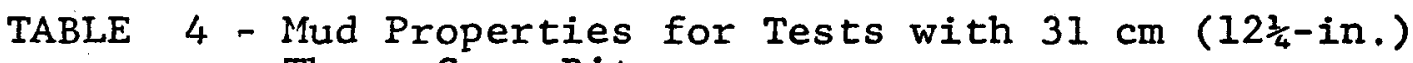
Three-Cone Bits...... . . . . . . 66 
$-x i-$

SUMMARY

Improved cavitating nozzles have been developed as part of an on-going program to increase the rate of penetration of deephole drill bits. Based on the four criteria of: incipient cavitation number, amplitude of pressure fluctuation (and hence enhanced structuring of the jet flow), rock cutting, and cleaning chips from the hole bottom - these new, self-resonating cavitating jets have out-performed both conventional drill bit nozzles and the basic CAVIJET ${ }^{\circledR}$ cavitating jets. Tests were conducted with individual nozzles, at full scale, and with smaller scale and very large scale models, using air, water, and drilling mud as the working fluid. Full-scale drill bits were also tested in these three fluids. Analytical tools have been developed for predicting the performance of ORGAN-PIPE CAVIJET nozzles and central-vortex generating nozzles.

Some of the conclusions drawn from this phase of the program are :

1. Several self-resonating nozzles systems*, the PULSER, PULSER-FED, and ORGAN-PIPE CAVIJET designs produce passive oscillations which (a) cause the jet to structure into discrete ring vortices, and thus (b) provide nozzle systems which have incipient cavitation numbers two to six times higher than obtained with either conventional CAVIJET nozzles or typical drill bit nozzles.

2. In rock cutting trials, higher incipient cavitation numbers have been correlated with greater erosivity.

* An acronym: "STRATOJET" (STRuctured Acoustically Tuned Qscillating JET) will be used for these systems. 
-xii-

3. The ORGAN-PIPE CAVIJET system is adaptable to the physical restraints of existing bits, and is the most promising concept for future bit designs.

4. STRATOJET nozzle systems will clean chips from a simulated hole bottom at a nozzle pressure drop only about 25 percent of that required for standard nozzle systems.

5. Self-resonating structured jets can be achieved either with or without cavitation. Therefore, at hole depths below where cavitation is present, a STRATOJET nozzle may still contribute to improved bottom hole cleaning.

Although nozzle designs which provide large amplitude pressure modulations are now available for operation in water, additional research is needed to optimize self-resonating jets for use: (a) in mud, (b) in specific drill bit designs, and (c) at higher system pressures than now currently used for deep-hole drilling. 


\subsection{INTRODUCTION}

\subsection{Background}

The studies described in this report are part of an ongoing effort to understand and develop cavitating jets which will enhance the performance of bits used to drill for geothermal or fossil energy. During the first phase (Reference 1) we found that the existing CAVIJET cavitating fluid jet nozzle designs were capable of operating under certain, limited downhole conditions, and improved rates of penetration (ROP) were observed with two-cone roller bits during both laboratory and field trials. Although these existing designs, now termed "conventional" or "basic" CAVIJET nozzles, gave encouraging results, the limits on their practical use motivated the study described in Reference 2 .

Conventional CAVIJET nozzles were capable of producing cavitation, using drilling mud, to a depth of about $1,200 \mathrm{~m}$ (4,000 ft) when operated at a nozzle pressure drop, $\Delta p$, of about $13.8 \mathrm{MPa}(2,000 \mathrm{psi})$. Due to the rather large size of these nozzles, however, necessitated by their diameter contraction ratio, $D / d$, of four (see Section 2.1 for a discussion of this factor), the conventional CAVIJETS could only be fitted within a two-cone roller bit, a bit type which is seldom used in comparison to the widely accepted three-cone roller bit. Thus, the study summarized in Reference 2 had the objective of discovering whether principles of "self-resonance" could be developed for improving the performance of submerged cavitating jets - thus eventually leading to new nozzle systems which are: (i) more erosive, (ii) capable of providing increased ROP at greater depths than conventional CAVIJET nozzles, and (iii) small enough to fit into the confines of existing three-cone 
roller bits with minimal modifications to the extended nozzle tubes now used in these drill bits.

The results from this feasibility study demonstrated that self-resonating cavitating jet systems were indeed capable of providing the required performance for use in deep drilling three-cone bits. Specifically: (i) several passive resonating concepts were identified and tested; each provided structuring of the jet cavitation into well-defined ring vortices, (ii) the structured, flows from these jets showed incipient cavitation numbers larger than conventional jets, thus providing cavitation erosion at greater depths, (iii) under comparable conditions, preliminary rock cutting tests in water showed that a resonating jet was more erosive than a conventional cavitating jet, and (iv) preliminary versions of these new nozzle system concepts suggested that designs could be developed which would fit into three-cone bits, and within new bits which use PDC (polycrystalline diamond compact) cutting elements. These results, and the recommendations developed during that phase of the program, led to the effort described in the present report.

\subsection{Scope of This Report}

During the course of this investigation a number of papers describing portions of our cavitating jet research and development have been presented at technical conferences. Three of these papers (References 3, 4,5), contain detailed discussions of certain tasks that were completed under the contract which this report is summarizing - therefore, these three papers are reprinted herein as Appendices $A, B$, and $C$. The reader is advised to read through these appendices before proceeding with subsequent sections in the main body of this report since considerable background information as well as details from prior and present research are contained in these papers. To insure 
that the efforts under each task in the scope of work for this contract were fully described, it was sometimes necessary in the main text to duplicate information given in one of the appendices - we beg the reader's indulgence of this occasional repetition.

The following guide is provided, to facilitate location of each aspect of this phase of the program:

- Effects of jet structuring on cavitation inception: Appendix A, pp. 5-7.

- Self-resonating nozzle design concepts: Appendix B, pp. 6-7.

- Cavitation inception

Preliminary studies: Main text, Section 2.1.

Study of vortex generating nozzles: Main text, Section 2.2.

- Organ pipe acoustics theory: Main text, Section 3.0; Appendix A, Pp. 8-9.

- Optimization of nozzle parameters affecting resonance (tests in air and in water): Appendix A, PP. 9-12, Appendix C, pp. 3-4.

- Rock cutting, with individual nozzles: Main text, Section 4.0; Appendix A, PP. 12-14.

- Hole bottom cleaning: Main text, Section 5.0; Appendix A, pp. 7-8; Appendix C, Pp. 4-5.

- Drill bit tests

PDC bits: Main text, Section 6.0.

Three-cone roller bits: Main text, Section 7.0. Two-cone roller bits: Appendix A, pp. 4-5. 


\subsection{CAVITATION INCEPTION STUDIES}

As summarized in the Introduction and discussed in detail in References 1 and 2 , cavitating jets have been shown to be feasible for improving the drilling rate of bits used in deep hole drilling. Since nozzles having higher values of the incipient cavitation number will remain influenced by the cavitation effect to greater drilling depths or (for the same operating cavitation number) will have a greater cavitation effect, our research and development has been directed toward finding nozzles with higher values of the incipient cavitation number.

\subsection{Preliminary Inception Experiments}

The present study started with cavitation inception experiments on all of the various forms of CAVIJET nozzles and nozzle systems described in References 1 and 2 so as to form a base of information from which the most promising nozzles could be selected for further development. The types of CAVIJET nozzle systems studied were:

- Basic CAVIJET nozzle contour with various contraction ratios (a nonresonating CAVIJET nozzle, with no inserts to stimulate cavitation is termed a "plain" CAVIJET).

- Basic CAVIJET nozzles with center bodies or central vortex generators.

- PULSER CAVIJET nozzle systems

- PULSER-FED CAVIJET nozzle systems

- ORGAN-PIPE CAVIJET nozzle systems.

Since the minimum pressure in the flow field and thus the incipient cavitation number, $\sigma_{i}$, for any cavitating jet is influenced by viscosity, the incipient cavitation number was 
determined for all nozzle types over a range of Reynolds numbers*, $R_{e}=v d / v$.

The preliminary cavitation experiments were carried out in the High Pressure Cell Facility described in References 1 and 2 (also, see Appendix A, p. 10). Incipient and desinent** cavitation was determined both visually and acoustically for a range of pressure drops across the nozzles up to approximately $6.9 \mathrm{MPa}(1,000 \mathrm{psi})$. The visually determined value of $\sigma_{i}$ was usually about 15 to 20 percent lower than that determined by the transducer located in the test chamber. There was considerable scatter in the data $( \pm 25 \%)$ and only faired results which represent the best average of many observations are presented.

2.1.1 Plain CAVIJET nozzles - Figure 1 shows a comparison of the measured incipient cavitation number for plain CAVIJET nozzles having diameter contraction ratios of $D / d \geq 4$ and 2 , with typical deep hole drill bit nozzles ( $D / d \simeq 2$ to 3 ) and a long nozzle such as the Leach and Walker nozzle described in Reference 1. The important points to note in Figure 1 are that the plain CAVIJET with $\mathrm{D} / \mathrm{d} \geq 4$ has an incipient cavitation number (at $R_{e}=10^{6}$ which is typical of drill bit applications) of 1.8 or about twice that of typical drill bit nozzles and about four times that of long nozzles. However, if the contraction ratio of the basic CAVIJET nozzle is reduced to two, the incipient cavitation number is approximately the same as that of typical drill bit nozzles. It is this important requirement of the conventional CAVIJET nozzle to have a contraction ratio of four or greater which limits its application. As pointed out in Reference 1, only the two cone roller bit has adequate space to permit such large contraction ratios and it was for this reason that the Smith $A-1$ two cone bit was selected to test the basic CAVIJET in the field.

* See Nomenclature on p. 2 of Appendix B.

** Inception refers to the onset of cavitation; desinence is the cessation of cavitation, as when pressure is increased. 


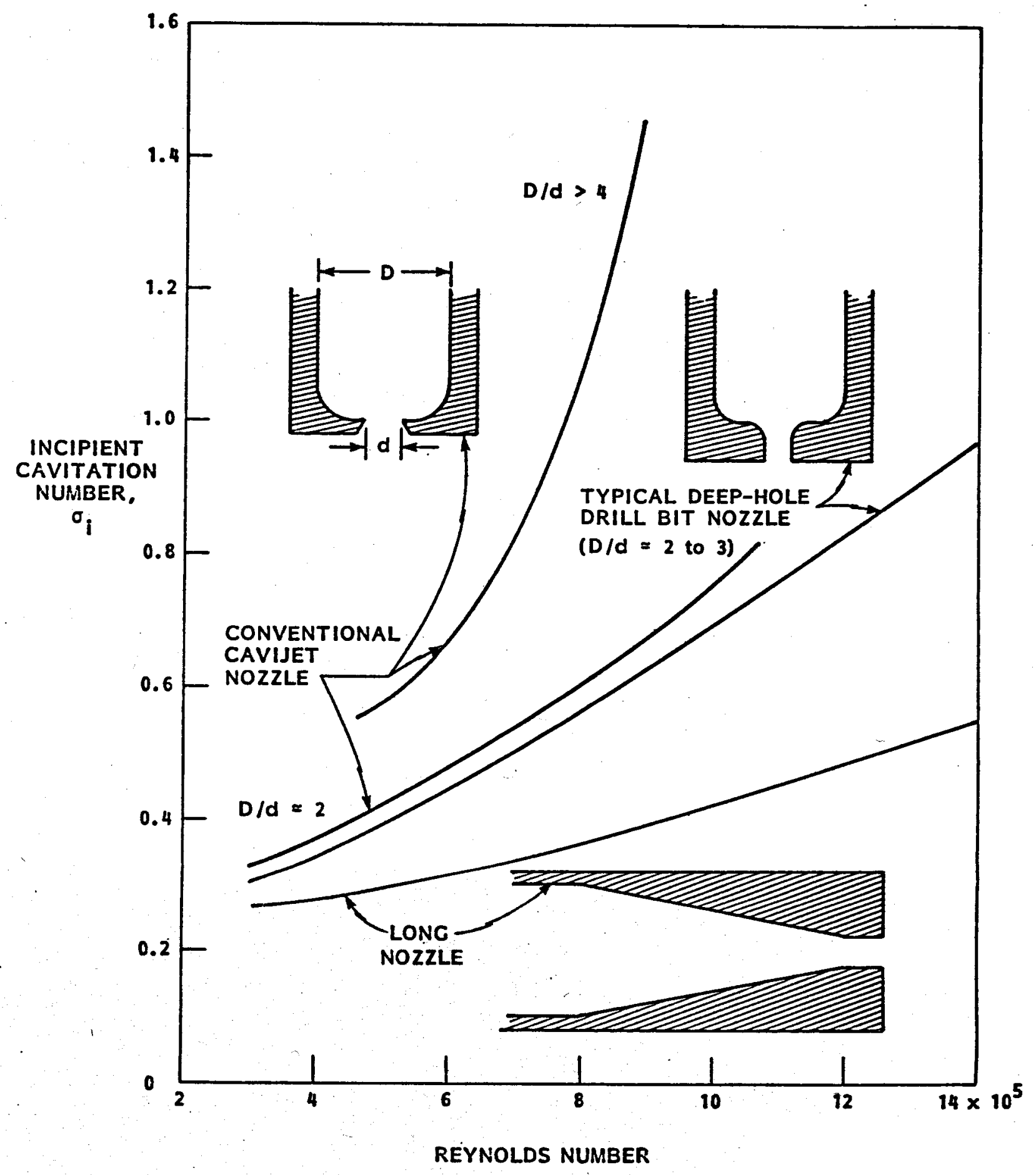

FICURE 1 - APPROXIMATE INCIPIENT CAVITATION NUMBERS FOR CAVIJET ${ }^{\circledR}$ AND OTIAER NOZZLE TYPES (ACOUSTIC DETECTION) 
The data presented in Figure 1 are particularly important because they are a bench mark against which new nozzles may be compared. New nozzles with smaller contraction ratios, e.g., $D / d \simeq 2$, were sought which would have higher incipient cavitation numbers than typical deep-hole drill bit nozzles and also equal or exceed the $\sigma_{i}$ of the basic CAVIJET ( $D / d \geq 4$ ). The following sections present and discuss the incipient cavitation data obtained on some new nozzle concepts. The measured incipient cavitation numbers for these new nozzle systems are reported relative to the measured cavitation number for the basic $D / d=4$ CAVIJET so as to indicate the existence of improvements and the magnitude of such improvements.

\subsubsection{CAVIJET nozzles with central devices - As reported} in References 6 to 9, CAVIJET applications in air or submerged at near atmospheric ambient pressures generally incorporate central devices (center bodies or vortex generators) to cause cavitation in the center of the jet. Such center jet cavitation is known to be more damaging because the cavities formed are collapsed nearer to the stagnation point of the jet. However, centerbodies are probably not practical in the deep hole drilling application. This is because of the reduction to the clear dimension at the nozzle exit (for passing solid material) which increases the probability of clogging the nozzle. The same disadvantage exists for central vortex devices if the vortex generator is located in the nozzle exit (where it is most effective). However, if the vanes (vortex generators) could be located in the larger diameter supply pipe they might be practical, and some large scale experiments were carried out to examine the feasibility of vortex systems. As pointed out in Section 2.2.2, where these tests are described, central vortex systems can generate central cavities with $\sigma_{i} \geq 3$, but such high 
cavitation numbers required the vanes to be located in the nozzle exit or throat.

Figure 2 shows incipient cavitation data for a basic CAVIJET having a blunt-based,circular-cylinder center body with diameter half that of the nozzle exit diameter. For this center body nozzle, cavitation was observed to occur almost simultaneously in the shear zone on the periphery of the jet and in the wake of the center body. The incipient cavitation number was equal to or slightly higher than for the basic CAVIJET nozzle without center body; that is, $\sigma_{i} / \sigma_{i, 0} \simeq 1.1$.

Figure 2 also shows the results obtained with a basic CAVIJET nozzle having a contraction ratio of two (obtained by

a $68.8 \mathrm{~mm}$ ( 2.71 in.) long insert in the $33.5 \mathrm{~mm}$ (1.32 in.) diameter supply pipe) in which a pair of $9.4 \mathrm{~mm}$ (0.37 in.) chord, opposed foils (vortex generators) was installed. As shown in Figure 2, remarkably high values of $\sigma_{i} / \sigma_{i, 0}$ were observed for this configuration. Figure 3 is a photograph of developed cavitation for the nozzle with vane tested. Note that cavitation is occurring in periodic bursts at the periphery of the jet and, even at the low test cavitation number of 0.25 , no cavitation is observed at the center of the jet. The high values of $\sigma_{i}$ obtained, the periodic structured cavities in the periphery of the jet, and the absence of central vortex cavitation were clearly resulting from the organ-pipe configuration that had been formed by the nozzle insert used for this test. This deduction was confirmed when the vanes were removed and essentially no change took place in the observed cavitation indices. These test results, when later compared with the other nozzles tested in the preliminary screening, motivated a future concentration of the program on obtaining a better understanding of the ORGANPIPE CAVIJET system and on the development of STRATOJET nozzle 


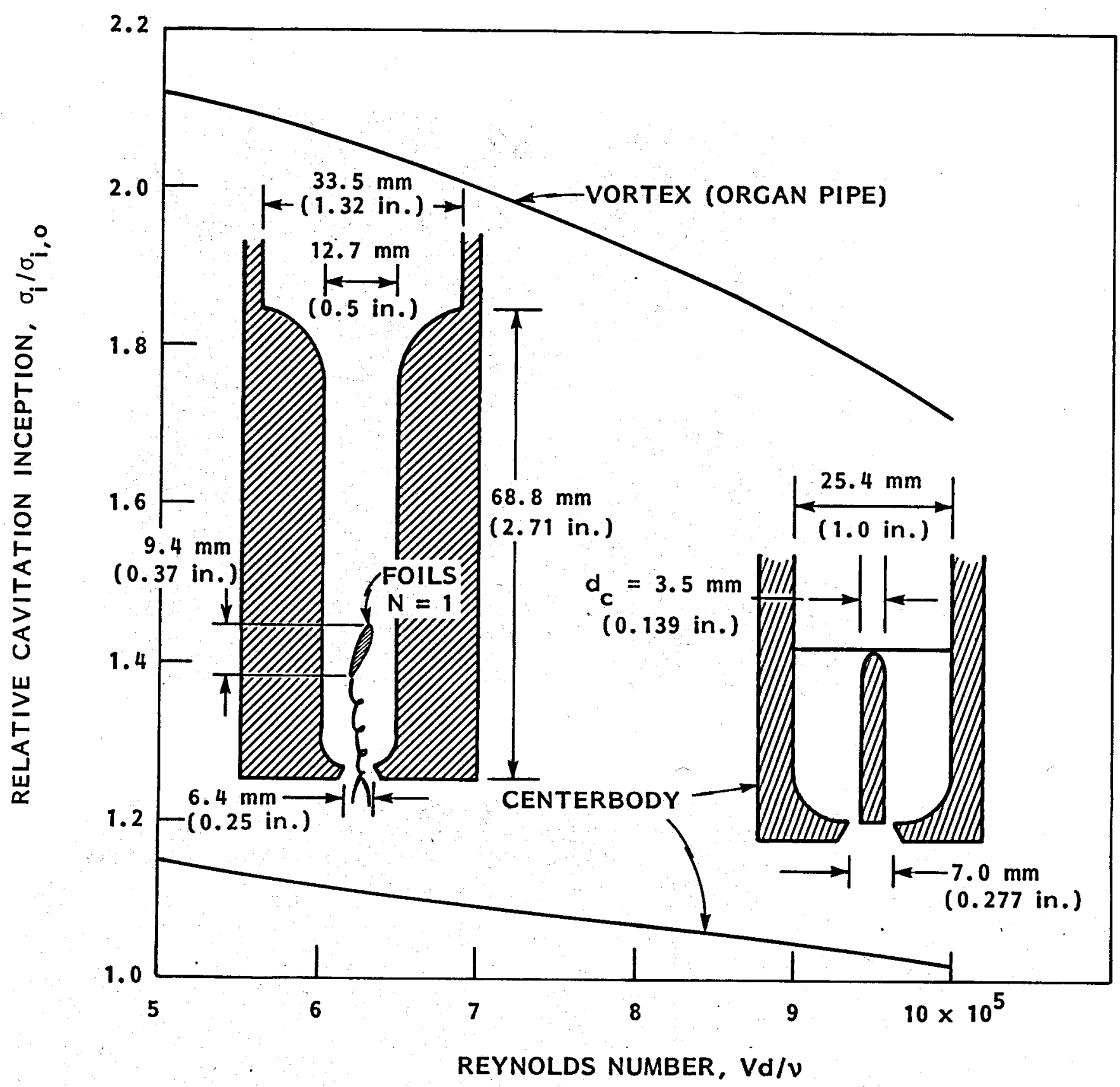

FIGURE 2 - EXPERIMENTAL INCIPIENT CAVITATION NUMBERS FOR VORTEX AND CENTERBODY CAVIJET ${ }^{\circledR}$ NOZZLES; $\sigma_{i, 0}$ is incipient cavitation number for plain CAVIJET nozzle 


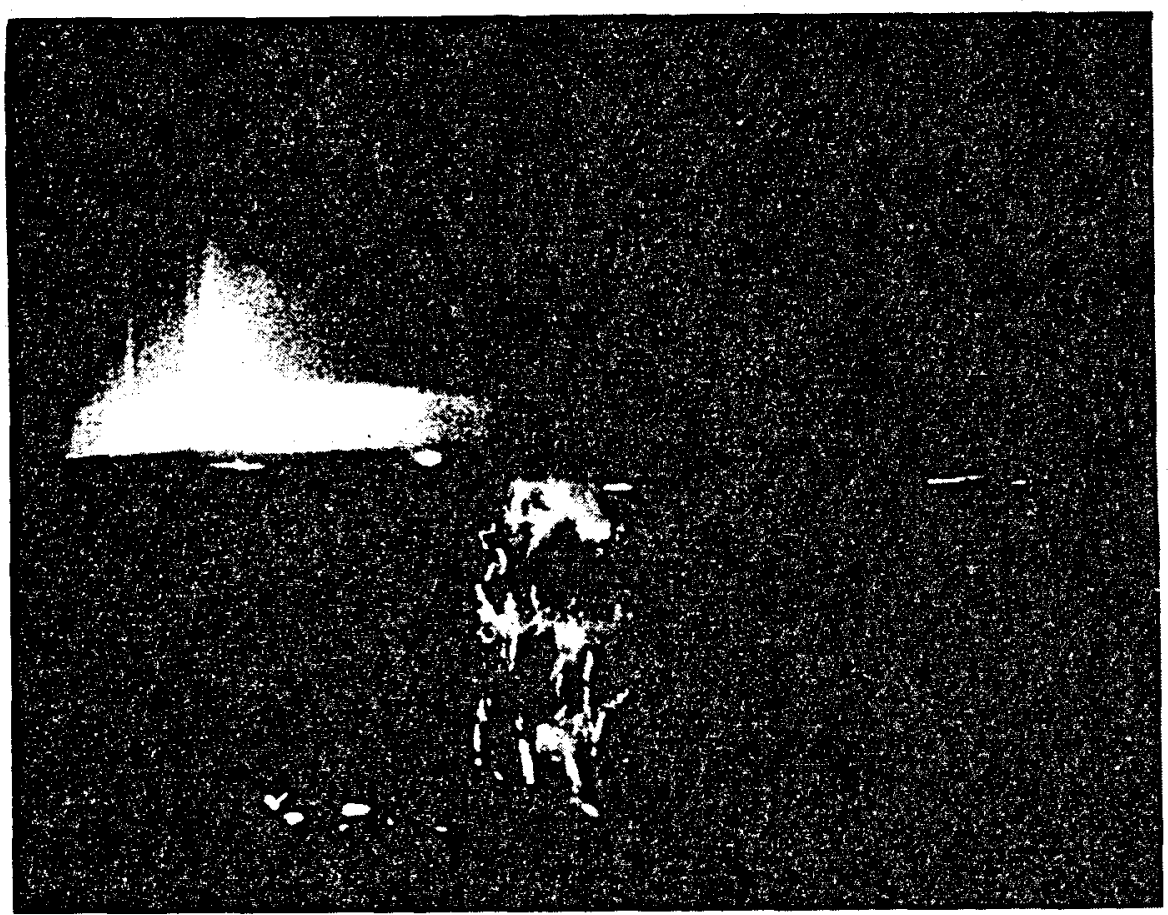

FIGURE 3 - CAVITATION PATTERN FOR VORTEX (ORGAN-PIPE) CAVIJET ${ }^{\circledR}$ NOZZLE; $\sigma=0.25, d=6.4 \mathrm{~mm}(0.25 \mathrm{in.}$ ), $D=12.7 \mathrm{~mm}(0.50 \mathrm{in.}), X=22.3 \mathrm{~mm}(0.88 \mathrm{in}$.$) (see$ Figure 2) 
systems for the drill bit application.

2.1.3 PULSER CAVIJET nozzle system - In Reference 2, it was shown that several types of passive hydro-acoustic nozzle systems for causing structuring of the jet into discrete ring vortices were feasible. Considerable promise was assigned to the PULSER-CAVIJET because of the remarkably high values of jet velocity oscillations that were measured in air. However, it was recognized that in liquids, cavitation would probably occur first in the PULSER chamber and further that the discrete rings formed in the chamber may be disrupted by the downstream orifice so that well-defined vortex rings would not occur in the outer flow.

Figure 4 shows cavitation inception data for the typical PULSER CAVIJET nozzle system tested. Cavitation was first observed at the downstream nozzle exit at values of $\sigma_{1} / \sigma_{i, 0} \simeq 1.5$. It was not possible to see inside the metal chamber where it is believed that cavitation occurred at much higher cavitation numbers, On the impact surface the ratio of $\sigma_{i} / \sigma_{i, 0}$ was about 1.0. At the impact surface, cavitation randomly occurred - discrete ring vortices were not observed, Thus, PULSER CAVIJET nozzles did not show improved cavitation characteristics on the impact surface - presumably for the reasons discussed in the previous paragraph.

2.1 .4 ORGAN-PIPE CAVIJET nozzle system - Figure 5 presents cavitation inception data for the ORGAN-PIPE CAVIJET shown. The exit nozzle was a conventional CAVIJET with a diameter contraction ratio, $D / d=3.5$. No attention was paid to the details of the throat length and exit contour which were about $0.8 \mathrm{~mm}$ (1/32 in.) and a $45^{\circ}$ chamfer respectively. As described in Appendices $A$ and $C$, these details were found to greatly 


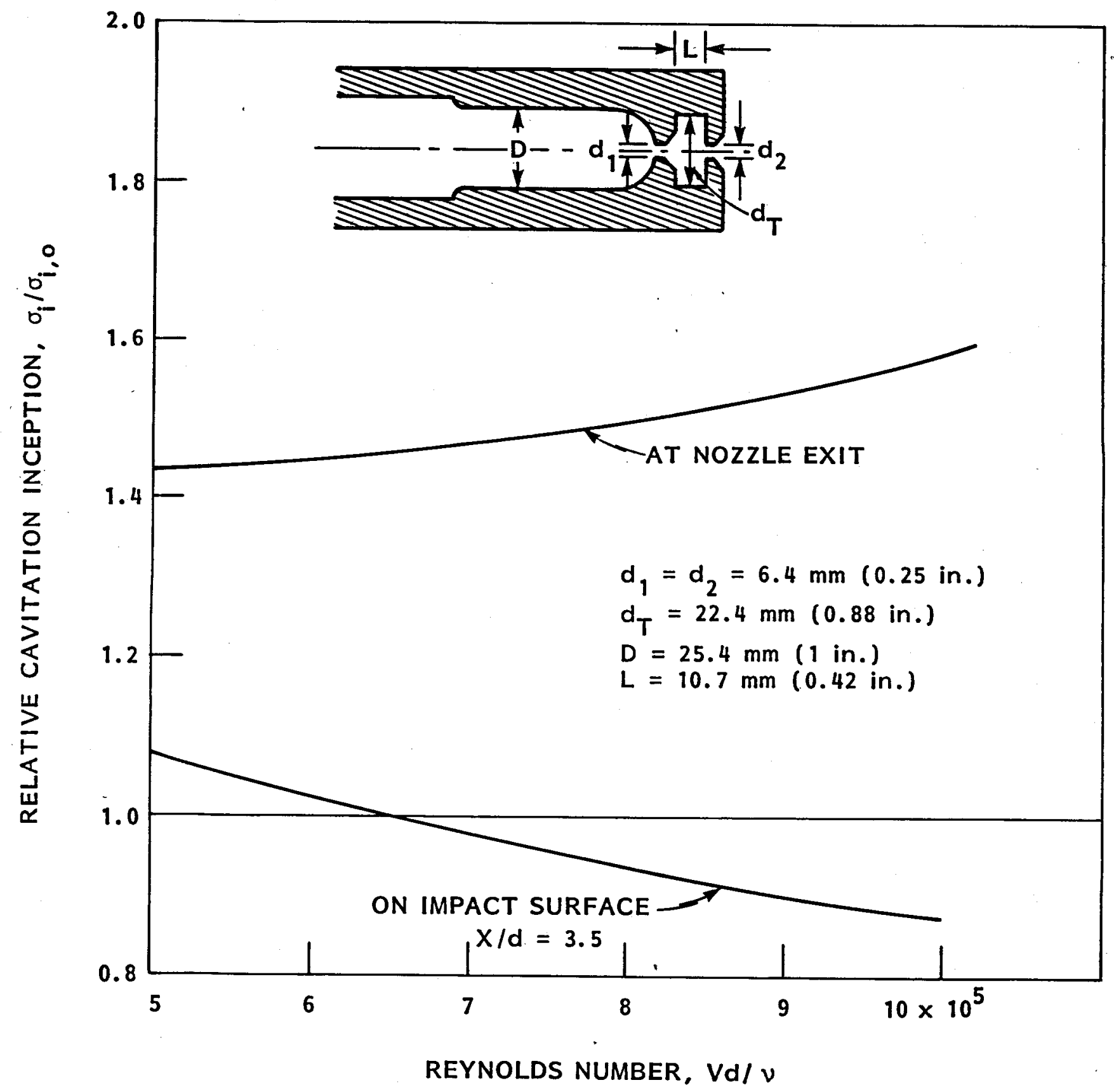

FIGURE 4 - EXPERIMENTAL INCIPIENT CAVITATION NUMBERS FOR PULSER CAVIJET $\odot$ NOZZLE; $\sigma_{i, 0}$ is incipient cavitation number for plain CAVIJET nozzle 


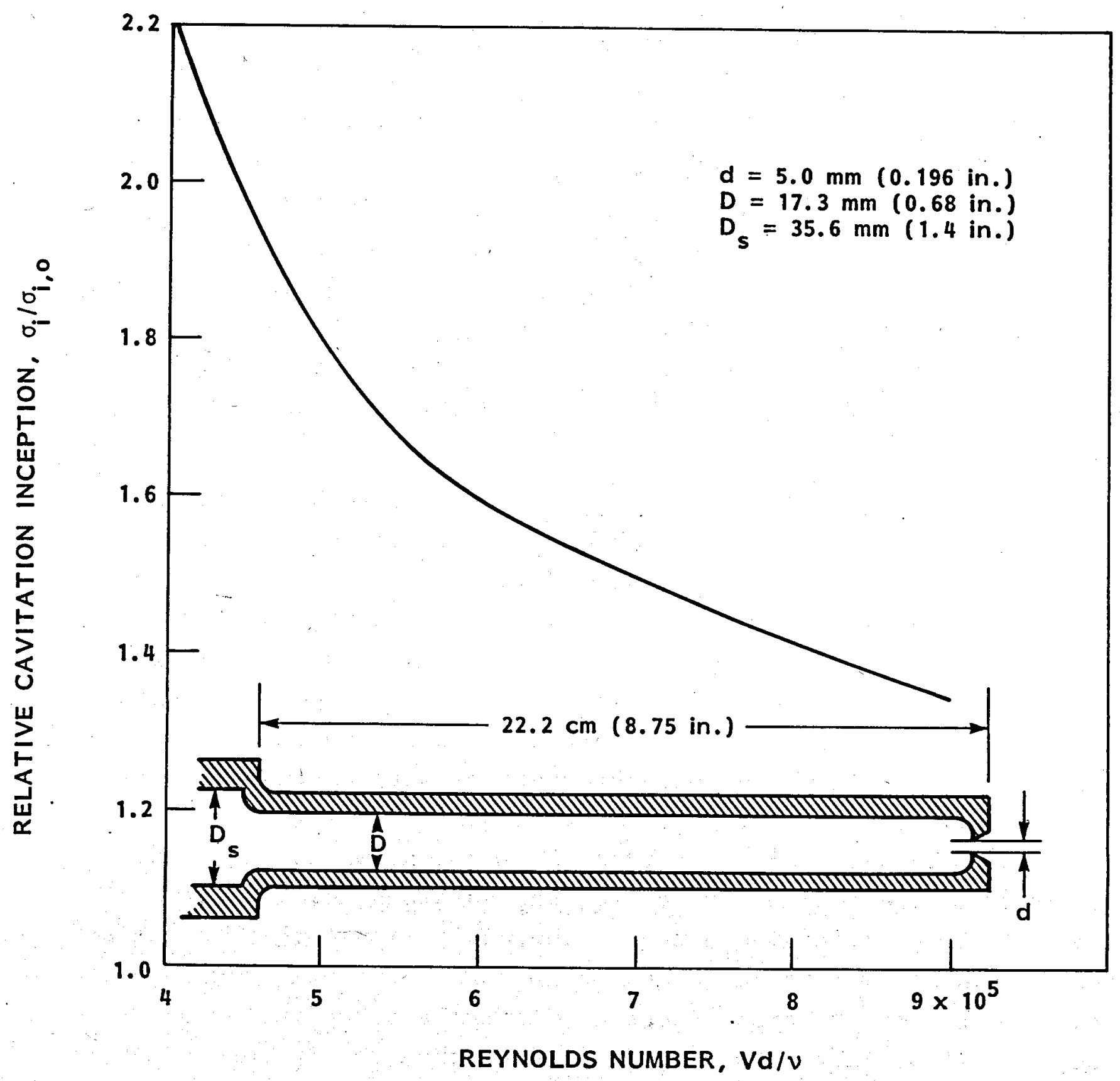

FIGURE 5 - EXPERIMENTAL INCIPIENT CAVITATION NUMBERS FOR ORGAN-PIPE CAVIJET NOZZLE; $\sigma_{i, 0}$ is incipient cavitation number for plain CAVIJET nozzle 
influence both the Strouhal number and the amplitude of oscillation of such an ORGAN-PIPE nozzle system. The very large values of $\sigma_{i} / \sigma_{i, 0}$ at a Reynolds number of $4 \times 10^{5}$ indicated that this nozzle was resonating, with peak performance at a velocity lower than the test velocity. The most important point to note is the very large value of $\sigma_{i} / \sigma_{i, 0}>2.2$ for a nozzle system having a contraction ratio of 3.5 . Again, the ORGAN-PIPE concept was shown to be a very promising candidate for futher development.

\subsubsection{PULSER-FED CAVIJET nozzle system - In Reference 2,} it was pointed out that the problems related to internal chamber cavitation of PULSER CAVIJET nozzles(also described in the previous paragraphs)might be alleviated in the PULSER-FED CAVIJET configuration. Figure 6 shows such a configuration and its incipient cavitation characteristics. Although the photographic technique available at the time of these tests precluded an acceptable photograph, visual observations with a strobe light clearly showed cavitation occurring in discrete ring vortices. The peak value of $\sigma_{i} / \sigma_{i, 0} \simeq 2.2$ at $R_{e} \simeq 7.5 \times 10^{6}$ confirms that structuring the jet into discrete ring vortices does increase the incipient cavitation number. The cavitation number for this PULSER-FED CAVIJET is based on the $\triangle p$ across the exit nozzle. The actual pressure drop across the entire system (including the PULSER) was about 20 percent greater than the $\Delta p$ across the nozzle and it is this energy loss that detracts from the PULSER-FED CAVIJET as a candidate for further development in deep hole drill-bits.

\subsubsection{Summary and conclusions of preliminary inception}

tests - Figure 7 compares all of the nozzle systems tested. In view of the high values of $\sigma_{i} / \sigma_{i, 0}$ achieved with the relatively simple ORGAN-PIPE CAVIJET configuration, and the energy loss 


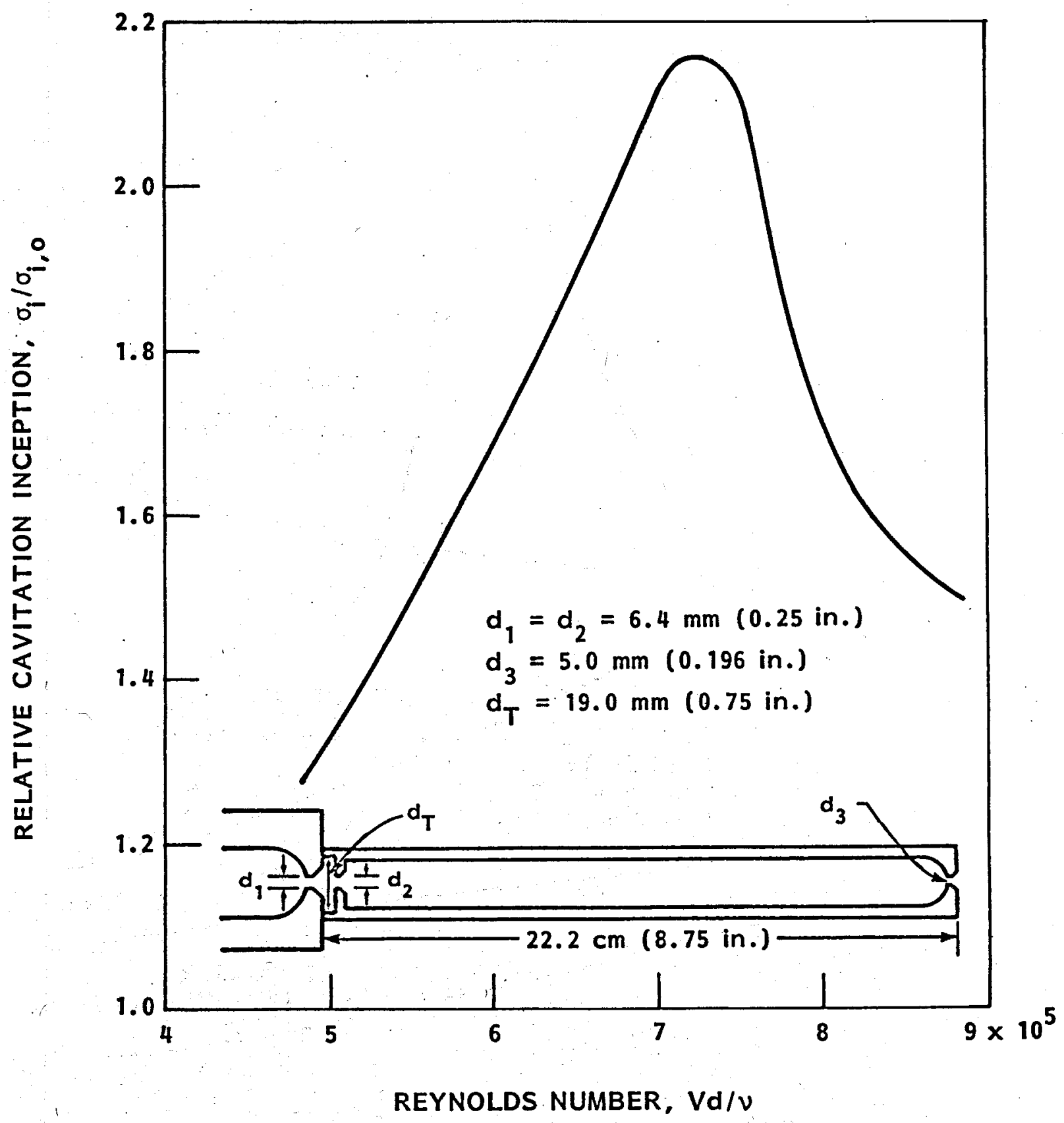

FIGURE 6 - EXPERIMENTAL INCIPIENT CAVITATION NUMBERS FOR PULSER-FED CAVIJET ${ }^{\circledR}$ NOZZLE $; \sigma_{i, 0}$ is incipient cavitation nurber for plain CAVIJET nozzle 


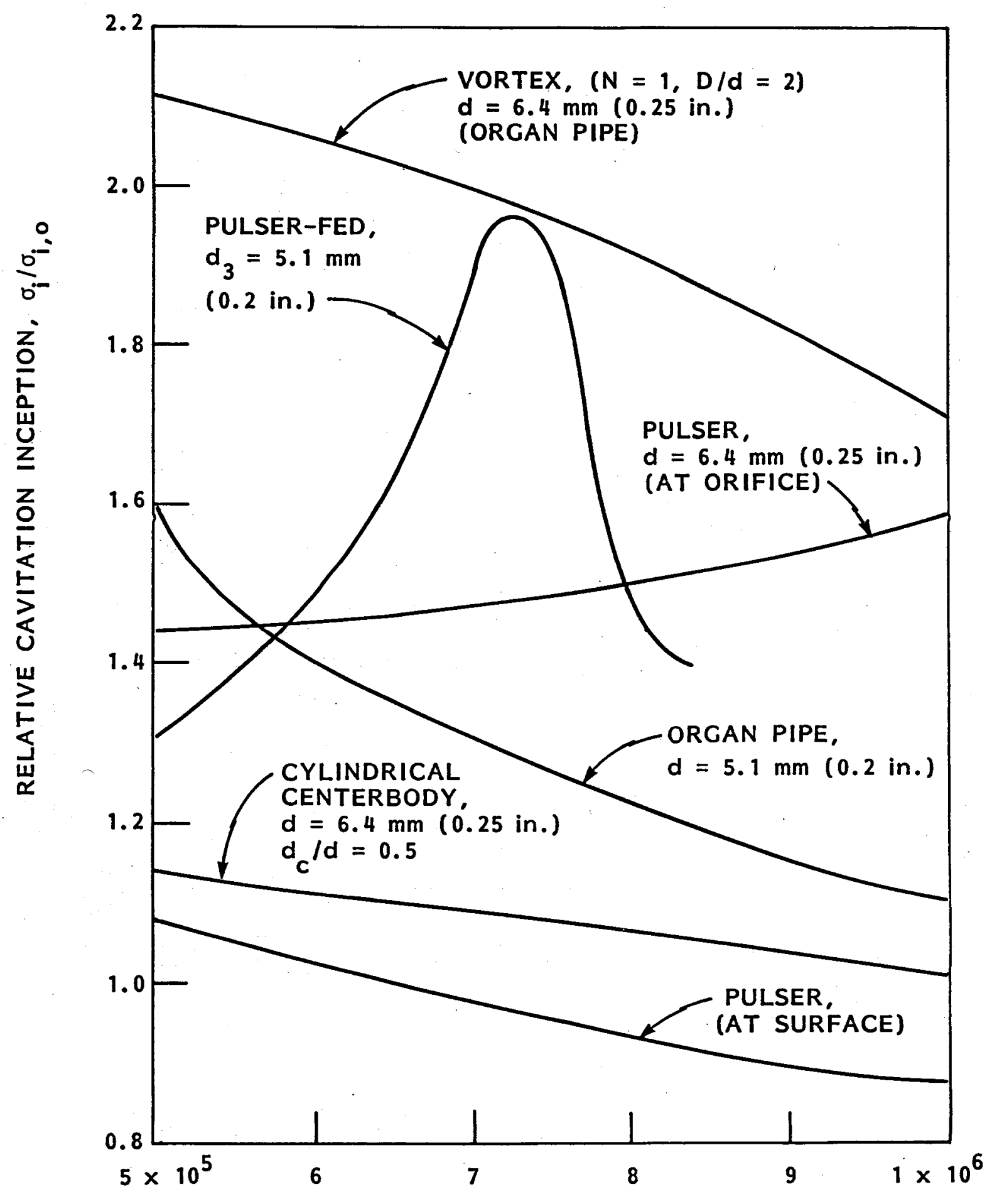

REYNOLDS NUMBER, Vd/V

FIGURE 7 - EXPERIMENTAL INCIPIENT CAVITATION NUMBERS FOR VARIOUS CAVIJET ${ }^{\circledR}$ NOZZLE TYPES; $\sigma_{i, 0}$ is incipient cavitation number for plain CAVIJET nozzle 
associated with the more complex PULSER-FED configuration, it was concluded that the ORGAN-PIPE CAVIJET should be selected for extensive further development. In particular, design procedures were required to optimize the geometry of the organ pipe for resonance at a required pressure drop, and to optimize the details of the nozzle geometry so that its feed-back characteristics were also matched to the required pressure drop. Discussions of these optimization studies can be found in Appendices $A$ and $C$. The PULSER, PULSER-FED and ORGAN-PIPE CAVIJETS all depend on the same hydro-acoustic phenomenon to achieve a self-excited resonance. An acronym was coined to refer to these systems STRuctured Acoustically Tuned Oscillating JET, "STRATOJET". Henceforth such nozzle systems, and particularly the ORGAN-PIPE CAVIJET, are called STRATOJETS.

\subsection{Vortex Generating Nozzles}

2.2.1 Theory - Let us consider a nozzle of upstream area, $A_{u}$, and downstream area, $A_{d}$ (see Figure 8 ). The area contraction ratio, $K_{C}$, is defined as:

$$
K_{c} \equiv A_{u} / A_{d}
$$

A linear vortex of strength, $\Gamma$, is generated on the tube axis, and we seek the pressure variation along this axis. $v_{u}$ is the assumed uniform axial velocity at the upstream section and $v_{d}$ is the assumed uniform axial velocity at the downstream section outside the vortical viscous core of the vortex. We denote $p_{u}(r), v_{u}(r), w_{u}(r)$ and $p_{d}(r), v_{d}(r), w_{d}(r)$ as the pressures, axial velocities and tangential velocities in the upstream and downstream sections at a distance, $r$, from the vortex axis (Figure 8). The total dynamical pressure, $p_{0}$, is imposed in the feed line and $\mathrm{p}_{a}$ is the ambient pressure where the jet is 

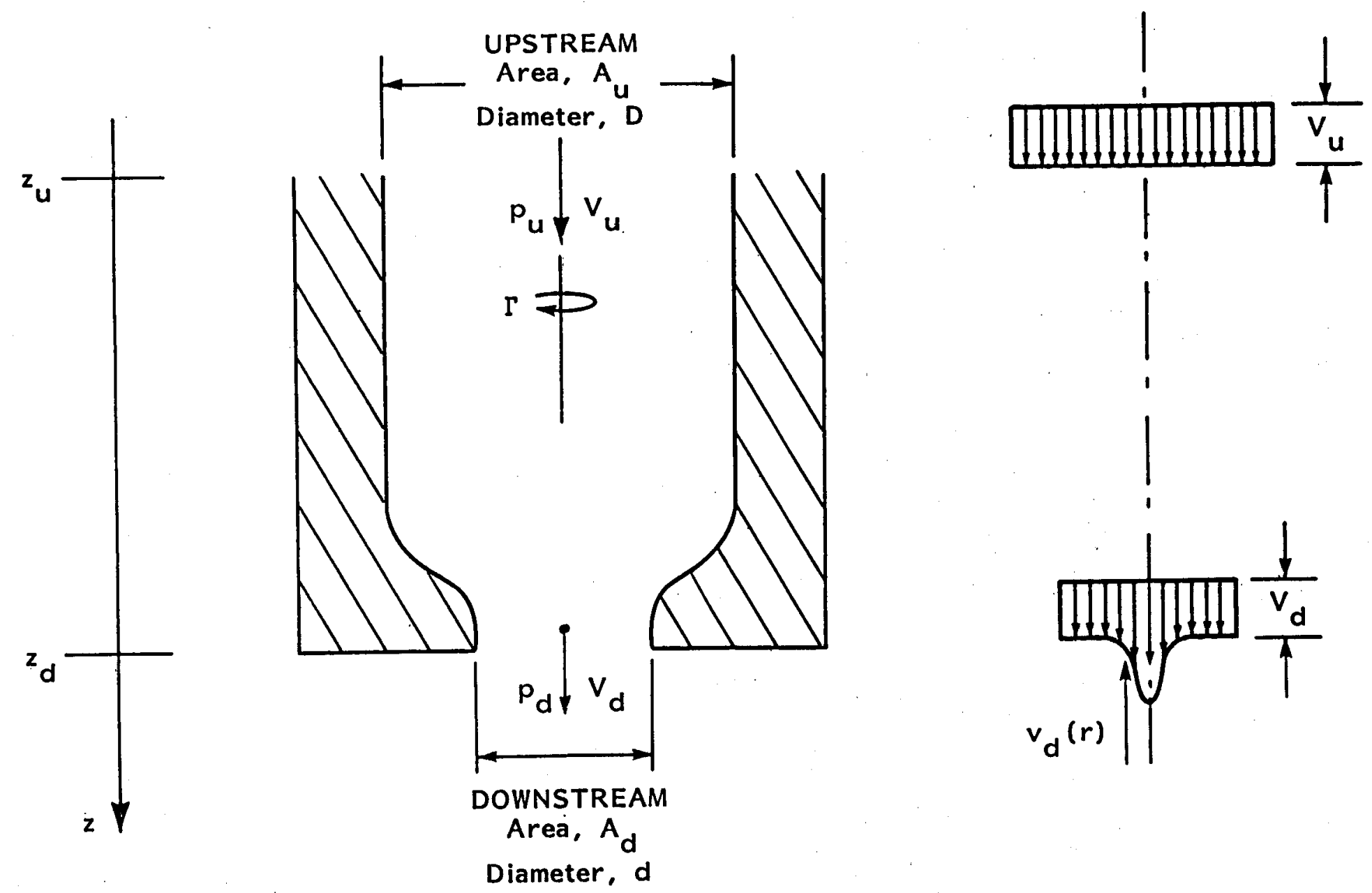

FIGURE 8 - PARAMETERS FOR VORTEX NOZZLE ANALYSIS 
discharged.

To address the problem in its generality one has to solve the Navier-Stokes equations. In the tube areas where the radial velocities are negligible the axisymnetry of the problem simplifies the continuity equation to:

$$
\partial \mathrm{v} / \mathrm{d} z=0,
$$

and the Navier-Stokes equations are:

$$
\begin{gathered}
\frac{w^{2}}{r}=\frac{1}{\rho} \frac{\partial p}{\partial r}, \\
v \frac{\partial w}{\partial z}=v\left\{\frac{\partial}{\partial r}\left[\frac{1}{r} \frac{\partial(r w)}{\partial r}\right]+\frac{\partial^{2} w}{\partial z^{2}}\right\}, \\
0=-\frac{1}{\rho} \frac{\partial p}{\partial z}+v \frac{1}{r} \frac{\partial}{\partial r} \quad\left(r \frac{\partial v}{\partial r}\right)
\end{gathered}
$$

Unfortunately only a numerical solution of this system is possible. Another approach consists in neglecting the direct effects of viscosity, and to account for it by considering the flow to be rotational in the vortex core. Batchelor (10) considered the problem with such an assumption using the stream function equation. He assumed an upstream uniform axial velocity, $v_{u}$, with a rigid body angular velocity, $\Omega_{u}$, in the core and derived the flow field downstreám. We can sumarize his results and extend them for our purpose as follows.

If the core radius has the values: $a_{u}$, upstream, and $a_{d}$, downstream, then the velocities in the rotational tube are as follows (see (10), p. 548): 


$$
\begin{aligned}
& \frac{v_{d}(r)}{v_{u}}=1+\left(\frac{a u^{2}}{a_{d}^{2}}-1\right) \frac{\alpha a_{d} J_{o}(\alpha r)}{2 J_{1}\left(\alpha a_{d}\right)} ; r<a_{d} \\
& \frac{w_{d}(r)}{\Omega r}=1+\left(\frac{a_{u}^{2}}{a_{d}^{2}}-1\right) \frac{a_{d} J_{1}(\alpha r)}{r J_{1}\left(\alpha a_{d}\right)} ; r<a_{d}
\end{aligned}
$$

where we define: $\alpha \equiv 2 \Omega / \mathrm{V}_{\mathrm{u}}$, and $\mathrm{J}_{0}$ and $\mathrm{J}_{1}$ are the Bessel functions of the first kind respectively, of order zero and one. The upstream conditions are (Figures 8, 9):

$$
\left.\begin{array}{l}
\mathrm{v}_{\mathrm{u}}=\text { constant } ; \\
\mathrm{w}_{\mathrm{u}}(\mathrm{r})=\Omega \mathrm{r} ;
\end{array}\right\} \mathrm{r} \leq \mathrm{a}_{\mathrm{u}}
$$

Outside the viscous core we can assume that the flow is potential and such that:

$$
\begin{aligned}
& \left.\begin{array}{l}
v_{u}=\text { constant } ; \\
w_{u}(r)=\Omega a_{u}^{2} / r ;
\end{array}\right\} r \geq a_{u} \\
& \left.\begin{array}{l}
v_{d}=\text { constant } ; \\
w_{d}(r)=\Omega a_{d}^{2} / r ;
\end{array}\right\} r \geq a_{d}
\end{aligned}
$$

Mass conservation allows determination of a relationship between $v_{d}$ and $v_{u}$. When the vortical section is not negligible in comparison with $A_{d}$, this relation depends on the unknown core radius, $a_{d}$, 


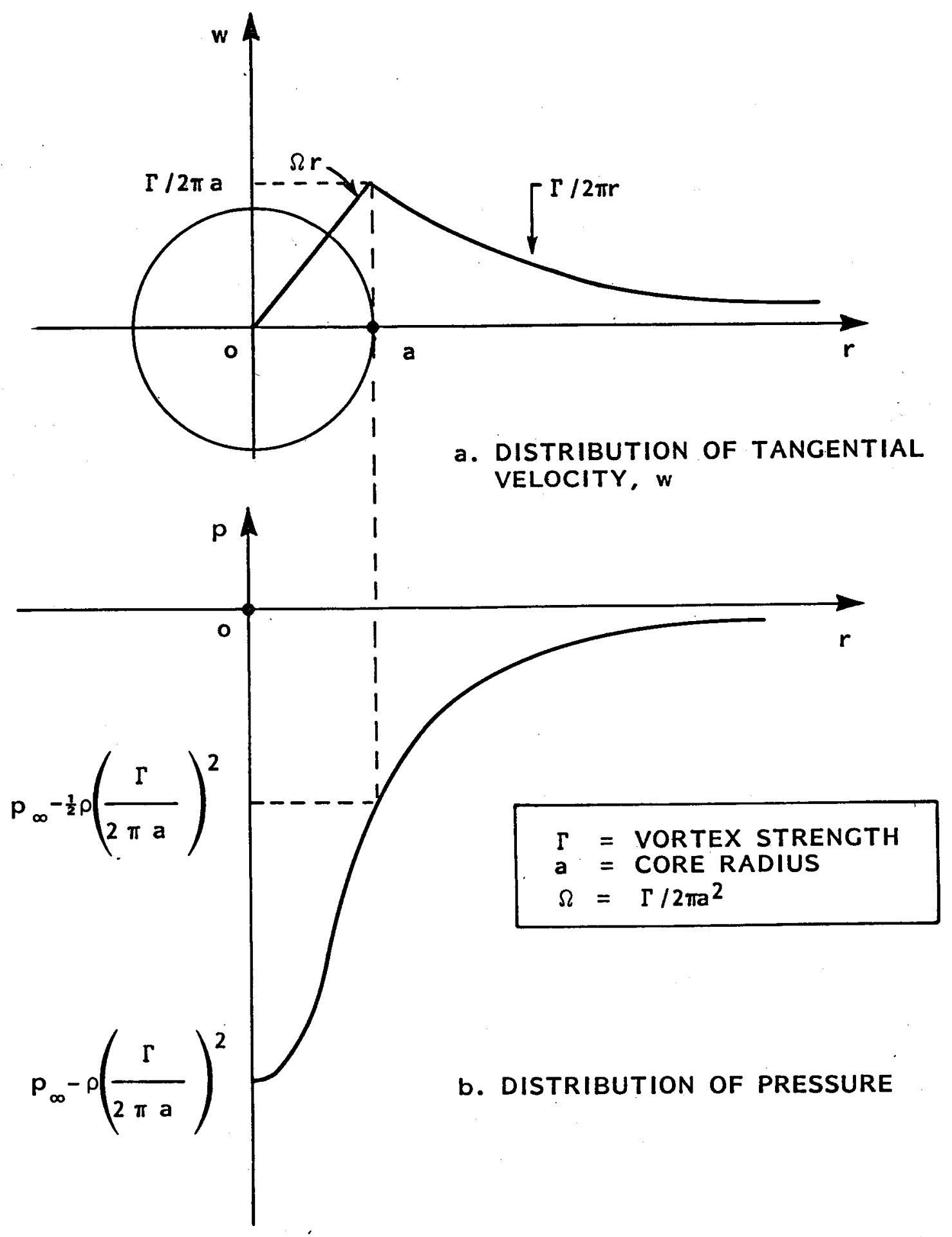

FIGURE 9 - DISTRIBUTION OF TANGENTIAL VELOCITY AND PRESSURE ASSOCIATED WITH A VORTEX 


$$
v_{u} A_{u}=v_{d}\left(A_{d}-\pi a_{d}^{2}\right)+2 \pi \int_{0}^{a} d v_{d}(r) r d r
$$

However, in the most practical cases where: $\pi a_{d}^{2}<4 A_{d}$, Equation [11] simplified considerably to give:

$$
v_{u}=v_{d} A_{d} / A_{u}=K_{c}^{-1} \cdot v_{d}
$$

Then, Equation [6], written at the core boundary, $r=a_{d}$, can be used to determine $a_{d}$ :

$$
K_{c}=I+\left(\frac{a_{u}^{2}}{a_{d}^{2}}-1\right) \frac{\alpha a_{d} J_{o}\left(\alpha a_{d}\right)}{2 J_{1}\left(\alpha a_{d}\right)}
$$

Once $a_{d}$ is known, Equations [6] and [7] can be used again to determine the flow field in the viscous core.

The pressure in the inviscid region of the flow can be determined with the Bernoulli equation. We can write on the outer edge of the viscous core tube:

$p_{0}=p_{u}\left(a_{u}\right)+\frac{1}{2} p\left(V_{u}^{2}+\Omega^{2} a_{u}^{2}\right)=p_{d}\left(a_{d}\right)+\frac{1}{2} \rho\left(V_{d}^{2}+\Omega^{2} a z_{d}\right)$

When $\pi a_{d}^{2} \ll<A_{d}$, Equation [12] applies and we have at the core boundary:

$$
\begin{gathered}
p_{d}\left(a_{d}\right)=p_{0}-\frac{2}{2} \rho\left(K_{c}^{2} V_{u}^{2}+\Omega^{2} a z\right) \\
p_{u}\left(a_{u}\right)=p_{0}-\frac{3}{2} \rho\left(V_{u}^{2}+\Omega^{2} a_{u}^{2}\right) .
\end{gathered}
$$


To determine the pressure at the center of the vortex, Equation [3] should be integrated using the expressions [7] for $w_{d}(r)$, and $[8]$ for $w_{u}(r)$.

$$
\begin{array}{r}
p_{u}\left(a_{u}\right)-p_{u}(0)=\rho \int_{0}^{a} u \Omega^{2} r d r, \quad[17] \\
p_{d}\left(a_{d}\right)-p_{d}(0)=0 \int_{0}^{a} d \Omega^{2} r\left[1+\left(\frac{a_{u}^{2}}{a_{d}{ }^{2}}-1\right) \frac{a_{d}}{r} \frac{J_{1}(\alpha r)}{J_{1}\left(\alpha a_{d}\right)}\right] d r \cdot[18]
\end{array}
$$

Combining [15] and [18], [16] and [17] we obtain:

$$
\begin{gathered}
p_{0}-p_{u}(0)=\frac{1}{2} \rho\left(V_{u}^{2}+2 \Omega^{2} a_{u}^{2}\right), \\
p_{0}-p_{d}(0)=\frac{1}{2} \rho\left[K_{c}^{2} V_{u}^{2}+2 \Omega^{2} a_{d}^{2}+2 \Omega^{2} a_{d}^{2}\left(\frac{a_{u}^{2}}{a_{d}^{2}}-1\right) \frac{1-J_{0}\left(\alpha a_{d}\right)}{\alpha J_{1}\left(\alpha a_{d}\right)}[20]\right.
\end{gathered}
$$

Cavitation will appear in the vortical core as soon as $p_{d}(0)$ drops below a critical pressure, let us say for simplification the vapor pressure. To enhance cavitation it is hence necessary, for a given ambient pressure, $p_{a}$, and pump pressure, $p_{0}$ (thus, jet velocity, $v_{d}$ ) to reduce the value of $p_{d}(0)$ to a minimum. This is attained if the cavitation number, $\sigma$, is maximized;

$$
\sigma=-\min \left(c_{p_{d}}\right)=\frac{p_{a}-p_{d}(0)}{\frac{2}{2} \rho V_{d}^{2}}
$$

where

$$
P_{a}=P_{o}-\frac{1}{2} \rho V_{d}^{2}
$$


Equation [22] assumes that the jet diameter, and thus the distance between the location at which $\mathrm{p}_{\mathrm{a}}$ is defined and the jet axis, is big enough compared to the vortical core radius to allow neglect of the pressure drop due to the rotation. Equations [20] and [21] and [13] combined give:

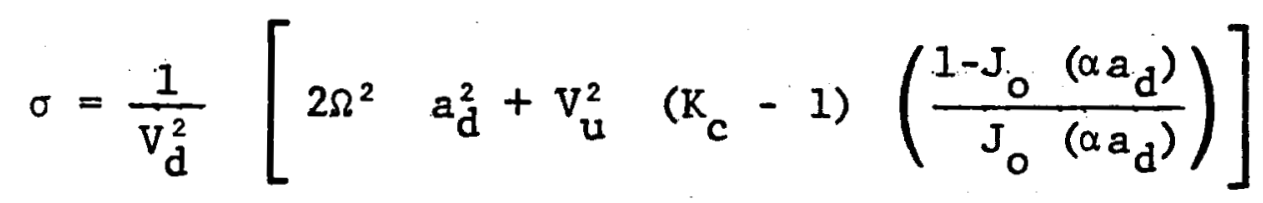

This can also be written as:

$$
\sigma=\frac{3}{2} \beta^{2}+\frac{K_{c}-1}{K_{c}^{2}} \cdot \frac{1-J_{0}\left(\beta K_{c}\right)}{J_{0}\left(\beta K_{c}\right)} \text {. }
$$

where we define:

$$
B=2 \Omega \quad a_{d} / V_{d}
$$

Now, we can discuss the optimum value of $K_{c}$, by considering Equations [24] and [13]

When at the nozzle exit the tangential velocity at the core boundary, $\Omega a_{d}$, is small compared to the jet exit velocity, $v_{d}$. $\beta \ll 1$, then conclusions on $\sigma$ and $k_{c}$ can be drawn easily by expanding $J_{0}$ and $J_{\perp}$ in powers of $\beta$ in [24] and [13], One obtains:

$$
\begin{aligned}
& a_{u^{\prime}} / a_{d}=\sqrt{K_{c}} \\
& \sigma \simeq\left(K_{c}+\frac{2}{4}\right) \beta^{2}=\left(K_{c}+\frac{1}{4}\right) \alpha^{2} a_{u}^{2} K_{c}^{-3},
\end{aligned}
$$

Let us emphasize that $\sigma$ and $a_{u}$ are functions only of the upstream conditions. 
Now if we use the fact that the vortex is produced by a wing of span, $b$, chord, $l$, and lift coefficient, $C_{L}$, then:

$$
\Omega=\frac{C_{L} \cdot \ell \cdot v_{u}}{2 \pi a_{u}^{2}}
$$

The hardest term to evaluate is the core radius, $a_{u}$. We consider here the two commonly used models in the literature.

- In the first model: (a) the core radius is directly proportional to the wing span (11),

$$
a_{u} \simeq 0.08 b
$$

- In the second model: (b) a depends on the Reynolds number based on the location, $z$, downstream from the wing (12),

$$
a_{u} \simeq\left(5.04 \mu z / V_{u}\right)^{\frac{1}{2}}
$$

With model (a) the value of $\sigma$, based on [26], is

$$
\sigma \simeq 16 c_{L}\left(\frac{\ell^{2}}{b}\right) k_{c}^{-8}\left(k_{c}+\frac{1}{4}\right)
$$

and with model (b)

$$
\sigma=0.02\left(\frac{v_{d} l}{v}\right)\left(\frac{l}{z}\right) \quad C_{L} k_{c}^{-4}\left(R_{c}+\frac{l}{4}\right) .
$$

We see that, for both models, $\sigma$ increases when the contraction factor, $\mathrm{K}_{\mathrm{c}}$, is lowered.

As shown in Section 2.2.2, this conclusion was confirmed experimentally and seems to hold even for non-negligible values 
of $B$, since the second term in [24] also depends on $\mathrm{K}_{c}{ }^{-2}$. An interesting situation is that for which $\mathrm{J}_{0}\left(\beta K_{c}\right)$ is very small, in which case $\sigma$ is very large. The first zero of $J_{0}\left(\beta K_{c}\right)$ occurs at $\beta \mathrm{K}_{\mathrm{c}}=2.4$. If

$$
\mathrm{BK}_{\mathrm{c}}=2.4+\varepsilon \text {, }
$$

then

$$
\left[1-\mathrm{J}_{0}\left(\beta \mathrm{K}_{\mathrm{c}}\right)\right] / \mathrm{J}_{\mathrm{o}}\left(\beta \mathrm{K}_{\mathrm{c}}\right) \simeq 1.92 \varepsilon^{-1} \text {, }
$$

and from [13]:

$$
a_{d} \simeq\left(\frac{\varepsilon}{\sqrt{2\left(K_{c}-1\right)}}\right) a_{u} \text {. }
$$

The value of $\sigma$ in this case is given by:

$$
\sigma \simeq \frac{2 \Omega^{2} \mathrm{a}_{\mathrm{u}}{ }^{2}}{\mathrm{~V}_{\mathrm{d}}{ }^{2}} \frac{\varepsilon^{2}}{2 \mathrm{~K}_{\mathrm{c}}-1}+1.9 \frac{\mathrm{K}_{\mathrm{c}}-1}{\mathrm{~K}_{\mathrm{c}}{ }^{2}} \varepsilon^{-1}
$$

which for $\mathrm{K}_{c} \geq 1$ gives:

$$
\sigma \simeq 1.9 \frac{\mathrm{K}_{c}-1}{\mathrm{~K}_{c}^{2}} \varepsilon^{-1}
$$

This configuration can be very important for enhancing the cavitation inception if it can be produced practically. To do so one needs to satisfy [31], and thus have the following relation between the input variables: 


$$
\frac{2 \Omega a_{u}}{v_{u}}=\frac{2.4 \sqrt{2\left(k_{c}-1\right)}}{\varepsilon} k_{c}
$$

Equation [37] indicates that the input flow should have the highest possible ratio of tangential to normal velocities. Even then, as seen in Equations [30], [31] and [36], $\mathrm{K}_{c}$ must be kept to a minimum for the best results with this type of nozzle.

\subsubsection{Experimental studies - The HYDRONAUTICS High Speed} Channel (HSC) test section was modified to allow studies of large scale nozzles to be made. The use of large scale models was motivated, particularly for the case of internal vortex generators, to achieve detailed flow visualization and fine control of geometrical details.

The HSC is described in Reference 13. The test section is rectangular, $0.6 \mathrm{~m}$ wide by $3.7 \mathrm{~m}$ long ( $2 \mathrm{ft}$ by. $12 \mathrm{ft}$ ). The pump drive is a $743 \mathrm{~kW}$ ( $1000 \mathrm{hp}$ ) motor, with eddy-current coupling; rated at $2,200 \mathrm{l} / \mathrm{s}(35,000 \mathrm{gpm})$ at a $9.8-\mathrm{m}(32-\mathrm{ft})$ head. The depth of flow is normally controlled by means of an adjustable sluice gate just ahead of the test section. The system pressure can be varied from atmospheric conditions to a reduced pressure of less than $0.9 \mathrm{~m} \mathrm{(} 3 \mathrm{ft}$ ) of water absolute by means of a regulated vacuum pump system. The test section has clear acrylic windows on both sides and bottom for observation and photographic purposes.

The principal modification to the HSC was to replace the sluice gate with a baffle plate and axisymmetric contraction (bellmouth) as sketched in Figure 10. The flow then entered a $27.9 \mathrm{~cm}$ (II-in.) I.D. clear acrylic tube that fed the CAVIJET nozzle contraction supported by the bottom of the test section, as shown in the sketch. Two interchangeable nozzles were 


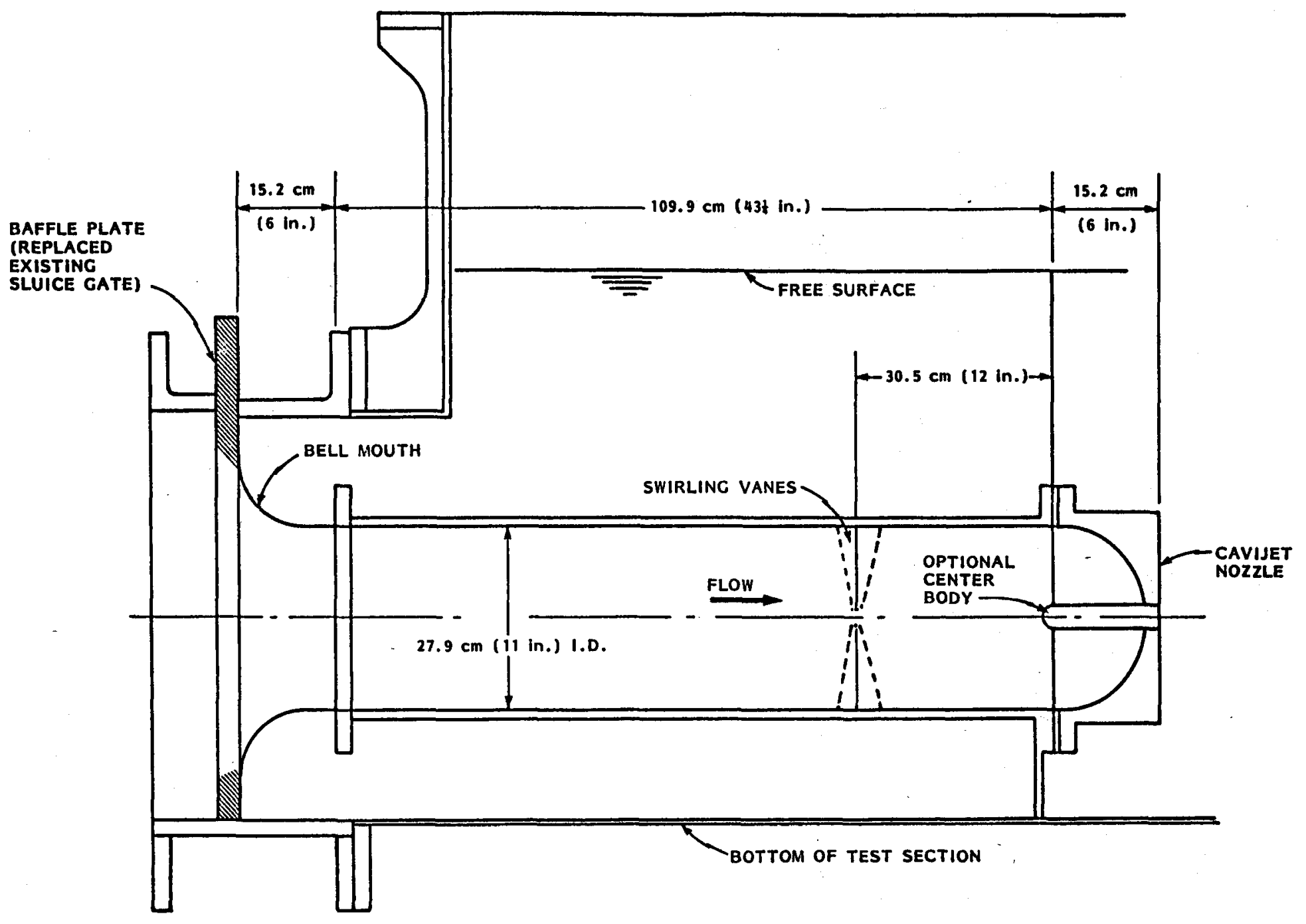

ficure 10 - LARGe scale nOzzLE INSTALLATION IN THE high SPEED CHANNEL 


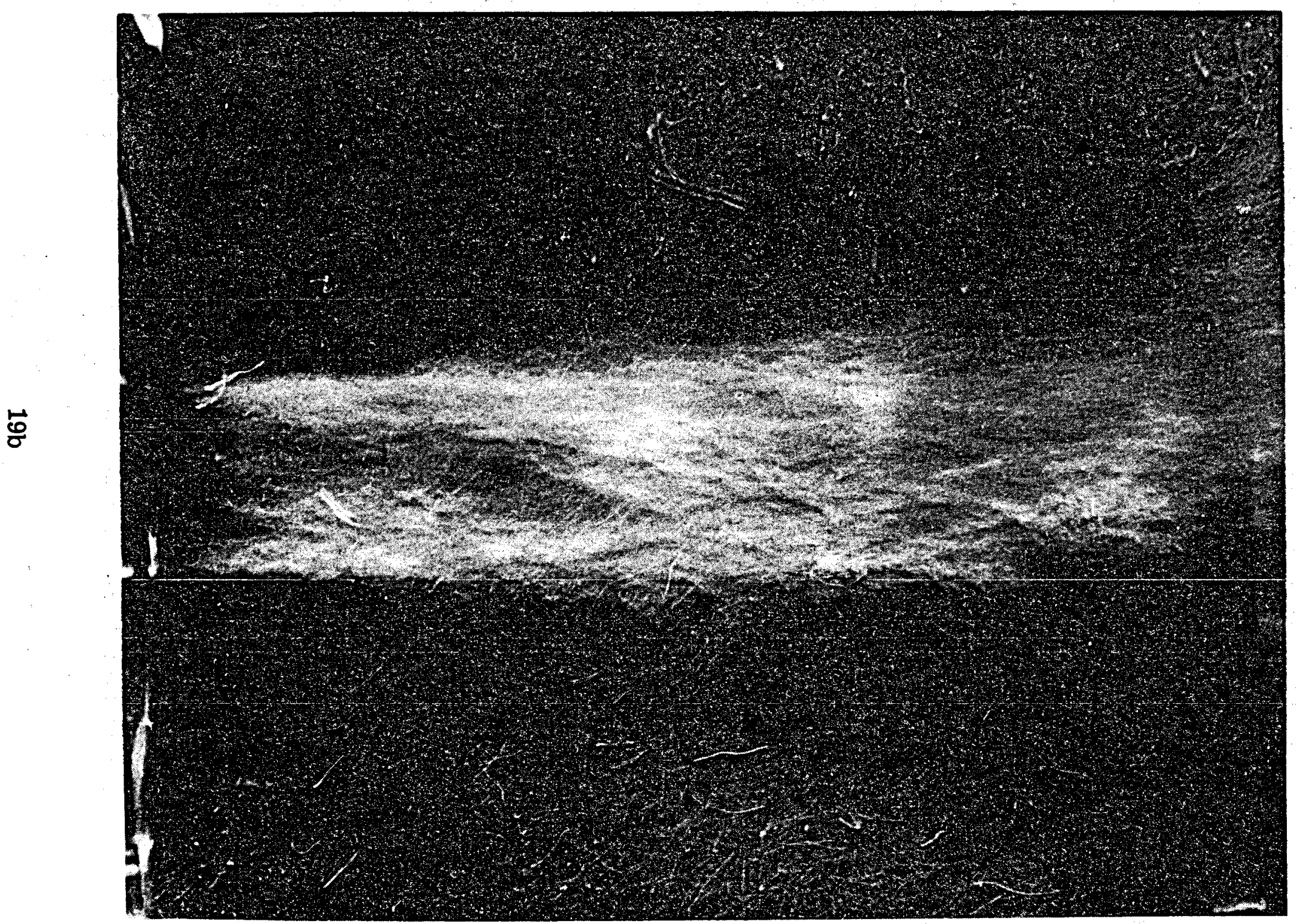

FIGURE 11 - SUBMERGED JET CAVITATION AS OBSERVED BY LONG DURATION EXPOSURE; photograph taken in High Speed Channel 
machined from large billets of clear acrylic. They had hemispherical contractions and exit diameters of 5.72 and $13.97 \mathrm{~cm}$ (2.25 and 5.5 in.), respectively. Means were provided for placing swirling vanes with adjustable angles of attack in the flow to the nozzle, and a vaned support for nozzle center bodies was furnished.

The flow exited from the nozzle in a free jet submerged to a depth of about $0.6 \mathrm{~m}$ ( $2 \mathrm{ft}$ ) from the jet centerline. The surrounding fluid was nominally still, but foam mats had to be placed on the free surface to minimize the entrainment of gas bubbles into eddies created by the action of the jet. Such bubbles interfered with observations of cavitation and could promote false gaseous cavitation inception, The cavitation index of the flow was controlled with the system pressure coupled with variations of the jet velocity.

The objectives of these experiments were to observe the cavitation patterns in the jet, to study the effect of the contraction ratio and of vortex generators on the inception of cavitation, and to examine the tendency of the turbulence in the jet's shear layer to structure with a preferred frequency. While the naked eye or a long exposure duration picture shows a quasiuniformly distributed cavitation "bubble" population on the periphery of the jet (Figure 11) a picture taken with a short duration exposure clearly showed a structuring; the cavitation vortices are concentrated in roughly equidistant regions (Figure 12). Clearly, cavitation had its inception in vortices located in the shear layer, the rotation axis of these vortices being apparently randomly distributed on the surface of contact between the jet and the ambient liquid (Figure 13). In a few instances where the velocity of the jet was slightly below cavitation inception conditions, a sudden "excitation" of the jet by an unknown 


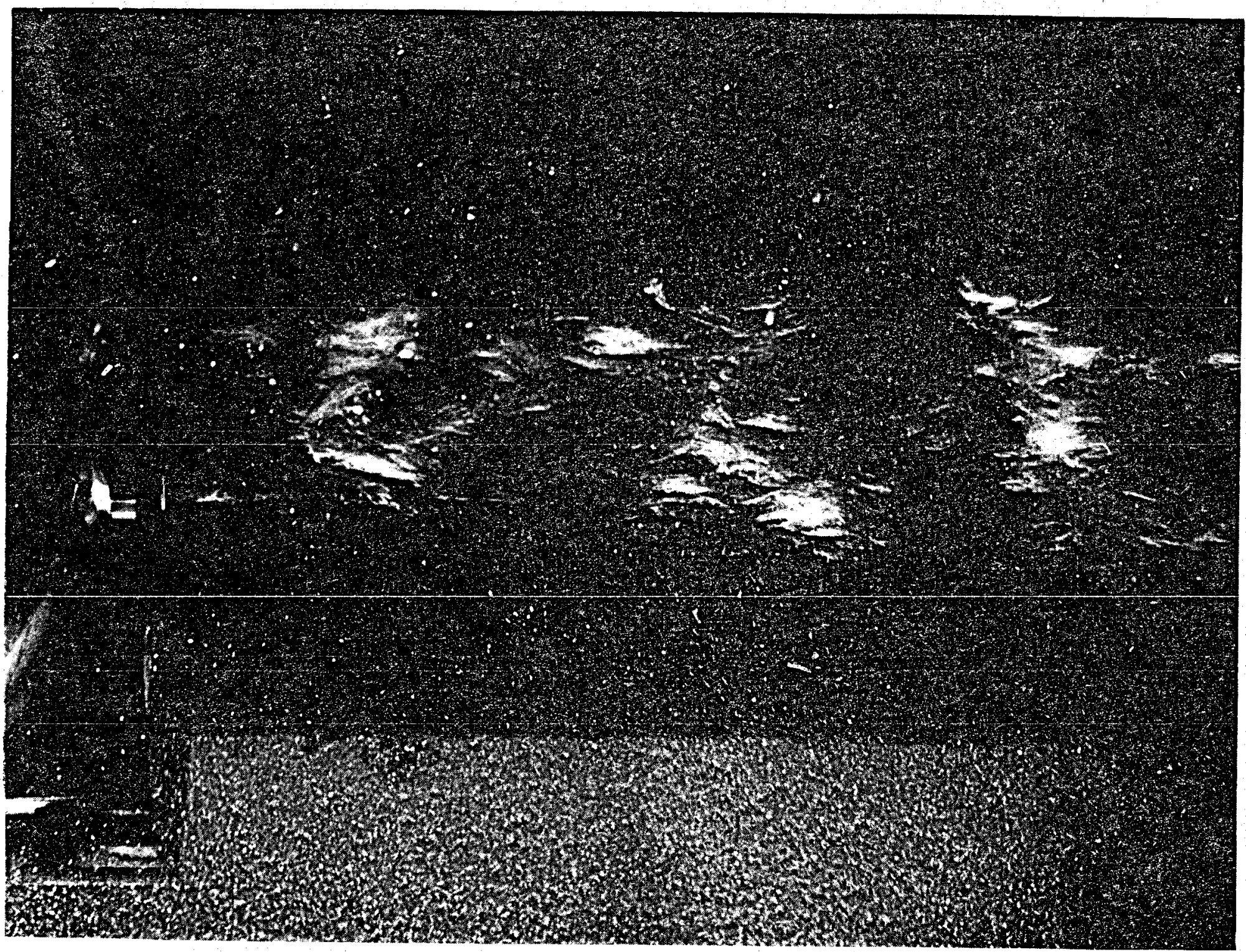

FÍGURE 12 - CAVITATION AS OBSERVED BY SHORT DURATION EXPOSURE, SHOWING JET STRUCTURING; photograph taken in High Speed Channel 


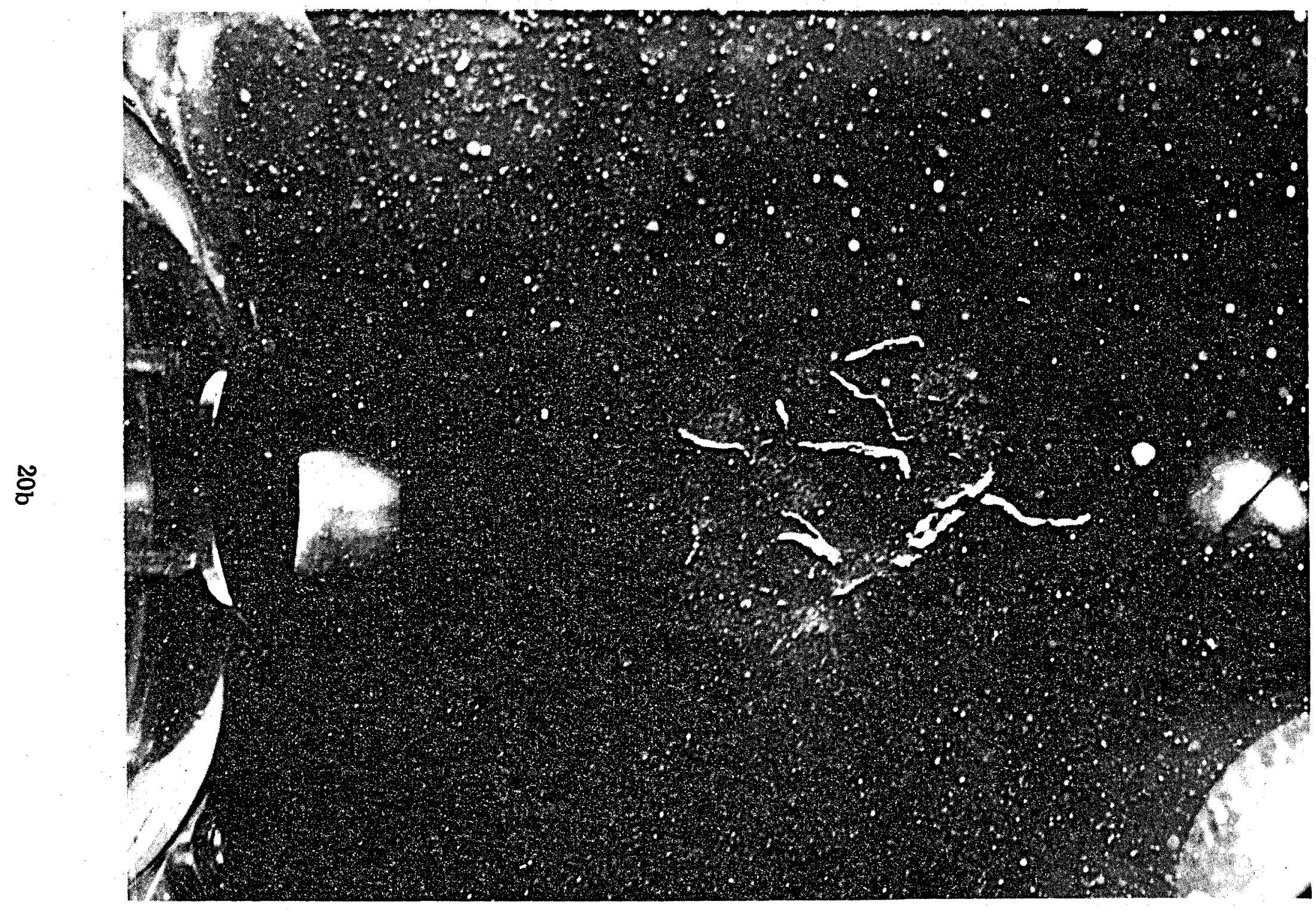

FIGURE 13 - SEGMENTS OF ROTATING CAVITATING VORTICES AT PERIPHERY OF JET; photograph taken in High Speed Channel 
means (probably related to vibrations of the entire channel) would generate a "puff" of a vortex ring which would come out loudly from the nozzle. We succeeded in capturing a photographic record of this event which clearly showed a ring vortex structure (Figure 14) similar to that observed later in our self-oscillating jets.

We designed and built two types of foils for the purpose of generating cavitating tip vortices. The first type had a constant span but was swept in order to obtain the desired load distribution (see Figure 15), The second had a flat shape (Figure 16) but had a variable span and was calculated using a special program developed for propeller design at HYDRONAUTICS. This second type of foil was satisfactory; however, for the first foil a flow detachment occurred well before cavitation inception. The pairs of foils were symmetrically located in the feed pipe as shown in Figure 10. They had, however, opposite angles of attack in order to generate tip vortices rotating in the same direction. A very nice visualization of these tip vortices rotating around each other was obtained, both under cavitating and noncavitating conditions (Figure 16). Injected dye was used to indicate the noncavitating vortices.

Cavitation inception was observed in the jet in the absence and in the presence of the swirling vanes, and when the contraction ratio was four (exit diameter $d=5.72 \mathrm{~cm}(2.25 \mathrm{in}$.); two $(d=13.97 \mathrm{~cm}(5.5 \mathrm{in.})$; and one $(d=27.9 \mathrm{~cm}$ (11 in.). Figure 17 summarizes all the results obtained. Two methods were used to determine inception. The acoustical detection, using a hydrophone, was more sensitive than the visual observation of "bubble" formation and gave higher values of the cavitation inception number. For $\mathrm{k}_{c}=1$, entrainment of air from the free surface, which was significantly reduced by the use of foam 


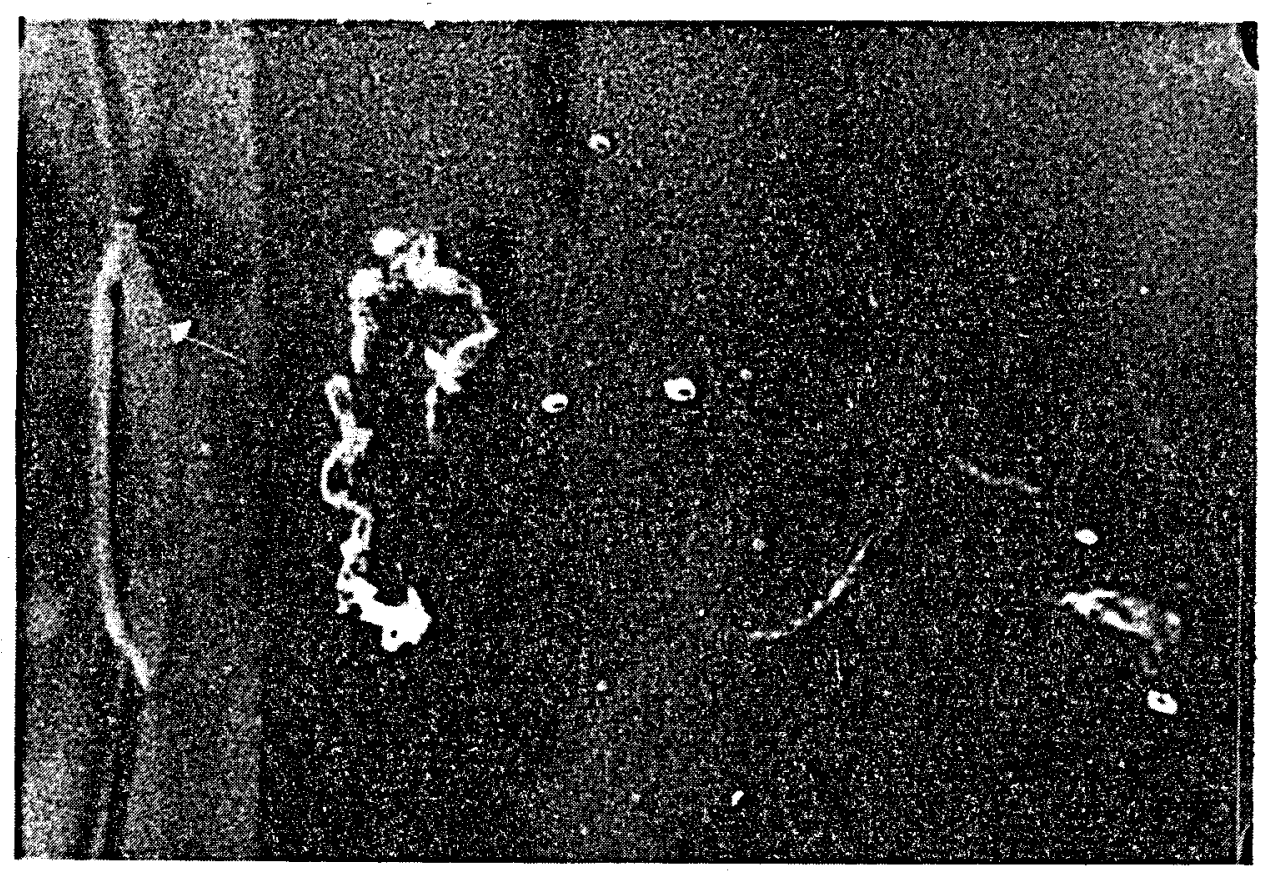

FIGURE 14 - RING VORTEX IN SUBMERGED JET FLOW; randomly generated during tests in High Speed Channel 


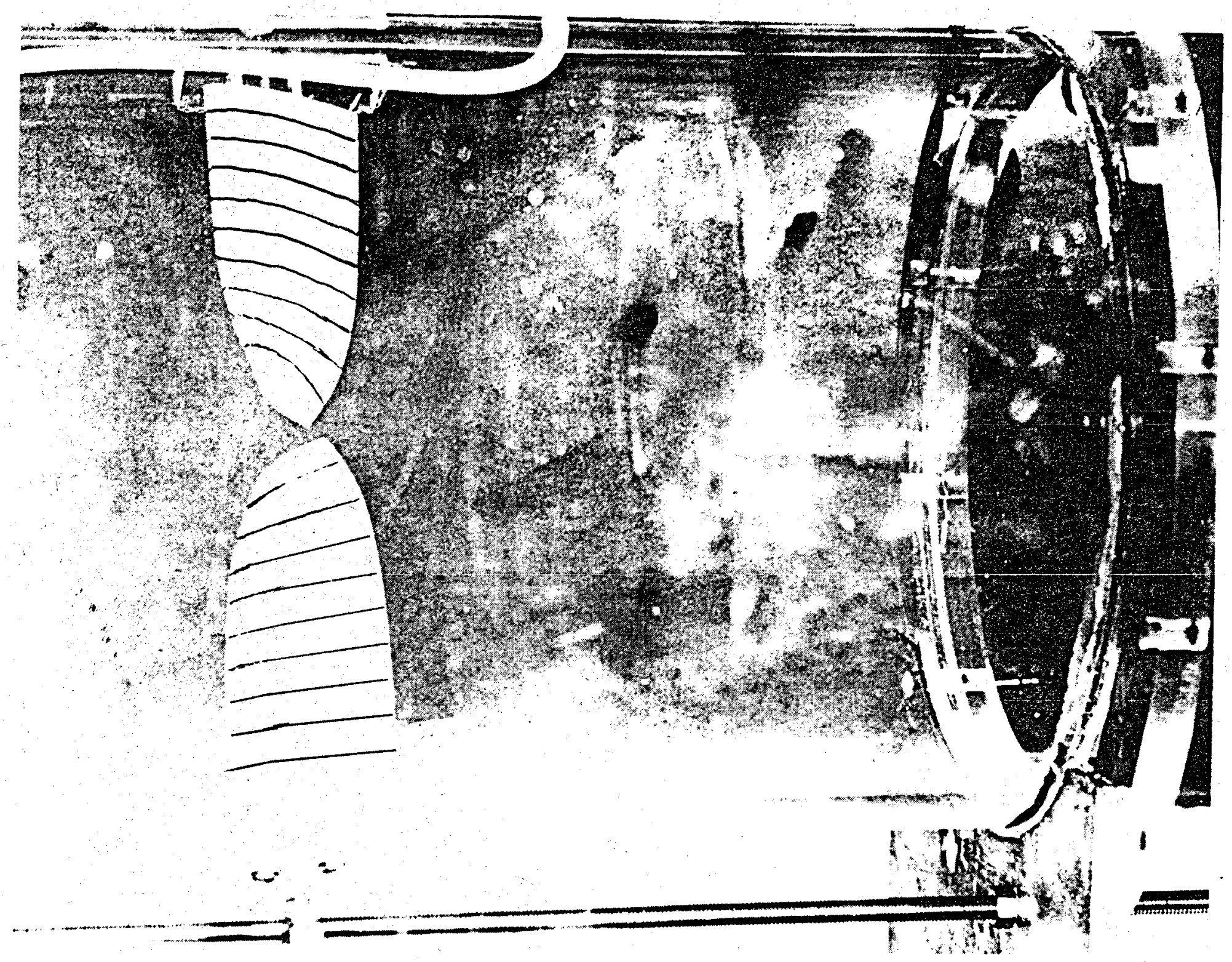

FIGURE 15 - TIP VORTEX GENERATING FOILS, with constant span and swept shape 


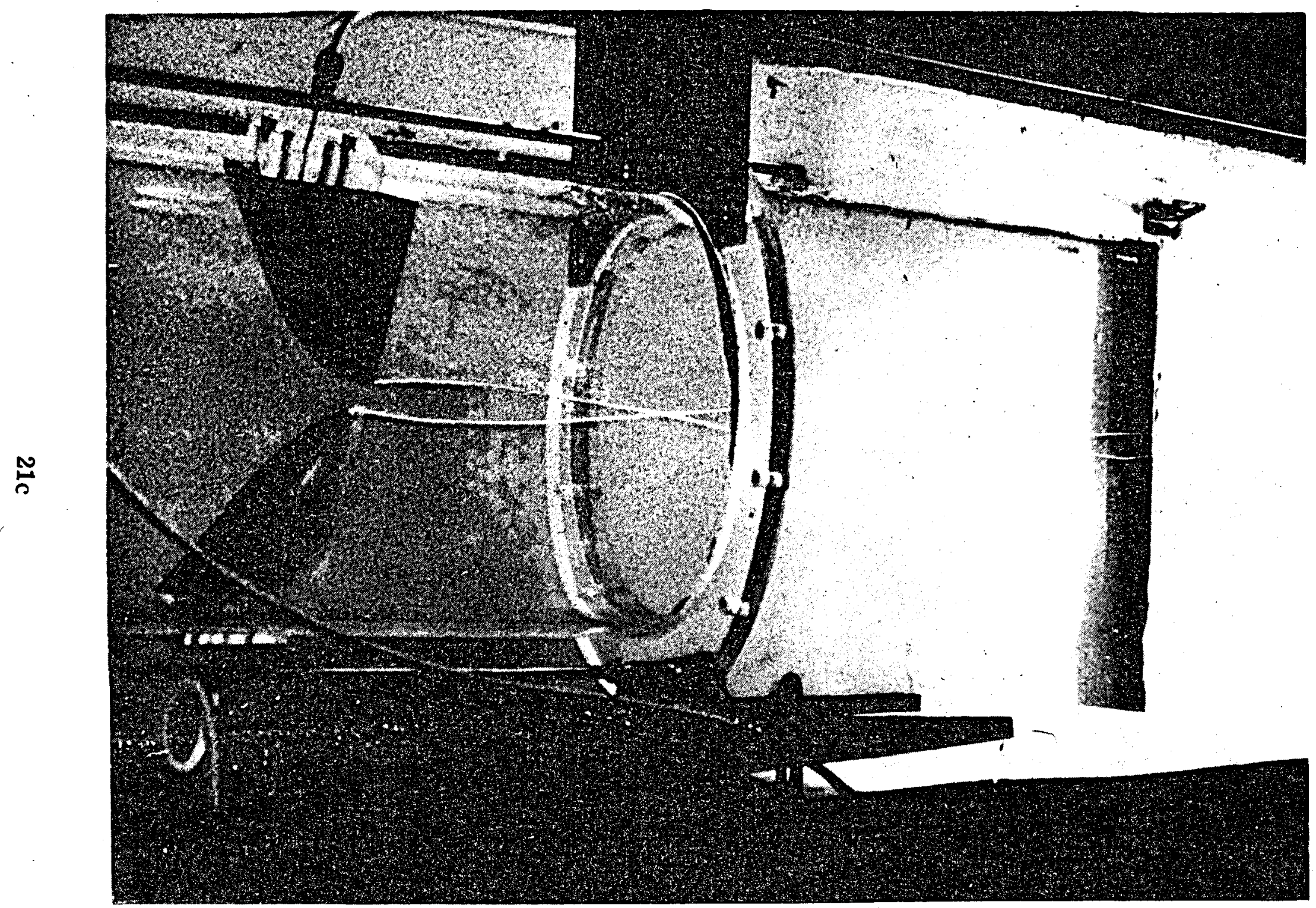

FIGURE 16 - TIP VORTEX GENERATING FOILS, with variable span and flat shape. Note tip vortices created by these foils. 


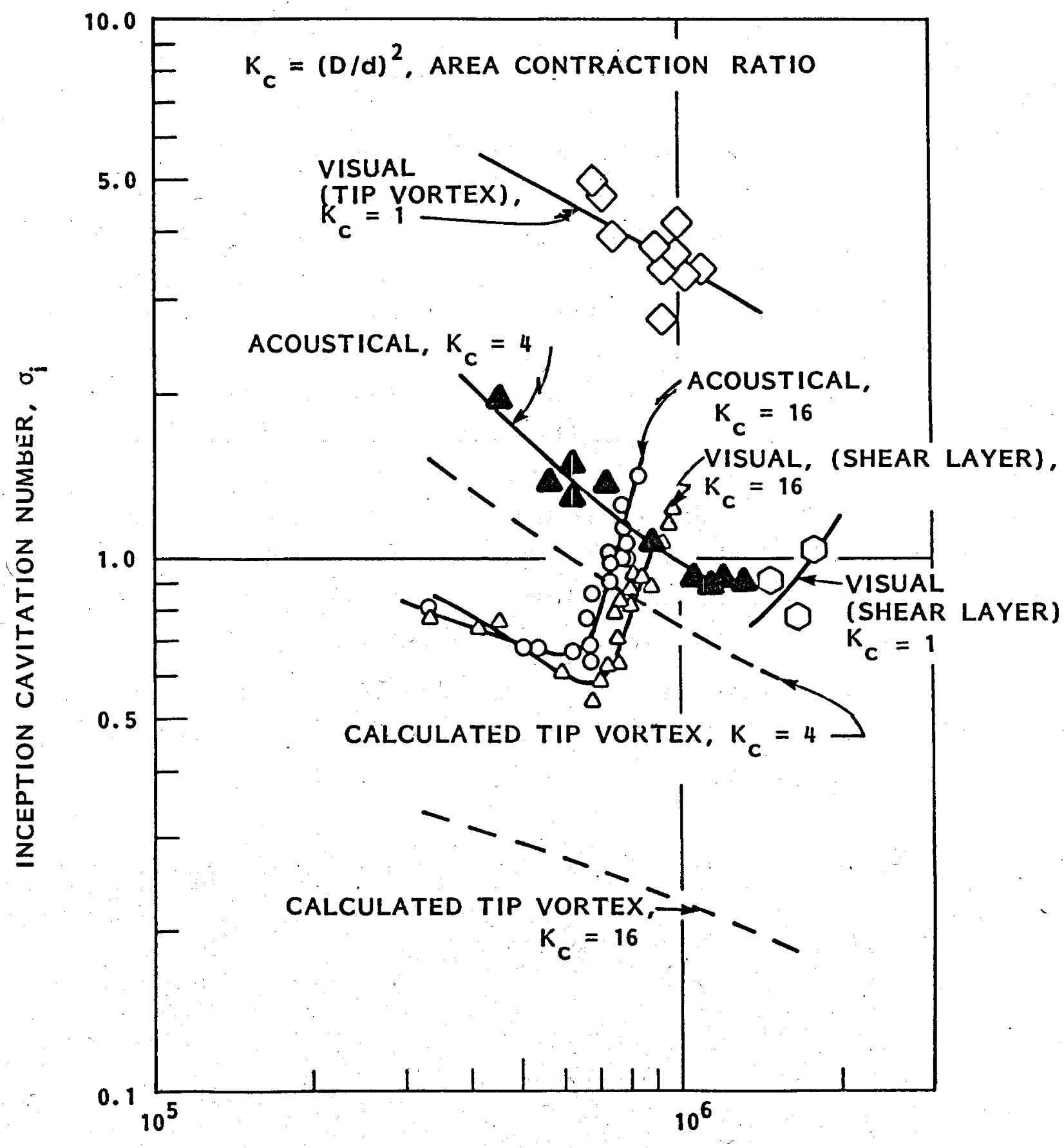

REYNOLDS NUMBER, $R e=V d / \nu$

FIGURE 17 - HIGH SPEED CHANNEL CAVITATION INCEPTION 
mats on the free surface, still prevented a good detection of cavitation inception.

The observation of tip vortex cavitation inception is only possible if this type of cavitation appears earlier than cavitation in the shear layer of the jet. This explains why the only visual tip vortex curve which appears in Figure 17 is for $\mathrm{k}_{c}=1$. In that case only cavitation inception in the tip vortex appeared very early $(3<\sigma<5)$ and could be distinguished. For the larger $k_{c}$ tests, the value of $\sigma$ at cavitation inception in the vortex center was smaller than the corresponding visual values of inception at the jet boundary represented in Figure 17 and thus the tip vortex inception could not be determined. These observations confirm the theoretical results presented in Section 2.2.1. The expected values of $\sigma$ at inception in the tip vortex for $k_{c}=1$ and 2 , are also shown in Figure 17 .

Tip vortex cavitation seems therefore to be a very efficient way of producing early cavitation inception only when the contraction ratio is small. 


\subsection{ORGAN PIPE ACOUSTICS THEORY}

\subsection{Single Pipe}

In order to deal with the propagation of acoustical waves in the somewhat complex geometry in the feed-tube for an extended nozzle (Figure 18) in a three-cone bit, let us consider first the behavior of a single pipe of constant area, A (see Figure 19). This pipe is such that the characteristic dimension of its cross-section is small compared to its length, $l$. We consider here the propagation in this pipe of an acoustic wave whose wave length is large compared to the characteristic size of the pipe section. The wave can be considered to be plane in the pipe, and in the absence of losses, the pressure and the velocity of the incident wave can be written in complex notations at the location, $x$, as follows:

$$
\begin{gathered}
p_{i}=B e^{j(\omega t-k x)} \\
u_{i}=\frac{p_{i}}{\rho c}=\frac{B}{\rho c} e^{j(\omega t-k x)}
\end{gathered}
$$

where: $\rho \partial u_{i} / \partial t=-\partial p_{i} / \partial x ; \omega$ is the angular frequency $(\omega=$ $2 \pi f ; f$, frequency); $k$ is the wave number $(k=2 \pi / \lambda ; \lambda$, wave length); $\rho$ is the liquid density; and $c$ is the speed of sound in the liquid.

In reality, the acoustic pressure and the perturbation velocity at $x$ are not $p_{i}$ and $u_{i}$, since due to changes in the acoustic characteristics at the pipe exit (more precisely defined below) an acoustic wave reflected at the pipe exit adds its contribution, $p_{r}$ and $u_{r}$, to $p_{i}$ and $u_{i}$ : 


$$
\left.\begin{array}{c}
P_{I}=D e^{j(\omega t+k x)} \\
u_{I}=-\frac{P_{I}}{\rho C}=-\frac{D}{\rho C} e^{j(\omega t+k x)}
\end{array}\right\}
$$

The acoustical pressure and velocity at $\mathrm{x}$ are therefore:

$$
\left.\begin{array}{l}
\dot{p}(x)=p_{r}+p_{i}, \\
u(x)=u_{r}+u_{i} .
\end{array}\right\}
$$

The end conditions at the location $\mathrm{x}=0$ and $\mathrm{x}=\ell$ are very important in determining the acoustical behavior of the pipe. Namely, one must verify that the pressure and the flow rate at $x=0$ are equal to those existing in the upstream system at the pipe inlet. Similarily, at $x=\ell$ the pressure and flow rate are equal to those at the same location in the system following the pipe.

In order to simplify the analysis it is very convenient to introduce the notion of acoustical impedance (see, for instance References 14, 15). This is similar to the electrical approach; the electrical analogy being between acoustic pressure and electrical potential, velocity and current intensity. The complex acoustical impedance, $Z$, is defined as:

$$
Z(x)=p(x) / A u(x)
$$

The simplification seems clear when we notice that the two conditions at each end of the tube can be replaced by a single one. The acoustical impedance at $\mathrm{x}=0$ must match the imposed upstream . 


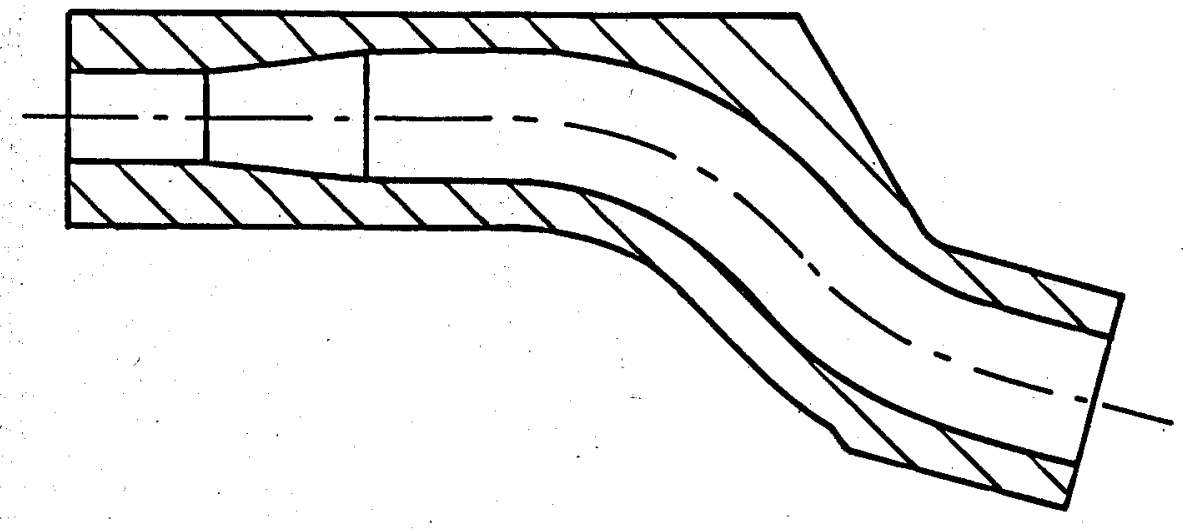

FIGURE 18 - TYPICAL EXTENDED NOZZLE FEED-TUBE FOR THREE-CONE ROLLER BIT 

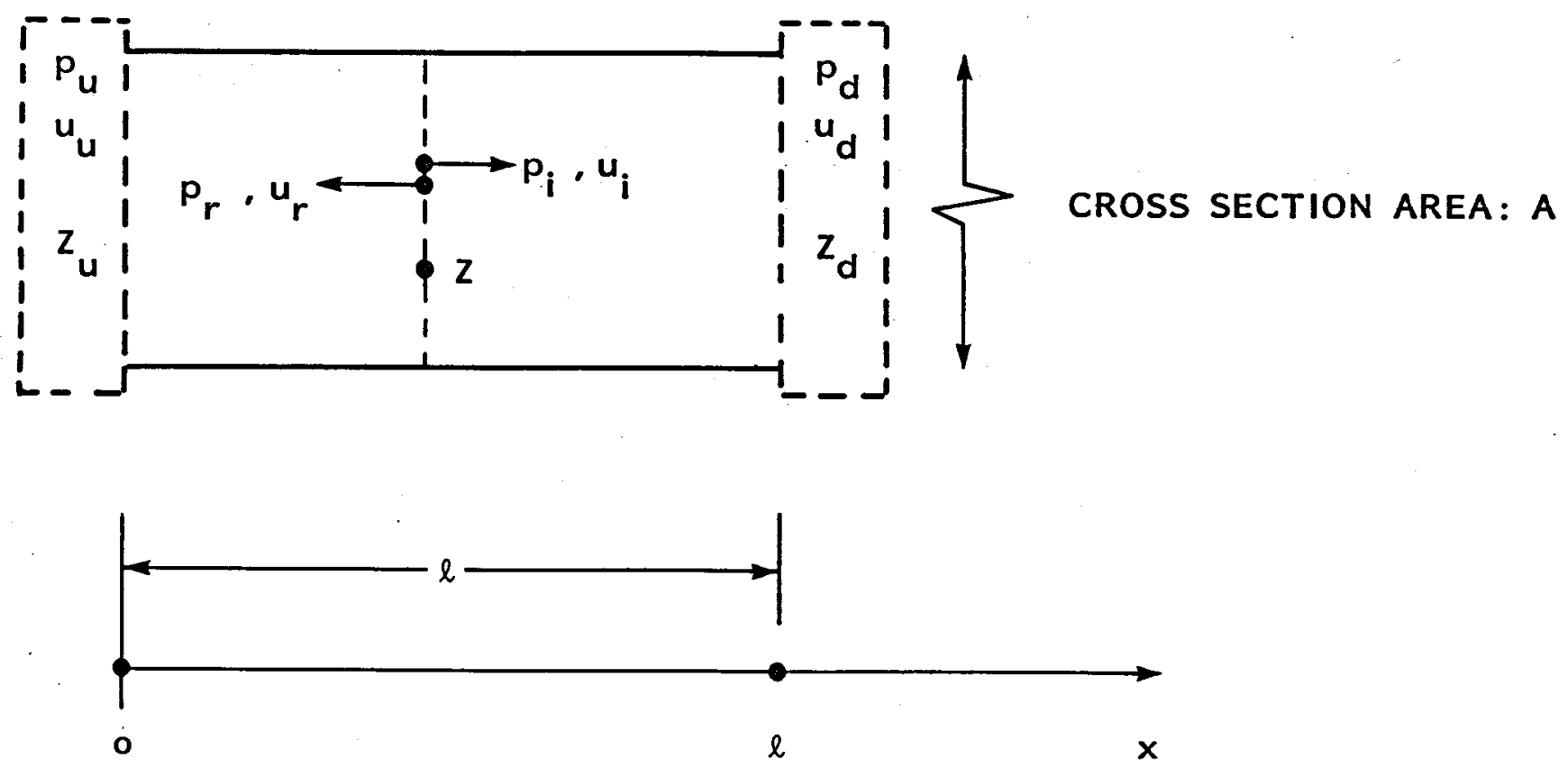

FIGURE 19 - PARAMETERS FOR ANALYSIS OF ORGAN-PIPE ACOUSTICS 
impedance, $Z_{0}$, and the impedance at $x=l$ must match the imposed exit impedance, $z_{e}$.

$$
\left.\begin{array}{l}
z(0)=z_{0}, \\
z(l)=z_{e^{\prime}}
\end{array}\right\}
$$

Replacing Equations [42] in the general expression of $z(x)$ :

$$
Z(x)=\frac{\rho c}{A} \frac{B e^{-j k x}+D e^{j k x}}{B e^{-j k x}-D e^{j k x}}
$$

one obtains the following relationship betwen $k, z_{0}, z_{e}$, and the pipe and the liquid characteristics:

$$
z_{0}=z_{c} \frac{z_{e}+j z_{c} \tan (k l)}{z_{c}+j z_{e} \tan (k l)}
$$

where $z_{c}$ is the "characteristic impedance" of the pipe:

$$
Z_{c}=\rho c / A
$$

Knowing the pipe characteristic, $z_{c}$, and the end conditions, $z_{0}$ and $Z_{e}$, one can solve for $k$. Table 1 summarizes the values of particular impedances for the most common end conditions.

The preceding equations can be written in a pore general and precise way by accounting for an attentuation or amplification of the wave amplitude with time. This can be expressed in terms of a complex wave number, $\bar{k}$, in which case Equation [44] becomes: 
TABLE 1

SOME TYPICAL END IMPEDANCES

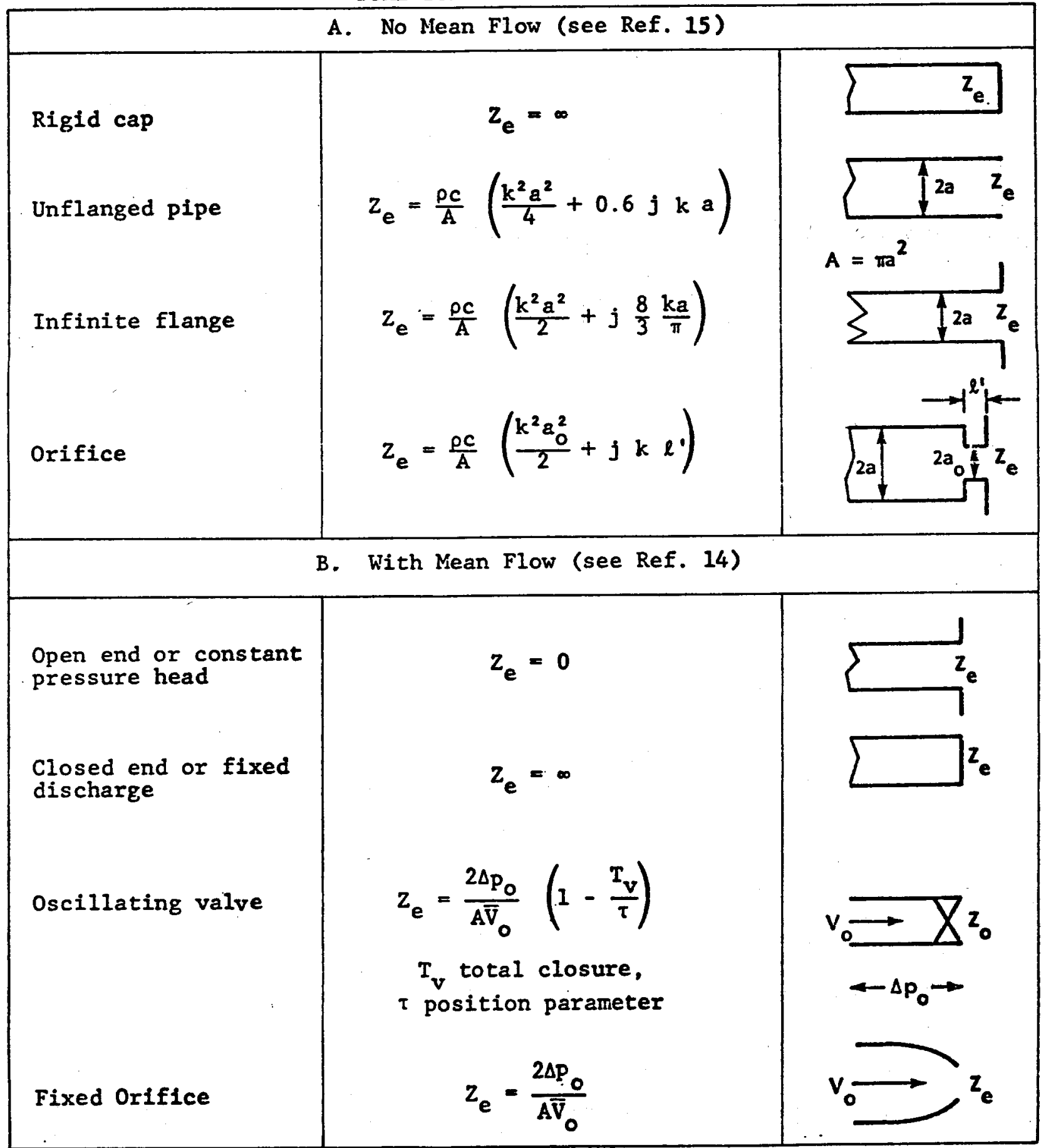




$$
z_{0}=z_{c} \frac{z_{e}+z_{c} \tanh (\hat{k} \ell)}{z_{c}+z_{e} \tanh (\hat{k} \ell)}
$$

Let us now consider, as an illustration of the problem, the case where the upstream end of the pipe is a constant head plenum. Then, $z_{0}=0$, and we can write in the general case:

$$
\tanh (\overline{\mathrm{k}} l)=-\mathrm{Z}_{\mathrm{e}} / \mathrm{Z}_{\mathrm{c}}
$$

One immediately finds the classical results for an organ-pipe when $z_{e}$ is either zero or infinite (closed or open end pipe)

$$
\begin{aligned}
& \dot{k l}=(2 n+1) \frac{\pi}{2}, \text { if } z_{e}=\infty, \\
& k l=2 n \frac{\pi}{2}, \text { if } z_{e}=0 .
\end{aligned}
$$

or expressed in terms of wave lengths:

$$
\left.\begin{array}{l}
\ell=\frac{2 n+1}{4} \lambda, \text { if } z_{e}=\infty, \\
\ell=\frac{n}{2} \lambda, \text { if } z_{e}=0
\end{array}\right\}
$$

For a pipe with no flow and an infinite flange (or unflanged) (see Table 1) expressions [49] are corrected by terms accounting for the end orifice condition:

$$
\begin{aligned}
& \ell+0.6 a=\frac{n}{2} \lambda, \text { unflanged pipe, } \\
& \ell+\frac{8}{3 \pi} a=\frac{n}{2} \lambda, \text { infinite flange. }
\end{aligned}
$$




\subsection{Single-Pipe Modes and Optimum Mach Number}

The most interesting case for us is that for which, besides the upstream condition $\mathrm{z}_{0}=0$, a pressure drop, $\Delta \mathrm{p}_{0}$ exists across an orifice located at the downstream end of the pipe, which produces in the pipe a flow of mean velocity, $V_{0}$. The exit impedance is then:

$$
z_{e}=2 \Delta p_{o} / V_{o} A
$$

This exit impedance determines whether the pipe behaves as a closed end or an open end pipe [48]. This can be expressed in terms of the geometry of the pipe and of flow characteristics. Note that:

$$
\left.\begin{array}{c}
\Delta \mathrm{p}_{0}=\frac{1}{2} \rho\left(\mathrm{v}_{j}{ }^{2}-\mathrm{v}_{0}{ }^{2}\right) \\
\Delta \mathrm{p}_{0}=\frac{1}{2} \dot{\rho} \mathrm{v}_{0}^{2}\left[\left(\frac{A}{A_{j}}\right)^{2}-1\right]
\end{array}\right\} \text {, }
$$

where $V_{j}$ and $A_{j}$ are, respectively, the jet velocity and section area. We can write:

$$
\frac{Z_{e}}{Z_{c}}=\frac{2 \Delta p_{o}}{P C V_{o}}=\frac{V_{0}}{c}\left[\left(\frac{A}{A_{j}}\right)^{2}-1\right] .
$$

and, in terms of the jet Mach number, $M=V_{j} / c$, this becomes:

$$
-\tanh (\bar{k} l)=\frac{Z_{e}}{Z_{c}}=M\left[\frac{A}{A_{j}}-\frac{A_{j}}{A}\right] .
$$


Separating the imaginary from the real part one finds for the imaginary part:

$$
\left.\begin{array}{l}
k \ell=n \pi / 2, \\
\ell / \lambda=n / 4,
\end{array}\right\}
$$

which shows that the pipe can act either as an open duct ( $n$ : even) or a closed end ( $n$ : odd). The determination of $n$ is obtained with the real part which depends on the value of $\mathrm{z}_{\mathrm{e}} / \mathrm{Z}_{\mathrm{c}}$ in Equation [55]. One obtains for the imaginary part of $\vec{k}(\hat{k}=$ $k+i h)$ :

$$
h=\frac{1}{2 l} \ln \left[(-1)^{n} \frac{1-Z_{e} / Z_{c}}{1+Z_{e} / Z_{c}}\right]
$$

which shows that $n$ is even when $Z_{e}$ is smaller than $Z_{c}$, and $n$ is odd when $z_{e}$ is greater than $z_{c}$. Therefore:

$$
\left.\begin{array}{l}
l=n \lambda / 2 ; \text { if } M\left[\frac{A}{A_{j}}-\frac{A_{j}}{A}\right] \leq 1, \\
l=(2 n+1) \lambda / 4, \text { if } M\left[\frac{A}{A_{j}}-\frac{A_{j}}{A}\right] \geq 1 .
\end{array}\right\}
$$

This condition can be simplified to the following, when $\left(A_{j} / A\right)^{2}$ is negligible in comparison to unity:

$$
\left.\begin{array}{l}
\ell=n \lambda / 2 ; \text { if } \frac{A}{A_{j}}<M^{-1} \\
\ell=(2 n+1) \lambda / 4 ; \text { if } \frac{A}{A_{j}}>M^{-1}
\end{array}\right\}
$$


For a Mach number of 0.1 , a diameter contraction ratio of 3.2 is the separation line between the two cases in [59].

Now, as often emphasized elsewhere in this report, optimum performances of an organ-pipe self-excited jet were obtained when the natural frequencies of the pipe and of the free jet (or their harmonics) were matched together. Since the natural frequency of the jet is known and corresponds to a Strouhal number of about 0.3, the optimum performances for a given pipe are directly related to the jet Mach number. Indeed we can write:

$$
s_{d}=\frac{f d_{j}}{V_{j}}=\frac{f d_{j}}{c} \cdot \frac{c}{v_{j}}
$$

and since $\lambda=c / f$ :

$$
S_{d}=\frac{d}{d} \cdot M^{m-1}
$$

Accounting for [59], Equation [61] can be written

$$
M=s_{d}^{-1} \cdot \frac{d_{j}}{\ell} \cdot K_{n}
$$

with $k_{n}$, a "mode parameter", given for each "mode number", $n$, as :

$$
\begin{gathered}
K_{n}=\frac{n}{2} \text {; if } \frac{A}{A_{j}}<M^{-1}, \\
K_{n}=\frac{2 n-1}{4} \text {; if } \frac{A}{A_{j}}>M^{-1} \text {. }
\end{gathered}
$$

Expression [62] is written for the case where no end correction on the pipe length is applied. In such a case $k_{n}$ is to be replaced by a corrected value: 


$$
\mathrm{K}_{\mathrm{n}}=\mathrm{K}_{\mathrm{n}} /(1+\varepsilon)
$$

For an example, in the case of an unflanged pipe, $\varepsilon$ takes the value $0.6 \mathrm{~d} / \mathrm{l}$.

Equation [62] is represented in Figure 20 for two values of $\mathrm{s}_{\mathrm{d}}$ and for various modes, $\mathrm{n}$. Comparisons with experimental measurements plotted on the same figure show a very good agreement.

\subsection{Case of a Series Pipe System}

In the case of a series of pipes of different cross sections (Figure 21) each of the components of the system can be treated acoustically as a single pipe with given end impedances (see Section 3.1). The relation between these end impedances is the same as Equation [44], and can be written for the $i-t h$ pipe in a series system as:

$$
z_{o}^{i}=z_{c}^{i} \frac{z_{e}^{i}+j z_{c}^{i} \tan k l_{i}}{z_{c}^{i}+j z_{e}^{i} \tan k l_{i}} .
$$

In addition we have the relations:

$$
z_{0}^{i}=z_{e}^{i-1}
$$

Let us consider as an illustration the case of two pipes in series with $z_{0}^{i}=0$ (Figure 22). If we write:

$$
\frac{Z_{e}^{1}}{Z_{c}^{1}}=\frac{Z_{e}^{1}}{\rho c / A_{1}}=\alpha_{1}+j B_{1} \text {. }
$$

and substitute [67] into [65], we obtain 


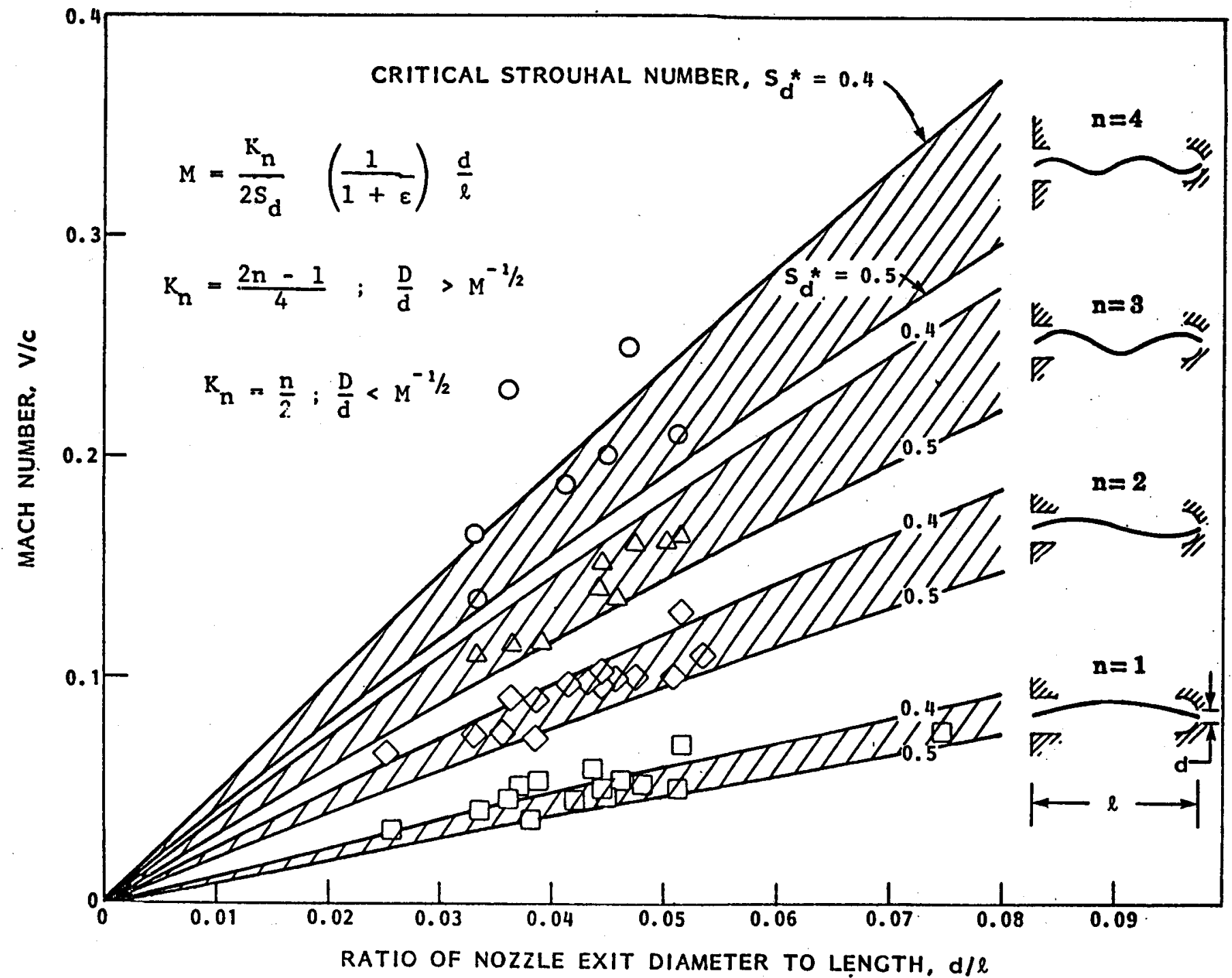

FIGURE 20 - CORRELATION OF OPTIMUM MACH NUMBERS AND GEOMETRY OF ORGAN-PIPE CAVIJET $\odot$ NOZZLES 


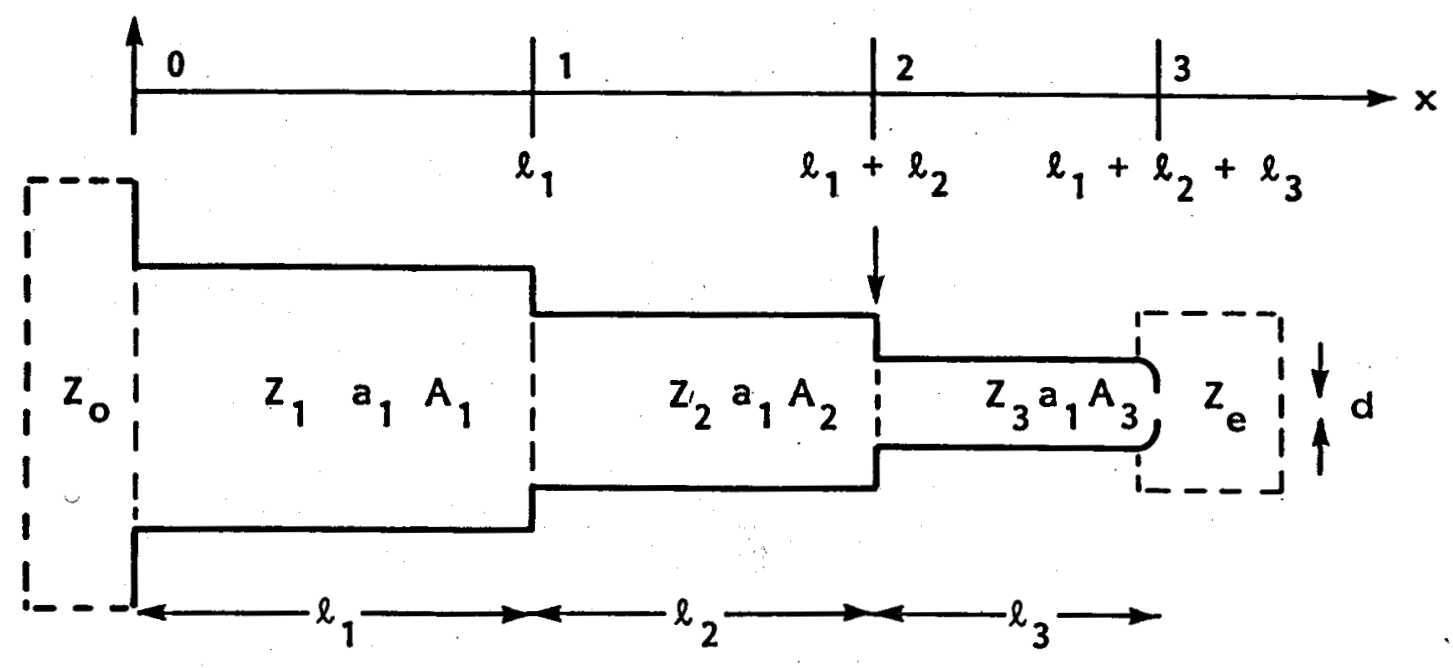

FIGURE 21 - ORGAN PIPE WITH SERIES OF DIFFERENT CROSS SECTIONS (pipe area: $A_{i}$; pipe radius: $a_{i}$ )

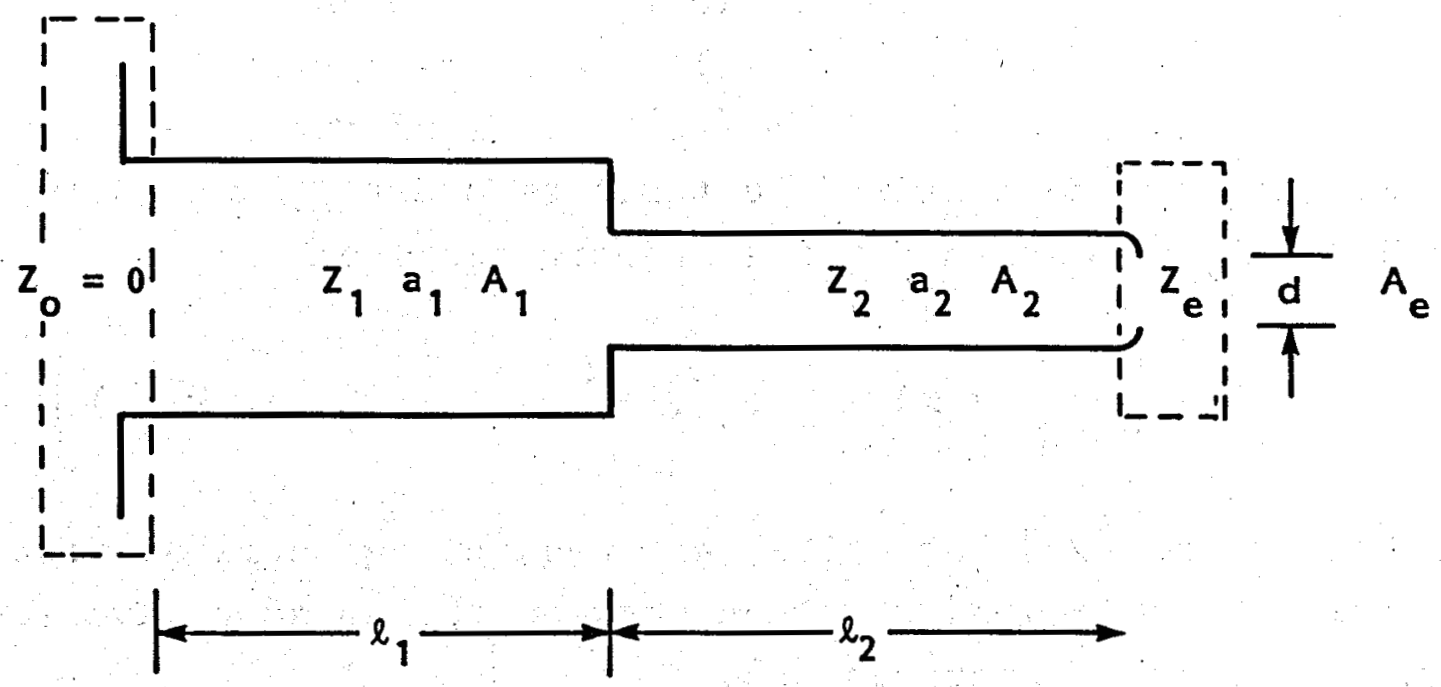

FIGURE 22 - ORGAN PIPE WITH TWO CROSS SECTION AREAS 


$$
\left(1-\beta_{1}^{2}-\alpha_{1}^{2}\right) \operatorname{tank} l_{1}+\beta_{1}\left(1-\tan ^{2} k l_{1}\right)=0 \text { [68] }
$$

The terms $\alpha_{1}$ and $\beta_{1}$ can be obtained by applying again [65] for the second pipe of length $\ell_{2}$.

$$
\begin{aligned}
& \beta_{1}=\frac{A_{1}}{A_{2}} \frac{\left(1-\beta_{2}^{2}-\alpha_{2}^{2}\right) \operatorname{tank} l_{2}+\beta_{2}\left(1-\tan ^{2} k l_{2}\right)}{\left(1-\beta_{2} \operatorname{tank} l_{2}\right)^{2}+\alpha_{2}^{2} \tan ^{2} k l_{2}} .
\end{aligned}
$$

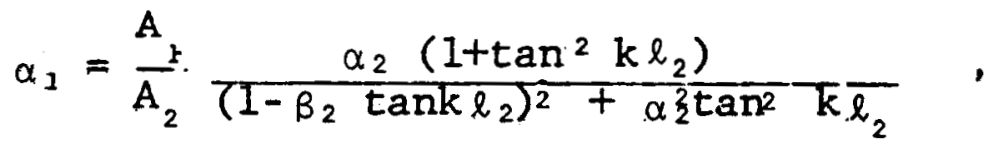

where $Z_{e}^{2}=\frac{\rho c}{A_{2}}\left(\alpha_{2}+j \beta_{2}\right)$

In all cases which are of interest for us $\alpha_{2}$ is either zero or negligible. Indeed as seen in Table $1, \alpha_{2}$ (when not zero) is proportional to $\mathrm{k}^{2} \mathrm{~d}_{j}{ }^{2}$ or $\left(\mathrm{d}_{j} / l_{2}\right)^{2}$. As a result $\alpha_{1}$ is also negligible, since as seen from [70], $\alpha_{1}$ is of the same order as $\alpha_{2}$. Similarily, neglecting the terms of order $\beta_{2}^{2}$ and $\alpha_{2}^{2}$ in Equation [69] we obtain for the leading terms of $\beta_{1}$ :

$$
B_{1} \simeq \frac{A_{1}}{A_{2}}\left[\tan k_{2}+B_{2}\left(1-\tan ^{2} k l_{2}+2 \tan k_{2}\right)\right]
$$

By substituting [72] into [68], and neglecting terms as discussed, we obtain the following relation between the unknown wave length, $\lambda$, and the pipe characteristics:

$$
\frac{2 \pi}{\lambda} \ell_{1}=n \pi-\frac{A}{A_{2}}\left[\tan \frac{2 \pi}{\lambda} \ell_{2}+B_{2}\left(1-\tan ^{2} k_{2}+2 \tan k_{2}\right)\right]
$$

This equation can be solved graphically by looking, for example, 
for the intersection between the curve:

$$
f\left(\lambda^{*}\right)=\frac{A}{A_{2}}\left[\frac{\lambda^{*}}{\pi} \tan \frac{2 \pi}{\lambda^{*}}+\beta_{2}\left(1-\tan ^{2} \frac{2 \pi}{\lambda^{*}}+2 \tan \frac{2 \pi}{\lambda^{*}}\right)\right] .
$$

and the line:

$$
g\left(\lambda^{*}\right)=\mathrm{n} \cdot \lambda^{*}-2 l_{1}^{*}
$$

where:

$$
\ell_{1}^{*} \equiv \ell_{1} / l_{2}, \lambda^{*} \equiv \lambda / l_{2}
$$

Figure 23 shows the intersection between these two curves for an unflanged pipe $\left(B_{2}=0.6 \mathrm{ka}\right)$. Several values of the ratio $l_{1} *$ and of the mode nimber, $n$, are considered. The value of the abscissas is then the ratio between the wave length and $l_{2}$.

If in addition we assume that tan $\mathrm{kl}_{2}$ is small, an approximation of Equation [73] is :

$$
\text { n } \frac{\lambda}{2}=\ell_{1}+\frac{A_{1}}{A_{2}}\left(\ell_{2}+\frac{B_{2}}{k}\right) \text {. }
$$

With these assumptions we can solve the case of $n$ pipes; for example for three pipes with unflanged ends:

$$
\text { in } \frac{\lambda}{2}=l_{2}+\frac{A}{A_{2}} l_{2}+\frac{A}{A_{2}}\left(l_{3}+0.6 a_{3}\right) \text {, }
$$

and for an infinite flange:

$$
n \frac{\lambda}{2}=l_{1}+\frac{A_{1}}{A_{2}} l_{2}+\frac{A}{A_{2}}\left(l_{3}+\frac{8}{3 \pi} a_{3}\right) \text {. }
$$




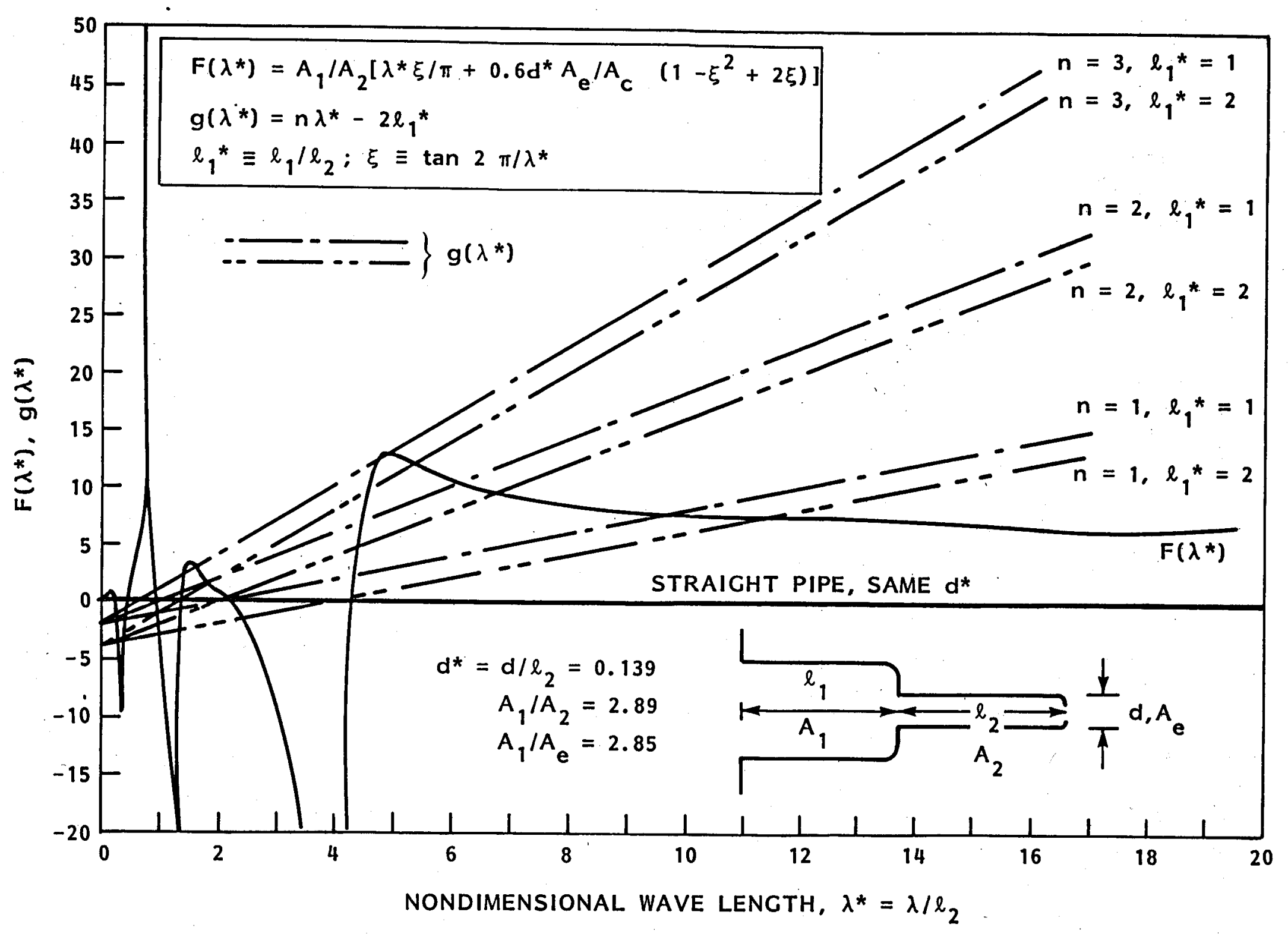

FIGURE 23 - GRAPHICAL SOLUTION FOR THE RESONANCE OF A TWO-STEP ORGAN PIPE

\section{r}




\subsection{ROCK EROSION TESTS}

The feasibility of jet-cutting rock under simulated downhole conditions with CAVIJET nozzles had been demonstrated earlier in tests on Indiana limestone (Reference 1). We pursued rock cutting experiments in the present program in order to learn more about the principles of cavitating jet erosion, particularly with regard to enhanced self-resonating nozzle designs that we had begun to develop (Reference 2).

We found that it was necessary to employ rock specimen calibration procedures, in many cases, to help reduce the data scatter so that meaningful comparisons between nozzle types could be made. Otherwise, comparisons had to be limited to runs made on the same specimen surface. Both stationary (hole drilling) and translating (slot cutting) erosion modes were used.

Several test sequences were run on specimens of Sierra white granite to examine the effectiveness of cavitating jets for eroding the hard impermeable rock found in geothermal environments and to serve as a guide in setting quantitative goals for follow-on work. Berea sandstone and Indiana limestone specimens were also used to quantify cutting performance changes attributed to nozzle size effects and other operating parameters.

\subsection{Sierra White Granite Tests at DRL}

The first rock erosion tests in the present program were successfully conducted within the Wellbore simulator at the Drilling Research Laboratory (DRI) of Terra Tek, Incorporated, on April 22-24, 1980. A total of 61 runs were made on two specimens of Sierra white granite; 27 runs on the first and 34 runs on the second specimen. Test variables for these stationary runs included nozzle pressure drop, cavitation 
number and nozzle type and size. Cross sections of the four test nozzles are depicted in Figure 24.

Considerations of available flow rate $(10.1 \mathrm{l} / \mathrm{s}$ (160 gpm)) and pressures (103.4 $\mathrm{MPa}$ (15,000 psi) pump and $51.7 \mathrm{MPa}(7,500$ psi) ambient) led NL/HYCALOG to design and furnish a new twonozzle tool for these tests as shown schematically in Figure 25. Another change was a vastly improved ratcheting mechanism which was created by DRL specially for these tests to ameliorate difficulties encountered during earlier testing ( 1 ). This new ratcheting device worked perfectly, completely eliminating the frequent malfunctions in indexing the tool which plagued us throughout those earlier efforts in the Wellbore Simulator. Otherwise the test configuration was essentially the same as that used during the first phase of this program (SANDIA Contract No. 07-7067); for details of the test configuration and procedures, see Reference 1.

On the first specimen, both CAVIJET cavitating jet nozzles had an orifice diameter of $5.2 \mathrm{~mm}(0.204 \mathrm{in.})$. One of these had the "plain" CAVIJET nozzle configuration; the other was the new PULSER type of CAVIJET cavitating jet nozzle which consisted of tandem orifices with an intervening, resonant chamber (Figure 24b).

To further examine size-scaling effects, the second rock was exposed to hole cutting tests by two CAVIJET nozzles, one having an orifice diameter of $2.6 \mathrm{~mm}(0.101 \mathrm{in.})$ and the second $7.1 \mathrm{~mm}(0.281 \mathrm{in.})$. Unfortunately, due to erosion damage on these nozzles and their subsequent repair, as discussed below, the configuration of their orifices was only approximately that of the original design. 


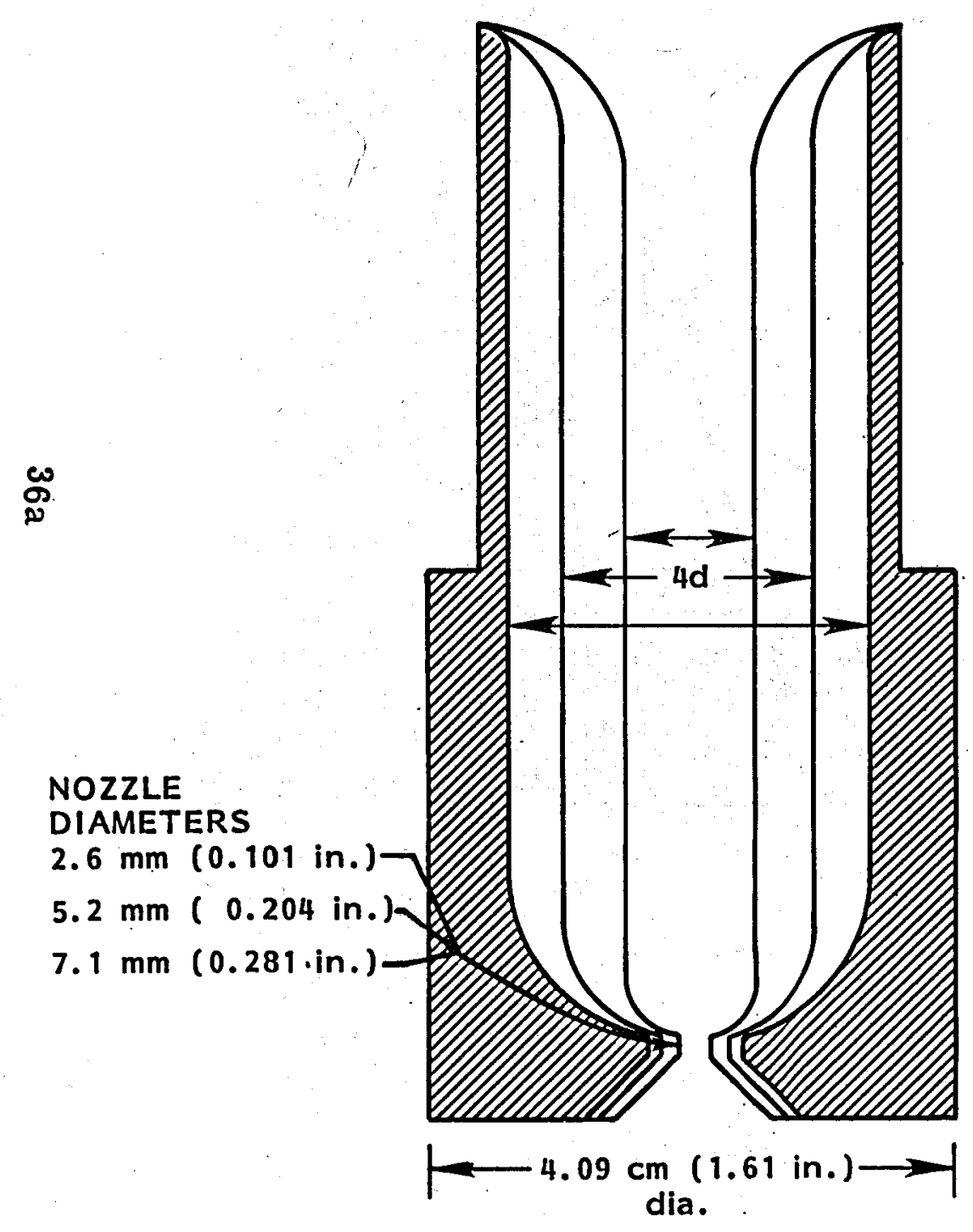

a. PLAIN CAVIJETS

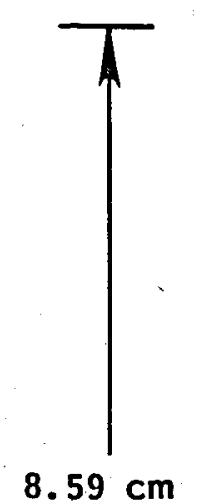

(3. 38 in.)
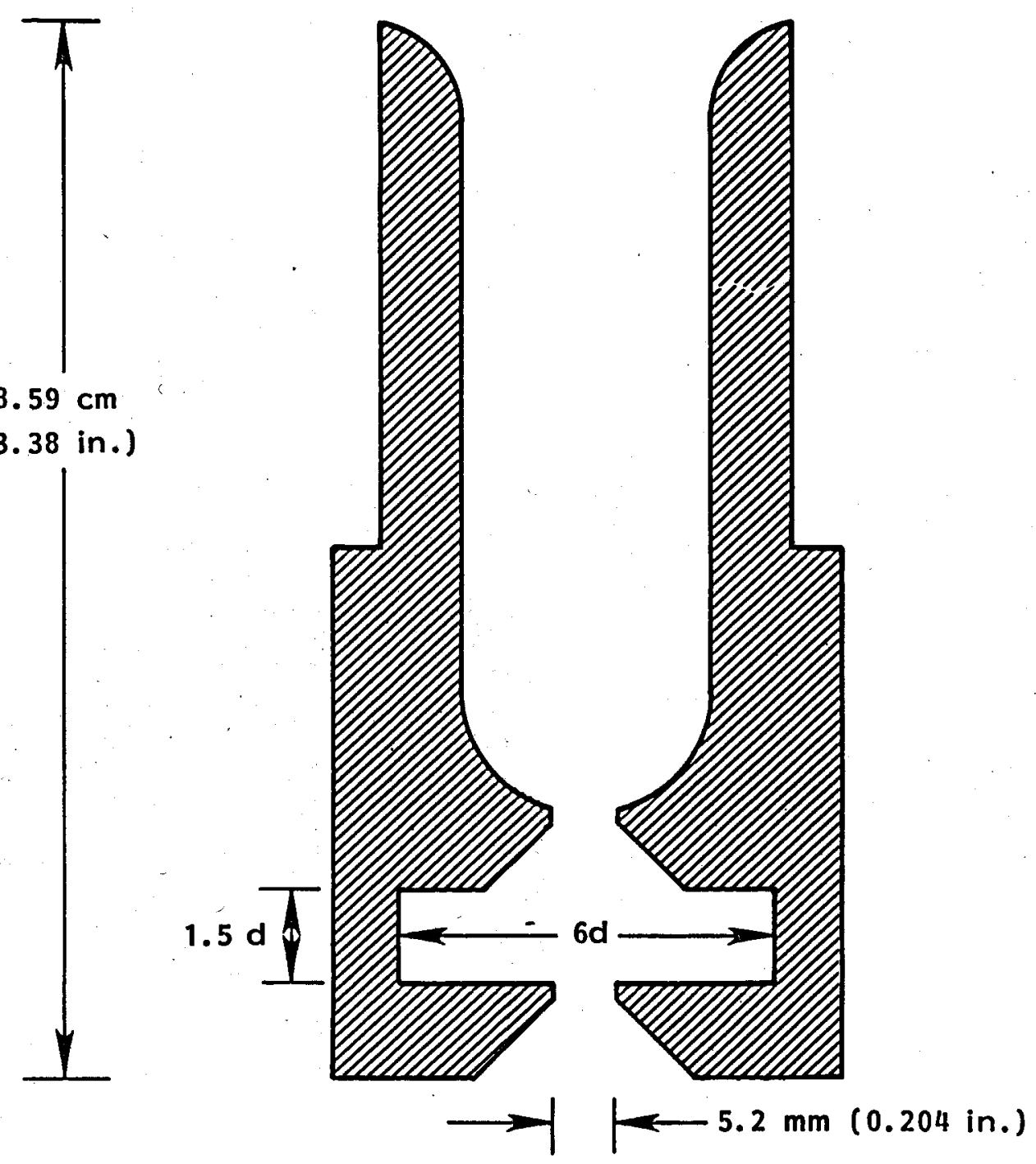

b. PULSER CAVIJET

FIGURE 24 - NOZZLES USED IN GRANITE TESTS AT DRL 


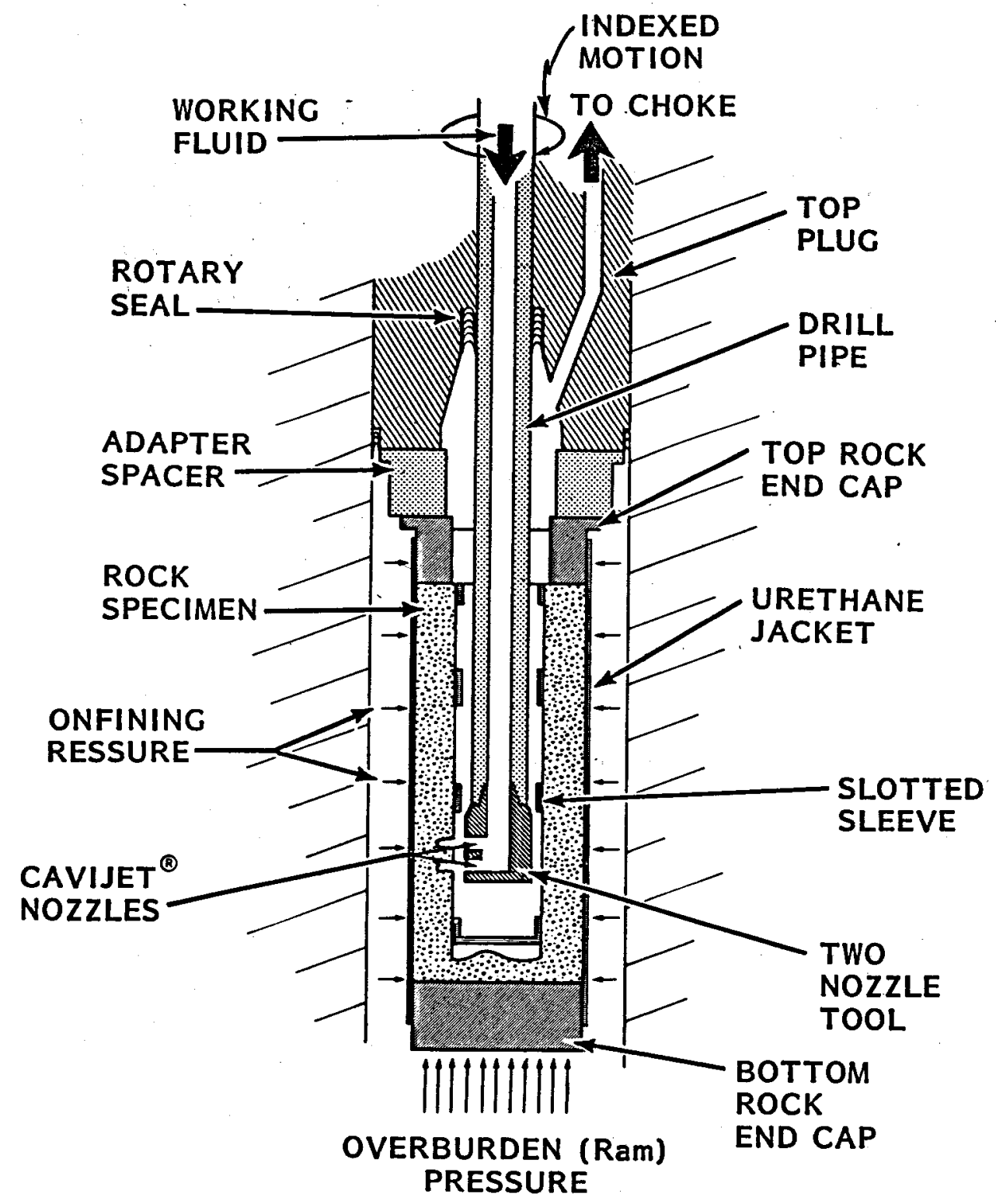

FIGURE 25 - CONFIGURATION FOR STATIONARY NOZZLE TESTS AT DRL 


\begin{abstract}
4.1.1 Test Parameters - The ranges of the various test parameters were:
\end{abstract}

Swivel (pump) pressure

Nozzle pressure drop, $\Delta p$

Ambient ("bore hole") pressure, $\mathrm{p}_{\mathrm{a}}$

Cavitation number $\left(p_{a} / \Delta p\right)$

Stand-off
: 18.6 to $75.8 \mathrm{MPa}$

$$
(2,700 \text { to } 11,000 \mathrm{psi})
$$

: 9.0 to $51.3 \mathrm{MPa}$

$$
\text { (1,300 to } 7,440 \text { psi) }
$$

$: 0.8$ to $51.2 \mathrm{MPa}$

(122 to 7,420 psi)

$: 0.02$ to 5.58

: $21 \mathrm{~mm}(13 / 16$ in.)

It should be emphasized that these ranges of parameters represent the extremal values achieved during the entire test series, and that the maximum values of $\Delta p$ and $p_{a}$ were not utilized during any single.test run. The maximum swivel pressure. which could be used was found to be only about $75.8 \mathrm{MPa}(11,000$ psi), despite the rated pump capacity of $103.4 \mathrm{MPa}(15,000 \mathrm{psi})$. Therefore, since: $p_{\text {swivel }}=\Delta p+p_{a}$, we were precluded from running with any combinations of $\Delta p$ and $p_{a}$ which exceeded the system limit of $75.8 \mathrm{MPa}(11,000 \mathrm{psi})$. This pressure limit on the DRL system during our tests was found to be caused by pipeexpansion at a connection between the aluminum segment of drill pipe (which served as the load cell for sensing torques and bit weights) and the mating steel drill pipe segment. Twice, while we were attempting to exceed $75.8 \mathrm{MPa}(11,000 \mathrm{psi})$, this connection began to leak, requiring a shutdown and replacement of the 0-ring. It was felt that this problem of expansion of the aluminum pipe could probably be dealt with in any future high pressure efforts by substituting a steel drill pipe segment. Time restraints precluded attempting this solution during that test series. 
4.1.2 Nozzle Wear - Another major problem, which was ultimately minimized but not entirely eliminated, was erosion of nozzle orifices by suspended rock particles in the water used for these tests. Since water was selected as the working fluid for these runs, to provide direct comparisons with the more extensive tests to be conducted at HYDRONAUTICS, and because of the long lead time required to fabricate carbide nozzles, all of the nozzles used in these tests were fabricated from stainless steel. During a first test run, the two nozzles were eroded to about twice their original combined area after less than onehalf hour of attempts to achieve the desired test pressures and flows. Thus, it was necessary to repair these two nozzles, by welding over the damaged orifices, and then remachining to approximate the original configurations. These repaired nozzles were subsequently used in the series of runs made on the second specimen of Sierra white granite.

To reduce this nozzle erosion problem, the entire system was thoroughly rinsed with fresh "city" water. Outside water was then used during the actual testing on a once-through basis, instead of being recirculated in the usual fashion. Although this greatly reduced nozzle wear, it was of course not possible to remove all of the particles of rock from the complex DRL piping and pump system. Thus, measurable wear, which was factored into the final analyses of the test results, did occur to all four nozzles.

\subsubsection{Specimen Calibrations - After completion of the} tests at DRL, the two cylindrical specimens of Sierra white granite were each sawn into six pieces for shipment to HYDRONAUTICS. After careful measurements were made of the holes that were eroded at DRL, a segment was selected from each specimen for calibration runs to examine their relative 
erodibility. These runs were made with a 2.72 (0.107 in.) diameter plain CAVIJET nozzle at $\Delta \mathrm{p}=36.9 \pm 0.3 \mathrm{MPa}(5350 \pm 50$ psi). The specimens were submerged in water at atmospheric pressure, and. the standoff was $12.7 \mathrm{~mm}(0.5 \mathrm{in.})$. The calibration holes were all made with a 5-second exposure to the jet, and were made in both the convex and concave sides of the granite specimens.

In many cases, the calibration holes were damaged by cracks that propagated from a hole made later in the series of runs. In these cases, hole depth could still be measured when the rock fragments were pieced back together, but volume information was lost. Most of the holes, however, remained intact.

In repetitive testing, the 5 second runs produced average hole depths typically about 17 to $18 \mathrm{~mm}$ or $\dot{\mathrm{h}}=3.5 \mathrm{~mm} / \mathrm{s} *$. The scatter was about \pm 1 to $\pm 5 \mathrm{~mm}$ around the average depths, except for one set of runs, where the results were: $32 \pm 8 \mathrm{~mm}$. This set seemed to be anomalous; suggesting a particular region of relatively easier-to-erode rock. Volume removals were typically about $2 \mathrm{~cm}^{3}$, with scatter of about \pm 0.4 to $\pm 0.6 \mathrm{~cm}^{3}$. There was no significant difference in the erosion response between the two specimens used in the DRL tests. Thus, in the following discussion of test results it will be valid to make comparisons, not only between tests run on each specimen, but also amongst the various tests on either rock specimen.

Flow calibrations were also run on the four CAVIJET nozzles used in the DRL tests. Although the nozzles were each slightly eroded in the course of the testing at DRL, these calibrations served the purpose of allowing the total flow measured during the DRL tests to be correctly partitioned between the two

* $\dot{h}$ is depth penetration rate, $\mathrm{dh} / \mathrm{dt}$. 
nozzles in each test series. A computer program, originally developed during the earlier phase of this program, was modified and updated for the analysis of the DRL test results.

4.1.4 Erosion Rates - Measured values of eroded hole depth were divided by the corresponding exposure time to find depth penetration rates, $\dot{h}$. Nozzle pressure drops of at least 27.6 $\mathrm{MPa}(4,000 \mathrm{psi})$ were required to create significant erosion in these Sierra white granite specimens. Exposure times of up to five minutes were required to obtain measurable depths for runs at the lower pressures of 10.3 and $17.2 \mathrm{MPa}$ (1,500 and 2,500 psi).

Penetration rates for all four nozzles are compared in Figures $26, \Delta \mathrm{p}=27.6 \mathrm{MPa}$ ( $4 \mathrm{ksi}$ ) and $27, \Delta \mathrm{p}=34.5 \mathrm{MPa}$ (5 ksi) as a function of cavitation number, $\sigma=\mathrm{p}_{\mathrm{a}} / \Delta \mathrm{p}$. The pair of plain CAVIJET cavitating jet nozzles $(2.6 \mathrm{~mm}(0.101 \mathrm{in.})$ and $7.1 \mathrm{~mm}$ ( $0.281 \mathrm{in.})$ orifice diameters) produced substantially lower hole cutting rates compared to the other pair of $5.2 \mathrm{~mm}$ (0.204 in.) plain and PULSER types of CAVIJET cavitating jet nozzles. Enhanced cutting rates for the latter pair of nozzles . was observed over the entire test range of cavitation numbers (from about 0.1 to 1.6 ) which were used at the higher $\Delta p$ 's.

During runs with the $5.2 \mathrm{~mm}$ nozzles, high frequency "chirping" could be heard to emanate from the Well Bore Simulator. A strain gage transducer in the feed pipe transmitted low level signals with discrete frequencies that ranged from 10 to $25 \mathrm{kHz}$. Unlike the case with these modulated CAVIJETS, erosion rates for the plain pair of nozzles decreased monitonically with increasing cavitation number. In the range, $0.2<\sigma<1.6, \dot{h}$ was inversely proportional to $\sigma$ for the $7.1 \mathrm{~mm}$ CAVIJET.

Significant erosion for the smaller $2.6 \mathrm{~mm}$ nozzle occurred 


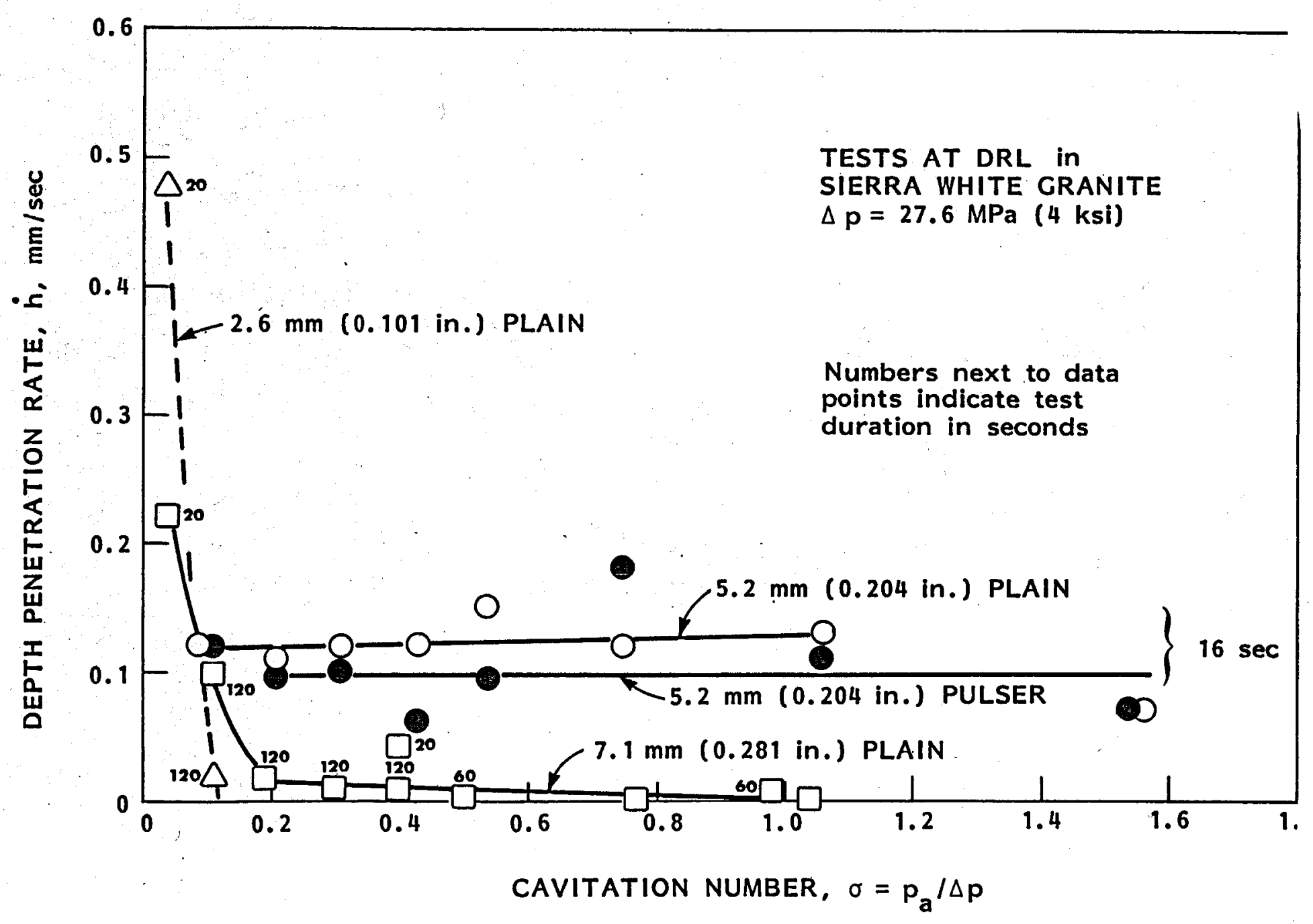

FIGURE 26 - EROSION RATES FOR $\Delta p=27.6 \mathrm{MPa}$ (4 ksi) 


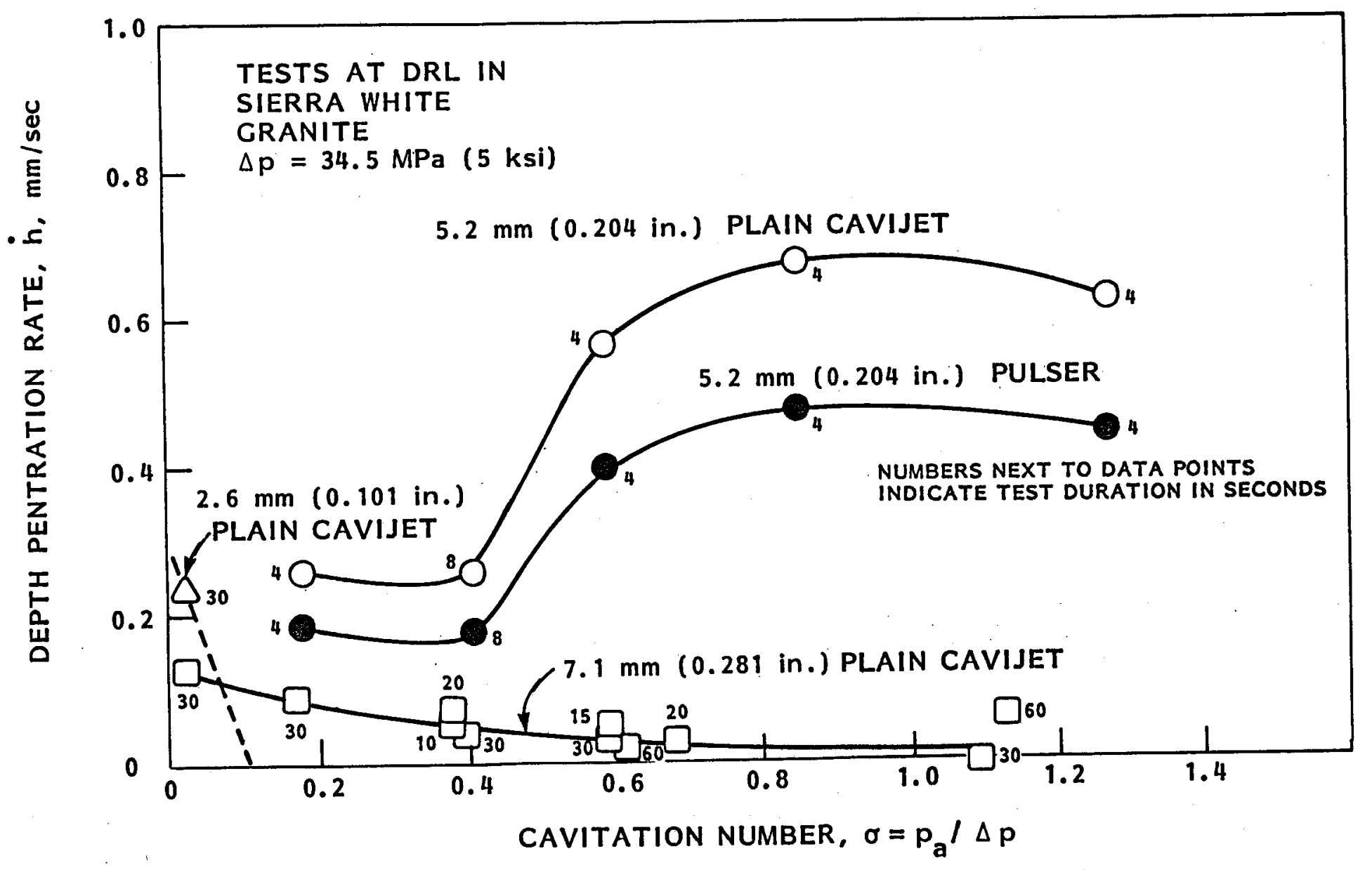

FIGURE 27 - EROSION RATES FOR $\Delta p=34.5 \mathrm{MPa}$ ( $5 \mathrm{ksi}$ ) 
only for the smallest test value of $\sigma=0.04$; at this condition it outperformed the larger $7.1 \mathrm{~mm}$ nozzle. We can attribute this effect to differences in relative standoff, $x / d=8$ and 2.9 for the smaller and larger nozzle, respectively.

A significant finding from these data is that the plain 5.2 CAVIJET outperformed the adjoining PULSER CAVIJET by a factor of about 25 percent. The probable explanation for this behavior is that the plain nozzle was excited by pressure fluctuations created by the resonant PULSER nozzle. The cavitating vortex rings emanating from the plain nozzle could have been better defined than those produced by the double orifice and, therefore, more erosive.

The effect of nozzle pressure on the erosion rate for Sierra white granite is shown in Figure 28. Data for the plain $5.2 \mathrm{~mm}$ and $7.1 \mathrm{~mm}$ nozzles are compared here. The erosion rate with the larger nozzle is seen to increase with $\Delta \mathrm{p}$ to about the ninth power, $\left(h \propto \mathrm{p}^{8.8}\right.$ ). For nozzle pressure between 20.7 and 34.5 $\mathrm{MPa}$ ( 3 and $5 \mathrm{ksi}$ ), the excited $5.2 \mathrm{~mm}$ jet was nominally an order of magnitude more erosive than the larger plain CAVIJET. At higher pressures the data for the two nozzles appear to merge. The amplitude of the PULSER CAVIJET modulations could have been reduced here, and thus the excitation effect on the $5.2 \mathrm{~mm}$ nozzle was lost.

\subsection{Tests on Sedimentary Rocks}

\subsubsection{Calibration of sandstone specimens - Eighty}

$15.2 \mathrm{~cm}(6$ in.) cubes of Berea sandstone were obtained for rock erosion tests in the HYDRONALTICS High Pressure Cell (HPC). This rock had been selected for the preponderance of runs because significant amounts of erosion can be created at rather low nozzle pressure, e.g., 6.9 MPa (1,000 psi). This permitted tests up to cavitation numbers of one in the HPC while using our 


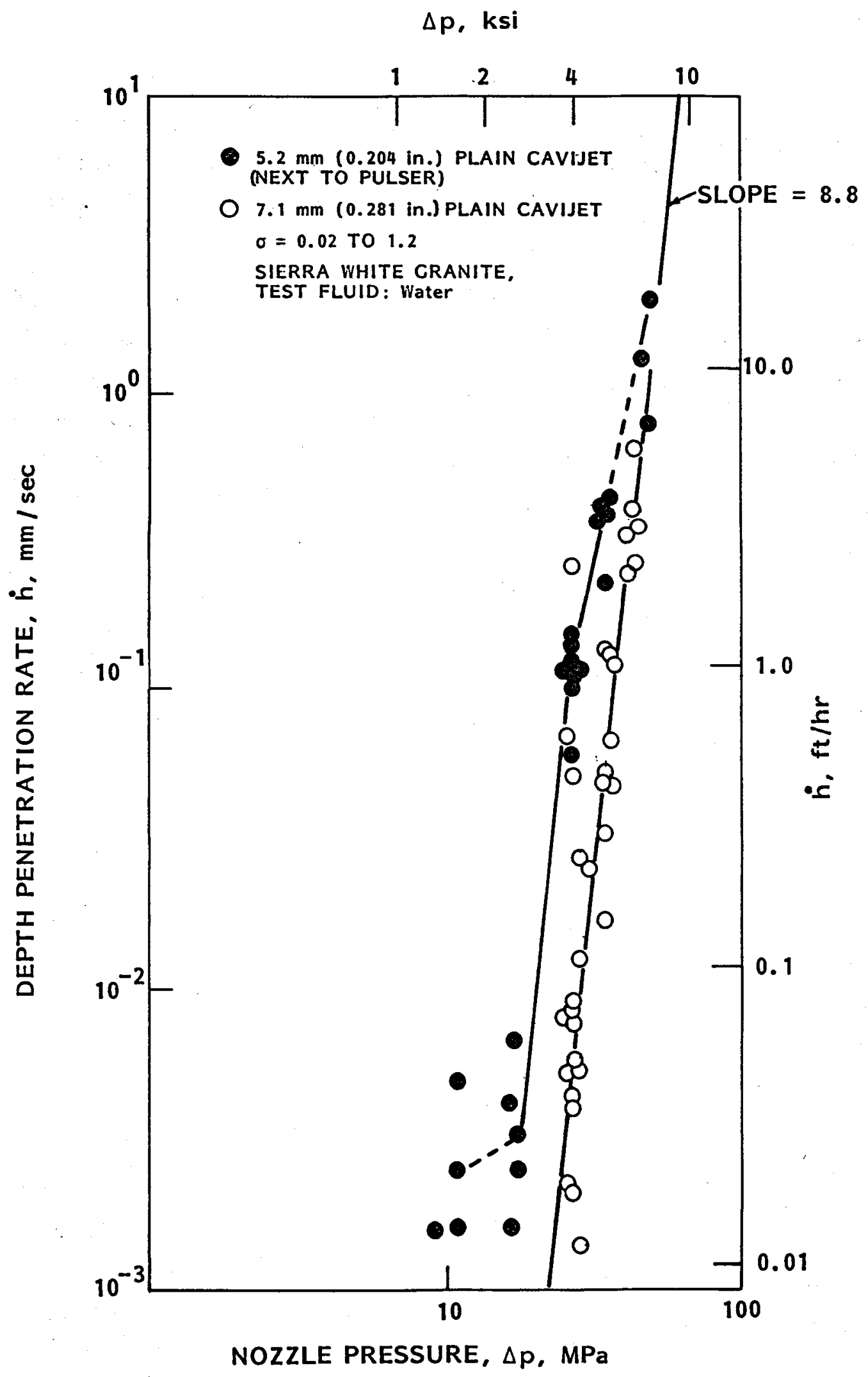

FIGURE 28 - EFFECT OF NOZZLE PRESSURE ON EROSION RATE 
13.8 $\mathrm{MPa}(2,000 \mathrm{psi})$ pump. Initial stationary rock cutting tests, using these Berea sandstone specimens, indicated that the inherent variability of this rock type was going to cause difficulties in performing meaningful comparative testing. From prior testing, it had been established that holes should only be made with the jet normal to the bedding plane. This meant that only two faces of a cube could be subjected to erosion tests.

Some information on specimen uniformity was developed from repetitive tests on two sandstone specimens. Four holes were made with each of two different $2.2 \mathrm{~mm}$ ( 0.086 in.) diameter nozzles in the two faces. Hole depth and volume were measured after $2,4,6,9$, and 12 seconds of jet drilling (submerged, with $\sigma=0.015$ and $6.9 \mathrm{MPa}$ (1,000 psig) driving pressure). One sample was noted as being lighter in color and was observed to be more porous.

Calibration slots (as discussed below) were used to account for differences in the erosion intensity of the two cavitating nozzles, since one was a resonant design. The variations in depth and volume which could therefore be attributed to specimen non-uniformity and test repeatability are summarized below (for 9-second drilling).

\begin{tabular}{|l|c|c|}
\cline { 2 - 3 } \multicolumn{1}{c|}{} & Dark Specimen & Light Specimen \\
\hline $\begin{array}{l}\text { Mean Volume, } \mathrm{cm}^{3} \\
\text { Standard deviation, percent }\end{array}$ & 7.2 & 10.6 \\
\hline $\begin{array}{l}\text { Mean Depth, mm } \\
\text { Standard deviation, percent }\end{array}$ & $14 \dot{5}^{2}$ & $17 \mathbf{1}^{3}$ \\
\hline
\end{tabular}

The holes made in the dark specimen were more uniform than those in the light specimen, but there was a significantly greater difference in holes between the two specimens than was shown for holes within each specimen, particularly with regard to volume removed; the volume removal rate was nearly 50 percent 
greater in the light colored specimen,

These large variations in the erosivity of the Berea sandstone caused us to stop and reassess the decision to use this rock type for nozzle evaluations. Therefore, we examined alternative approaches which included tests on plastics, soft metals such as annealed, pure aluminum or lead, or possible development of screening methods which involve changes in the conductivity of a specimen when a protective coating or oxide coating is removed.

Examination of the erosion results from the various alternative approaches showed that the required brittle, rock-like behavior was not being simulated. We therefore returned to the original concept of using Berea sandstone as our test material. To account for variations, both amongst specimens as well as at different locations on the same $15.2 \mathrm{-cm}(6-\mathrm{in}$.$) cube of rock, we developed$ the "calibration" scheme discussed below to account for these intrinsic variations in the erodibility of this sandstone. Even with calibration, however, the within-specimen variations were often not accounted for completely. Thus, replicative tests were made, when necessary, to further reduce confidence intervals.

The initial concept for this calibration involved using a standard nozzle, at standard conditions of pressure and translation velocity, to cut one or more calibration slots in each face of any specimen used in HPC nozzle performance evaluations. We felt that this approach would minimize the apparent scatter, and permit meaningful comparisons amongst nozzle types. To establish this procedure a comparative translation erosion test series was run with a plain CAVIJET and a PULSER-FED CAVIJET nozzle, both having the same exit orifice diameter, $d=5$ ( 0.196 in.). The slot depths from both the test cuts and 


\section{$-44-$}

calibration cuts were carefully analyzed.

These analyses indicated that the cause of variability between tests could only be partially attributed to scatter in the intrinsic erodibility between rock specimens. Indeed, while "calibrating" several specimens at the same time in our large, atmospheric pressure test chamber, the standard deviation of the variation in calibration slot depth between specimens was only about 5 percent of the mean depth. However, the variability between depths of cuts made on one specimen at the same test conditions, but when run at different times, was about 10 percent (standard deviation of mean depth).

This suggested that the rock specimens used in this test sequence were themselves more uniform than our ability to control the parameters to achieve a calibration cut. These parameters include translation speed and nozzle pressure, particularly the latter. For example, if jet erosivity follows a 4th power law (with respect to nozzle pressure), a 3 percent error in setting $\Delta \mathrm{p}$ would contribute a 13 percent error to slot depth $\left(1.03^{4}=\right.$ 1.13).

Raw data for mean slot depths that were cut in the calibrated specimens had differences averaging 11 percent between pairs of independent runs at the same test condition. When correction factors (ranging from 1.0 to 1.08) derived from the calibration cuts were applied to the data, the differences actually increased to an average of 12 percent between pairs. At best, assuming stochastic independence, an 11 percent standard deviation should only be improved to 10 percent when the correction only amounts to 5 percent $\left(0.11^{2}-0.05^{2}=0.10^{2}\right)$. 
Since calibration by standard nozzle slot cutting failed to reduce data scatter significantly, we adopted a strategy for comparative tests wherein replications were made and pairs of comparative runs were cut on two halves of the same specimen face. Finally, we found that multiple passes at a higher translation velocity seemed to minimize (or smooth out) the undulations created by using a single pass at a lower translation velocity. Multiple passes are, of course, more replicative of the actual action of a drill bit.

4.2.2 Test Results - We carried out several tests to compare the rock cutting ability of structured versus unstructured cavitating jets. Figure 29 shows the dimensions of an organpipe configuration used to feed either a standard SMITH Tool roller cone nozzle shape or a CAVIJET nozzle, each with $d=$ $6.4 \mathrm{~mm}(0.25 \mathrm{in.})$. These nozzles were tested in the High Pressure Cell at various nozzle pressure drops and ambient pressures.

A typical comparison is shown in Figure 30 between the CAVIJET self-resonating nozzle and the conventional SMITH nozzle. Seen is a top view of one face of a $15.2 \mathrm{~cm}$ ( 6 in.) cube specimen of Indiana limestone. To allow a half-circle cut to be made by each nozzle, one half of the rock face was protected by a metal plate. The nozzle was then changed, the plate shifted to protect the other half of the cube-face, and the same conditions were run again. In this way; variations due to rock properties could be minimized. Under these conditions, the mean slot depth cut by the ORGAN-PIPE CAVIJET was 4.3 times deeper than that cut by the SMITH nozzle. It should be emphasized that, although the same organ pipe inlet was used with both nozzle shapes, the SMITH nozzle did not provide the feed back mechanism that is necessary to sustain passive modulation. 


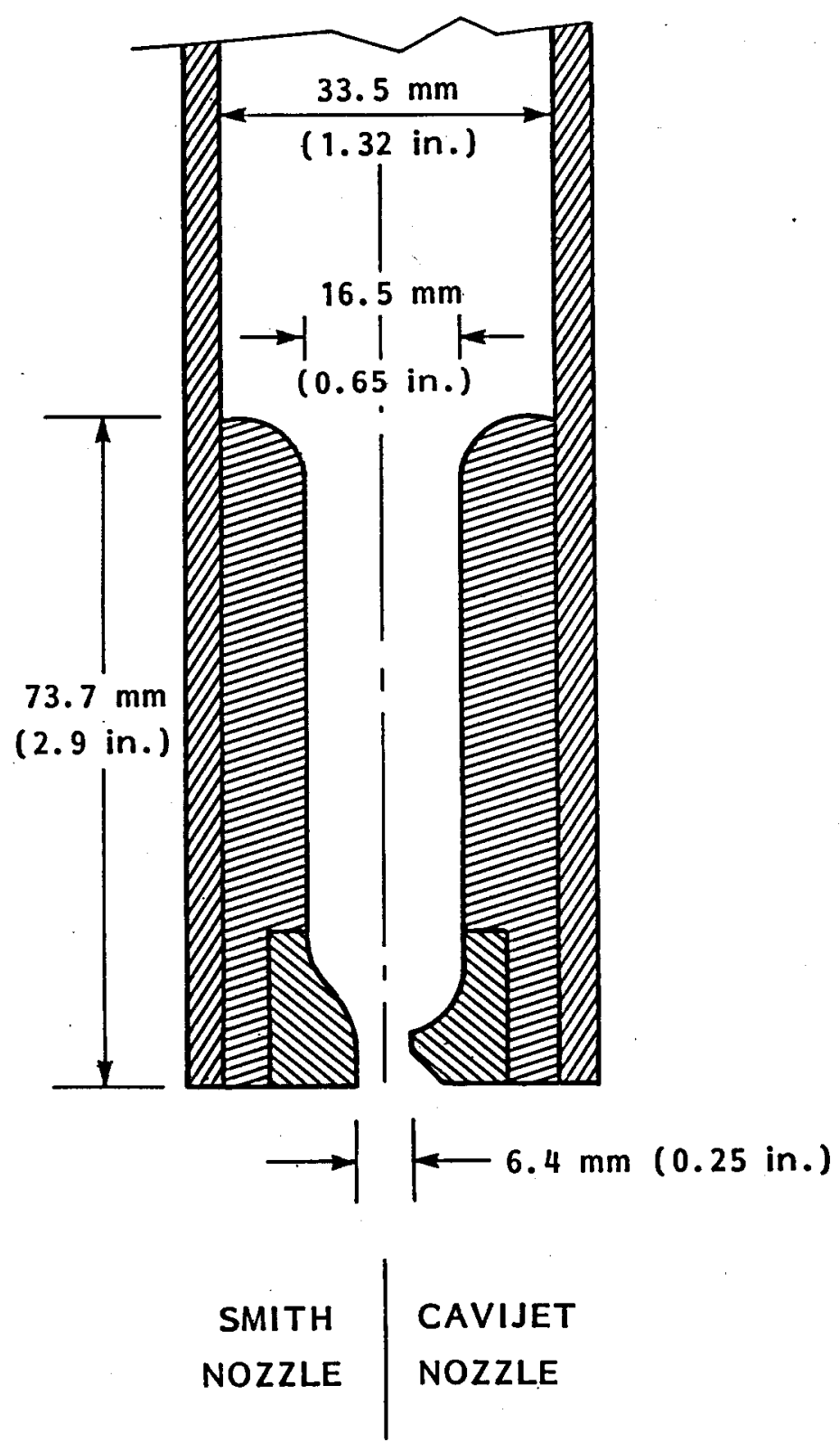

FIGURE 29 - CONFIGURATIONS OF ORGAN-PIPE NOZZLES USED IN ROCK CUTTING TESTS (See Figure 30) 

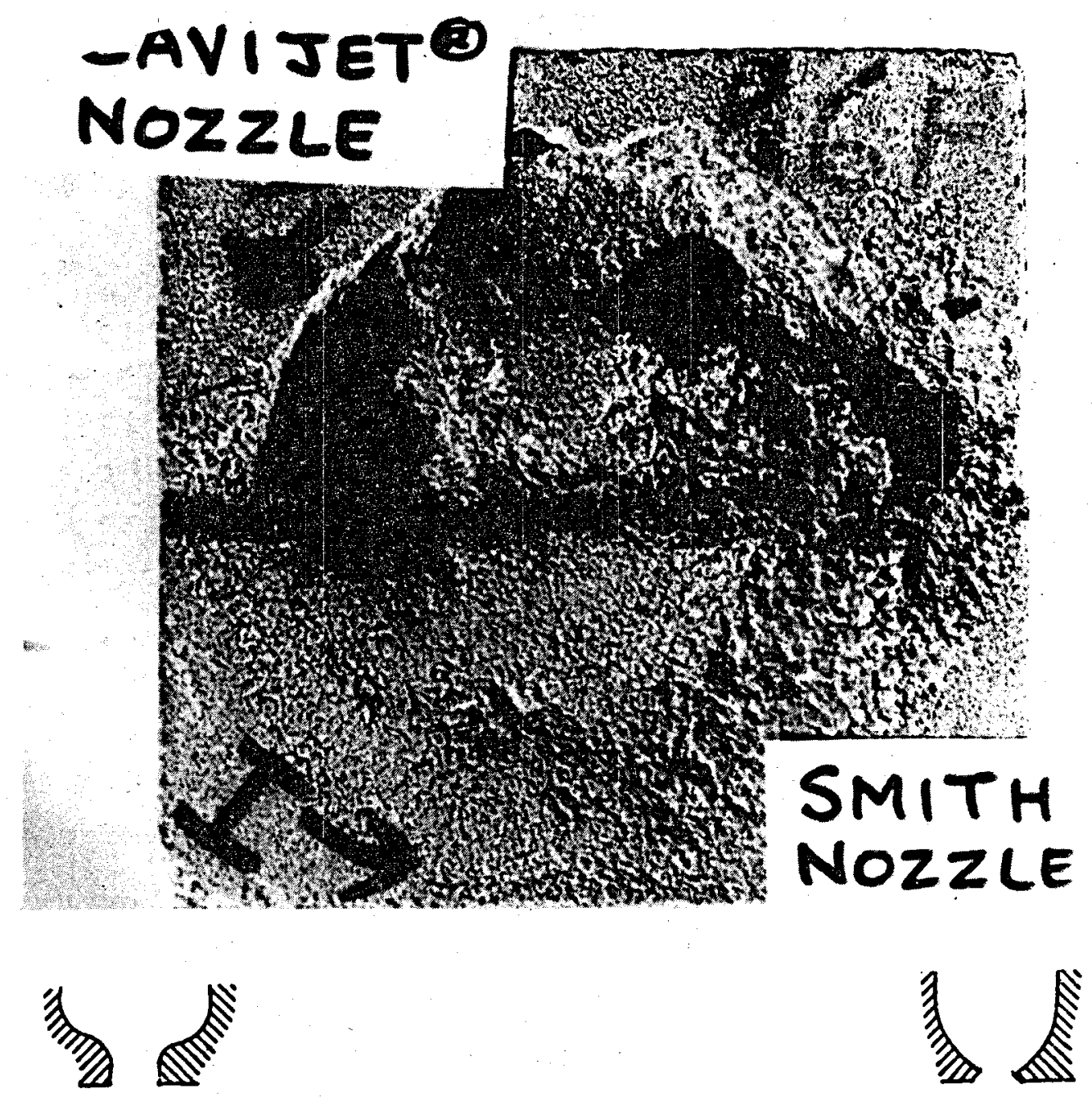

SMITH ORGAN-PIPE CAVIJET $^{\circledR}$

ROCK: Indiana limestone, STANDOFF: $X / d=3$ NOZZLE PRESSURE: $\Delta p=10.3 \mathrm{MPa}(1,500 \mathrm{psi})$ AMBIENT PRESSURE: $p_{a}=0.83 \mathrm{MPa}(120 \mathrm{psi})$ CAVITATION NUMBER: $\sigma=0.08$ TEST MODE: Single pass in HPC TRANSLATION VELOCITY: $v=6.4 \mathrm{~mm} / \mathrm{s}(0.25 \mathrm{in.} / \mathrm{s})$ NOZZLE ORIFICE DIAMETER: $d=6.4 \mathrm{~mm}(0.25 \mathrm{in.})$ MEAN SLOT DEPTHS: $\begin{aligned} & \text { SMITH } \\ & \text { CAVIJET: }\end{aligned} \begin{array}{r}4.3 \mathrm{~mm}(0.17 \mathrm{in.)} \\ 18.3 \mathrm{~mm}(0.72 \mathrm{in.)}\end{array}$ CAVITATING JET AND A CONVENTIONAL JET 
Since a major goal of our studies has been to design selfexcited nozzle systems capable of replacing the nozzle systems of current bit designs, it was necessary to compare the rock cutting performance of candidate new designs with typical existing designs. Figure 31 shows a comparison of the geometry of an existing design (SMITH) and an early candidate CAVIJET nozzle system designed to self excite and structure the jet. The model designs shown are half the full scale dimensions. In this case, the feed tube leading to the SMITH nozzle represented the actual geometry of the extended tubes used in $31 \mathrm{~cm}$

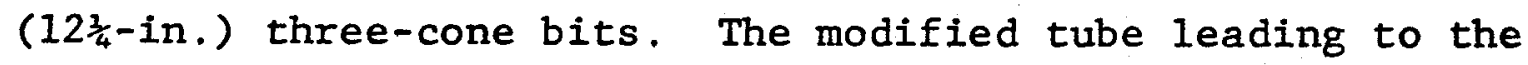
CAVIJET nozzle was an early two-step organ pipe design.

Table 2 presents some rock cutting data for the two nozzles in Figure 31. Except at the very low cavitation number of 0.067 , the ORGAN-PIPE CAVIJET nozzle system cut this limestone to a depth 2 to 2.5 times greater than the standard SMITH system. It is important to note that for the pressure drops of $17.2 \mathrm{MPa}$ $(2,500 \mathrm{psi})$ or less which are currently used in drilling practice, we do not expect the jets to deeply kerf the rock - even with improved nozzle systems under cavitating conditions. However, the more intense excited jets should provide enhanced cleaning of the hole bottom. The rock cutting results presented here are for traversing rates much less than those used in rotary drilling and serve only as a measure of the relative intensity of various designs. 

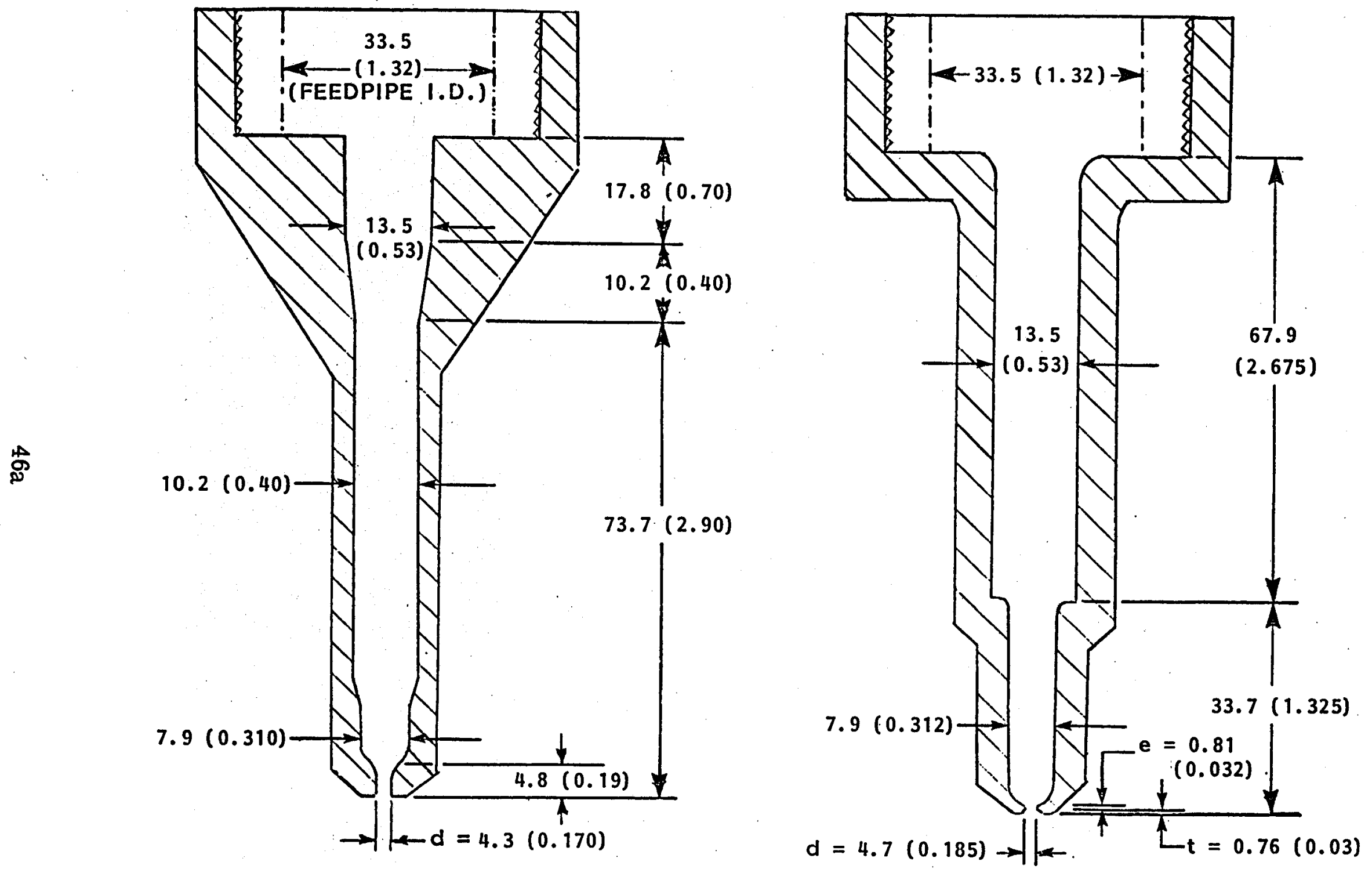

ALL DIMENSIONS ARE mm (in.)

\section{SMITH}

CAVIJET ${ }^{\circledR}$

FIGURE 31 - HALF-SCALE NOZZLES USED IN COMPARATIVE ROCK CUTTING TESTS 
Table 2

SLOT CUTTING COMPARISONS

NOZZLES: Conventional SMITH, $\mathrm{d}=4.3 \mathrm{~mm}$ ( $0.170 \mathrm{in.)}$ ORGAN-PIPE CAVIJET, $d=4.7 \mathrm{~mm}(0.185$ in.) ROCK: Indiana limestone; STANDOFF: $\mathrm{X} / \mathrm{d}=3$ TRANSLATION VELOCITY: $6.4 \mathrm{~mm} / \mathrm{s}(0.25 \mathrm{in./ \textrm {s }})$

\begin{tabular}{|c|c|c|c|}
\hline $\begin{array}{c}\text { Pressure, } \\
\text { Nozzle } \\
\Delta \mathrm{p}, \mathrm{MPa} \text { (psi) }\end{array}$ & $\begin{array}{c}\text { Ambient } \\
\text { Pressure } \\
\mathrm{p}_{\mathrm{a}}, \mathrm{MPa} \text { (psi) }\end{array}$ & $\begin{array}{c}\text { Cavitation } \\
\text { Number, } \\
\sigma\end{array}$ & $\begin{array}{c}\text { Mean Slot } \\
\text { Depth Ratio, } \\
\text { CAVIJET } \\
\text { SMITH }\end{array}$ \\
\hline \multicolumn{5}{|c|}{ SINGLE PASS } \\
\hline $17.4(2,530)$ & $1.2(170)$ & 0.067 & 1.16 \\
$17.2(2,500)$ & $4.1 \quad(600)$ & 0.24 & 2.54 \\
$17.2(2,500)$ & $8.3(1 ; 200)$ & 0.48 & 2.33 \\
\hline \multicolumn{6}{|c|}{ TWENTY-FIVE PASSES } \\
\hline $8.4(1,225)$ & $2.2 \quad(325)$ & 0.27 & 1.90 \\
\hline
\end{tabular}

Note that for these single pass tests, as $\sigma$ is increased the performance of the ORGAN-PIPE CAVIJET improves relative to the SMITH nozzle. The cavitation inception number for this CAVIJET nozzle was more than twice that for the SMITH nozzle. This result is consistently observed, that is, the jet having the higher inception cavitation number is found to be nore erosive than a jet of the same size, operated under identical conditions, but having a lower $\sigma_{i}$.

Comparative test series were run with plain CAVIJETS and two PULSER type modulated jets. Erosion performance of an elementary PULSER design is shown in Figure 32 along with data 
$P_{a}=5.5 \mathrm{MPa}(800 \mathrm{psi})$

$\Delta p=6.9 \mathrm{MPa}(1000 \mathrm{psi})$
10 passes at $7.6 \mathrm{~cm} / \mathrm{s}(3 \mathrm{in.} / \mathrm{s})$

$\sigma=0.8$

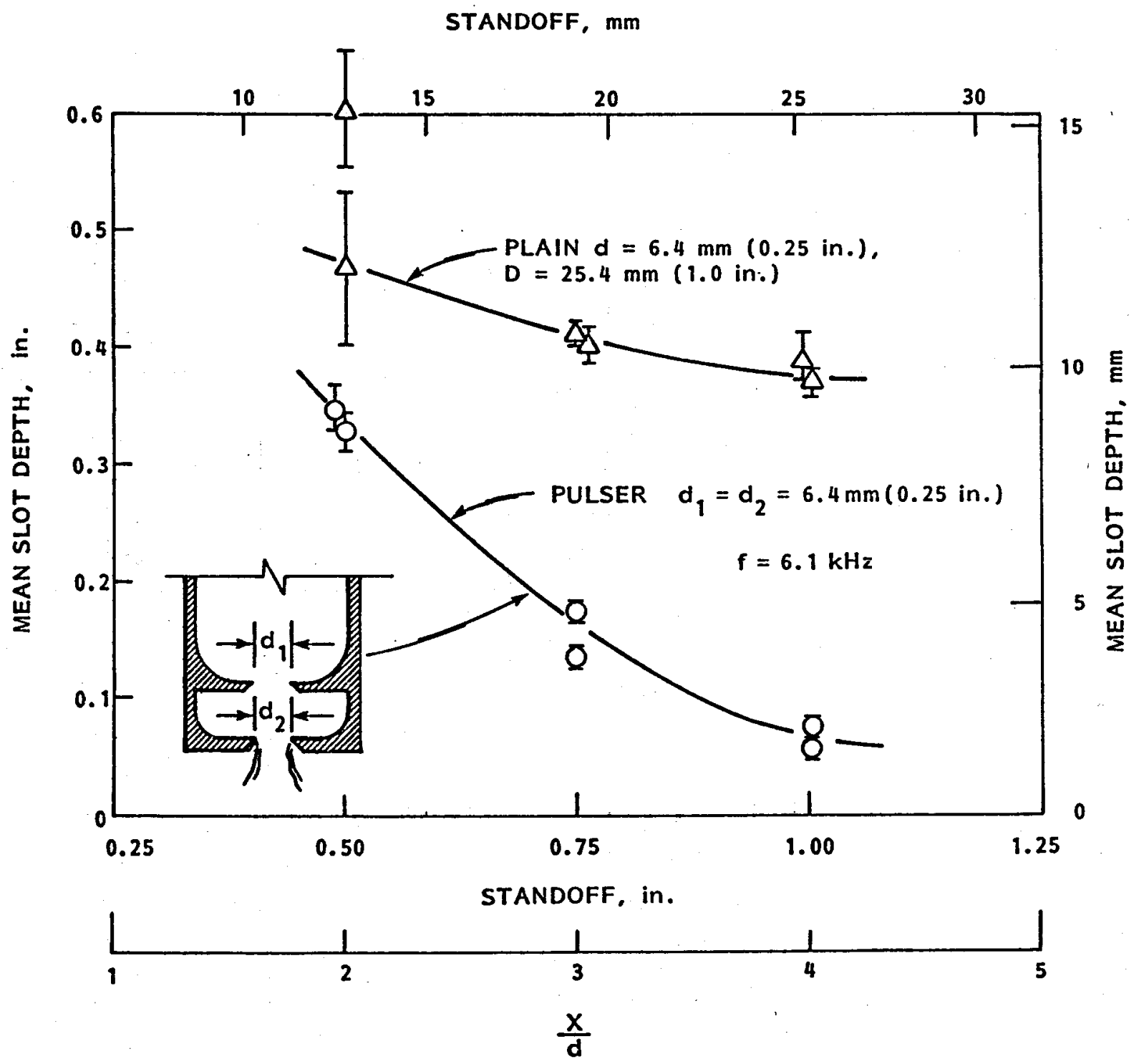

FIGURE 32 - COMPARATIVE EROSIVITY OF A PULSER AND A PLAIN CAVIJET 
for a plain CAVIJET. Error bars on the data points denote the standard deviation of slot depth about the measured mean value. These data depict a rare case wherein the structured jet was less erosive than a steady jet at the same mean nozzle pressure. Performance of a PULSER-FED CAVIJET nozzle is compared to a plain CAVIJET in Figure 33. In this series the supply pressure for the PULSER-FED nozzle was adjusted to account for line losses through the upstream PULSER section so that the mean jet velocity would be comparable to the plain CAVIJET case.

A summary of similar test results is presented in Table 3.

Apparent inconsistencies in the results achieved when using the Berea sandstone to compare a plain CAVIJET and a PULSER CAVIJET nozzle have led us to begin to question how this particular type of rock responds: a) under impulsive loading, and b) under high ambient pressure. Although we have only begun to examine these questions, an initial check of the literature revealed that similar rock cutting tests on Berea sandstone have produced comparable observations.

For instance, rock cutting tests with an interrupted jet, in air, by Nebeker (16), showed improved rates of cutting over a conventional steady jet in a number of rock types including limestones and granite. With Berea sandstone, however, Nebeker reported essentially no difference in erosion between the two jets. In single bit-tooth erosion tests, conducted both statically and dynamically under elevated ambient pressures (with water or with drilling mud as the pressurizing fluid) Maurer (17) observed a number of effects on the erodibility of Berea sandstone and Indiana limestone. It is possible that Maurer's observations can be related to some of the inconsistencies we have experienced. 
STANDOFF, $\mathrm{mm}$

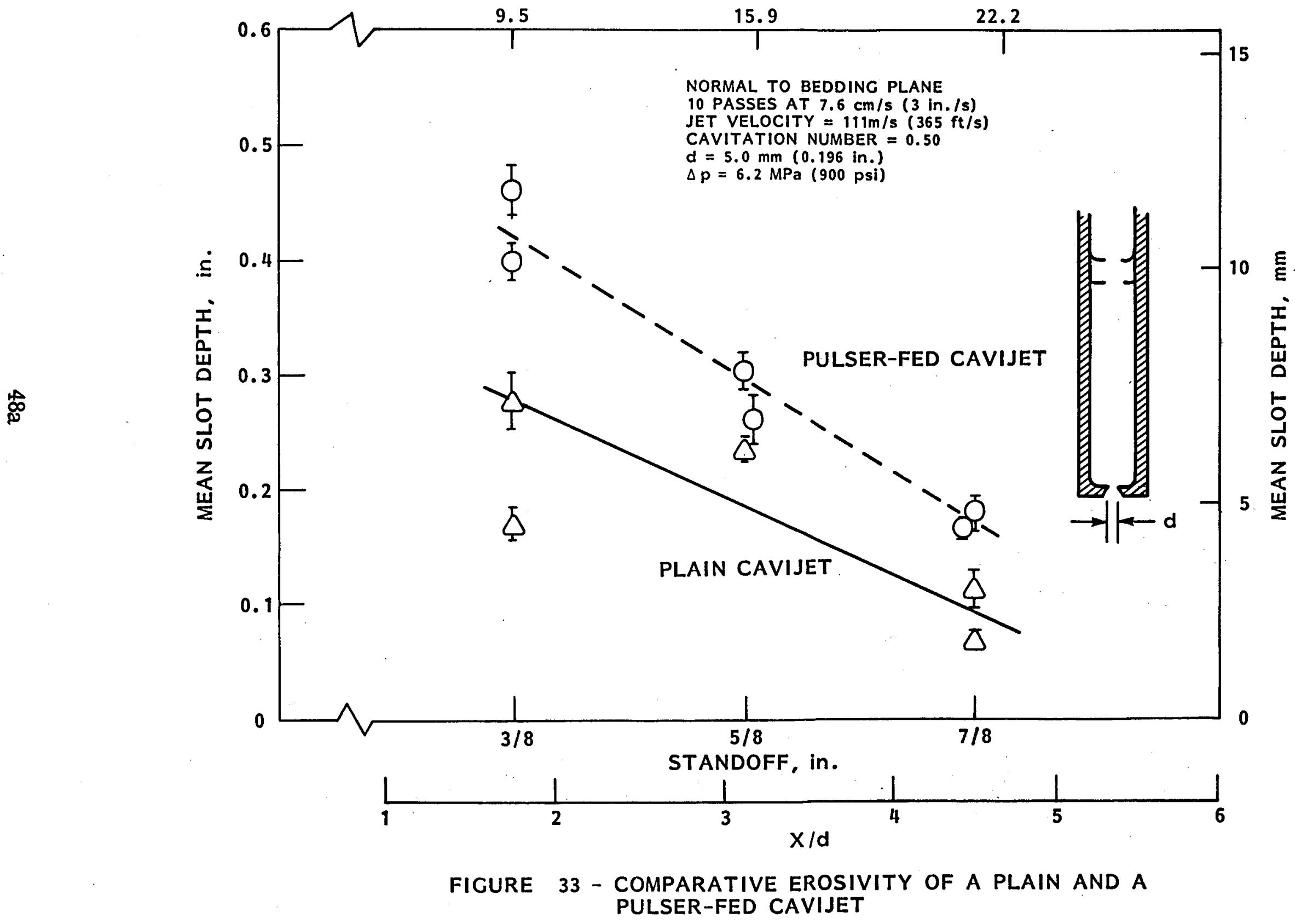


TABLE 3

COMPARISON OF RELATIVE PENETRATION OF ROCK FOR SOME RESONATING NOZZLE TYPES TESTED (RELATIVE TO BASIC CAVIJET

\begin{tabular}{|c|c|c|c|c|c|c|c|}
\hline Nozzle Type & $\begin{array}{l}\text { Nozzle } \\
\text { Diameter, } \mathrm{d} \\
\mathrm{mm} \text { (in.) }\end{array}$ & $\begin{array}{c}\text { Nozzle } \\
\text { Pressure } \\
\text { Drop, } \Delta \mathrm{p} \\
\mathrm{MPa} \text { (psi) }\end{array}$ & $\sigma$ & $\mathrm{x} / \mathrm{d}$ & $\begin{array}{c}\text { Cut } \\
\text { Type }\end{array}$ & Rock & $\frac{h}{h_{0}}, \frac{\dot{h}}{h_{0}}$ \\
\hline PULSER & $6.4(0.250)$ & $6.9(1000)$ & 0.8 & 2.5 & Slot & B. Sandstone & 0.45 \\
\hline PULSER & $6.4(0.250)$ & $6.9(1000)$ & 0.8 & 2.5 & Slot & I. I,imestone & $1.1-1.9$ \\
\hline PULSER & $6.4(0.250)$ & $6.9(1000)$ & 0.8 & 2.0 & Hole & I. Limestone & $2.4-4.5$ \\
\hline PULSER & $5.2(0.204)$ & $34.5(5000)$ & 0.6 & 4.0 & Hole & S. W. Granite & 12.9 \\
\hline PULSER-FED & $5.0(0.196)$ & $6.2(900)$ & 0.5 & 2.5 & Slot & B. Sandstone & 1.5 \\
\hline PULSER-FED & $5.0(0.196)$ & $4.3 \quad(625)$ & 0.1 & 2.5 & Hole & B. Sandstone & 1.4 \\
\hline PULSER-FED & $5.0(0.196)$ & $6.9(1000)$ & 0.1 & 2.5 & Hole & I. Limestone & 1.5 \\
\hline PULSER EXCITED CAVIJET & $5.2(0.204)$ & $34.5(5000)$ & 0.6 & 4.0 & Hole & S. W. Granite & 18.4 \\
\hline
\end{tabular}


Rock type was also discovered to affect the dependence of erosion on several primary variables, in addition to the previously known influence of the exponent used to correlate erosion rate with nozzle pressure, and in the absence of complicating resonant jet effects.

A test series involving three plain CAVIJET nozzles with orifice diameters, $d$, of: $2.6,6.4$, and $7.9 \mathrm{~mm}(0.101,0.250$, and $0.312 \mathrm{in.)}$ was undertaken. The contraction ratio for each nozzle was four as shown in Figure 34. Slot cutting tests were made on Indiana limestone: and Berea sandstone, over a range of standoff distances, $X(X / d=3$ to 5$)$. The normalized data are presented in Figures 35 and 36, respectively. Our analyses of these results suggest that rock properties affect the dependence of slot depth, $h$, on nozzle diameter, $d$, and standoff distance, $x$. Specifically, for the Indiana linestone, these preliminary data could be correlated according to: $\mathrm{hx}^{3 / 2} / \mathrm{d}^{5 / 2}$. However, for the Berea sandstone, the comparable data correlation was achieved with: $\mathrm{hX} / \mathrm{d}^{3 / 2}$. 


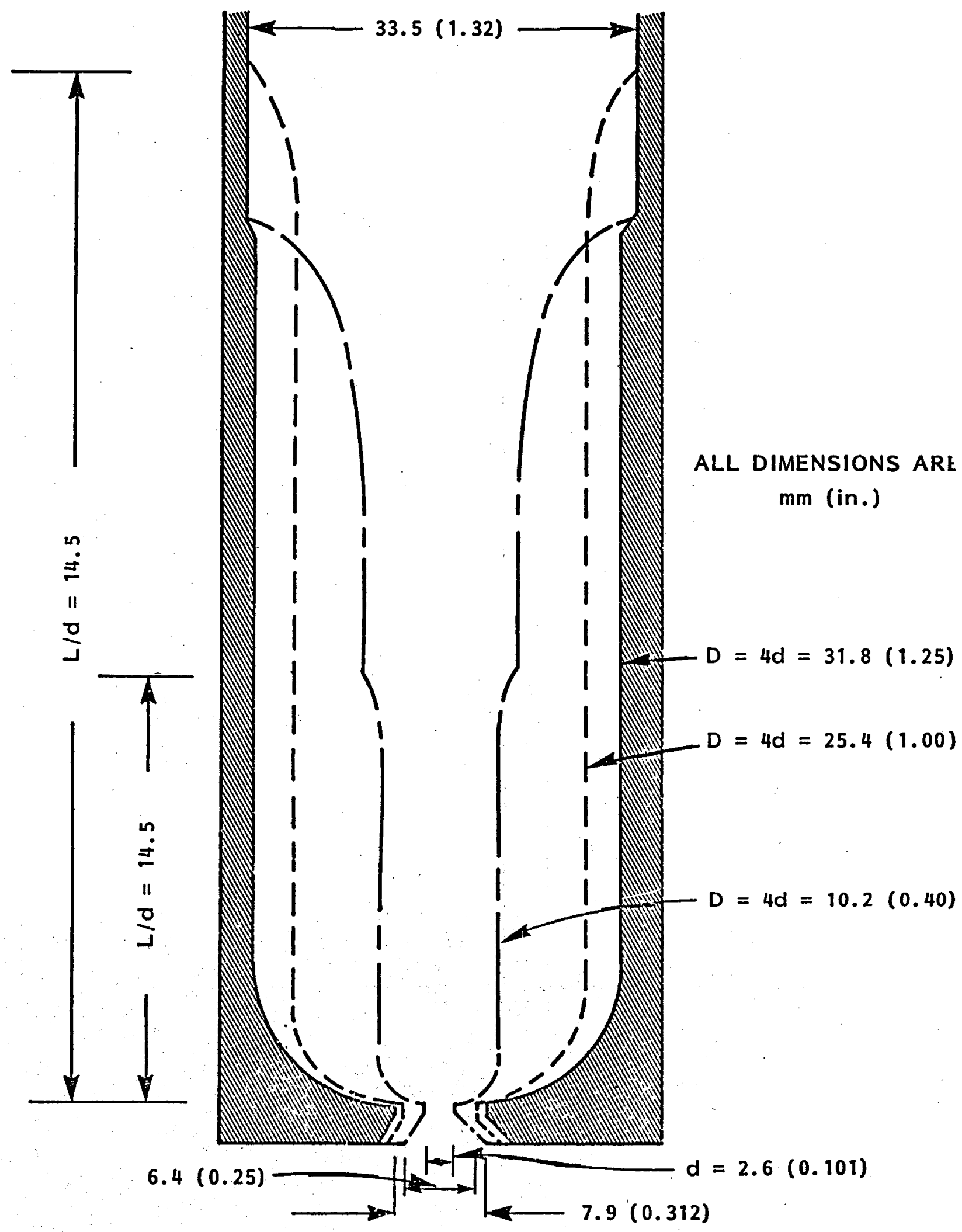

FIGURE 34 - BASIC PLAIN CAVIJET SIZE SERIES 


$$
\begin{array}{ll}
N=60 \text { passes } & \Delta p=10.2 \mathrm{MPa}(1480 \mathrm{psi}) \\
V_{T}=7.6 \mathrm{~cm} / \mathrm{s}(3 \mathrm{in} . / \mathrm{s}) & V_{0}=140 \mathrm{~m} / \mathrm{s}(460 \mathrm{ft} / \mathrm{s}) \\
\sigma=0.135 &
\end{array}
$$

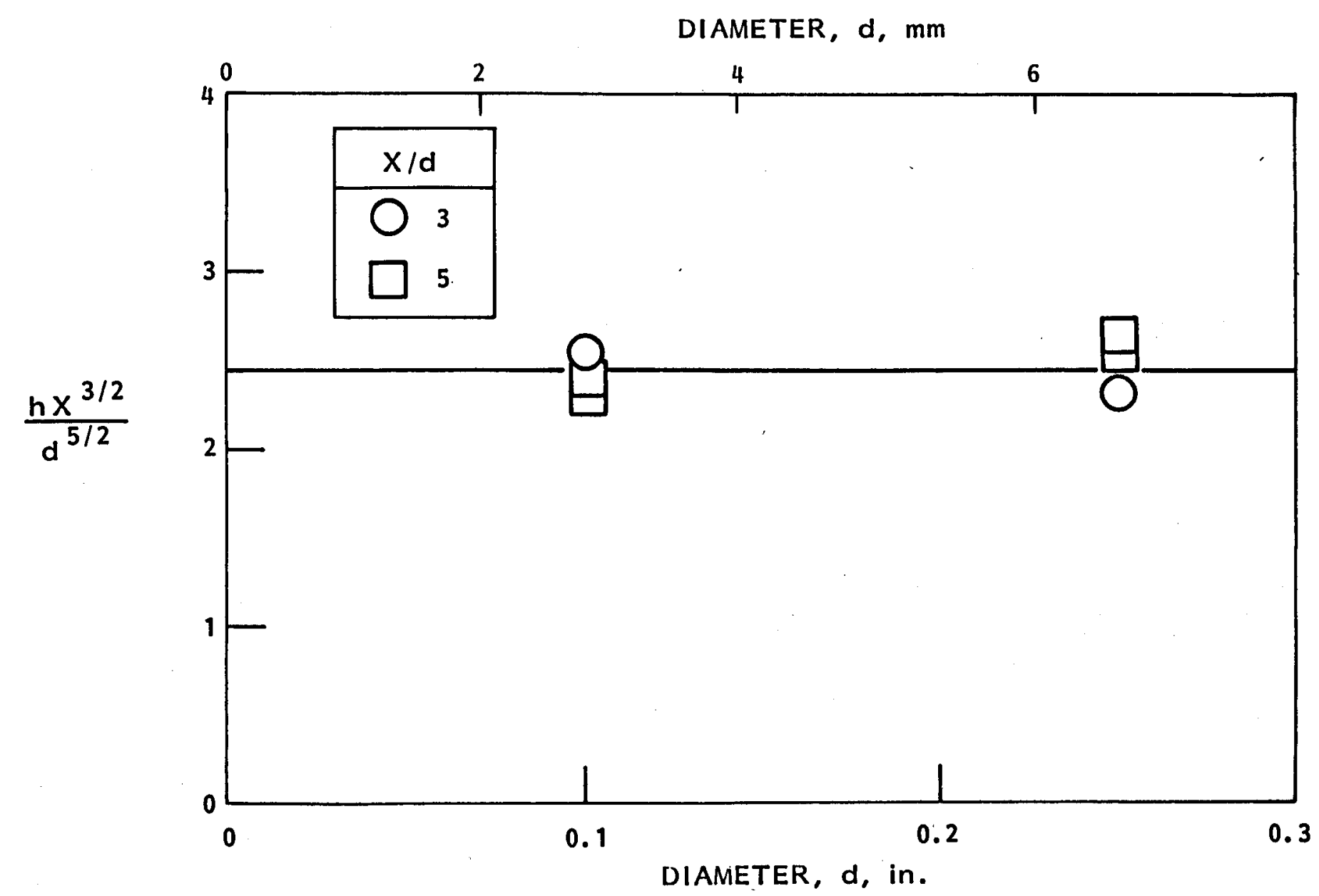

FIGURE 35 - EFFECT OF.SIZE AND STANDOFF ON SLOT CUTTING PENET,RATION FOR PLAIN CAVIJET, INDIANA LIMESTONE 


$$
\begin{array}{ll}
N=6 \text { passes } & \Delta p=6.9 \mathrm{MPa}(1000 \mathrm{psi}) \\
V_{\mathrm{T}}=7.6 \mathrm{~cm} / \mathrm{s}(3 \mathrm{in} . / \mathrm{s}) & V_{0}=117 \mathrm{~m} / \mathrm{s}(385 \mathrm{ft} / \mathrm{s}) \\
\sigma=0.40 &
\end{array}
$$

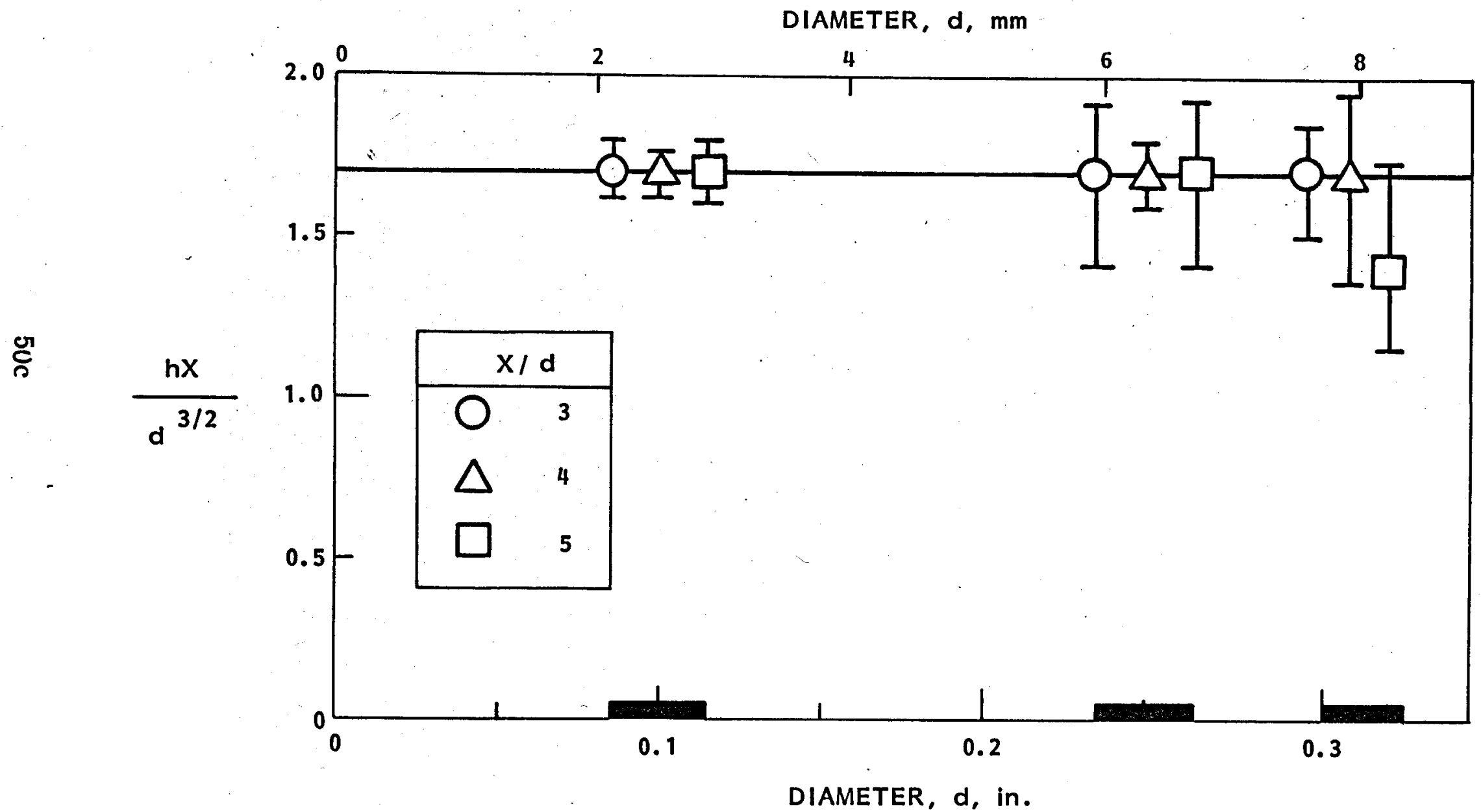

FIGURE 36 - EFFECT OF SIZE AND STANDOFF ON SLOT CUTTING PENETRATION FOR PLAIN CAVIJET, BEREA SANDSTONE 


\subsection{CHIP LIFTING TESTS}

A new low pressure test loop facility was designed, assembled and operated to provide empirical support to the concepts dealing with jet structuring effects on bottom hole cleaning (see Appendices $A$ and $C$ ). Additional information was derived from tests performed with air on large scale STRATOJET (ORGAN PIPE) nozzle configurations. A description of the new facility and test results are presented in this section.

\subsection{Chip Hold-Down Facility}

The bench mounted flow loop contains two pumps, a reservoir. tank, an organ-pipe feed line, and test chamber as pictured in Figure 37. The test chamber was constructed of a clear acrylic cylinder with aluminum end plates that were held in place by means of threaded tie rods (see Figure 38 ). The inside diameter of the chamber was $28 \mathrm{~cm}$ (11 in.).

A porous stainless steel plate bridged the bottom of the cell atop a cylindrical aluminum spacer. This plate supported the various filter screens on which mud beds were established. Also mounted in this plate were four piezoelectric pressure transducers (PCB Piezotronics Model 101A05) that were flush mounted as shown in Figure 39.

A small, $0.32 \mathrm{l} / \mathrm{s}(5 \mathrm{gpm})$ positive displacement pump served to pressurize the test cell, up to $1,1 \mathrm{MPa}$ (165 psia), and supplied flux across the porous metal/mud bed to generate chip hold-down forces. The flux was monitored with a rotameter and controlled with a by-pass valve.

The centrifugal jet supply pump took suction directly from the test cell and discharged through a flexible hose to a short length of plenum pipe. The plenum fed the $61 \mathrm{~cm}$ (24 in.) long 


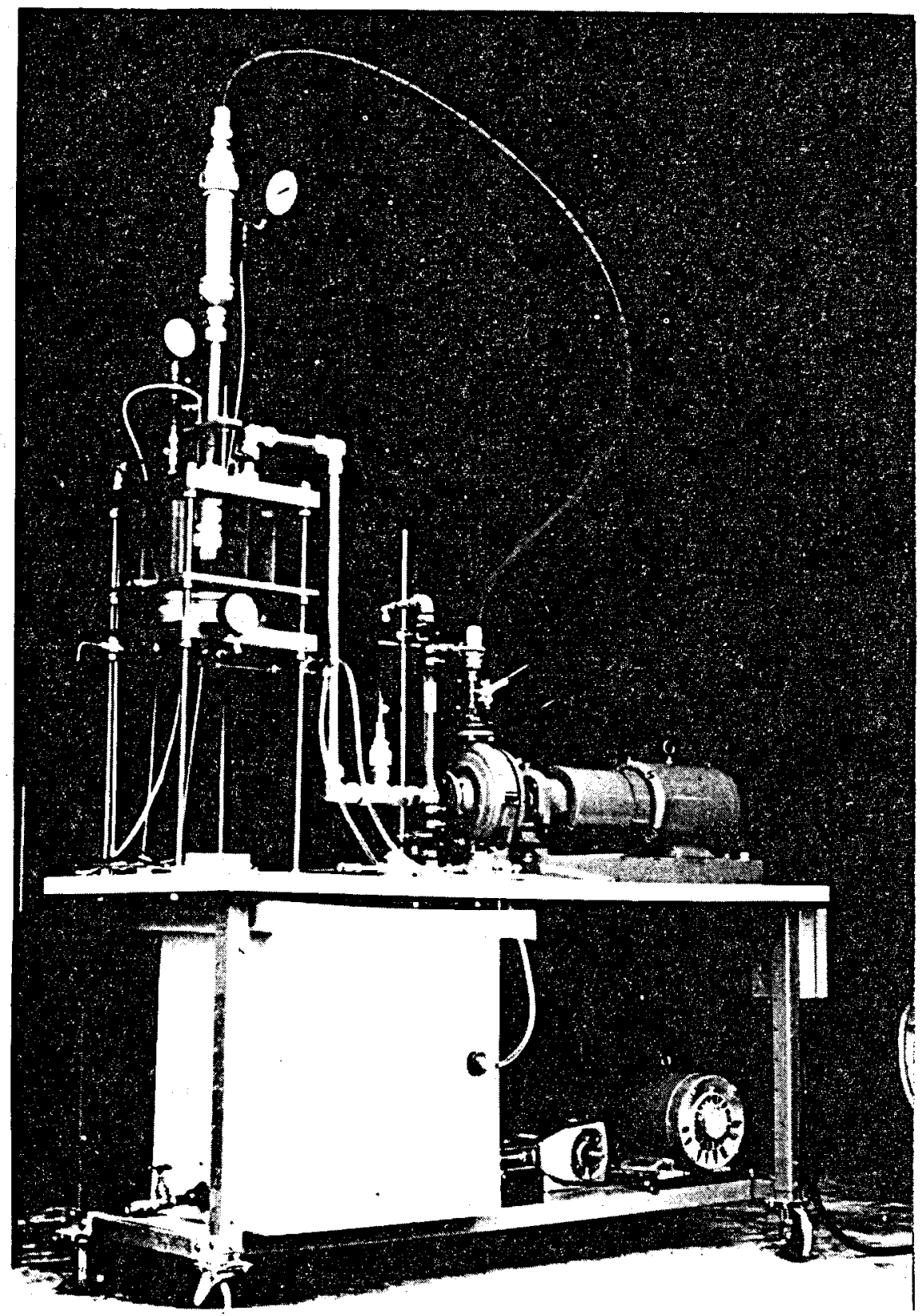

FIGURE 37 - NEW, LOW PRESSURE, CHIP HOLD-DOWN TEST LOOP 


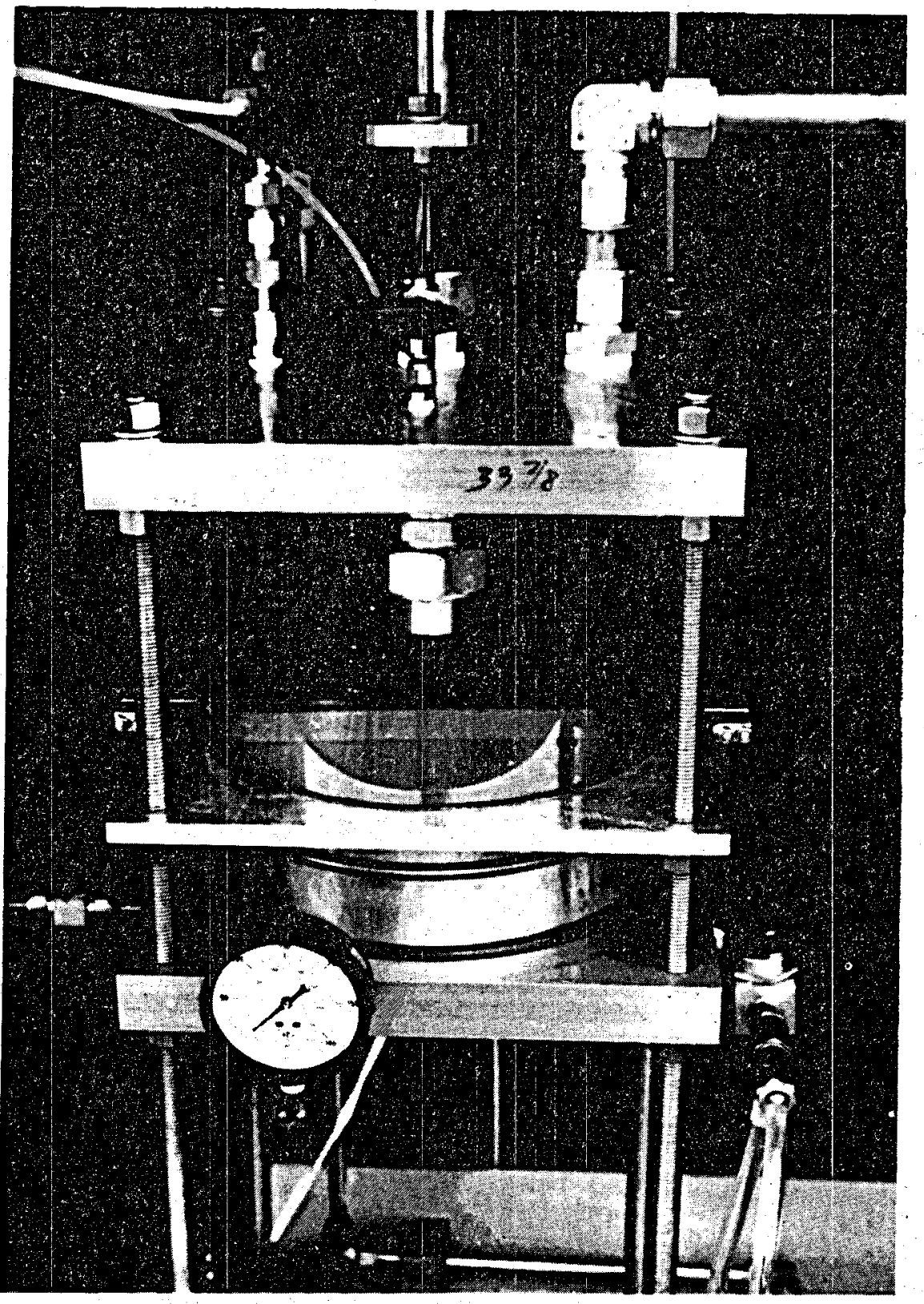

FIGURE 38 - CLEAR ACRYLIC TEST CHAMBER IN CHIP HOLD-DOWN FACILITY 


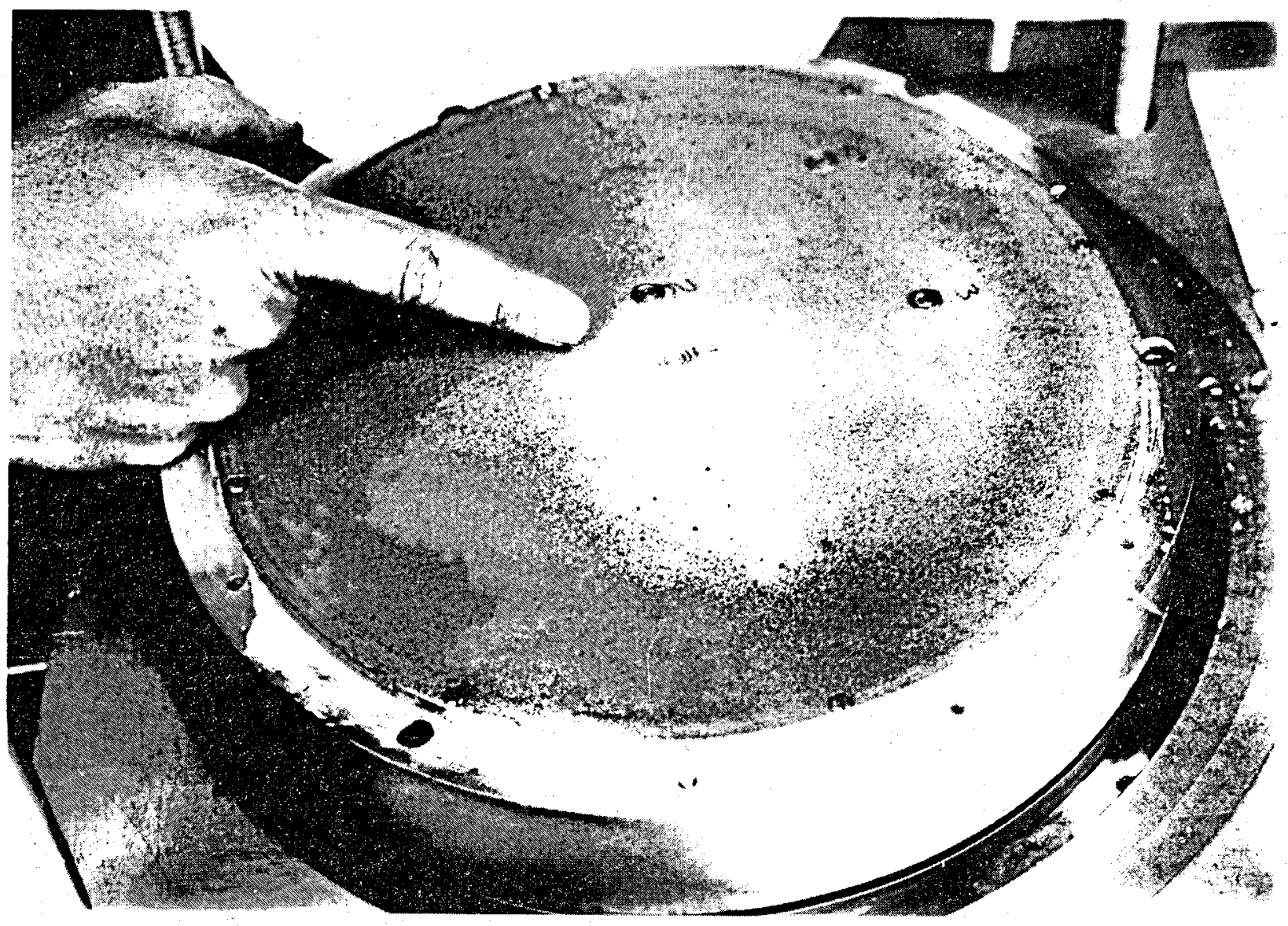

FIGURE 39 - PRESSURE TRANSDUCERS IN POROUS METAL SUPPORT PLATE OF CHIP HOLD-DOWN FACILITY 

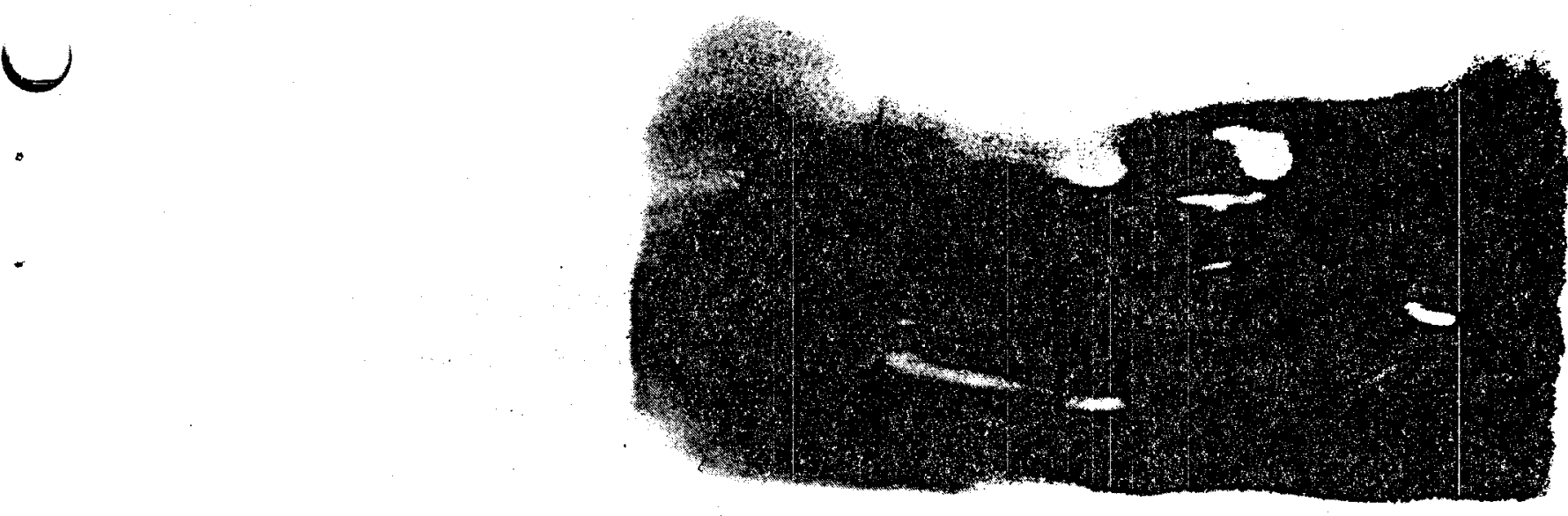

$$
\sigma=1.7
$$

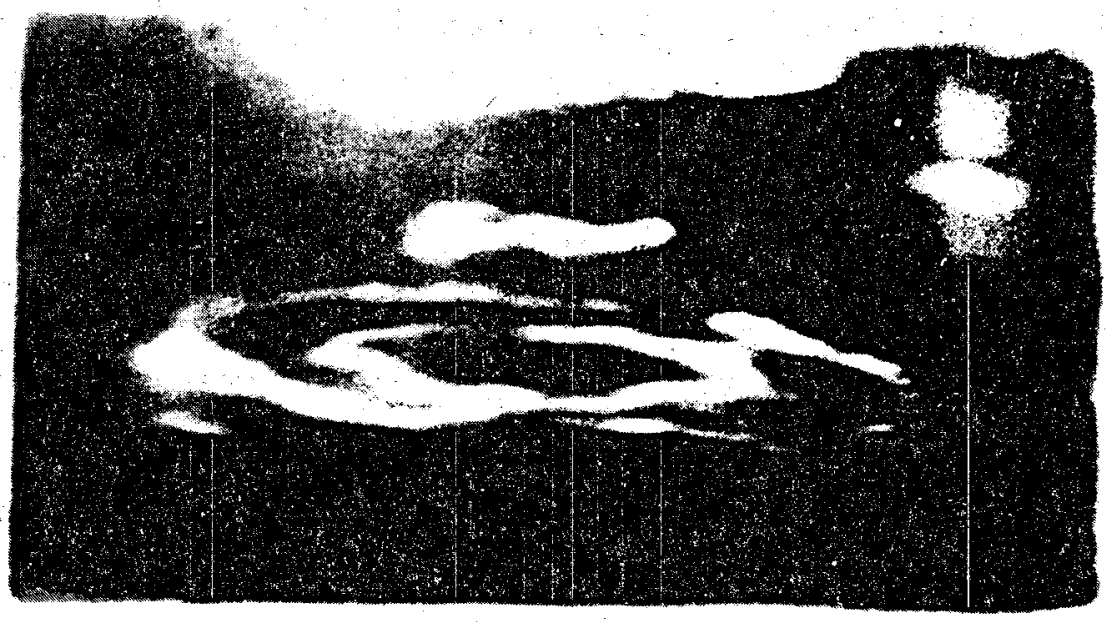

$$
\sigma=1.4
$$

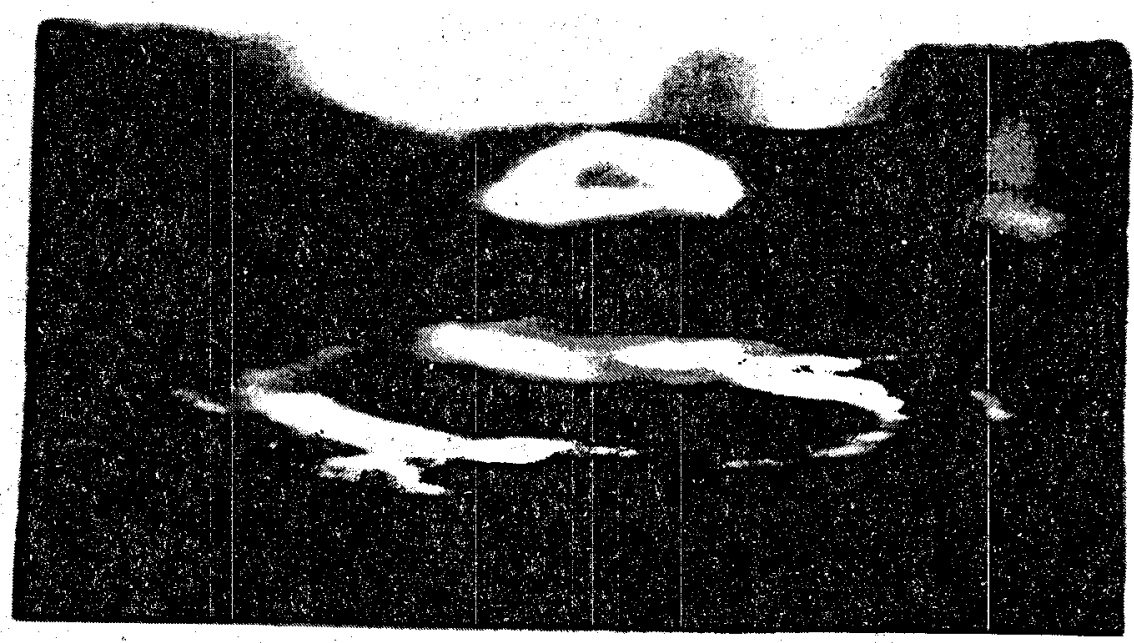

$$
\sigma=1.1
$$

FIGURE 40 - RING VORTICES SPREADING ON SURFACE AT $X / d=2.0, S_{d}=0.64$ 
STRATOJET or conventioral nozzles. The flow through the pump, up to $4.5 \mathrm{l} / \mathrm{s}(65 \mathrm{gpm})$, was regulated by a discharge valve to effect the desired nozzle pressure drop - up to $0.5 \mathrm{MPa}$ (75 psi) for the $12 \mathrm{~mm}$ ( $0.5 \mathrm{in.}$ ) diameter nozzles that were used.

Excellent visualization of the jet structure was afforded by this facility. Spreading of cavitating ring vortices on an impingement surface is illustrated in the photographs in Figure 40.

\subsection{Mud Bed Erosion}

Typical data from tests conducted in the chip hold-down facility are presented in Figures 41 and 42 . These data confirin that a structured STRATOJET can clean the hole bottom more effectively than an unstructured jet, even at very high cavitatior numbers (where there is no jet cavitation). In these tests, a thin (25 to 100 ) mud bed was formed over a woven nylon filter cloth that was supported by the porous stainless steel plate. The barite mud particle size distribution, measured with a Coulter Counter, ranged from 2 to $50 \mathrm{H}$ with maximum (volume) concentration around $8 \mu$. Nominal porosities (calculated pore area $\div$ total cell area, A) of the filter cloths were 0.13 and 0.01 respectively, for the $30 \mu$ and $5 \mu$ (maximum pore size) cloths.

The pores in the cloth were effectively clogged by the mud particles and caused a large pressure drop, $\Delta \mathrm{p}_{\mathrm{h}}$, to build up across the cloth. This "hold-down" pressure was regulated by the flow, $Q$ (up to $0.32 \mathrm{l} / \mathrm{s}(5 \mathrm{gPm})$ ), across the $235 \mathrm{~mm}$ ( $9 \frac{1}{4} \mathrm{in.}$ ) diameter mud bed. A failure of the mud bed was noted by a sudden change in bed porosity, $C_{p}=Q / A \sqrt{2 \Delta \mathrm{p}_{h} / \rho}$, induced by slowly increasing the jet driving pressure, $\Delta \mathrm{p}$. This porosity coefficient may be likened to the product of the effective pore area $\div$ surface area and a discharge coefficient. Between test runs the mud bed could reform (with the jet off), but the porosity also changed due to modifications in the particle distribution. 


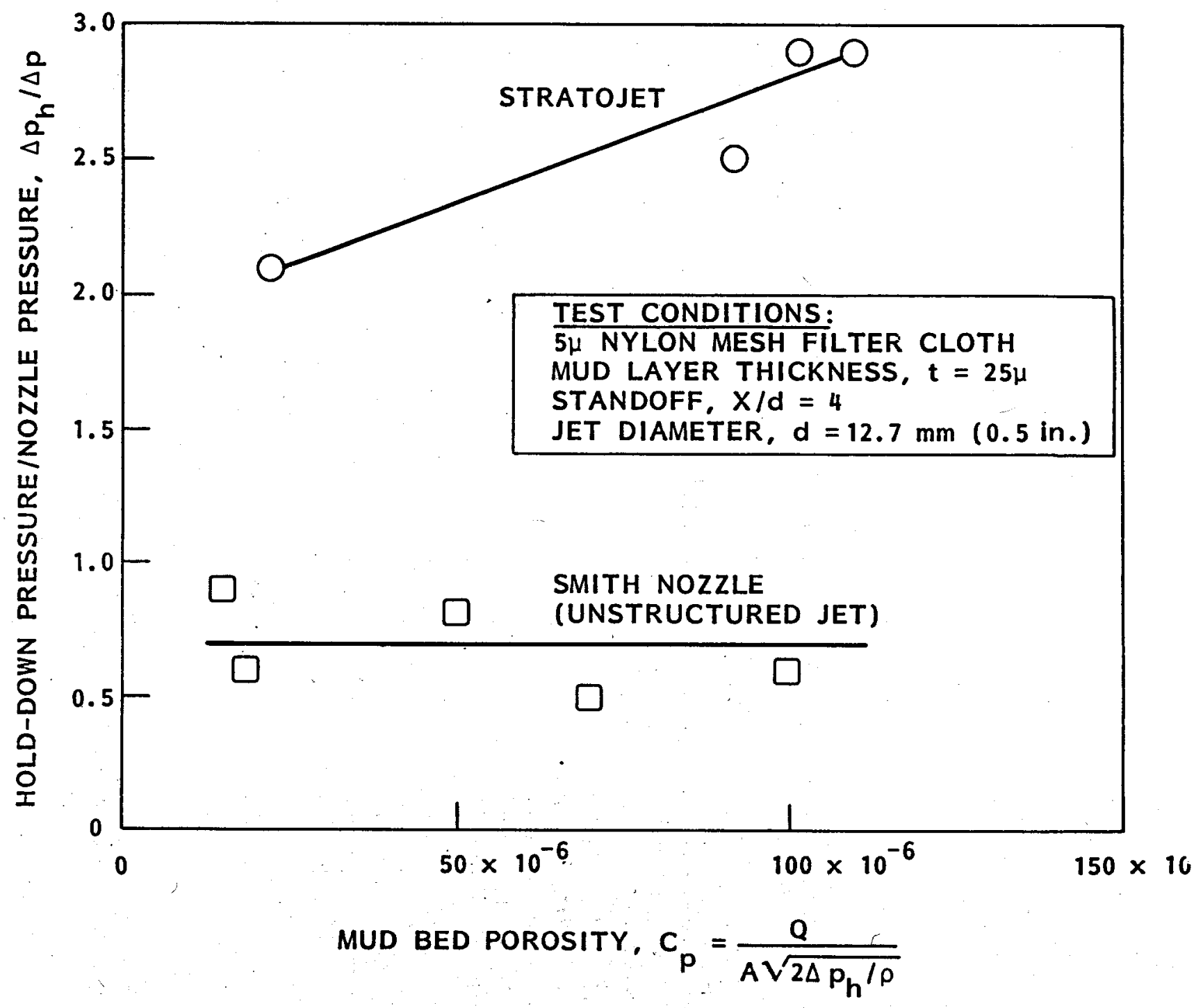

FIGURE 41 - COMPARISON OF MUD BED PENETRATION FOR UNSTRUCTURED AND STRUCTURED JETS, $5 \mu$ CLOTH 


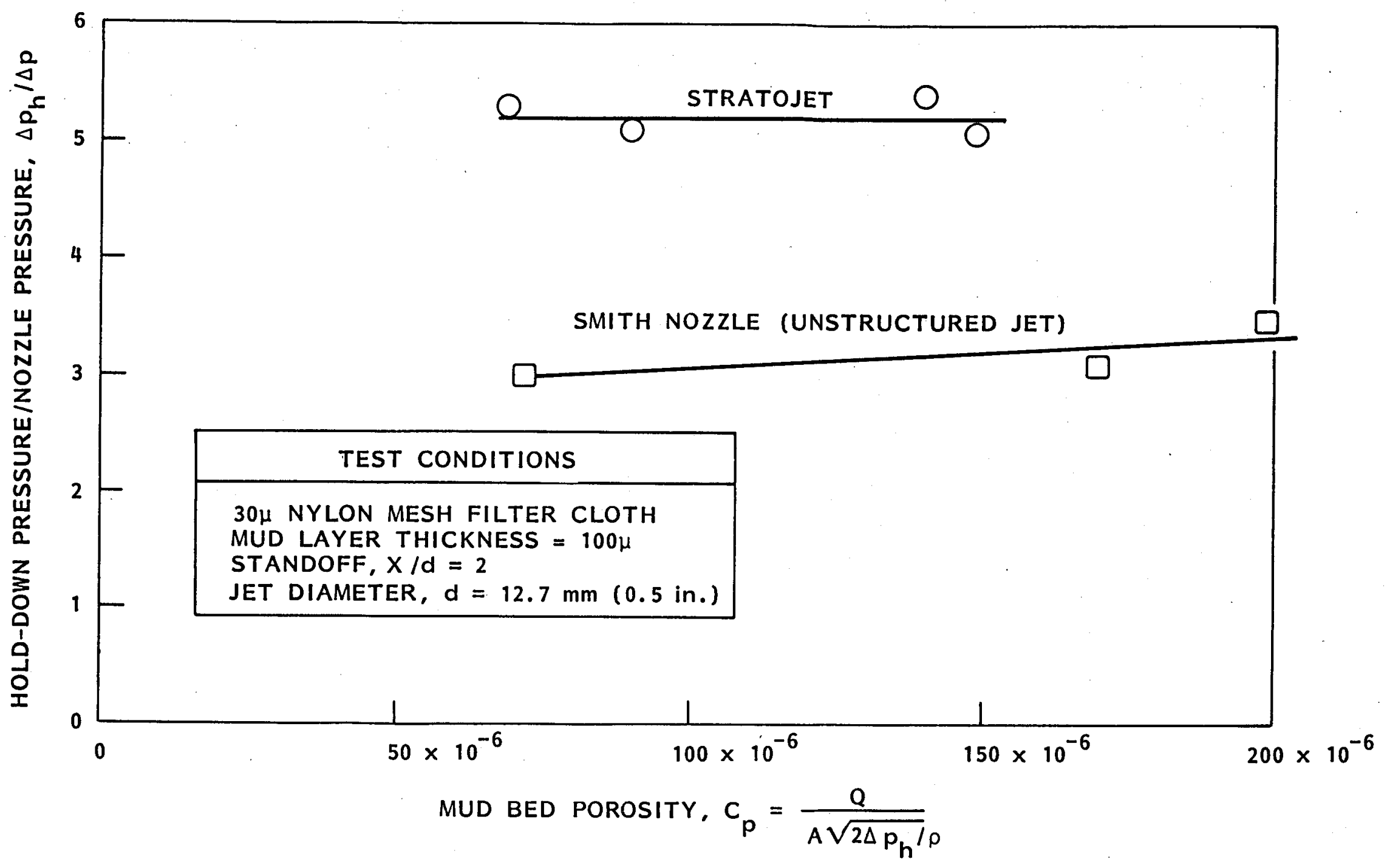

FIGURE 42 - COMPARISON OF MUD BED PENETRATION FOR UNSTRUCTURFN AND STRUCTURED JETS, 30 $\mathrm{CLOTH}$ 
Smaller particles could be lost through the pores of the mesh, particularly with the $30 \mu$ cloth.

The data in Figures 41 and 42 have been normalized to show the relative erosivity of the jet versus mud bed porosity, where the bed erosion strength was assumed to be proportional to the hold-down pressure. Hence, we have plotted the ratio of the mud bed pressure drop, $\Delta \mathrm{p}_{\mathrm{h}}$, to the jet driving pressure, $\Delta \mathrm{p}$, (at the point of bed failure) versus the bed porosity (before failing). For the purpose of estimating the degree of cavitation in these tests, $\sigma \simeq \Delta \mathrm{p}_{\mathrm{h}} / \Delta \mathrm{p}$ will be used. Cavitation inception was observed at $\sigma_{i}=3.5$ for the modulating STRATOJET and 0.5 for the unstructured SMITH nozzle. Thus, in Figure 41, the two nozzles were operating near to or below inception; in Figure 42 both nozzles were noncavitating.

The bed erosion strength seems to be fairly independent of porosity, but the STRATOJET was three to four times more effective than the unstructured $j$ et for the case shown in Figure 41. Here, the nominal rms modulation strength (measured with a pressure transducer mounted in the plate beneath the filter cloth) was observed at the jet centerline to be 16 percent of the mean impact pressure. For the test parameters in Figure 42, the erosivity of the STRATOJET was nearly twice that of the unstructured jet. The relative erosion strength of the mud bed formed on this $30 \mu$ mesh must have been less than with the $5 \mu$ mesh; changes to the critical hold-down/jet pressure ratio arising from variations in the standoff and mud layer thickness were found to be rather small. As a result, the mud bed failure was achieved with non-cavitating jets in Figure 42.

\subsection{Modulated Pressure Distributions}

Several large scale STRATOJET nozzles were tested in air; orifice diameters were 51 to $64 \mathrm{~mm}$ (2 to 2.5 in.). Pressure fluctuations measured just upstream of the nozzle contraction were observed to exceed the mean nozzle pressure drop by more than 200 percent, corresponding to rms velocity fluctuations in 
the jet as great as 50 percent of the mean speed (on the jet centerline, one to two diameters downstream of the orifice). The peak in normalized rms pressure fluctuation usually occurred at a Strouhal number 10 to 20 percent higher than for the maximum velocity fluctuation intensity.

In general, the nozzle configurations for optimal selfmodulation at the large scale were very nearly the same as those derived for full scale bit nozzles tested in air.

Data for the mean, $\bar{p}$, and fluctuating, $\mathrm{P}^{\prime}$, (rms) pressure distributions on a flat plate target are presented for two standoff distances in Figures 43 and 44. In each figure we have compared the performance of a steady jet and a strongly modulated jet $\left(u^{\prime} / V \simeq 0.50\right)$. In the first figure the impingement surface was just 1.2 orifice diameters from the nozzle exit. The pressure data have been normalized by the pressure drop across the nozzle, $\Delta p$, including the organ pipe feed tube length. The mean pressure fell rapidly from the peak centerline value to near zero at a radius about twice the nozzle radius for both the steady and structured jets. The fluctuating pressures were very dissimilar, however. For the steady case, the turbulent (mixing) zone had not yet diffused to the center of the jet, so $\mathrm{P}^{\prime}$ was minimal at $\mathrm{r}=0$ and strongest around $\mathrm{r}=1.5(\mathrm{~d} / 2)$. The fluctuations were random, broad band noise.

For the structured jet, the peak $\mathrm{p}^{\prime}$ was at the centerline where the signal was nearly a pure sine wave, hence, the normalized amplitude was $0.42 \sqrt{2}=0.59$ and the instantaneous pressure varied from $0.20 \Delta \mathrm{p}$ to $1.38 \Delta \mathrm{p}$. Of greater significance was the instantaneous pressure for the secondary peak in $\mathrm{p}^{\prime}$ at $\mathbf{r}=$ $1.5(\mathrm{~d} / 2)$ as shown in the inset of Figure 43 . The signal was not sinusoidal here, but exhibited large negative pressure spikes corresponding to the passage of the expanding vortex rings across the surface. The pressure ranged from $-0.9 \Delta p$ to 


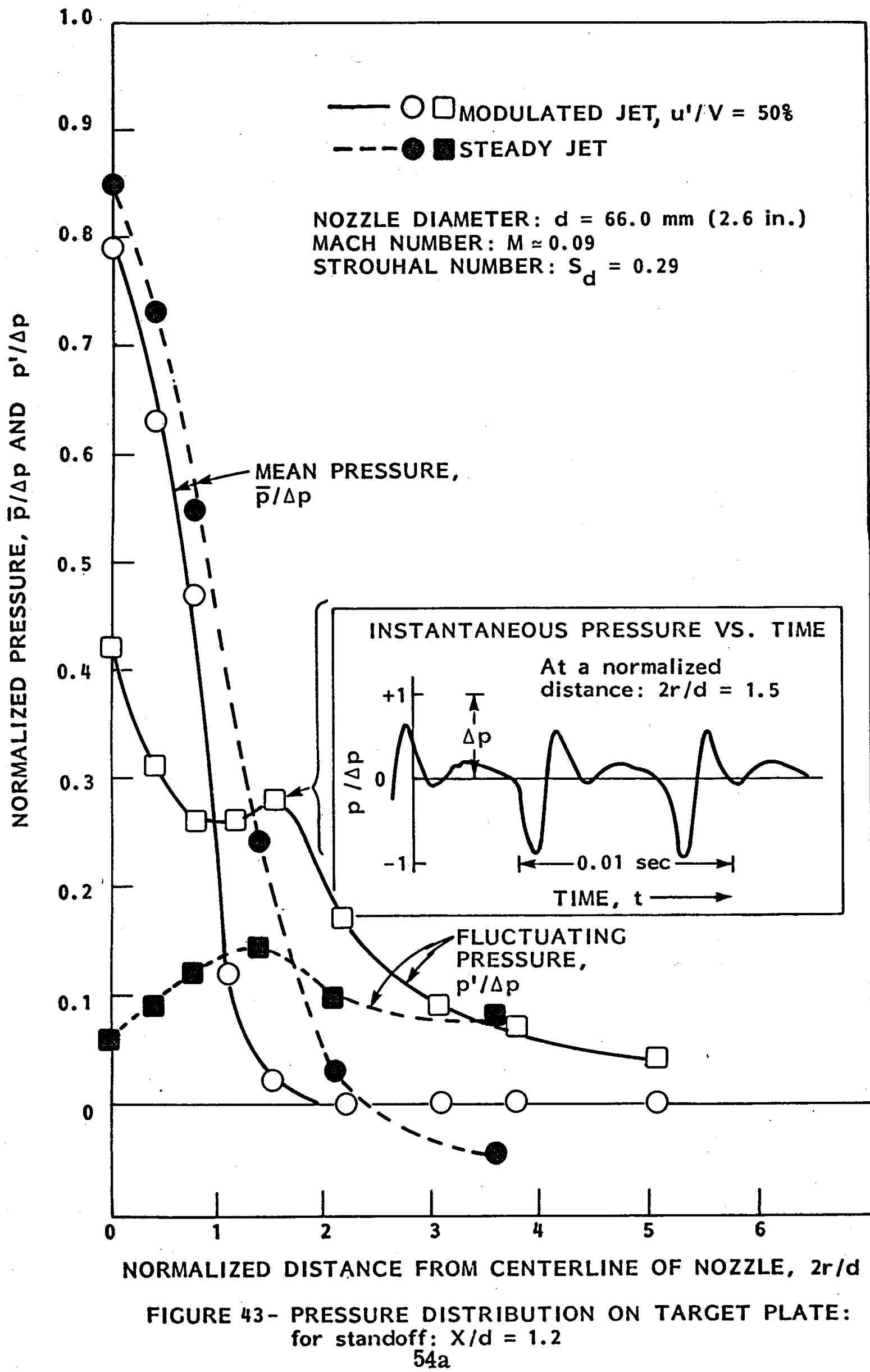




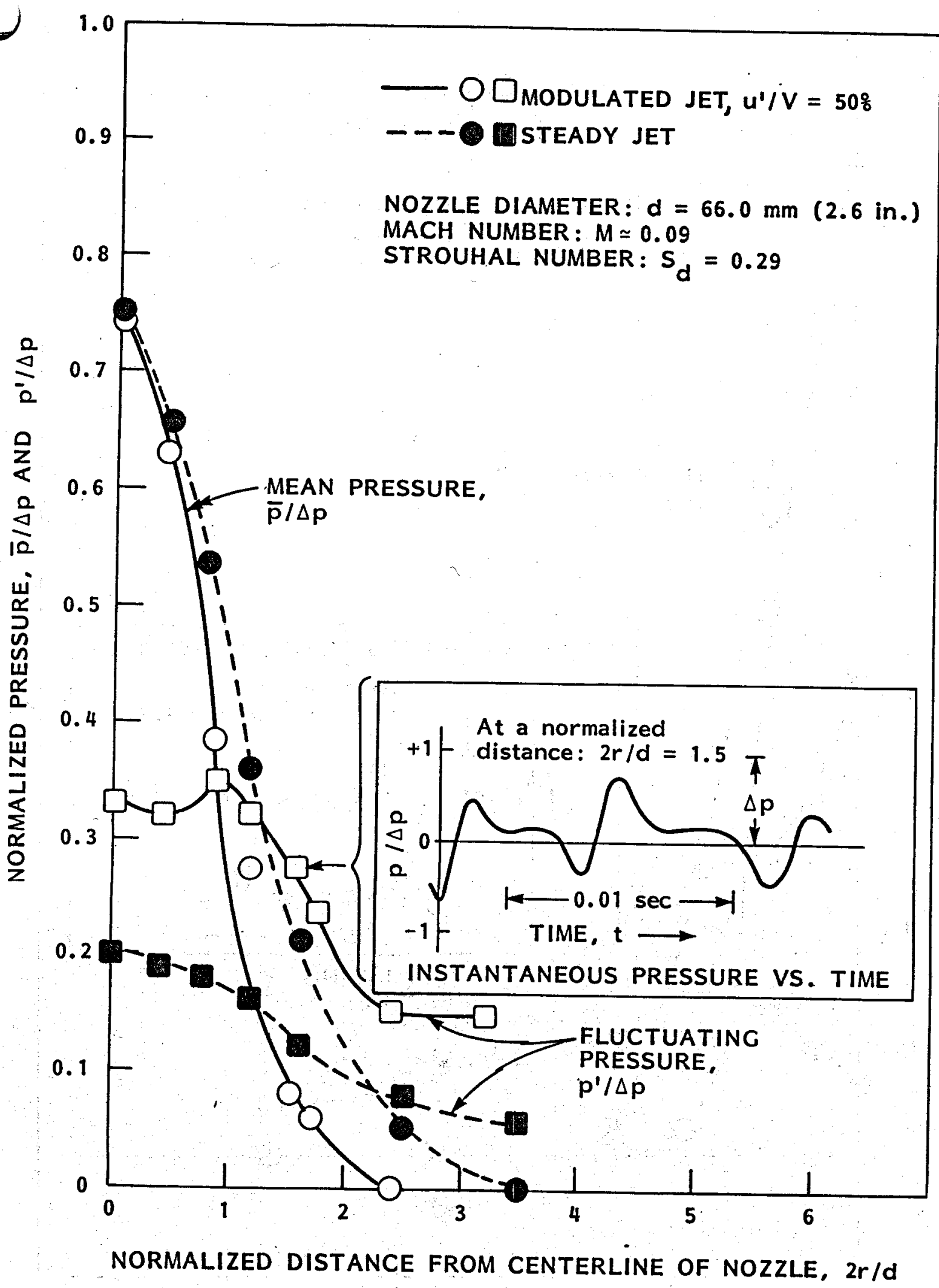

FIGURE 44- PRESSURE DISTRIBUTION ON TARGET PLATE: for standoff: $X / d=2.9$ 
$+0.5 \Delta \mathrm{p}$

With the target further downstream, $X=2.9 \mathrm{~d}$, the amplitude of the negative pressure peak was not as large. Here, as shown in the inset in Figure 44, the pressure ranged from around $-0.5 \Delta \mathrm{p}$ to $+0.5 \Delta \mathrm{p}$.

These data are significant from the standpoint of bottom hole cleaning - with or without cavitation in the vortex rings of the modulated jet. The large negative pressure peaks seen in the inserts in Figures 43 and 44 should contribute to lifting a chip from the hole bottom, by momentarily reducing the local hold-down pressure. 


\subsection{POLYCRYSTALLINE DIAMOND COMPACT (PDC) BIT TESTS}

Laboratory drilling tests with full-scale bits under simulated downhole conditions at Terra Tek's Drilling Research Laboratory (DRL) were undertaken to help assess the effects of replacing conventional nozzles in mechanical bits with improved CAVIJET nozzles. The selection of bits, rock type and test conditions were coordinated with Sandia and NL/HYCALOG personnel. The bit type chosen for these tests had polycrystalline diamond compact (PDC) cutting elements, specifically STRATAPAX cutters manufactured by the General Electric Company. The bits were experimental designs, provided by NL/HYCALOG, which had the brand name of JETPAX. Mancos shale was selected as the rock to be drilled because data for comparative purposes had been obtained at DRL on this same formation. The tests were run at DRL in December, 1980.

\subsection{Bit Test Parameters}

Three $21.6 \mathrm{~cm}\left(8 \frac{1}{2}-i n.\right)$ diameter PDC bits were provided for these tests. The bits were essentially identical except for the set of three carbide nozzle inserts that were cast into the matrix of each tool. The three nozzle types, depicted (in cross-section) in Figure 45 were: a) PULSER CAVIJETS, b) Plain CAVIJETS, and c) Standard drill bit nozzles (manufactured by the Hughes Tool Company). The two CAVIJET nozzle types had diameters of $11.9 \mathrm{~mm}(0.47 \mathrm{in.})$ to give a discharge equivalent to the standard $9.5 \mathrm{~mm}$ (12/32-in.) nozzles. Thus, the effects of nozzle type could be assessed directly when tests were run under identical conditions and flow rates.

The three-bladed PDC bits were an experimental design with a relatively small number (19) of the $19 \mathrm{~mm}$ (3/4-in.) diameter STRATAPAX cutters. Two of these bits are shown in Figure 46 . 


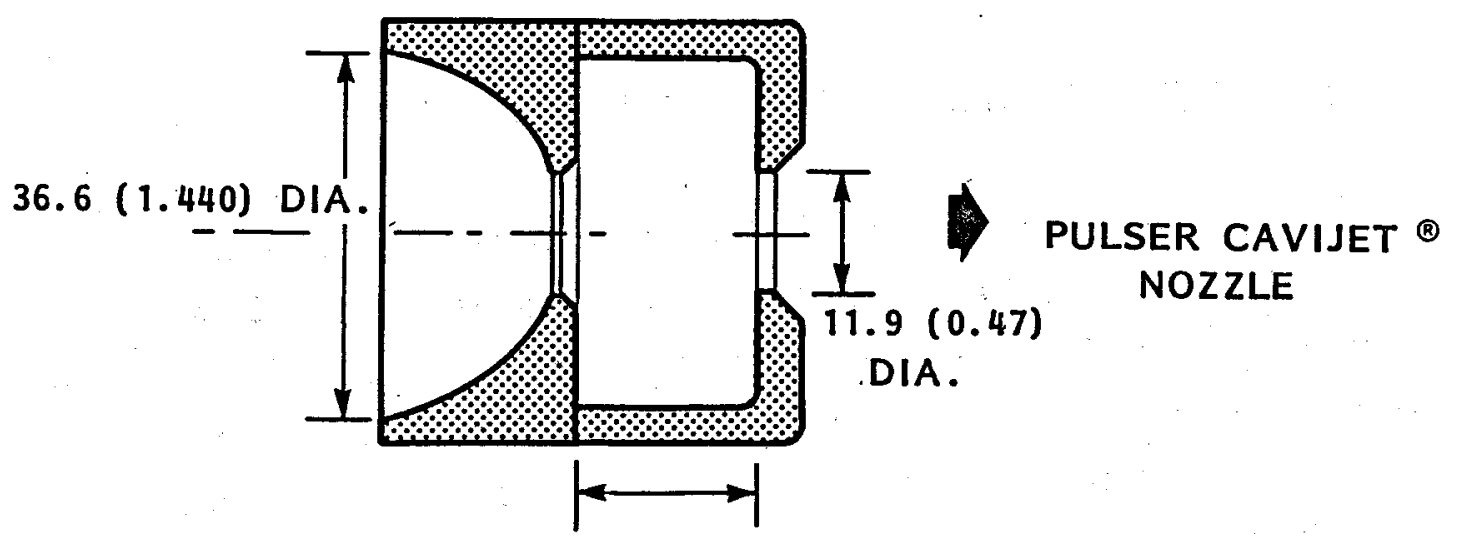

ALL DIMENSIONS ARE mm (in.)
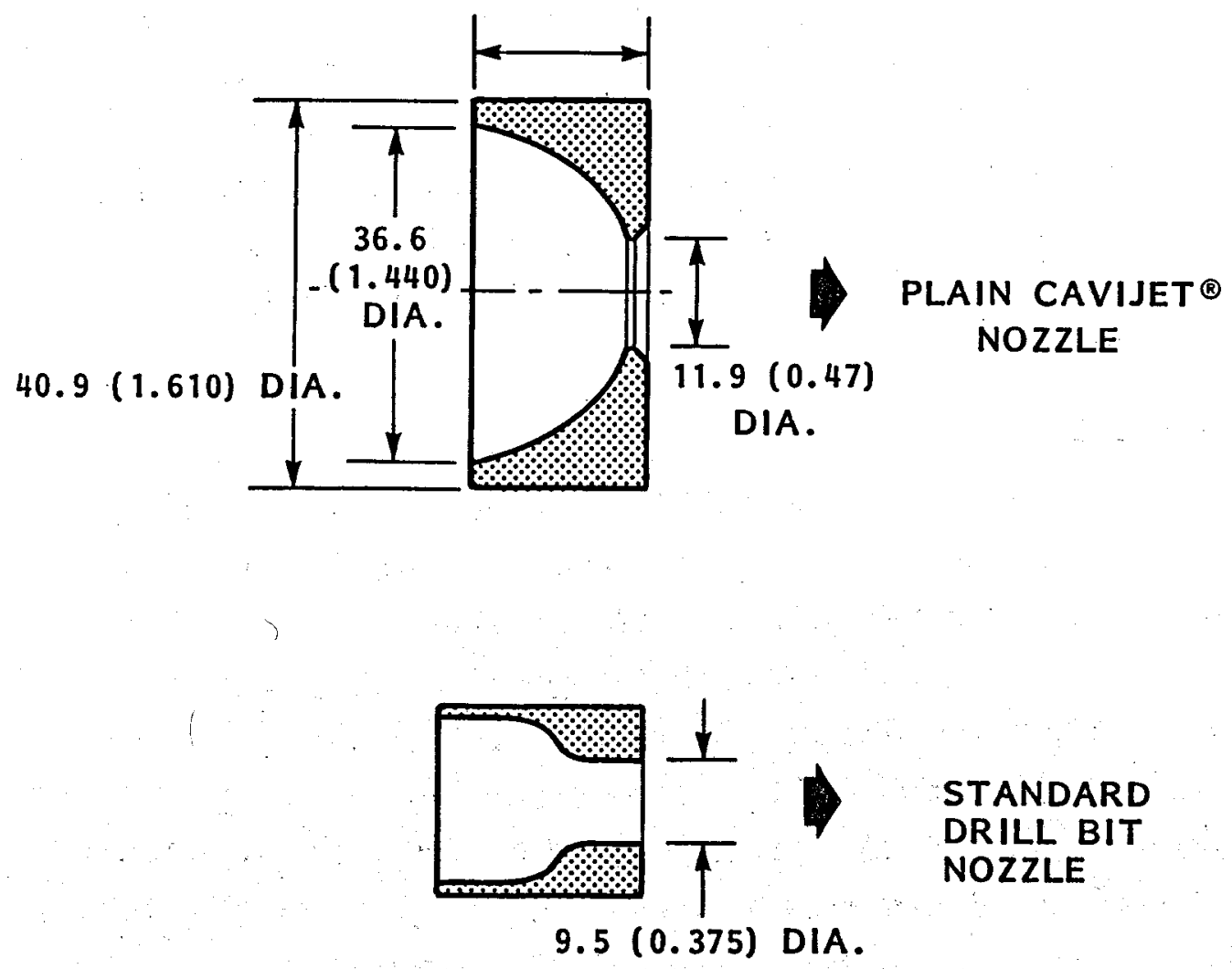

FIGURE 45 - NOZZLES FOR EXPERIMENTAL $21.6 \mathrm{~cm}$ ( $8 \frac{1}{2}$ in.) PDC BITS 


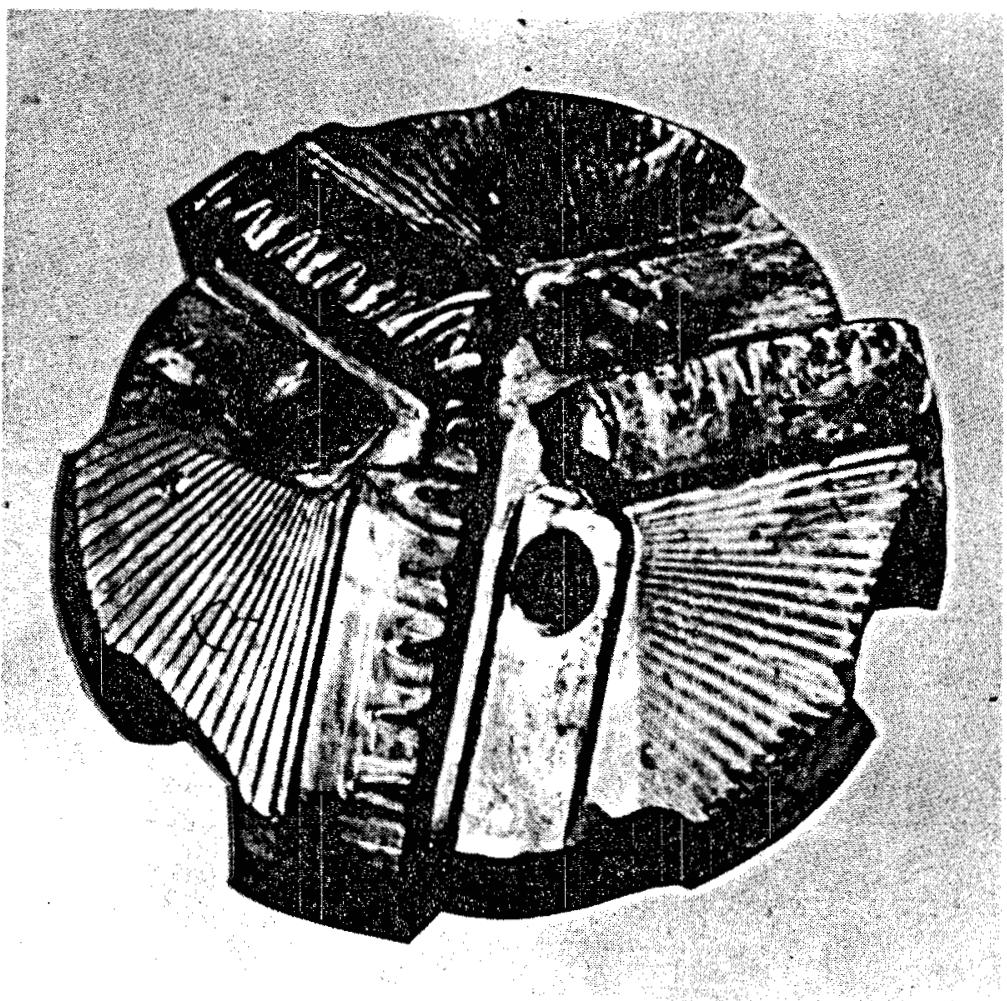

a. PULSER CAVIJET NOZZLES

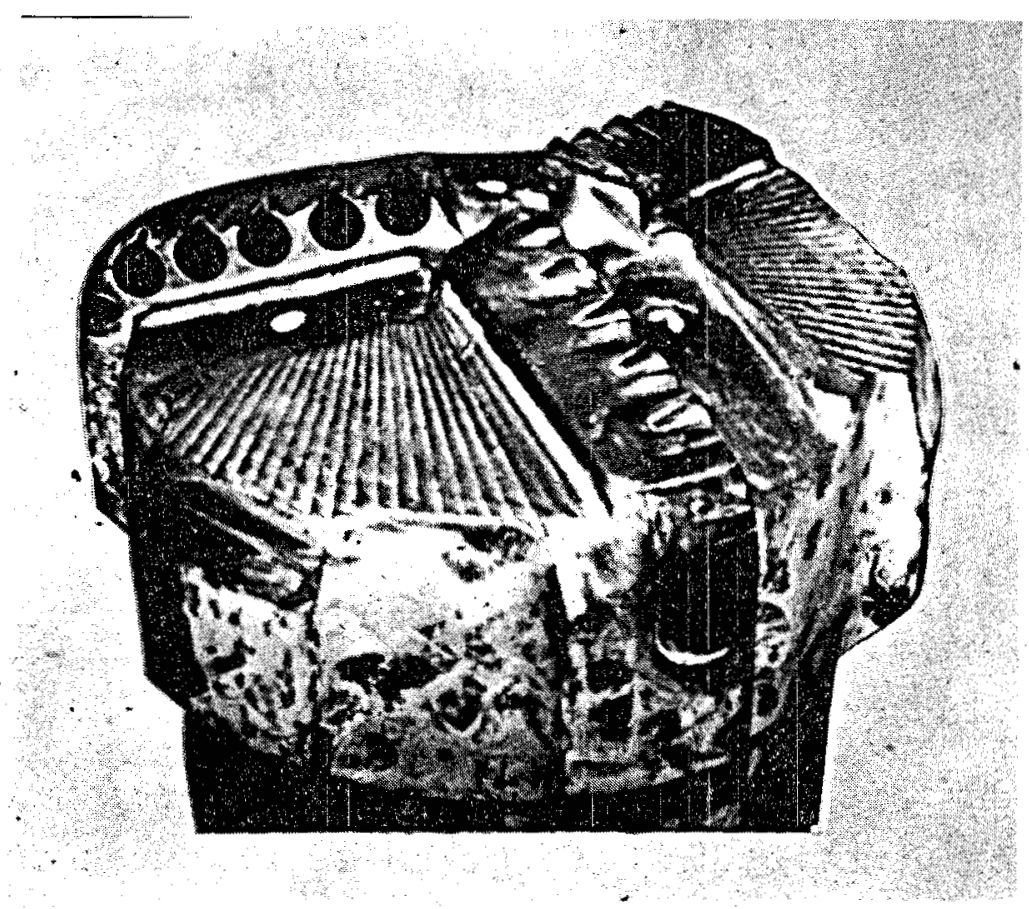

b. STANDARD NOZZLES

FIGURE 46 - EXPERIMENTAL $21.6 \mathrm{~cm}$ ( $8 \frac{1}{2}$ in.) PDC BITS 
Tests were run in six Mancos shale specimens - two specimens for each of the three PDC bits. From 14 to 21 individual runs were obtained in each specimen; a run lasted several seconds during which time about 2 to $5 \mathrm{~cm}$ ( 1 to 2 in.) of shale were drilled. The variable test parameters for the various runs were repeated (nominally) for each of the three bits. They were:

\section{PARAMETER}

Bore Hole Pressure $\left(p_{a}\right)$

Ram Pressure

Confining Pressure

Nozzle Pressure Drop $(\Delta \mathrm{p})$

Mud Flow

Hydraulic power per bit area

Weight on Bit (WOB)

Rotative Speed
RANGE

2.5 to $28.5 \mathrm{MPa}$

(365 to 4,140 psi)

$2.0 \mathrm{pa}_{\mathrm{a}}$

$1.4 \mathrm{pa}_{\mathrm{a}}$

2.1 to $12.7 \mathrm{MPa}$

(300 to $1,840 \mathrm{psi}$ )

17.7 to $33.1 \mathrm{l} / \mathrm{s}$

(280 to $525 \mathrm{gpm}$ )

0.8 to 9.4 HSI*

20.5 to $48.9 \mathrm{kN}$

$(4,600$ to $11,0001 \mathrm{~b})$

47 to 143 RPM

The drilling fluid was $1.13 \mathrm{gm} / \mathrm{cm}^{3}(9.4 \mathrm{ppg})$ water-based mud with 15 to 18 centipoise apparent viscosity.

The dependent variable was the drill bit performance in terms of rate of penetration, ROP, usually measured in

* Since HSI (hydraulic horsepower per in $^{2}$ ) is the conventional unit for this parameter, a metric conversion will not be used. The "bit area" is the projected surface area of the bit on a plane perpendicular to the drill-pipe axis, 
meters/hour, (feet/hour). A related measure is the tool's depth of penetration per shaft revolution ("DOP") reported as either $\mathrm{mm}$ per revolution (mm/rev.) or inches per revolution (in./rev.). The DOP is closely tied to the actual depth of penetration of the PDC cutters into the formation: We found that the PDC bit penetration test results were correlated strongly with the curmulative depth of penetration of each bit into the Mancos shale specimens. It was found that the bits were tested over a period when "wear-in" was a dominant factor in the effective exposure of the cutters. Excess matrix material on the bit face, behind and alongside the cutters, was continuously being worn off or "rung out" of the tool. Until this material was removed, it supported some of the bit weight load and, thus, reduced the normal loading and penetration of the cutters.

\subsection{Test Results}

The independent test parameters were; (i) bit weight (WOB), (ii) rotative speed, (iii) bore hole pressure, (iv) hydraulic power per bit area (HSI), and (v) nozzle type. The first three of these affected the ROP or DOP in ways that may be discerned in the test data. The effect of hydraulic power, if any, was not clearly indicated and any nozzle type effect was uncertain because of possible differences in wear-in between the three bits tests.

6.2.1 Wear-in - The wear-in of each test bit is exhibited in Figures 47, 48, and 49, wherein the DOP is plotted as a function of the cumulative penetration in the shale specimens. The result for every test run is shown. To isolate the effect of wear-in from other test variables, a solid line has been drawn through the data for: 


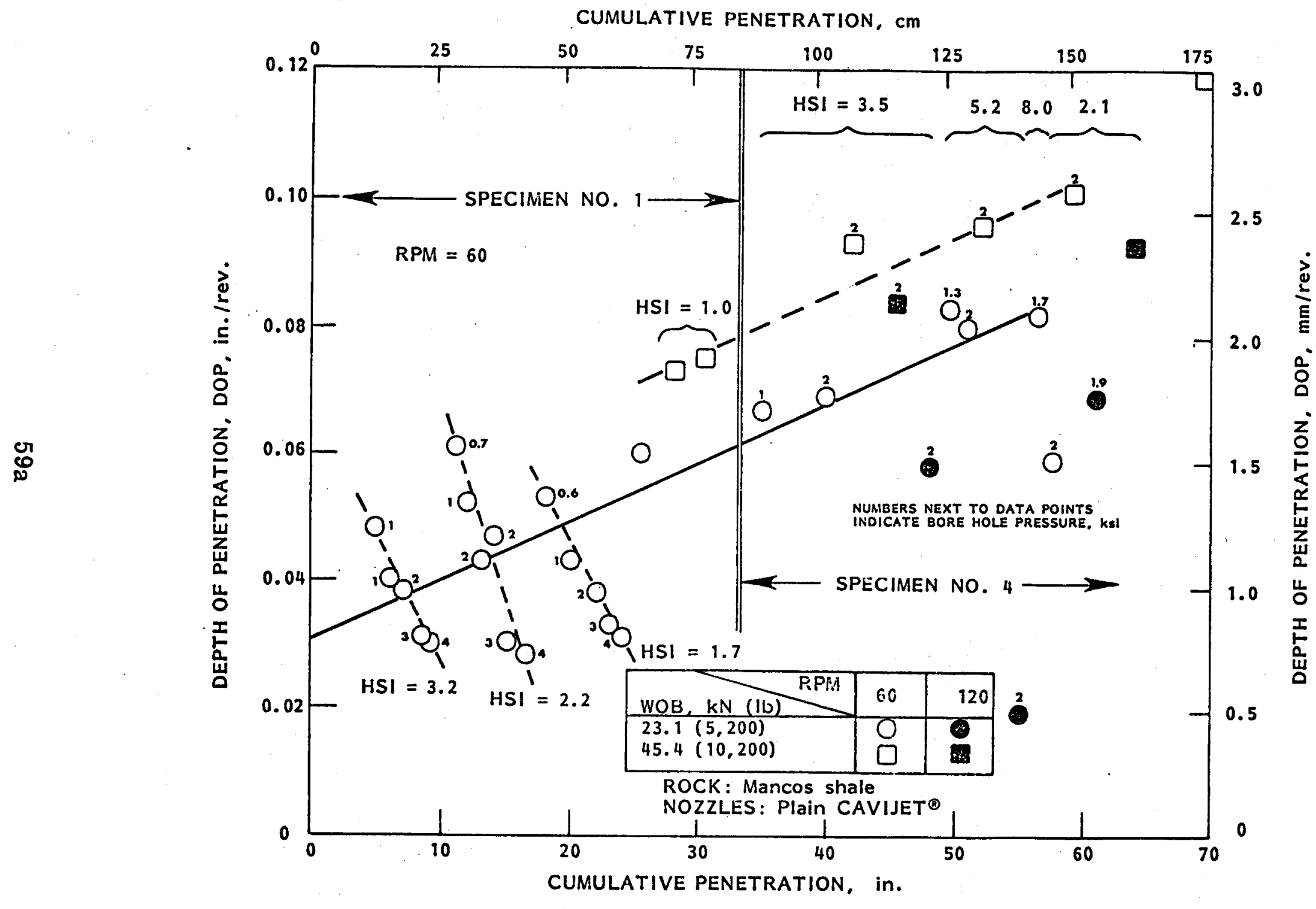

FIGURE 47 - WEAR-IN OF PDC BIT DURINC DRL TESTS; plain CAVIJET ${ }^{\circ}$ nozzles 


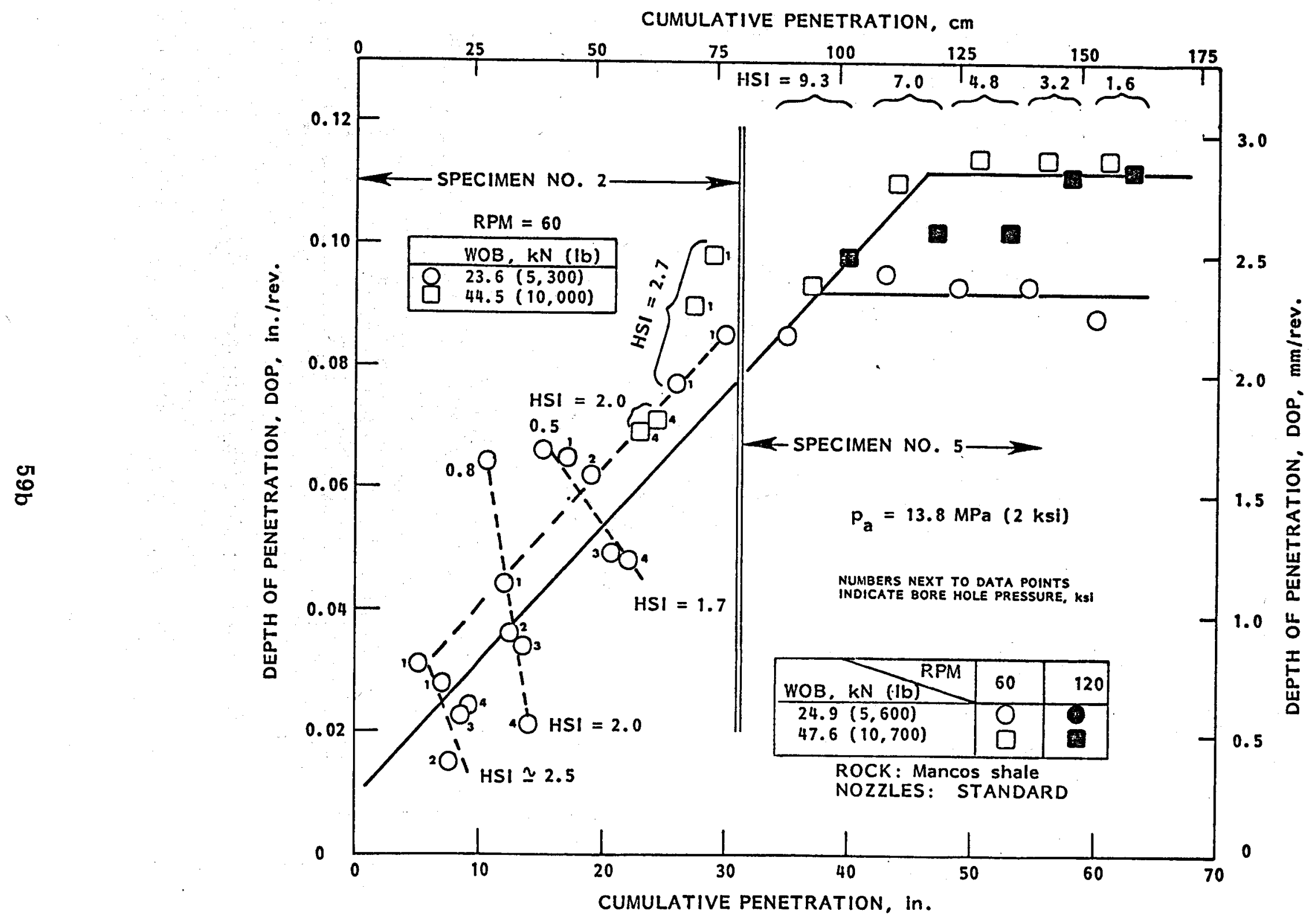

FIGURE 48 - WEAR-IN OF PDC BIT DURING DRL TESTS; stanciard drill bit nozzle 


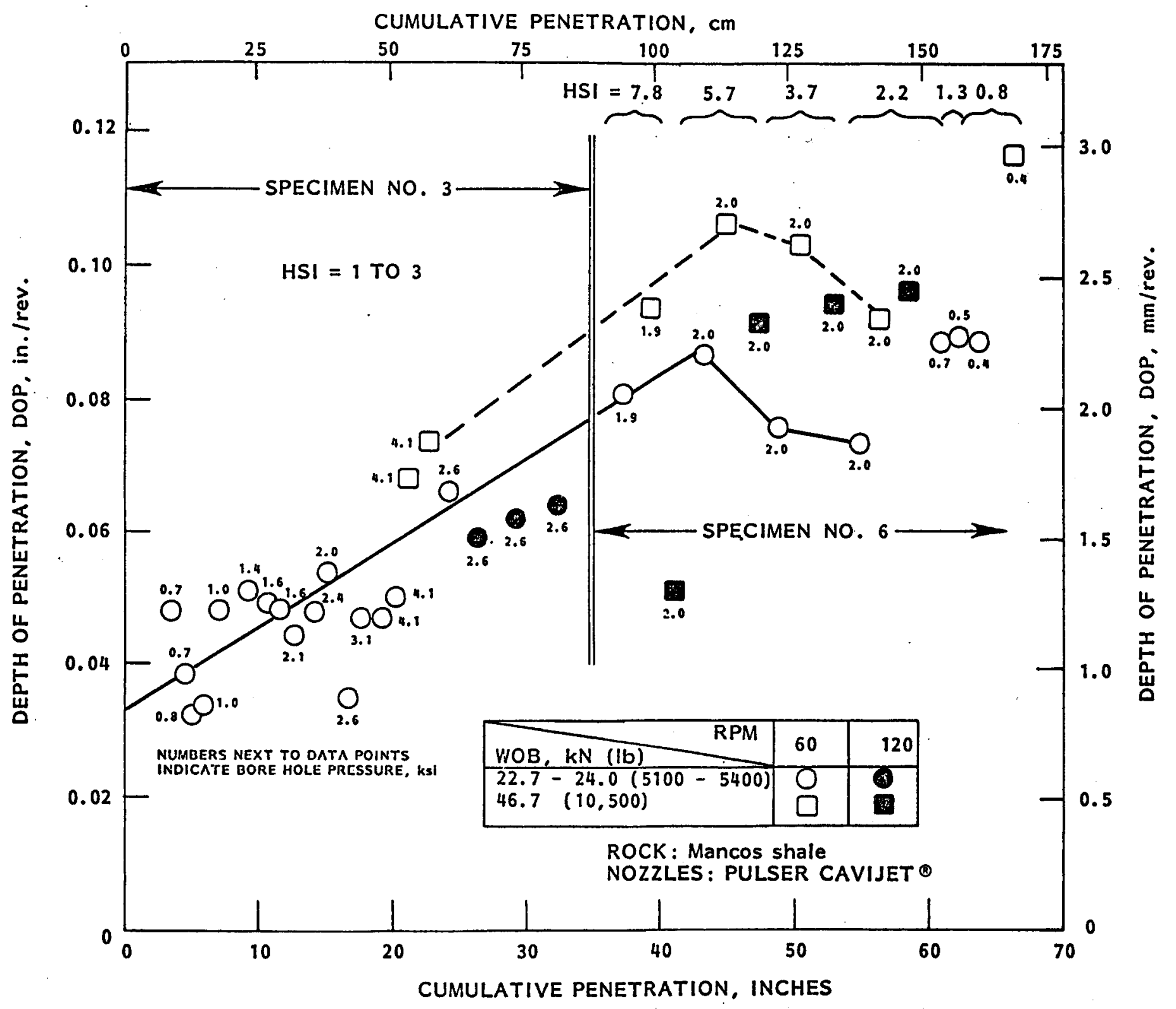

FICURE 49 - WEAR-IN OF PDC BIT DURING DRL TESTS;
PULSER CAVIJET ${ }^{\oplus}$ NOZZLES 
Bore hole pressure $=13.8 \mathrm{MPa}(2 \mathrm{ksi})$

Bit weight $\simeq 22.2 \mathrm{kN}(5000 \mathrm{lbs})$

$\mathrm{RPM}=60$

These conditions and data are taken as the standard or baseline for comparative purposes in the discussions that follow.

For each bit, the baseline DOP is seen to increase continuously in the first specimen with about $75 \mathrm{~cm}$ (30 in.) of penetration. DOP continued to increase into the second specimen, but after about $100 \mathrm{~cm}$ (40 in.) of penetration the DOP leveled off (Figure 48) or even declined (Figure 49) (the decline may have been caused by reductions in hydrualic horsepower per square inch, HSI).

The rate at which DOP increased was greatest for the bit with standard nozzles (Figure 48), going from about 0.5 to 2 $\mathrm{mm} / \mathrm{rev}$. (0.02 to $0.08 \mathrm{in./ \textrm {rev } . )}$ in Specimen No. 2. Wear-in of the bit with plain CAVIJET nozzles (Figure 47) was more gradual and $2 \mathrm{~mm} / \mathrm{rev}$. (0.08 in./rev.) was achieved only after about $127 \mathrm{~cm}$ (50 in.) of total penetration.

6.2.2 Bore hole pressure - The ambient bore hole pressure was systematically varied during the drilling tests on Specimen Nos. 1 and 2 (Figures 47 and 48 ). The DOP is seen to decrease with increasing pressure as indicated by the dashed lines through sets of data points at constant values of HSI. These data, normalized and corrected for wear-in, are replotted as a function of bore hole pressure in Figure 50. The wear-in correction was made by dividing the measured DOP by the baseline DOP (for $\mathrm{P}_{\mathrm{a}}=13.8 \mathrm{MPa}(2 \mathrm{ksi})$ ) at the same cumulative penetration (from Figures 47 and 48); the resulting borehole pressure factor has a value of one at $\mathrm{P}_{\mathrm{a}}=13.8 \mathrm{MPa}(2 \mathrm{ksi})$ by definition. The results suggest that DOP is roughly proportional 


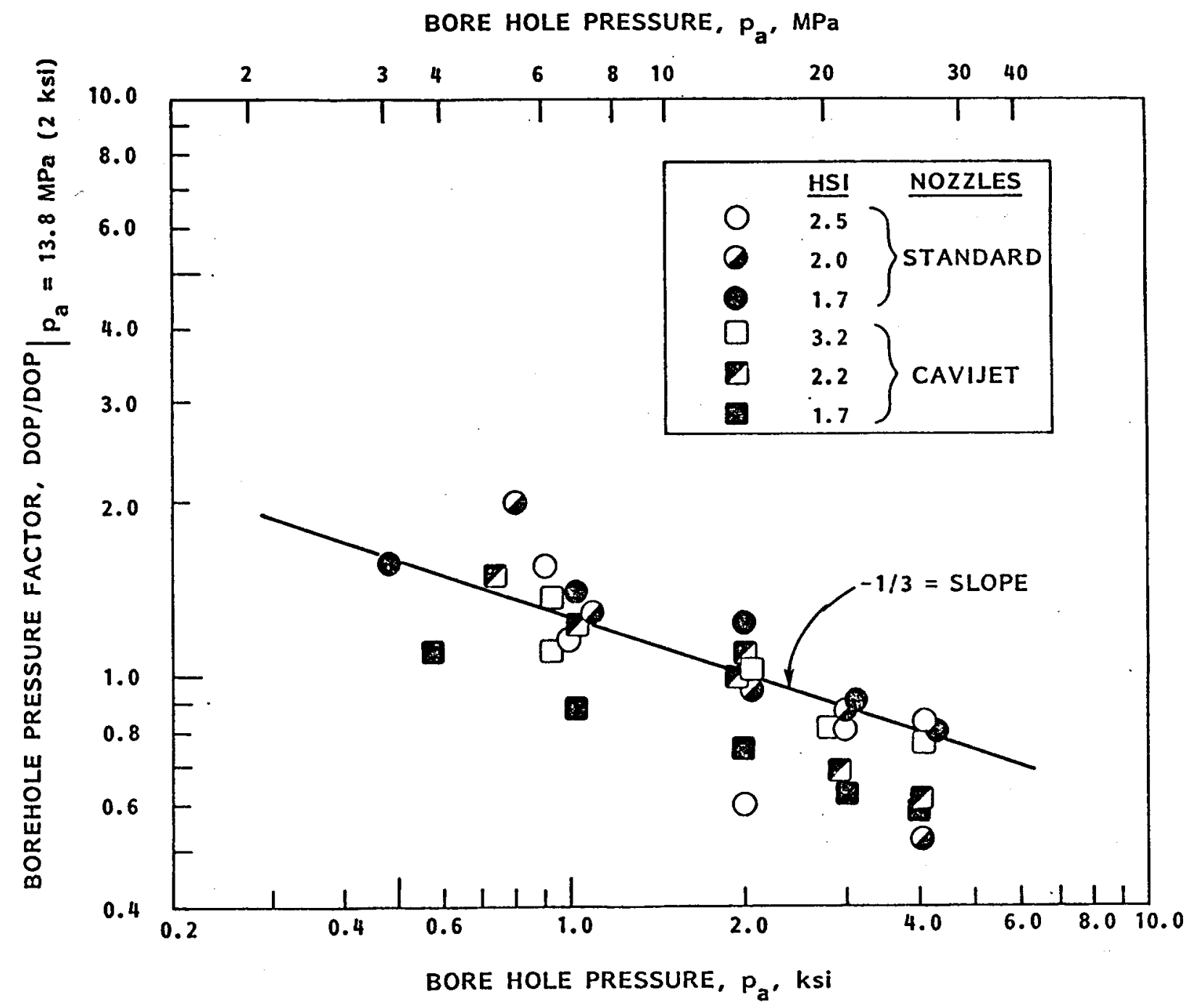

FIGURE 50 - EFFECT OF BORE HOLE PRESSURE ON PDC BIT PENETRATION 
to $\left(p_{a}\right)^{-1 / 3}$ in the range that was tested.

Similar tests in the Mancos shale performed at DRL with three-cone roller bits showed an even stronger dependence, with ROP proportional to $\left(\mathrm{p}_{\mathrm{a}}\right)^{-2 / 3}$ in the range 3.4 to $27.6 \mathrm{MPa}$ (0.5 to. $4 \mathrm{ksi}$ ) (see Reference 18 ).

6.2.3 RPM - The DOP is seen to decrease by zero to twenty percent when the bit speed is increased from 60 to 120 RPM (compare open symbols to filled symbols in Figures 47, 48, and 49). Hence, the ROP is proportional to $(\mathrm{RPM})^{s}$, where $s=0.7$ to 1.0 in the range that was tested.

6.2.4 Bit weight - Doubling the bit weight from about 22.2 to $44.5 \mathrm{kN}(5,000$ to $10,000 \mathrm{lb})$ caused the DOP to increase from 20 to 40 percent (compare circle and square symbols in Figures 47, 48, and 49). Thus, in the range tested, the ROP is roughly proportional to (WOB) $\left(\frac{3}{4}\right.$ to $\left.\frac{1}{2}\right)$.

6.2.5 Hydraulic power - There were some indications that decreasing HSI caused a decrease in DOP. For example, see the open symbols for $\mathrm{p}_{a}=13.8 \mathrm{MPa}(2 \mathrm{ksi})$ in Specimen Nos. 5 and 6 (Figures 48 and 49). The effect appears small, but may have been masked by continued bit wear.

\subsection{Summary of Results}

The rates of penetration (ROP) for these experimental PDC bits in Mancos shale were much faster than observed by DRL staff in comparable testing which they performed with threecone roller bits (Reference 18) and with a five-bladed STRATA$\mathrm{PAX}$ bit as illustrated in Figure 51 .

The three-bladed, experimental $21,6 \mathrm{~cm}\left(8 \frac{1}{2}-i \mathrm{n}_{1}\right)$ diameter JETPAX bit design used in the present study was relatively insensitive to hydraulic horsepower and hence pressure drop across the nozzles. This was not anticipated, and according to the 
WEIGHT ON BIT, $k N$

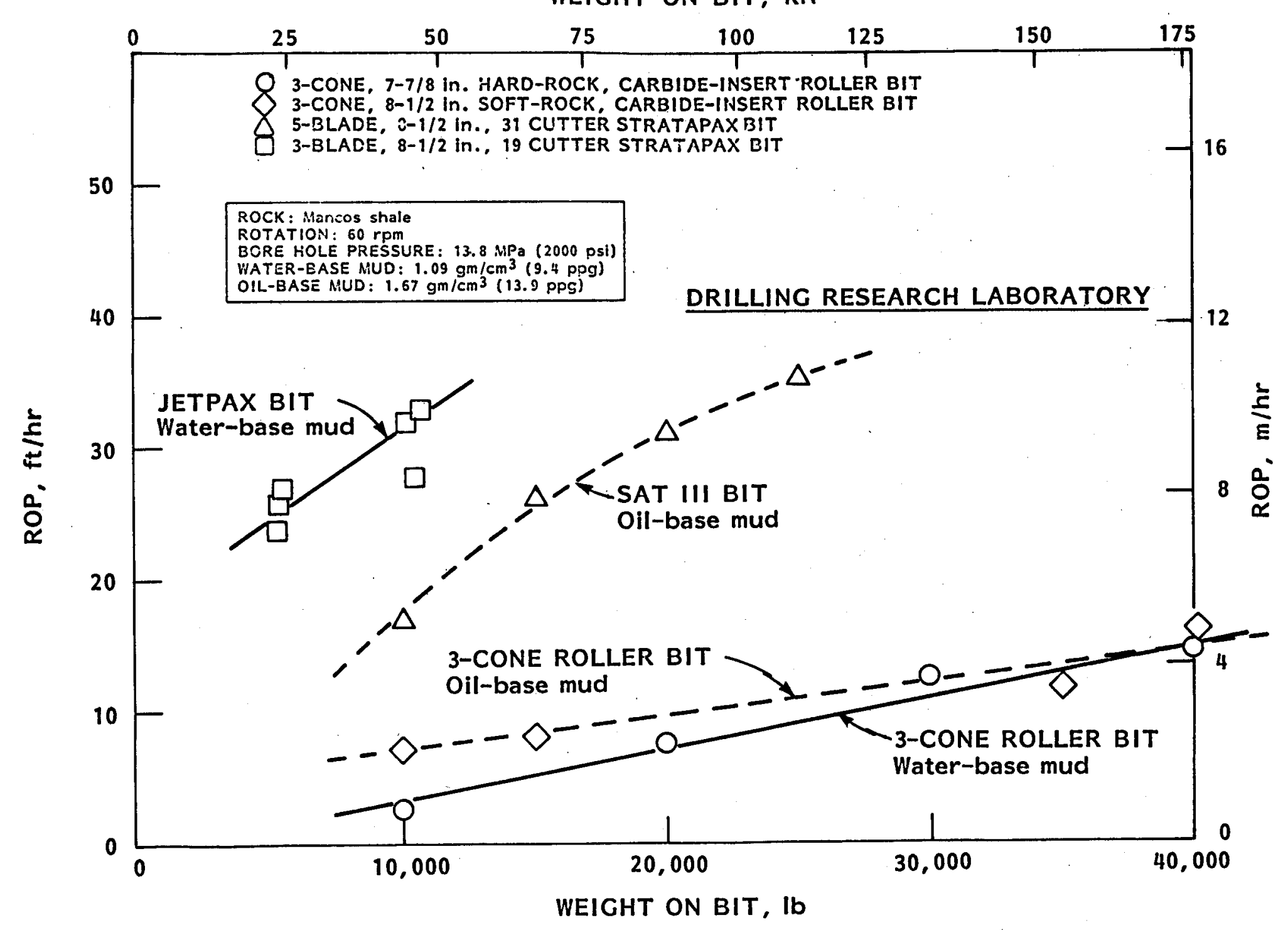

FIGURE 51 - COMPARISON OF DRILL BIT PERFORMANCE IN MANCOS SHALE 
$\mathrm{NL} / \mathrm{HYCALOG}$ engineer who participated in these tests, Mr. John Sherrill, it was a performance response which he had never seen before for such bits.

Bit wear-in, i.e., slight erosion of excess tool matrix material to allow full design penetration of the PDC cutters, was a strong factor in these tests. Indeed, linear correlations between cutter penetration and total distance drilled by each bit showed that, until completed, the wear-in effect masked all of the other operating parameters.

These factors, wear-in and relative insensitivity to hydraulics, therefore caused the tests to be inconclusive with regard to any enhanced bottom hole cleaning effects which mey be afforded by improved nozzle designs. 


\subsection{THREE-CONE BIT TESTS}

Continued developments in organ pipe self-resonance principles and nozzle design motivated a second series of drilling tests in the Vell Bore Simulator at DRL in December 1981 and January 1982. The capabilities at DRL had been upgraded by this time to allow

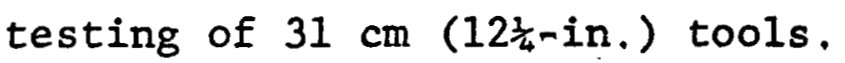

\subsection{Bit Test Parameters}

Two $31 \mathrm{~cm}$ ( $12 \frac{1}{2}$ in.) $\mathrm{F} 2 \mathrm{CE}$ three-cone roller bits were furnished by SMITH TOOL who shared the costs for these tests. The standard or baseline bit had three $8.7 \mathrm{~mm}(11 / 32-i n$.$) SMITH$ nozzles in the extended tubes and one $8.7 \mathrm{~mm}(11 / 32-$ in.) $(10.3$ $\mathrm{mm},(13 / 32$-in.) equivalent) center venturi nozzle (for cone cleaning). The modified bit had an identical center venturi nozzle. The only alterations to the baseline design were in the internal passages of the extended tubes leading to the three $9.5 \mathrm{~mm}$ (12/32-in.) diameter ORGAN-PIPE CAVIJET nozzles, as shown in Figure 52. The modified tube had a constant 20.6 $\mathrm{mm}$ (13/16-in.) internal diameter leading from the bit plenum to the nozzle, unlike the standard tube which necks down from 27.0 to 20.6 to $15.9 \mathrm{~mm}(17 / 16$ to $13 / 16$ to $5 / 8$-in.) between plenum and nozzle. The CAVIJET nozzles were sized to attempt to give the same discharge - when the flow is strongly modulated by organ pipe resonance - as standard jets at the same mean pressure drop across the nozzles. However, as discussed in Section 7.3 , the pressure fluctuations in these tests were smaller than anticipated, thus causing the total hydraulic power for the modified, ORGAN-PIPE CAVIJET bit to be less than that of the standard bit.

The bit modifications were made in accordance with guidance obtained from laboratory tests in air (full scale) and in water 

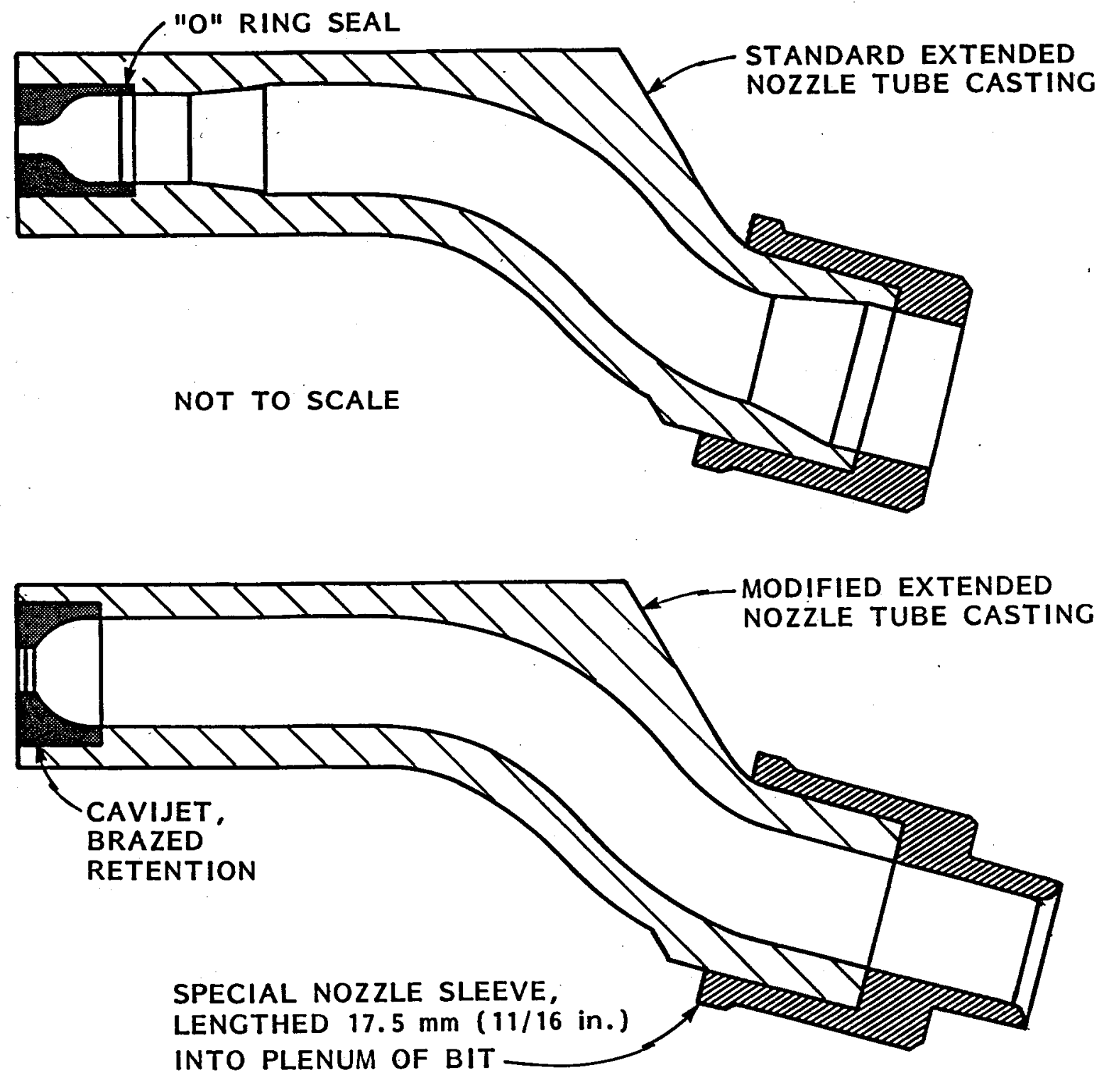

FIGURE 52 - MODIFICATIONS TO TUBES FOR ORGAN PIPE CAVIJET $^{\circledR}$ NOZZLES IN THREE-CONE BIT 
$-64,-$

(0.4 scale), in the HYDRONAUTICS High Pressure Cell (HPC). A nozzle test series using the single 0.4 scale model tube included runs with a water-polymer solution (Kelzan at 1 and 21 bs per bb1) that showed essentially the same resonant modulation characteristics as for pure water. Thus, we had hopes that drilling mud would also behave similarly.

The specified (design) operating ranges for the modified bit were:

$\begin{array}{ll}\text { Formation depth } & 1,830 \text { to } 3,960 \mathrm{~m} \\ & (6,000 \text { to } 13,000 \mathrm{ft}) \\ \text { Mud Weight } & 1 \text { to } 1.43 \mathrm{gm} / \mathrm{cm}^{3} \\ & (8.33 \text { to } 12 \mathrm{ppg}) \\ \text { Bit Pressure Drop } & 9.0 \text { to } 11.7 \mathrm{MPa} \\ & (1,300 \text { to } 1.700 \mathrm{psi}) \\ \text { Flow Rate } & 30.3 \text { to } 37.8 \mathrm{l} / \mathrm{s} \\ & (480 \text { to } 600 \mathrm{gpm})\end{array}$

One specimen of Mancos shale was bored out with each test bit. Twenty-eight drilling runs were made with the standard bit and 24 runs, having similar parameter variations, were made with the modified bit. Water-based $1.14 \mathrm{gm} / \mathrm{cm}^{3}(9.5 \mathrm{ppg})$ mud was used for both tests, but the apparent viscosity was higher for the standard bit test, 26.5 centipoise compared to 15 centipoise for the modified bit. Mud characteristics are summarized in Table 4 (see page 66).

Drilling test parameters were varied as follows:

\section{Parameter}

Bore Hole Pressure $\left(p_{a}\right)$

Simulated Depth
Range

4.8 to $19.3 \mathrm{MPa}$ (700 to 2800 psi)

460 to $1740 \mathrm{~m}$ (1500 to $5700 \mathrm{ft}$ ) 
Parameter (cont.)

Nozzle Pressure Drop ( $(\mathrm{p})$

Mud Flow (Q)

Hydraulic Horsepower/in. ${ }^{2}$

Weight on Bit (WOB)

Rotative Speed
Range (cont.)

2.4 to $12.1 \mathrm{MPa}$

(500 to $1750 \mathrm{psi}$ )

22.7 to $34.7 \mathrm{\ell} / \mathrm{s}$

(360 to $550 \mathrm{gpm}$ )

0.7 to $4.0 \mathrm{HSI}$

44 to $156 \mathrm{kN}$

$(10,000$ to $3.5,000 \mathrm{lb})$

8 to 140 RPM

The dependent measured parameters were drilling rate (ROP) and resonant pressure modulation frequency and amplitude. Before commencing drilling tests with the modified bit, jetting tests were run with water and then drilling mud to examine the pressure fluctuations.

\subsection{Pressure Fluctuation Test Results}

One of the primary objectives of the full-scale tests at DRL was to determine what changes, if any, would arise in the resonance behavior of self-resonating cavitating nozzles when the working fluid was changed from water to mud. Hence, one piezoelectric transducer was mounted in the feed pipe to the bit and a second in the return flow line, ahead of the choke valve used to maintain borehole pressure. The two transducers served to monitor pressure fluctuations in the drilling fluid. High pass filters ( $1.0 \mathrm{kHz}$ corner) were used to eliminate any low frequency, pump-induced, pressure fluctuations from the transducer signals which were displayed on a dual channel oscilloscope. Typical photographs of the oscilloscope traces are reproduced in Figures 53 and 54. In each case, the upper trace 
$-66-$

\section{TABLE 4}

MUD PROPERTIES FOR TESTS WITH

$31 \mathrm{~cm}\left(12 \frac{1}{4}-\mathrm{in}.\right)$ THREE-CONE BITS

\begin{tabular}{|c|c|c|}
\hline PARAYETER & $\begin{array}{c}\text { Tests With } \\
\text { Standard Nozzles }\end{array}$ & $\begin{array}{c}\text { Tests with } \\
\text { ORGAN-PIPE CAVIJET } \\
\text { Nozzles }\end{array}$ \\
\hline $\begin{array}{l}\text { Date } \\
\text { Time } \\
\text { Test Temp }{ }^{\circ} \mathrm{C}\left({ }^{\circ} \mathrm{F}\right)\end{array}$ & $\begin{array}{l}12 / 22 / 81 \\
3: 00 \mathrm{PM} \\
22.2(72)\end{array}$ & $\begin{array}{c}1 / 7 / 82 \\
6: 40 \mathrm{PM} \\
38.3(101)\end{array}$ \\
\hline $\begin{array}{r}\text { Rheology } \\
600 \text { RPM } \\
300 \text { RPM } \\
200 \text { RPM } \\
100 \text { RPM } \\
6 \text { RPM } \\
3 \text { RPM } \\
\end{array}$ & $\begin{array}{c}\text { Shear Stress, } 1 \mathrm{~b} / 100 \mathrm{ft}^{2} \\
53 \\
35 \\
29 \\
20 \\
5 \\
3\end{array}$ & $\begin{array}{c}\text { Shear Stress, } 1 \mathrm{~b} / 100 \mathrm{ft}^{2} \\
30 \\
19 \\
13 \\
10 \\
2 \\
1\end{array}$ \\
\hline 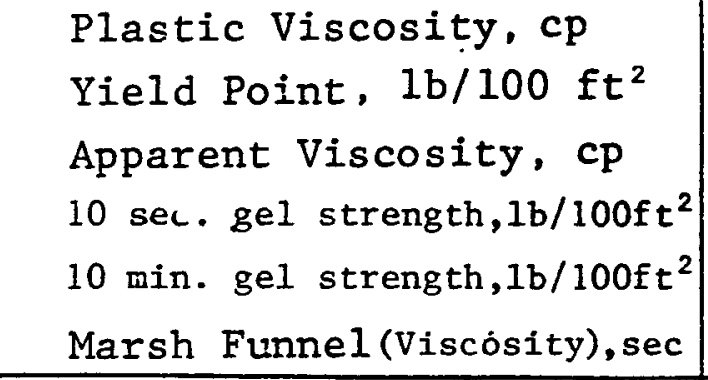 & $\begin{array}{l}18 \\
17 \\
26.5 \\
6 \\
10 \\
45\end{array}$ & $\begin{array}{r}11 \\
8 \\
15 \\
1 \\
2 \\
--\end{array}$ \\
\hline $\begin{array}{l}\frac{\text { Filtration }}{\text { API Filtration, ml/30 min. }} \\
\text { HTHP Filtration } \\
\text { pH }\end{array}$ & $\begin{array}{l}11.2 \\
-- \\
10.0\end{array}$ & $\begin{array}{c}8.9 \\
-- \\
7.9\end{array}$ \\
\hline $\begin{array}{l}\text { Retorts } \\
\% \text { Sand } \\
\% \text { Water } \\
\% \text { Solids } \\
\% \text { Oils }\end{array}$ & $\begin{array}{c}1 / 2 \\
93 \\
7 \\
-\cdots\end{array}$ & $\begin{array}{l}1 / 2 \\
93 \\
7 \\
--\end{array}$ \\
\hline $\begin{array}{l}\frac{\text { Mud }}{\text { Base }} \\
\text { Density, gm/ } \mathrm{cm}^{3} \text { (ppg) }\end{array}$ & $\begin{array}{c}\text { Water } \\
1.14(9.5)\end{array}$ & $\begin{array}{c}\text { Water } \\
1.14(9.5)\end{array}$ \\
\hline
\end{tabular}




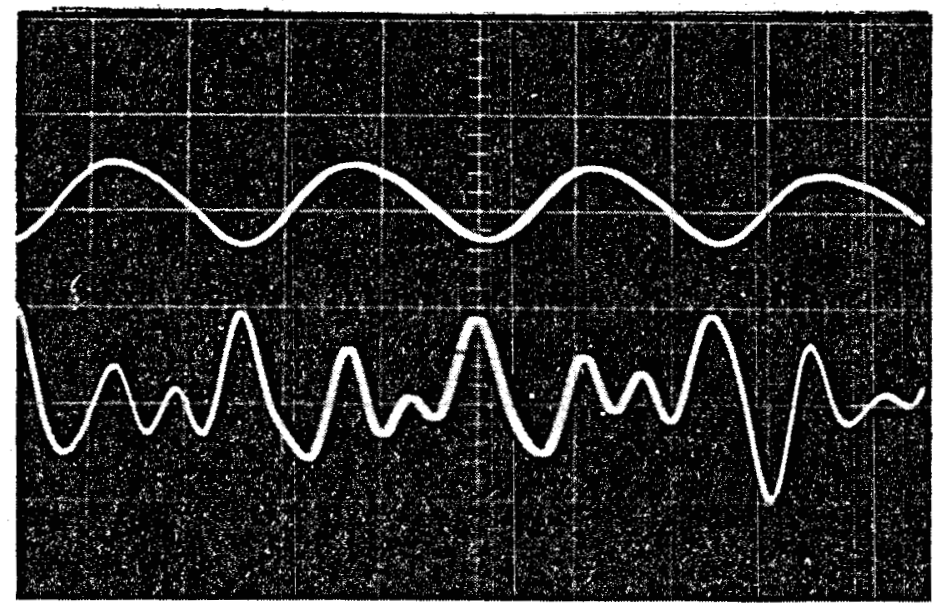

a. $M=0.069$

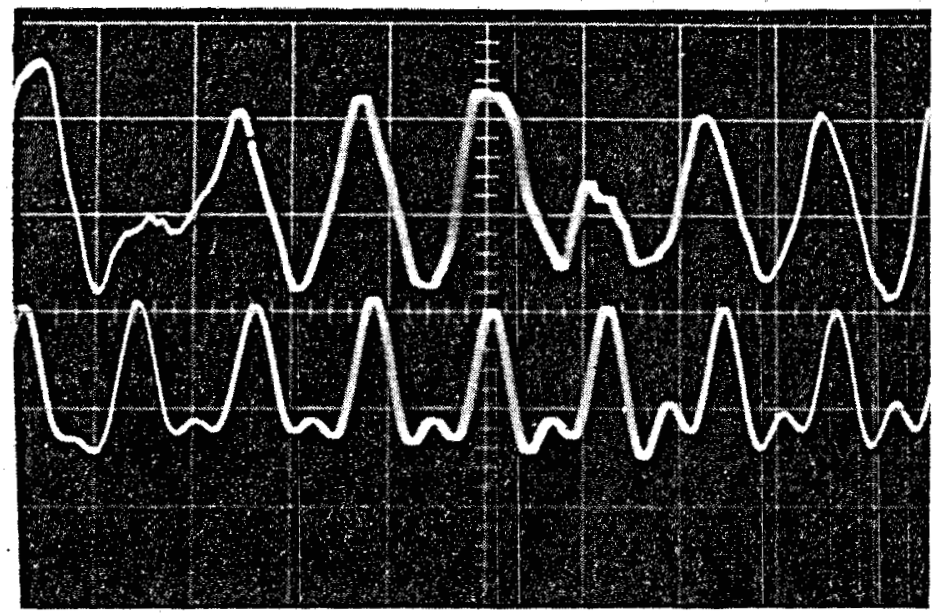

FEED PIPE, $P_{1}^{\prime}$

b. $M=0.086$

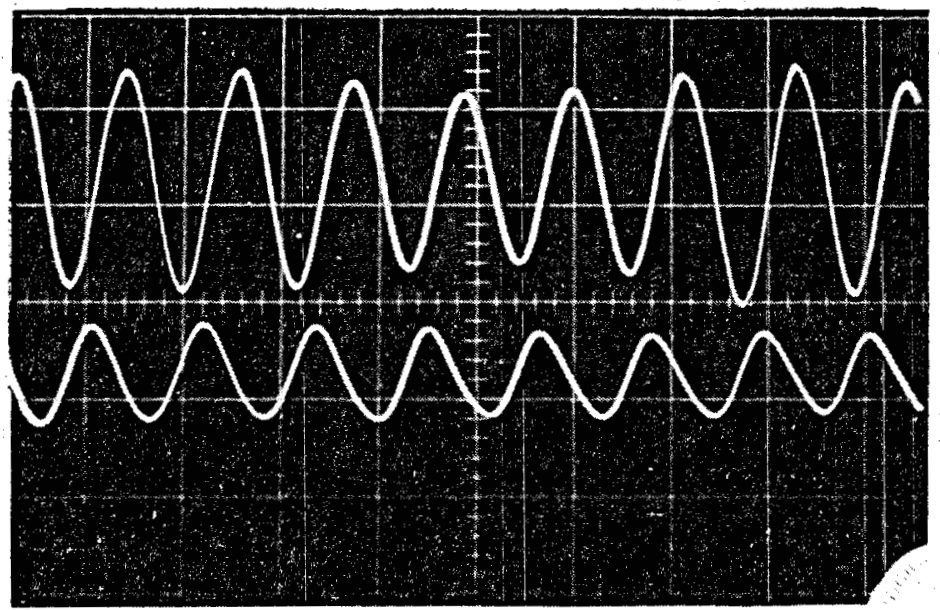

c. $M=0.095$

CHOKE LINE, $p_{2}^{\prime}$

\section{NOTE: Amplitudes are at \\ Amplitudes are at
Arbitrary scales}




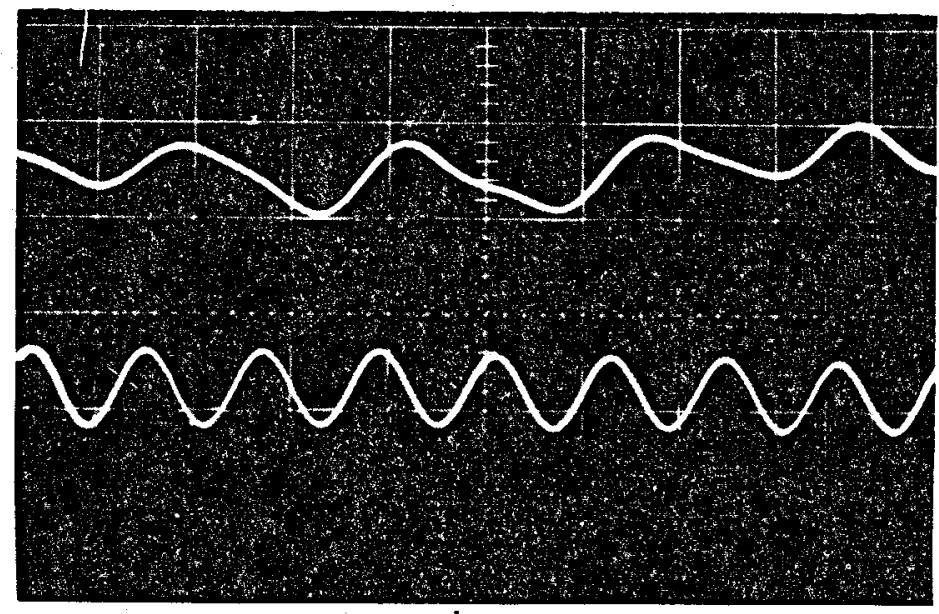

a. $M=0.067$

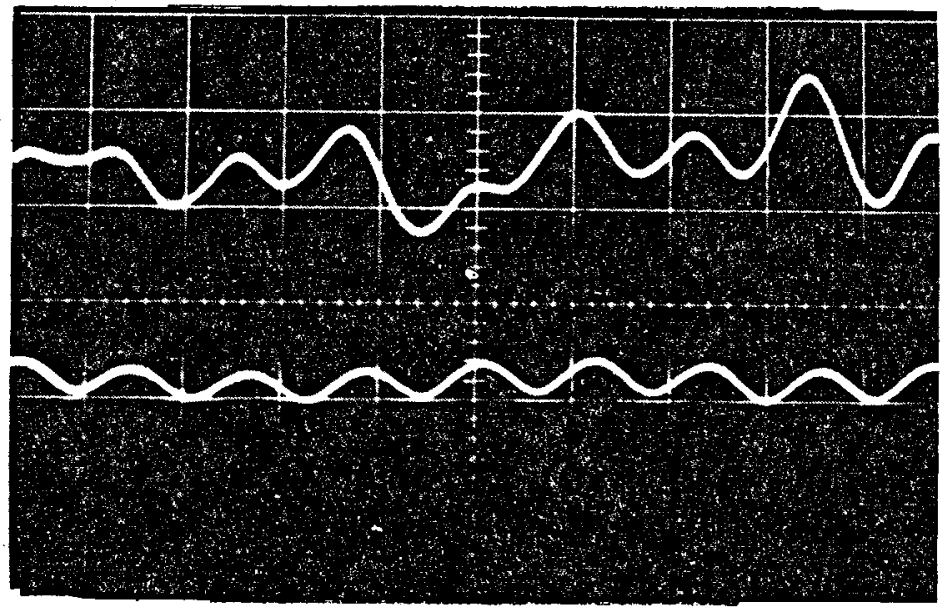

FEED PIPE, $p_{1}^{\prime}$

b. $M=0.076$

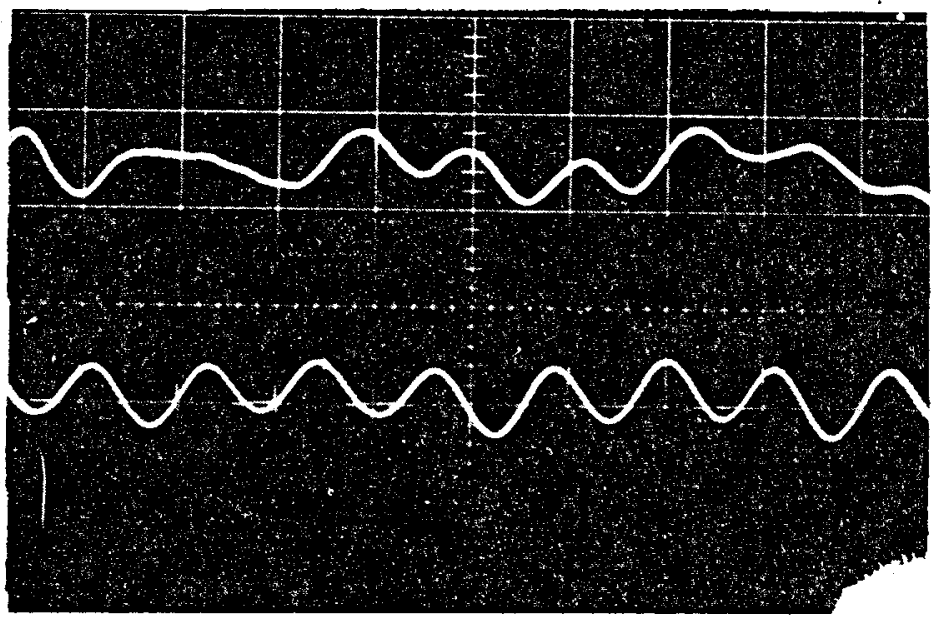

NOTE: Amplitudes are at

Arbitrary Scale

c. $M=0.094$

CHOKE LINE, $p_{2}^{\prime}$

FIGURE 54 - PRESSURE MODULATIONS IN $1.14 \mathrm{gm} / \mathrm{cm}^{3}(9.5 \mathrm{ppg})$ MUD 
is for the feed pipe transducer and the lower trace is the simultaneous choke line transducer output.

The two transducers were located several wavelengths upstream and downstream from the source (drill bit), and the acoustic transmission characteristics of the piping network are very complex, so these signals do not give an exact representation of the modulation of the jets. For example, compare the pair of traces in Figure 53a. The feed line pressure showed a nearly pure $4 \mathrm{kHz}$ sine wave whereas the choke line had an additional $12 \mathrm{kHz}$ component. The signals in Figure 53c, however, both had comparable $8.6 \mathrm{kHz}$ frequencies with some slight amplitude modulation in the choke line trace.

Before commencing drilling tests with the modified bit, a series of pressure fluctuation tests were run; first with water and then with drilling mud. The results are sumarized in Figure 55. Here the measured rms amplitudes of the pressure fluctuations, $\mathrm{P}^{\prime}$, were normalized by the pressure drop across the nozzles, $\Delta p$, and plotted as a function of the jet Mach number, $M$. Note, Mach number is proportional to $(\Delta \mathrm{p} / \rho)^{\frac{1}{2}}$, where $\rho$ is the fluid density. Significant differences were observed between the resonance response of this modified ORGAN-PIPE CAVIJET bit with water and with the "standard" DRL $1.14 \mathrm{gm} / \mathrm{cm} \quad(9.5 \mathrm{pPg})$ water-based mud.

7.2.1 Water - With water, the modulations in the feed pipe showed characteristics similar to 40 percent scale models tested earlier at HYDRONAUTICS: a 7 percent first mode peak with $\mathrm{S}_{\mathrm{d}}=$ $0.41(4 \mathrm{kHz}$ at $M=0.062$ and $\sigma=1.0)$ and an 8 percent second mode peak with $S_{d}=0.57(8.6 \mathrm{kHz}$ at $M=0.096$ and $\sigma=1.5)$. Much higher amplitudes were experienced with the scale model tests (up to 35 percent in the feed pipe at $M=0.095$ ), but in 
view of the geometric differences with the full scale bit configuration, such disparity was not unexpected.

The pressure modulations in the choke line were lower, with a peak amplitude of $\mathrm{p}^{\prime} / \Delta \mathrm{p}=2$ percent at $M=0.090$. Amplitudes measured in the choke were not affected greatly by cavitation number for $\sigma \geq 1$. Note: open symbols denote tests for which $\Delta \mathrm{p}+\mathrm{p}_{\mathrm{a}}=25.5 \mathrm{MPa}$ ( $3700 \mathrm{psi}$ ), the maximum pump capability; $\sigma=I$ for filled symbols.

Modulations in the feed pipe were reduced at $\sigma=1$ compared to higher $\sigma$ results. This result is consistent with the 40 percent scale model nozzle tests.

7.2.2 Drilling Mud - The measured amplitude modulations were reduced with the drilling fluid, $1.14 \mathrm{gm} / \mathrm{cm}^{3}(9.5 \mathrm{ppg})$ water -based mud. The maximum amplitude was only 0.5 percent for a second mode peak with $\mathrm{S}_{\mathrm{d}} \approx 0.73(8.5 \mathrm{kHz}$ at $\mathrm{M}=0.073)$. Resonant frequencies were the same with mud and water, hence, sound speed was apparently not affected by the mud additives.

Unlike the results with water, amplitudes in the choke generally exceeded corresponding values in the feedpipe; and amplitudes measured at $\sigma=1$ were found to be larger than for higher cavitation numbers. Furthermore, the frequency $(\approx 8 \mathrm{kHz})$ of the modulation in the choke was more discrete and regular than in the feedpipe (see oscillograph traces, Figure 54).

Resonance measurements for the choke line made with the standard $31 \mathrm{~cm}\left(12 \frac{2}{4}-i n.\right)$ three-cone bit showed very low amplitude resonance peaks with a frequency of about $2 \mathrm{kHz}$. The peak amplitude with standard nozzles was only about $p^{\prime} / \Delta p=0.1$ percent.

During drilling tests, the feed line transducer was disconnected to allow feed pipe shaft rotation. Pressure fluctuations in the choke line were still monitored, however. Generally, 
the measured modulation amplitude in the choke line increased when the bit was lowered to the bottom of the hole; the peak modulation was found to be $\mathrm{p}^{\prime} / \Delta \mathrm{p}=1.6$ percent at $\Delta \mathrm{p}=6.9 \mathrm{MPa}$ (1000 psi) and $p_{a}=8.6 \mathrm{MPa}$ (1250 psi).

\subsection{Bit Hydraulics Analysis}

During the planning for these tests at DRL, the results of laboratory studies at HYDRONAUTICS were used to select the orifice diameter for the ORGAN-PIPE CAVIJET nozzles, so that equivalent hydraulic power would be delivered across both the standard and the modified bits (when each was operated at the same pressure drop, $\Delta \mathrm{p})$. It had been determined that the discharge coefficient, $C_{D}$, of a conventional CAVIJET nozzle was about 0.67 , versus about 0.95 for a standard SMITH drill bit nozzle. Our tests at HYDRONAUTICS had also shown that increases in the pressure fluctuations, $p^{\prime} / \Delta p$, of self-resonating CAVIJET nozzles caused increases in their discharge coefficient. Thus, based on these results, it was estimated that an orifice diameter, $d=9.5 \mathrm{~mm}$ (12/32 in.) for the ORGAN-PIPE CAVIJET nozzles should provide the desired comparable flow to that of the $8.7 \mathrm{~mm}$ (11/32 in.) standard SMITH nozzles. This did not occur. For instance, at a $\Delta \mathrm{p}=11.7 \mathrm{MPa}(1,700 \mathrm{psi})$, with $\mathrm{P}_{\mathrm{a}}=10.3 \mathrm{MPa}(1,500 \mathrm{psi})$ the total flow across the standard bit was $32.8 \mathrm{2} / \mathrm{s}$ (520 $\mathrm{gpm}$ ), or about 12 percent more hydraulic power than represented by the $29.3 \mathrm{l} / \mathrm{s}$ (464 $\mathrm{gPm}$ ) for the bit containing the ORGAN-PIPE CAVIJET nozzles. As discussed below, this discrepency in hydraulic power was certainly a contributing factor to the slower rates of penetration observed for the modified bit under many of the tested operating conditions.

To examine more closely the actual flow characteristics of these bits, an analysis was made of the partioning of the flow between the nozzles in the extended tubes and center venturi 


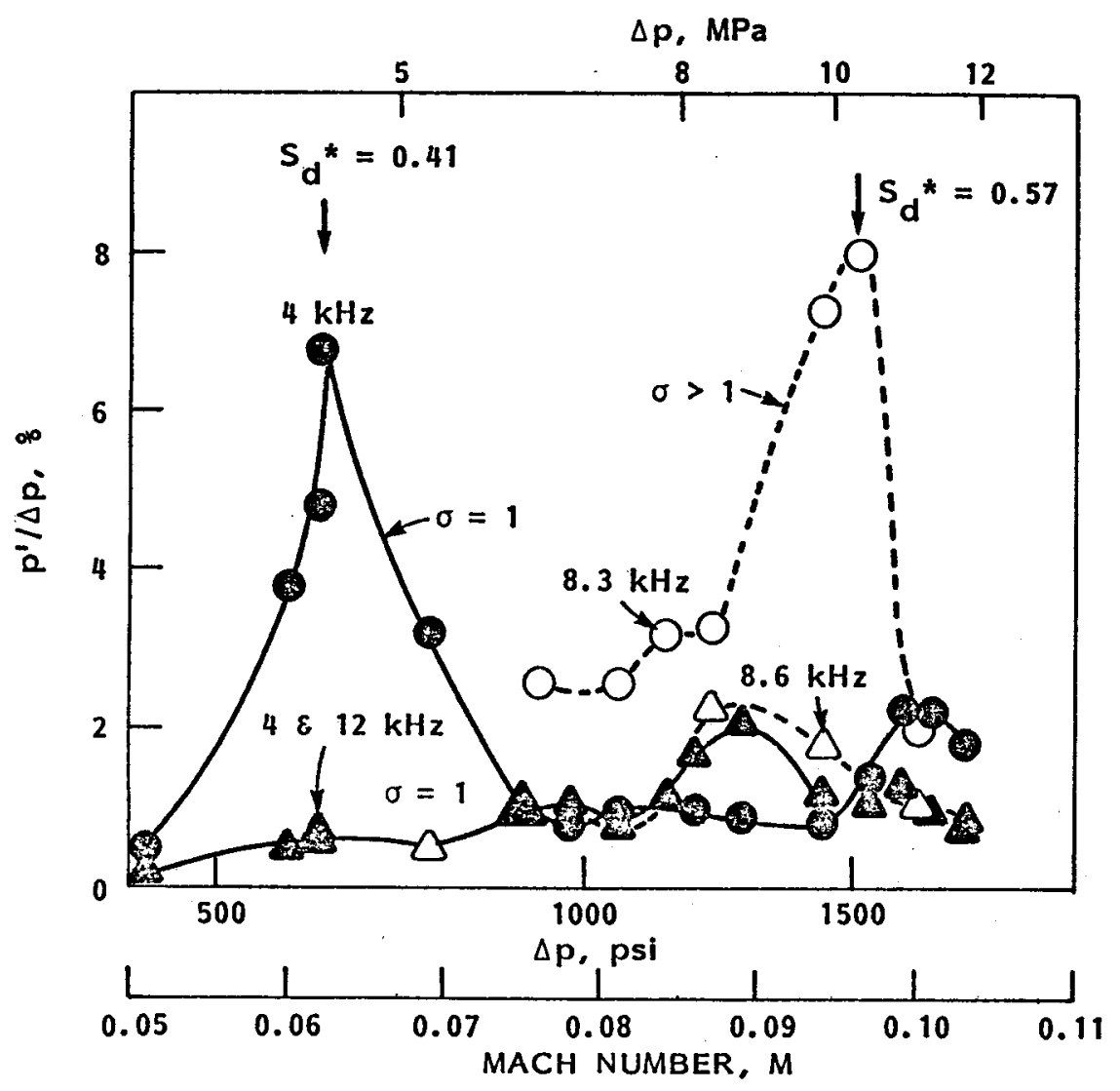

a. TESTS WITH WATER

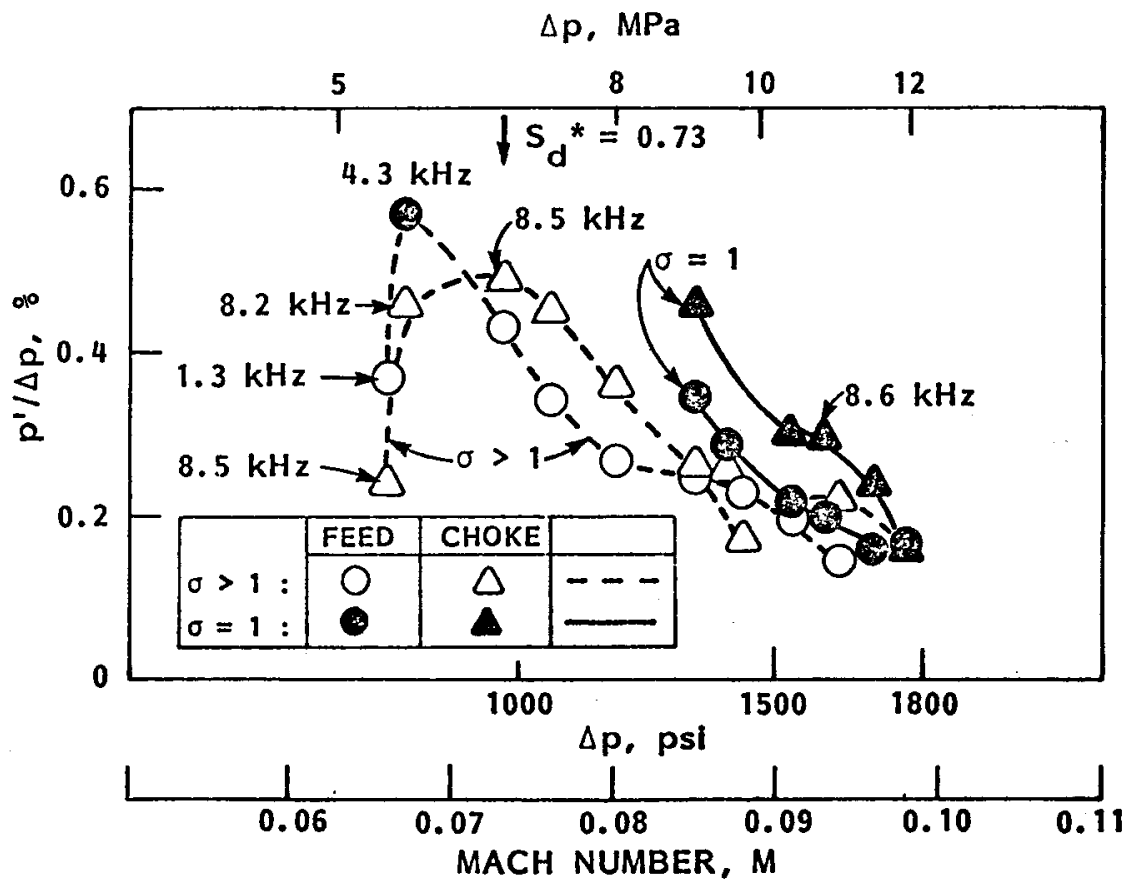

b. TESTS WITH $1.14 \mathrm{gm} / \mathrm{cm}^{3}(9.5 \mathrm{ppg})$ WATER-BASED MUD

FICURE 55 - PRESSURE MODULATION AMPLITUDES IN WATER AND MUD. Tests at DRL, Jan 7, 1982, with modified. $31 \mathrm{~cm}\left(12 \frac{1}{\mathrm{in}}\right.$.) three-cone bit. 
nozzles. It was known (19) that the $C_{D}$ for this nozzle was strongly influenced by cavitation effects; and experiments by Pearce and Lichtarowicz (20) with similar, internally-cavitating, nozzles demonstrated a $C_{D}$ dependence on a "cavitation index", $\sqrt{1+\sigma}$. As indicated by the solid curve fitted through the experimental data in Figure 56, this center venturi nozzleihad a $C_{D}$ behavior comparable to the nozzles studied in Reference 20 . This fitted curve was based on assigning the discharge coefficients cited above, namely $C_{D}=0.95$ for the SMITH nozzles and $C_{D}=$ 0.67 for the CAVIJET nozzles. This meant that for an "equivalent" diameter of $8.7 \mathrm{~mm}(11 / 32 \mathrm{in.})$, the CAVIJET nozzles had a $C_{D}=$ 0.80 , or only 84 percent $(0.80 / 0.95)$ of the standard nozzles. In effect, the modified bit therefore had nozzles which had a flow equivalence of $7.9 \mathrm{~mm}$ (10/32 in.) nozzles instead of the planned $8.7 \mathrm{~mm}$ (11/32 in.) nozzle equivalence. This condition was caused, at least in part, by the small amplitudes of the pressure fluctuations achieved with the $1.14 \mathrm{gm} / \mathrm{cm}^{3}$ (9.5 ppg) drilling mud.

In the earlier laboratory tests with a 40 percent scale ORGAN-PIPE CAVIJET nozzle, the $C_{D}$ was seen to increase proportionally with increases in the pressure modulation ratio, $\mathrm{p}^{\prime} / \Delta \mathrm{p}$, as roughly:

$$
C_{D} \propto 1+\frac{1}{2}\left(p^{\prime} / \Delta p\right)
$$

A comparable trend was observed in the measured flow for the modified bit in the DRL tests, as seen in Figure 57 . These data for the ORGAN-PIPE CAVIJET nozzles were derived by subtracting the flow through the center venturi nozzle in this bit as indicated by Figure 56 . If the least square fit line in Figure 57 is extrapolated to a pressure fluctuation ratio. 


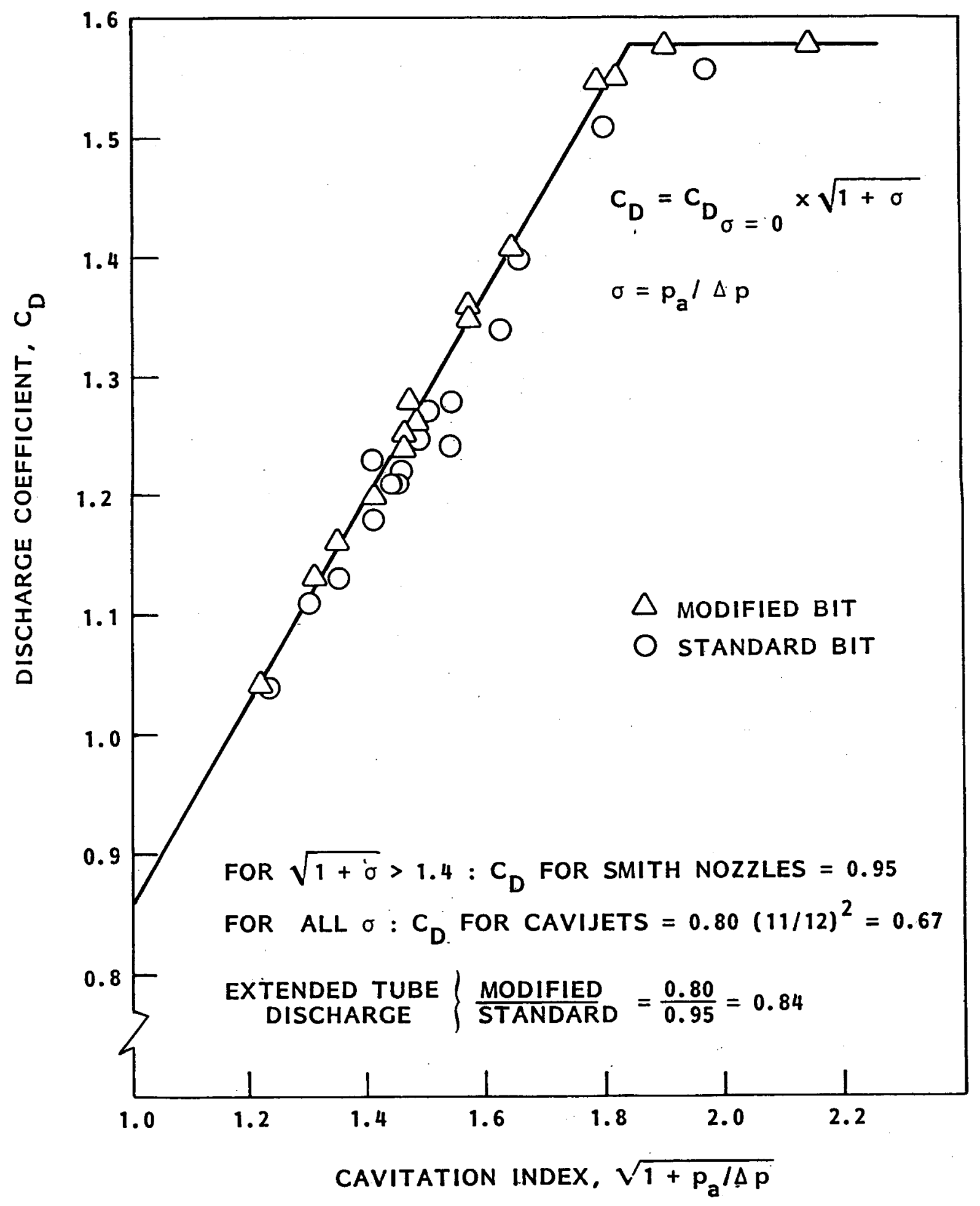

FIGURE 56 - DISCHARGE COEFFICIENT FOR CENTER VENTURI JET 


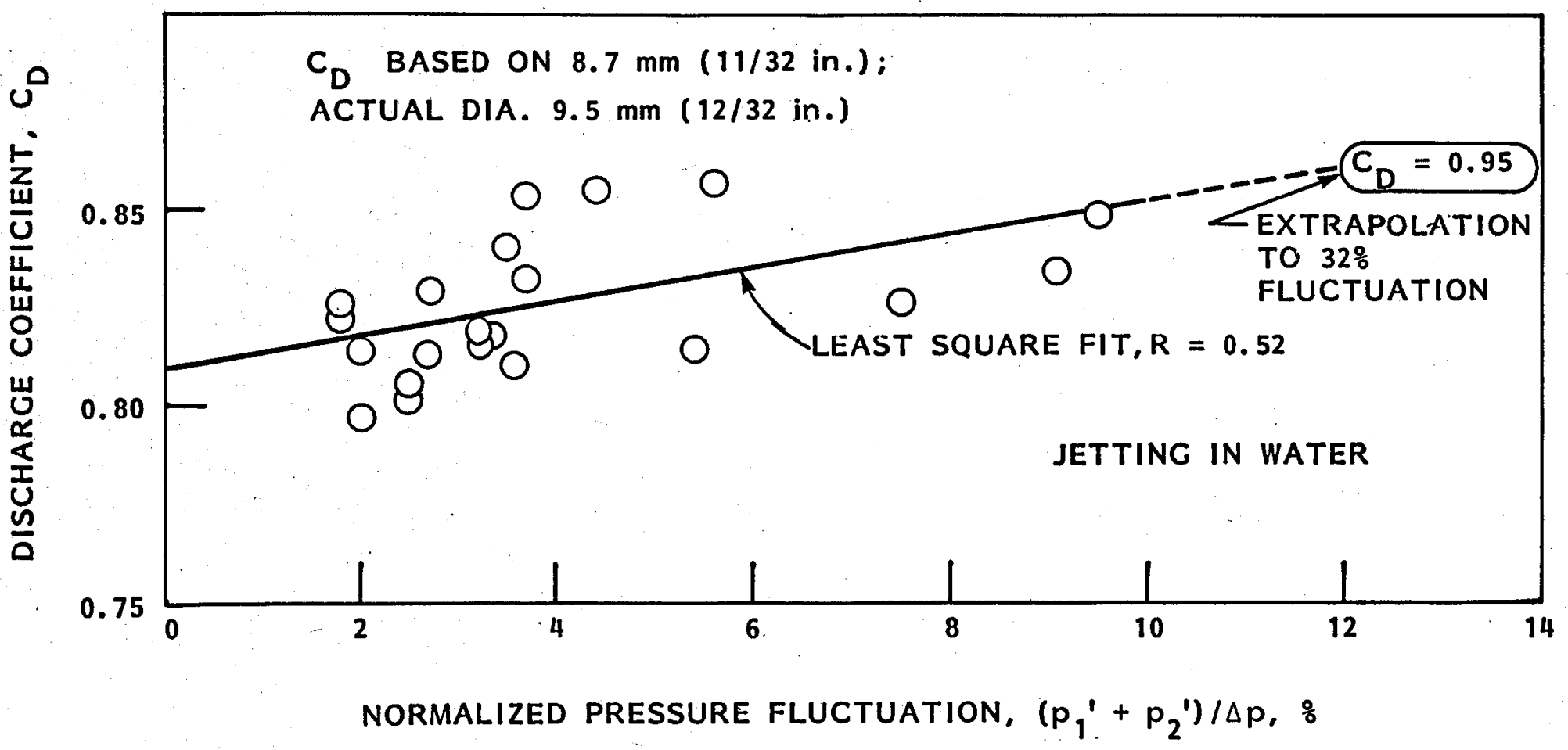

FIGURE 57 - LEAST SQUARE FIT TO MODIFIED BIT DISCHARGE THROUGH EXTENDED TUBE NOZZLES 
$\left(p_{1}^{\prime}+p_{2}^{\prime}\right) / \Delta p=32$ percent, (where $p_{1}^{\prime}$ and $p_{2}^{\prime}$ are, respectively, the pressure fluctuations measured in the feed pipe and choke line), then a $C_{D}=0.95$ would be achieved. This discharge coefficient is equivalent to that of the SMITH nozzles. This suggests that if pressure fluctuations comparable to those for the laboratoryscale nozzles had been obtained with the modified bit in the DRL tests, then the desired flows would have been achieved for this bit. Note, the scaling of $\mathrm{p}_{1}^{\prime}, \mathrm{p}_{2}^{\prime}$ and $\mathrm{p}^{\prime}$ (on the 40 percent nozzle model) is not known; their relative amplitudes are complex functions of the specific geometries and their acoustic response.

\subsection{Drilling Tests Results}

Variations of bore hole pressure, nozzle pressure, weight on bit and RPM were duplicated in tests with both bits. The effects of these parameters on the rate of penetration were as follows.

7.4.1 WOB - Figure 58 shows a linear increase in ROP with weight on bit up to $155.7 \mathrm{kN}(35,000 \mathrm{lb})$. The rate of penetration for the modified bit was 84 percent of the baseline ROP for the standard bit.

7.4.2 RPM - Figure 59 suggests that bottom hole cleaning with the modified bit is being limited by the lower flow that was achieved: $29.3 \mathrm{l} / \mathrm{s}$ (464 gpm) compared to $32.8 \mathrm{l} / \mathrm{s}$ (520 gpm) for the baseline bit. The ROP is levelling off above 80 RPM whereas the standard bit ROP was nearly linear up to 120 RPM.

7.4.3 $\mathrm{p}_{a}$ and $\Delta \mathrm{p}$ - Figure 60 shows the ratio of drilling rate of the modified bit to drilling rate of the baseline or standard bit. It ranged from a low of $0.84(\Delta \mathrm{p}=10.3 \mathrm{MPa}$ (1500 psi), $\left.\mathrm{p}_{\mathrm{a}} \simeq 11.7 \mathrm{MPa}(1700 \mathrm{psi})\right)$ to a high of $1.09(\Delta \mathrm{p}=6.9 \mathrm{MPa}(1000$ psi), $\left.\mathrm{p}_{\mathrm{a}}=8.3 \mathrm{MPa}(1200 \mathrm{psi})\right)$. The modified bit relative drilling performance seems to be correlated with the modulation amplitude 


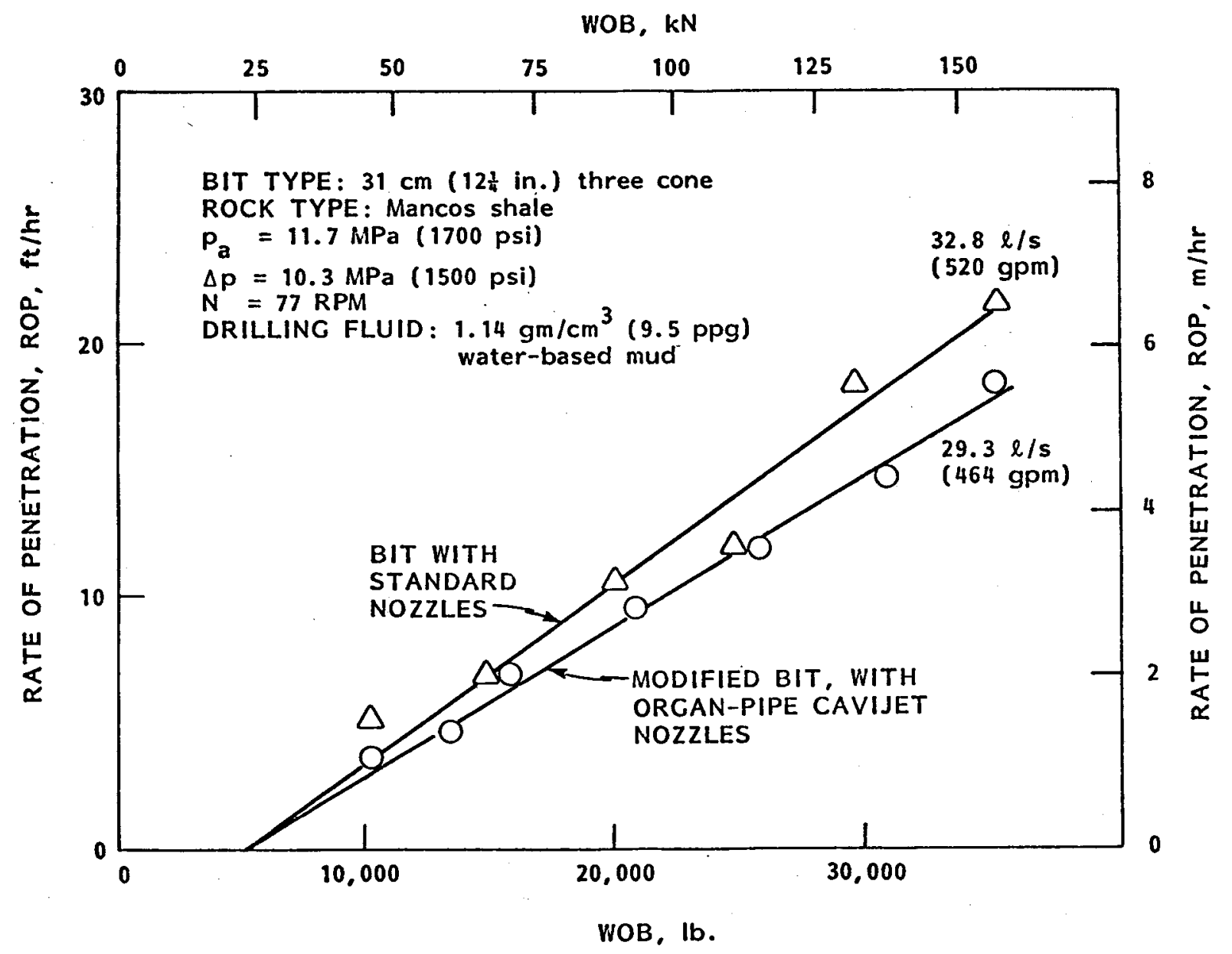

FIGURE 58 - EFFECT OF WEIGHT ON BIT ON DRILLING RATE 


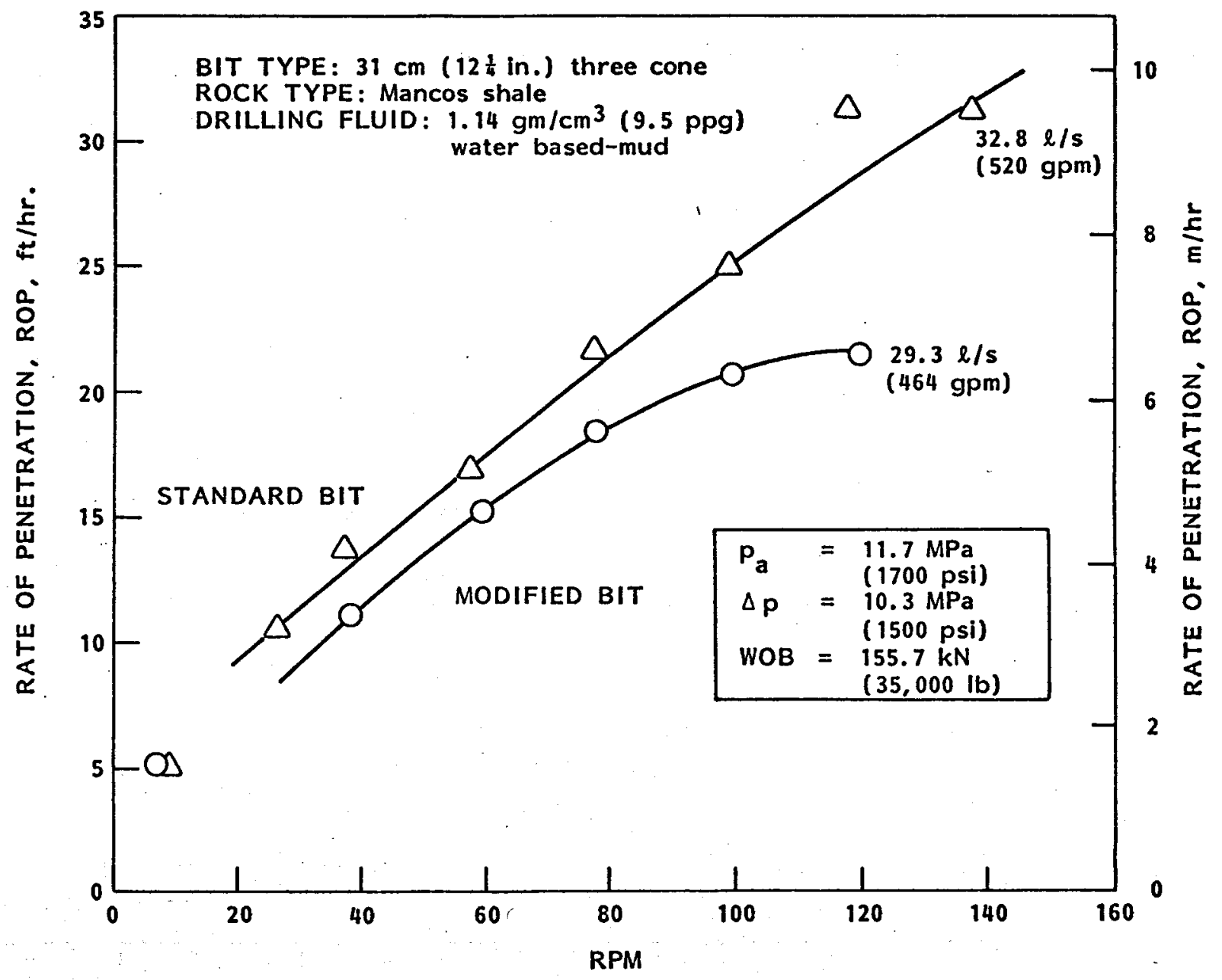

FIGURE 59 - EFFECT OF RPM ON DRILLING RATE 


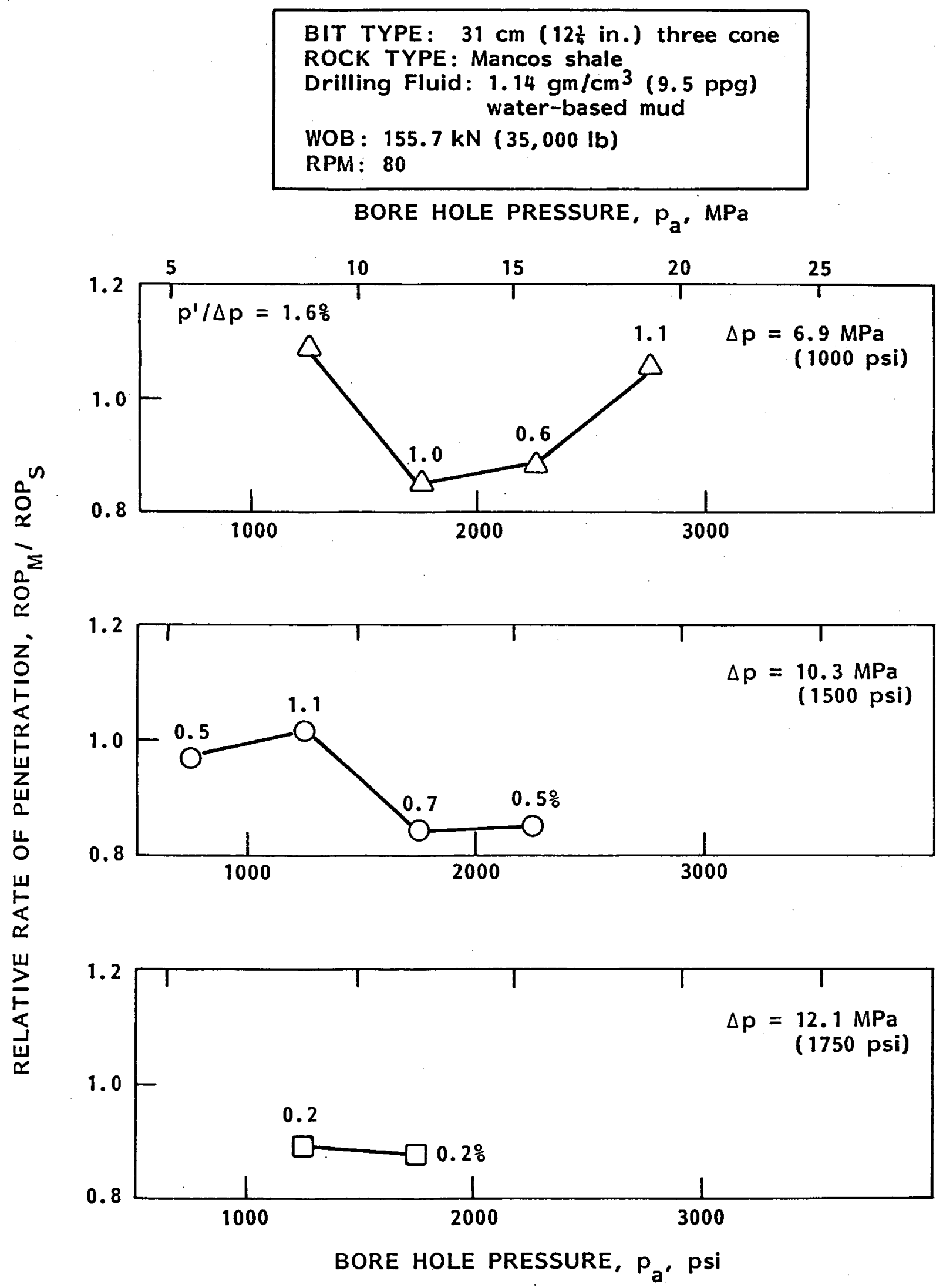

FIGURE 60 - RELATIVE DRILLING PERFORMANCE VS. BORE HOLE PRESSURE 
that was achieved in the drilling mud.

7.4.4 Modulation and Drilling Rate - The relative drilling rate of the modified bit is shown as a function of the pressure modulation amplitude in Figure 61. The line drawn is a least squares fit to the data points; it suggests that a modulation amplitude of 1.2 percent has offset the loss in performance caused by this bit's reduced flow (hydraulic horsepower) relative to the baseline bit.

\subsection{Conclusions from DRL Tests with Three-Cone Bits}

Pressure modulation characteristics of the full scale ORGANPIPE CAVIJET nozzles in water were comparable to those of earlier 40 percent scale models, but measured amplitudes were much lower, presumeably caused by the acoustic response of the complex bit and piping geometry.

Discharge through the modified extended tube nozzles was 16 percent less than for the standard bit. This condition arose from the low modulation levels that were achieved with 1.14 $\mathrm{gm} / \mathrm{cm}^{3}$ (9.5 ppg) drilling mud in the modified design (compared to the 35 percent levels that were achieved in 40 percent scale model tests in water). In the absence of strong pressure fluctuations the actual discharges obtained must be expected to be low.

The Mach number (or Strouhal number) for peak pressure modulation amplitude was significantly changed by mud properties as compared to water. The amplitude was much reduced in mud, while the frequency was unchanged.

At low modulation (under one percent in the cholke line) the modified bit ROP was as much as 16 percent less than the standard bit; presumably caused by the reduced flow noted above. In view of the foregoing remarks this result is to be expected. 


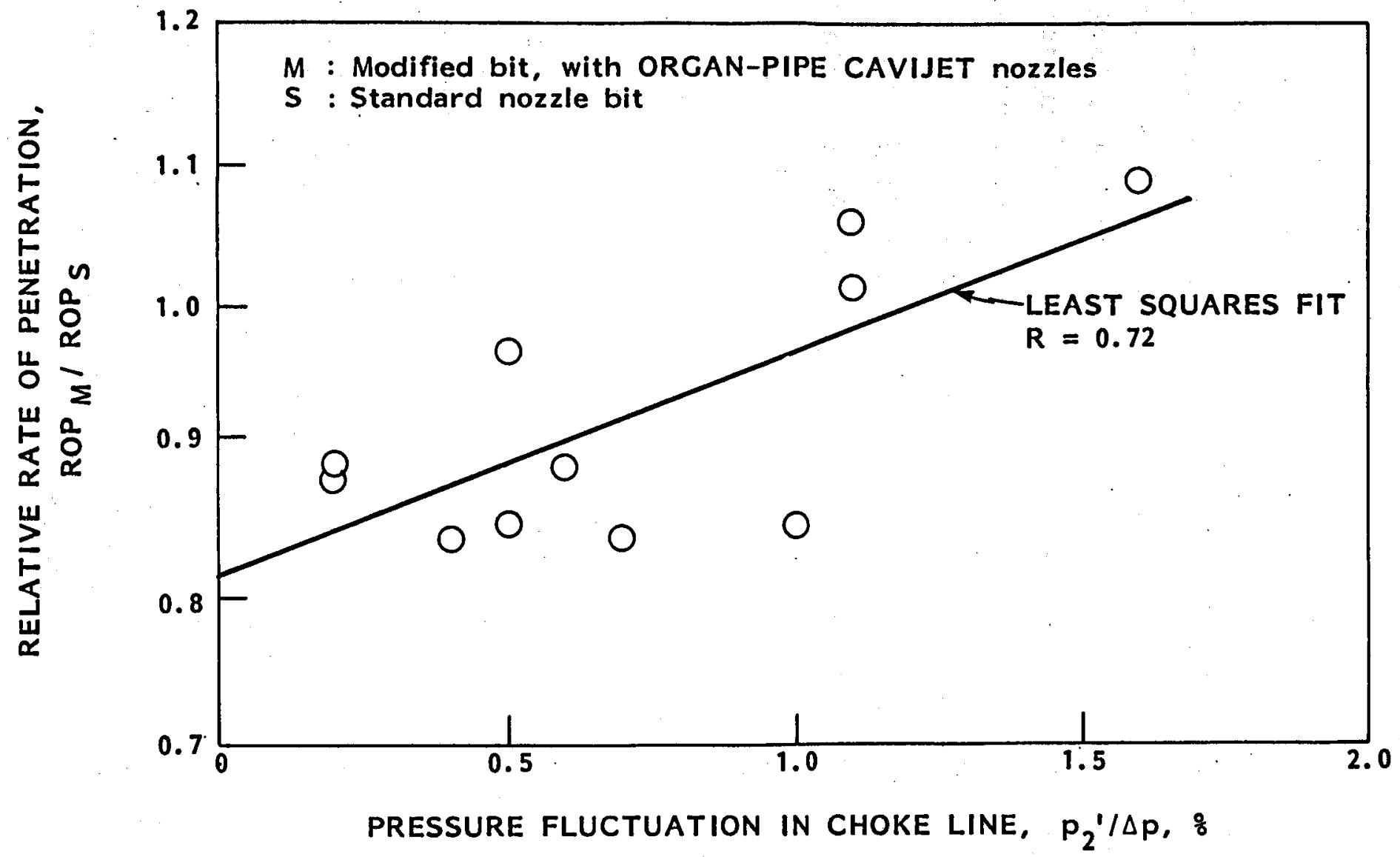

FIGURE 61 - EFFECT OF MODULATION AMPLITUDE ON RELATIVE DRILLING RATE 
If the nozzle system is not strongly resonating, it is no different than the standard bit (if sized on the basis of no resonance to pass the same discharge).

We are encouraged by the drilling rate near $\Delta \mathrm{p} \simeq 6.9 \mathrm{MPa}$ (1000 psi) where the strongest resonance (although still relative1y weak) did occur. There is evidence here that the resonance effect does increase ROP. With only moderate modulation ( 1.1 and 1.6 percent) the modified bit ROP slightly exceeded the standard bit (by 1 to 9 percent), despite the deficit in hydraulic power.

Increased ROP's relative to the standard bit should be realized by optimizing the nozzle orifice design to obtain higher (order 10 percent) modulations with mud at $\Delta \mathrm{p}=10.3 \mathrm{MPa}$ (1500 psi) and increasing the nozzle diameter to achieve comparable flow (but compensating for the effect of higher modulation amplitude).

The DRL tests provided the not-unexpected result that a nozzle designed for optimum resonance in water will provide a different resonating response in drilling mud. We therefore plan, based on these DRL results, to embark upon the same type of systematic developmental effort, in mud, as was successfully conducted to achieve strong resonance in water. Assistance with this effort, in conjunction with anticipated continued support of more basic studies from the DOE, will be provided by the NL Corporation. Use of the mud-test loop at NL's DST (Drilling Systems Technology) laboratory in Houston will be included, in addition to NL/HYCALOG's usual on-going support (see the FOREWORD of this report). After mud-resonating nozzles are developed, bit tests at DRL will be conducted, and field trials of successful bit designs will follow soon thereafter. 


\subsection{CONCLUSIONS AND RECOMMENDATIONS}

A variety of experimental and analytical tasks were performed during this phase of our effort to develop cavitating jets which are suitable for enhancing the performance of deep-hole drill bits. From these efforts a number of specific conclusions have been drawn - these are listed in each of the papers (Appendices $A, B$, and $C$ ) which form a part of this report - and are summarized here.

1. Several self-resonating nozzles types, the PULSER, PULSERFED, and ORGAN-PIPE CAVIJET designs produce passive oscillations which (a) cause the jet to structure into discrete ring vortices, and thus (b) provide nozzle systems which have incipient cavitation numbers two to six times higher than obtained with either conventional CAVIJET nozzles or typical drill bit nozzles,

2. In rock cutting trials, higher incipient cavitation numbers have been correlated with greater jet erosivity.

3. The ORGAN-PIPE CAVIJET, or STRATOJET system is adaptable to the physical restraints of existing bits, and is the most promising concept for future bit designs.

4. STRATOJET nozzle systems will clean chips from a simulated hole bottom at a nozzle pressure only about 25 percent of that required for standard nozzle systems.

5. Self-resonating structured jets can be achieved either with or without cavitation. Therefore, at hole depths below where cavitation is present, a STRATOJET nozzle may still contribute to improved bottom hole cleaning.

In addition to these specific conclusions, certain general remarks can be made:

1. Several systematic empirical and analytical tools were 
developed which have allowed the design of STRATOJET nozzles that provide high amplitude pressure fluctuations under deep-hole conditions, with water as the working fluid.

2. Additional research and development is required to develop STRATOJET nozzles that are;

a. Compatible with various types of drilling muds, and

b. Capable of performing within various specific drill bit designs,

To reach the ultimate objective of this program, namely cavitating nozzle systems which can be utilized in each of the myriad conditions of fluid properties, operating pressures, and bit configurations encountered when drilling geothermal or petroleum wells, the studies described here should be continued, seeking answers to the following questions:

1. What are the fluid properties of drilling mud which affect the self-resonance phenomena, and how can STRATOJET nozzles be designed for optimum performance in these non-Newtonian fluids?

2. What are the detailed flow field characteristics of self-resonating cavitating jets, and how can improved understanding of the jet fluid dynamics be used to develop improved bit nozzle systems for both existing and higher pressure drilling equipment?

3. How can self-resonating jets be adapted to improve the hole-bottom cleaning action? 


\section{REFERENCES}

1. Conn, A. F., Johnson, V. E., Jr., Liu, H-L., and Frederick, G. S., "Evaluation of CAVIJET Cavitating Jets for Deep-Hole Rock Cutting," HYDRONAUTICS, Incorporated Technical Report 7821-1, August 1979 (Sandia Report No. SAND81-7067, May 1981).

2. Johnson, V. E., Jr., Lindenmuth, W. T., Conn, A. F., and Frederick, G. S., "Feasibility Study of Tuned-Resonator, Pulsating Cavitating Water Jet for Deep-Hole Drilling," HYDRONAUTICS, Incorporated Technical Report 8001-1, May 1981 (Sandia Report No. SAND81-7126, August 1981).

3. Johnson, V. E., Jr., Chahine, G. I., Lindenmuth, W. T., Conn, A. F., Frederick, G. S., and Giacchino, G. J., Jr., "Cavitating and Structured Jets for Mechanical Bits to Increase Drilling Rate," ASME Paper No. 82-Pet-13, presented at the Energy Technology Conference and Exhibition, New Orleans, March 7-11, 1982.

4. Johnson, V. E., Jr., Conn, A. F., Lindennuth, W. T., Chahine, G. L., and Frederick, G. S. "Self-Resonating Cavitating Jets," Proceedings of the Sixth International Symposium on Jet Cutting Technology, sponsored by the BHRA, Surrey, England, Paper A1, April 1982.

5. Johnson, V. E, , Jr., Chahine, E. L., Lindenmuth, W. T., Conn, A. F., Frederick, G. S., and Giacchino, G. J., Jr., "The Development of Structured Cavitating Jets for Deep-Hole Bits," SPE Paper No. 11060, Society of Petroleum Engineers of AIME, presented at the 57 th Annual SPE Fall Conference, New Orleans, LA, September 26-29, 1982.

6. Johnson, V. E., Jr., et al, "Tunneling, Fracturing, Drilling and Mining with High Speed Water Jets Utilizing Cavitation Damage," First Int'1. Sympos. on Jet Cutting Technology, Coventry, England, Paper A3, 1972.

7. Conn, A. F. and Rudy, S. L., "Parameters for a Ship Hull Cleaning system Using the CAVIJET CAvitating Water Jet Method, HYDRONAUTICS, Incorporated Technical Report 7510-1, July 1975.

8. Conn, A, F, , Cohen, S, H., and Frederick, G, S, "Evaluation of Bilge Ciéaning with CAVIJET® Cavitating Jet Nozzles," HYDRONAUTICS, Incorporated Technical Report 8018-1, December 1980 . 
9. Conn, A. F. and Frederick, G. S., "Reducing/Separating Recoverable Demolition Wastes with Cavitating Water Jets," HYDRONAUTICS, Incorporated Technical Report 8226-1, March 1982 .

10. Batchelor, G. K., "An Introduction to Fluid Dynamics," Cambridge University Press, London, 1967.

11. El-Ramly, Z., "Aircraft Trailing Vortices, A Survey of the Problem," Carleton University, Faculty of Engineering Technical Report No. ME/A72-1, Ottawa, Canada, November 1972 .

12. Mason, W. H. and Marchman, J. F., "The Farfield Structure of Aircraft hake Turbulence," AIAA Paper 72-40, San Diego, CA, January 1972.

13. Johnson, V. E., Jr, and Goodman, A,, "The HYDRONAUTICS Variable-Pressure, Free-Surface, High-Speed Channe1," Cavitation Research Facilities and Techniques, published by the American Society of Mechanical Engineers, pp. 49$59,1964$.

14. Wylie, E. B. and Streeter, V. L., "Fluid Transients," NicGraw Hill International Book Company, New York, 1978.

15. Kinsler, L. E. and Frey, A. R., "Fundamentals of Acoustics," 2nd ed., John Wiley \& Sons, Inc., New York, 1962.

16. Nebeker, E. B. and Rodriguez, S. E., "Percussive Water Jets for Rapid Excavation," and "Development of Percussive Water Jets," Scientific Associates Technical Reports, 1973, and May 1979.

17. Maurer, W. C., "Bit-Tooth Penetration Under Simulated Borehole Conditions," AIME Iransactions, Vol. 234, Pp. I-1433 to $I-1442,1965$.

18. Tibbitts, G. A., et al,, "The Effects of Bit Hydraulics on Full-Scale Laboratory Drilled Shale," Society of Petroleum Engineers of AIME, Preprint No. SPE 8439, presented at the $54 \mathrm{th}$ Annual SPE Fall Meeting, Las Vegas, Nevada, September 23-26, 1979.

19. Baker, W. "Extended Nozzle Bits Require Precise Nozzle Sizing," The Oil and Gas Journal, Pp, 88-97, March 19, 1979. 
20. Pearce, I. D. and Lichtarowicz, A., "Discharge Performance of Long Orifices with Cavitating Flow," Proceedings of the Second Fluid Power Symposium, sponsored by the BiRA, Guilford, England, Paper D2, January 1971. 
APPENDIX A

"Cavitating and Structured Jets for Mechanical Bits to Increase Drilling Rate"

Presented at the

ASME Energy Technology Conference and Exhibition

New Orleans, Louisiana

March 7r-11, 1982 
V.E. JOHNSON, JR.

Q.L. CHAHINE Mem. ASME

\section{W.T. LINDENMUTH}

A.F. CONN Hem. ASME

G.S. FREDERICK HYDRONAUTICS, Incorporated Laurel, Maryland

G.J. GIACCHINO. JR. NL HYCALOG/NL Industries, Inc. Houston, Texas

\section{Cavitating ahd StRuctured Jets for mechanical BITS TO IHCREASE DRILLIHG RATE}

The erosion and cleaning effect of jets is enhanced when the degree of cavitation occurring on or near the bottom of the hole is increased. Self-excited, acoustically resonating nozzles cause jets to be structured with large discrete vortex rings that promote cavitation to depths several times greater than for conventional jets. The new nozzle designs are shown to be suitable for existing mechanical drill bits and may even affect hole cleaning in the absence of cavitation.

\section{INTROOUCTION}

It is generally accepted in the deep-hole drilling industry that bit hydraulics has a major effect on the rate of penetration. The actual drilling rate is always equal to or less than the rate which can be achieved if chips are removed as soon as they are created $[1,2]^{1}$. Chips are removed from the bottom of the hole by the drilling fluld. Since the total discharge through the bit is 1 imited by restrictions on the maximum (and mimimum) velocity up the annulus, the details of how this limited discharge is used to clean the hole bottom within the confined space available within the bit envelope is cruclal to the bit performance.

Most roller bits utilize the hydraulic horsepower avallable by ejecting the fluid at the maximum velocity possible from single orifices located in the sectors between the roller cones. In many designs these orifices are located at elevations above the hole botton which are comparabie to the cone diameter. The diffusion of these submerged jets is such that the maximum velocity reaching the hole bottom is greatly diminished. It is known that further increases in the jet velocity would improve the cleaning and thus the drilling rate, but, for the most part, the problems involved in working with higher pressure equipment have postponed development of this option. It has also been established $[3,4,5]$ that if the orifices are located closer to the hole bottom by using extension tubes, improved cleaning and thus increased drilling rates result, particularly when the drilling fluid contains fine solids (drilling mud) and there exists an overbalance in pressure across the hole bottom.

Illumbers in brackets designate References at end of paper.

Contributed by the Petroleum Division of the American Society of Mechanical Engineers for presentation at the Energy Technology Conference and Exhibition, New Orleans, Louistana, March 7-11, 1982.
Operating with an overbalance in pressure is the normal procedure to resist "blow outs". That is, the mud density is selected so as to produce a hydrostatic pressure at the hole bottom greater, than the formation pore pressure. Thus there is a flow of fluid into the formation and a resulting "filter cake" build-up across the hole bottom. Consequently the fluid passing over the hole bottom to remove chips must "lift" them against a very large pressure gradient - creating a hold-down force which is many times larger than the force caused by gravity. Fig. I illustrates qualitatively the dramatic decrease in drilling rate that results as the pressure difference across the hole botton is increased.

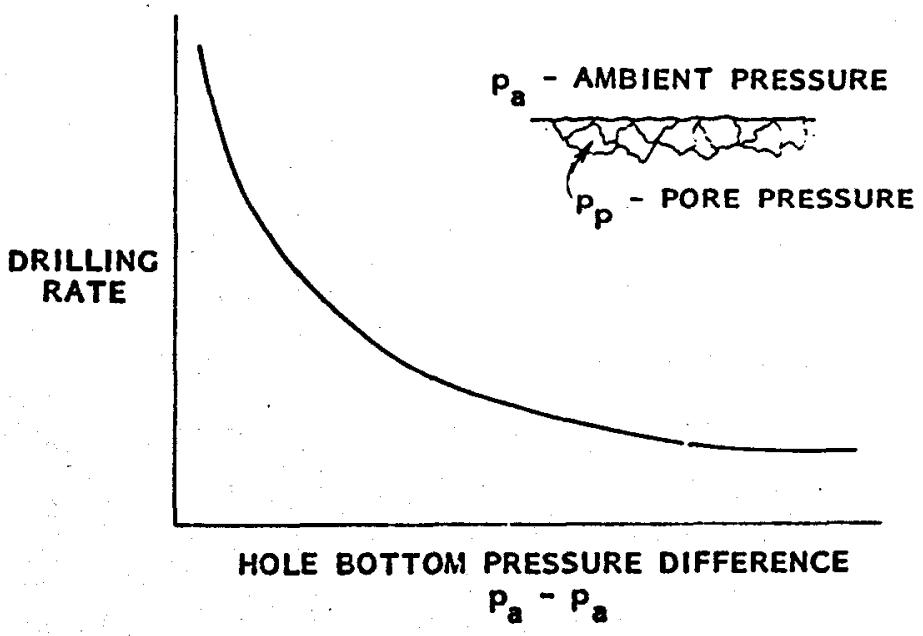

Fig. I Effect of overbalanced pressure on drilling rate

Clearly any "new" effect that could be utilized to improve the cleaning action of the jets used in mechanical drill bits might result in substantial increases in drilling rates. Our background in the mechanics of cavitation and cavitating jets initially led us to apply this "new" effect, using existing 
cavitating nozzle designs. Our recent and ongoing efforts, as described in this paper, involve studies to improve this cavitation effect by seeking passive means of causing the jet to structure Itself into periodic ring vortices. We now conjecture that this latter effect may improve cleaning of the hole bottom even when the operating conditions are such that cavitation cannot exist. This paper summarizes our work to date. Since our first attempts to incorporate cavitating jets into drill bits did not recognize the possibility of exciting the jets into a structured rino vortex formation, we.first summarize our past work on unstructured (or unexcited) cavitating jets and then describe the more recent work on structured jets both cavitating and noncavitating. It should be emphasized that this development of self-resonating jets is currently in progress and therefore some conclusions are, at this time, still preliminary.

\section{BACKGROUND-UNSTRUCTURED CAVITATING JETS}

\section{Cavitating Jet Principles}

The mechanics of cavitating submerged jets and the application of the intense erosion associated with cavitation to variety of cleaning and cutting applications has been studied at HYDRONAUTICS, Incorporated for over ten years. [6-10]. Cavitating and noncavitating jets are compared in Fig. 2 .

The important parameter defining cavitation is the cavitation number, $\sigma$ :

$$
\sigma=\frac{P_{a}-P_{v}}{H_{z} V^{2}}
$$

where: $P_{a}$ is the ambient or far field pressure $P_{v}$ is the vapor pressure of the liquid

$\rho$ is the liquid density

$V$ is a characteristic velocity, the jet mean velocity

In the case of high pressure submerged jets used in deep-hole drilling, $\mathrm{Pa}_{\mathrm{a}}$ is $\gg \mathrm{p}_{\mathrm{Y}}$ and for well-designed nozzles $\frac{1}{z} \rho V^{2}$ may be approximated as the pressure drop, $\Delta p$, across the nozzle. Thus:

$$
\sigma=\frac{p_{a}}{\Delta p} \text {. }
$$

The particular value at which cavitation is incipient is defined as:

$$
\sigma_{i}=\left(\frac{p_{a}}{\Delta p}\right) \text { at inception }
$$

\begin{tabular}{|c|c|}
\hline $\mathbf{a}$ & acceleration \\
\hline c & speed of sound in fluid \\
\hline$c_{m}$ & added mass coeffictent \\
\hline d & nozzle diameter (with subscript) \\
\hline D & diameter of pipe \\
\hline$f$ & frequency \\
\hline K & pressure coefficient \\
\hline$K_{n}$ & mode parameter \\
\hline$\ell, L$ & length \\
\hline$M$ & Mach number, $V / C$ \\
\hline$n$ & mode number \\
\hline$p$ & pressure \\
\hline$\Delta p$ & nozzle pressure drop \\
\hline $\mathbf{r}$ & radius \\
\hline$R_{e}$ & Reynolds number, $\mathrm{Vd} / \mathrm{v}$ \\
\hline $\mathbf{S}_{\mathbf{d}}$ & Strouhal number, $s_{d}=f d / V$ \\
\hline $\mathbf{t}$ & time \\
\hline$u^{\prime}$ & excited fluctuating velocity \\
\hline$v^{\prime}$ & excitation fluctuating velocity \\
\hline $\mathbf{v}$ & Jet velocity \\
\hline$w$ & pulse width \\
\hline$x$ & distance from orifice \\
\hline
\end{tabular}

\section{NOMENCLATURE}

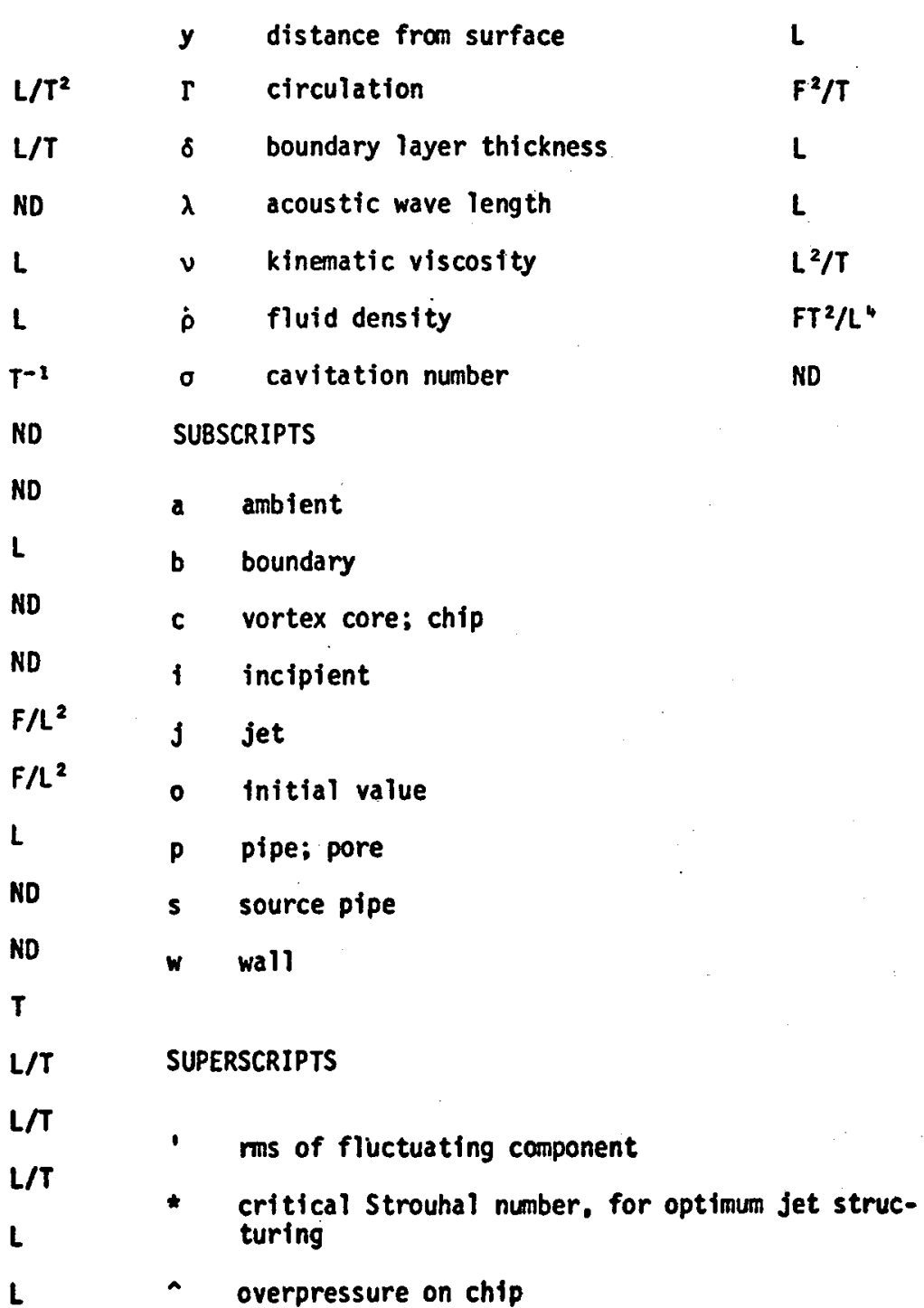




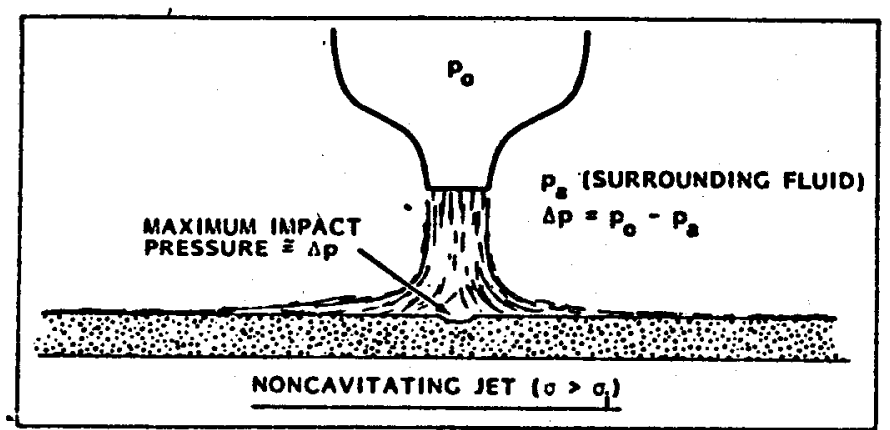

$0 \equiv \frac{P_{\mathbf{z}}}{\Delta p}$

$v_{i}=\left(\frac{P_{0}}{\Delta p}\right)$ INCIPIENT CAVITATION

$s_{i}=$ (SHAPE, JET DIAMETER, VELOCITY, VISCOSITY, FREQUENCY)

CAVITATION NUMBER.

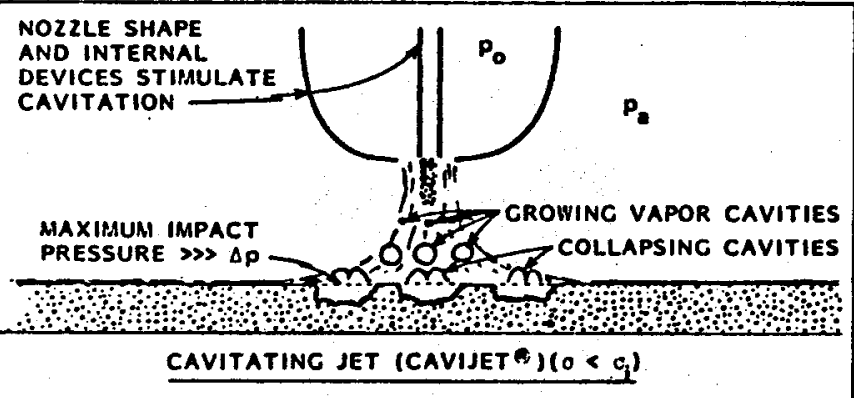

Fig. 2 Comparison of cavitating and noncavitating submerged jets.

Thus if the operating conditions for a submerged jet are such that $\sigma / \sigma_{j}>1$ then cavitation does not occur. When $\sigma / \sigma_{i}<1$ cavitation will occur and as $\sigma / \sigma_{i}$ continues to decrease below unity the amount of cavitation will increase. When a cavitating jet is impinged against a surface, so that the cavities formed by the jet are collapsed on that surface, very high presssures $\left(\gg \mathrm{k}_{\mathrm{s}} \mathrm{V}^{2}\right.$ ) are generated and the resulting cleaning or cutting action is substantially greater than when the jet is not cavitating. Thus as $\sigma / \sigma_{j}$ is reduced below unity the erosion intensity increases, usually reaching a maximum in the range $0<\sigma / \sigma_{1}<0.5$, and is always greater than the erosion intensity achieved for the noncavitating condition $\sigma / \sigma_{j}>1$.

As illustrated in Fig. 2, the local dynamic pressure reductions which cause cavitation in a submerged jet formed by a simple contracting nozzle are the result of the eddies created in the shear zone surrounding the jet. We have found that the magnitude of these pressure reductions depends on the boundary layer thickness at the nozzle exit. Nozzles we. now call "conventional" CAVIJET nozzles have been developed which minimize the boundary layer thickness and thus give maximum values of $\sigma_{j}$ for a given ratio of supply pipe diameter, $D$, to jet diameter, $d$. Addttional zones of pressure reduction may be created in the jet by swirling the flow so as to create a vortex down the center of the jet or by introducing bluntbased bodies within the center of the jet which cause cavitation in the eddies created in the resulting wake flow. We have found that cavitation caused to occur in the center of the jet is most damaging because these cavities can be made to collapse on the boundary (a surface beneath the impinging jet) within the stagnation pressure zone which is always greater than the ambient pressure. The ambient pressure more nearly represents the pressure which collapses the shear zone cavitles.

This paper discusses our work related to improving the erosivity of jets placed in mechanical bits which are used in drilling ofl, gas, or geothermal wells. The fluid pumped through the bit nozzles is predominantly drilling mud which is very erosive. Furthermore, the nozzles are subject to clogging if minimum acceptable clearances are not maintained. These facts weigh heavily against the use of central devices in most drill bit nozzles and thus such devices are not considered further in this paper.

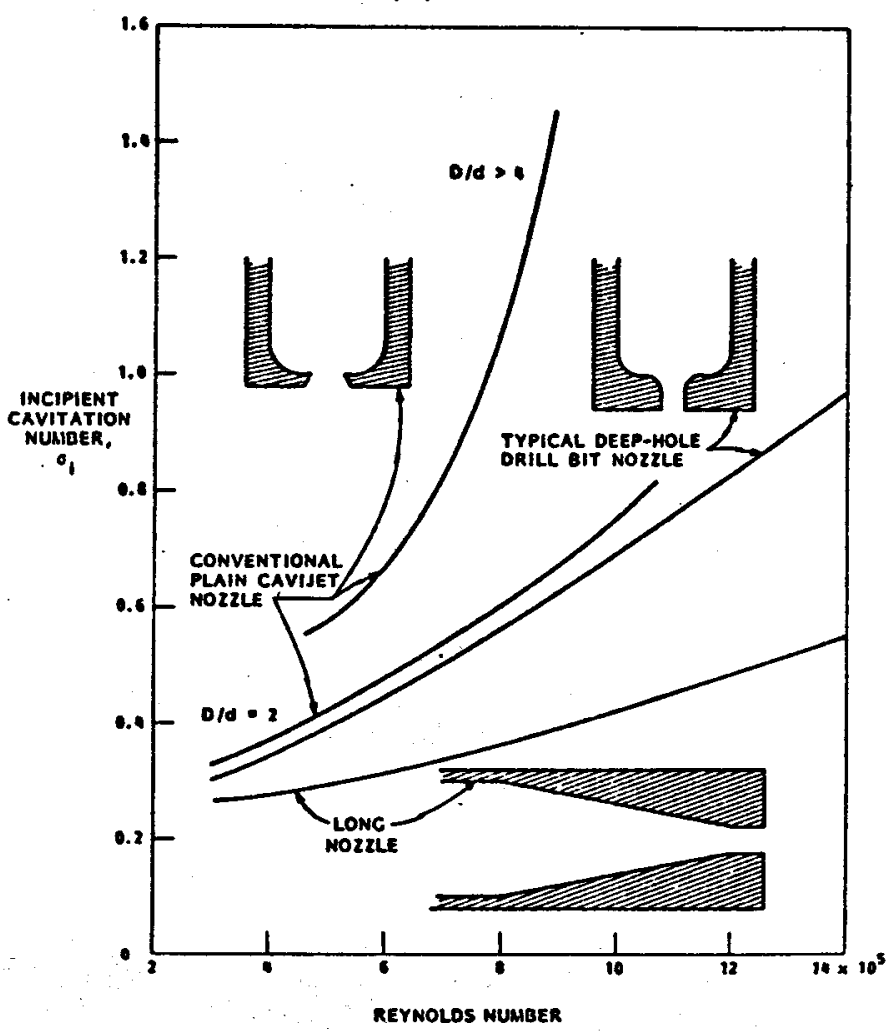

Fig. 3 Approximate Inclplent cavitation numbers for CAVIJET and other nozzle types (Acoustic detection)

Fig. 3 presents the approximate incipient cavitation number, $\sigma_{j}$, as influenced by the Reynolds number (based on nozzle diameter and jet velocity) for three types of "plain" nozzles (1.e., no central devices). Cavitation for such jets occurs in the shear zone around the jet periphery. These approximate curves represent data obtained from the literature [11] and from tests conducted in our laboratory. The values given are considered approximate because there is generally considerable scatter in data obtained in different facilities because of air content and nuclei effects, details of construction, and free stream turbulence. The important points to note in Fig. 3 are the significant differences in of that resuit for the three general classes of nozzles indicated and that the incipient cavitation.index for the conventional CAVIJET nozzle with $D / d=2$ is about the same as for a typical drill bit nozzle. It should also be noted that the higher values of of achievable with the conventional CAVIJET require $0 / d=4$ or greater.

Fig. 4a is a plot of the definition of $\sigma_{1}$ for various operating conditions. Since the amblent pressure in deep-hole drilling is hydrostatic, the ordinate 


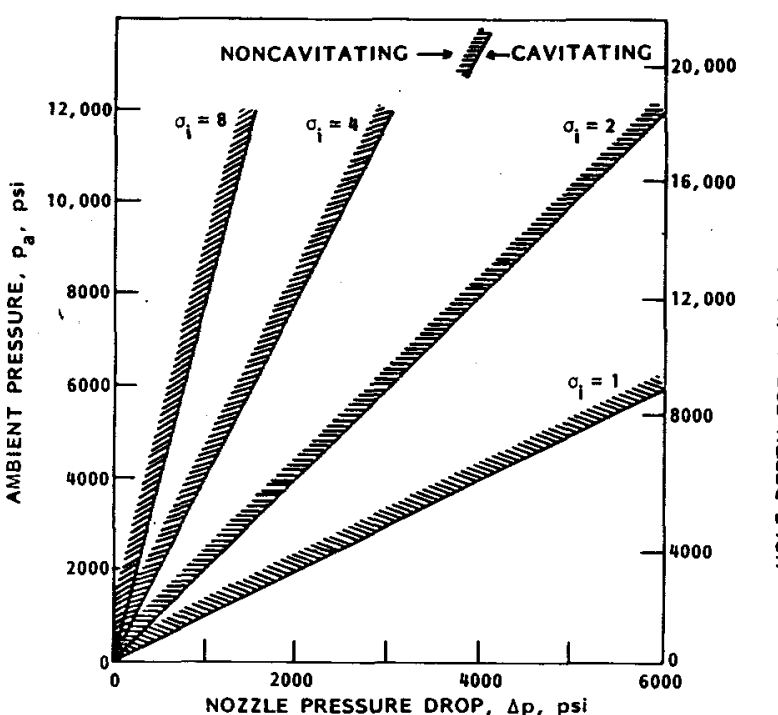

a. CAVITATING AND NONCAVITATING REGIONS

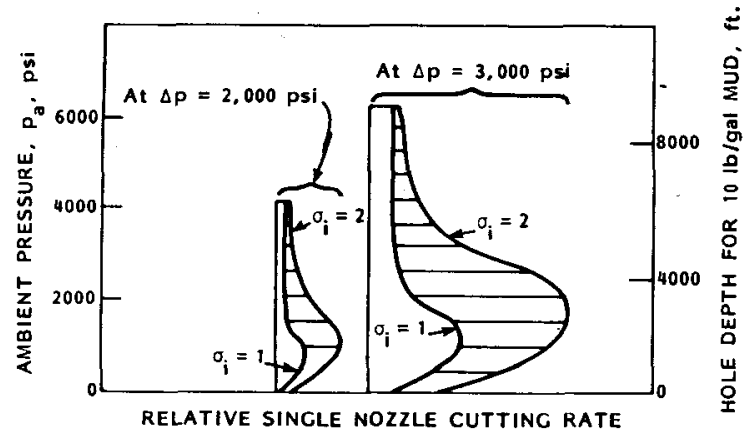

b. RELATIVE CUTTINC RATES

Fig. 4 The influence of $\sigma_{i}$ on the possibility of cavitation utilization for deep-hole drilling

may be related to hole depth for a given fluid density. In Fig. $4 a$ the right side ordinate is shown for a drilling mud density of $10 \mathrm{lb} / \mathrm{gal}\left(1.2 \mathrm{gm} / \mathrm{cm}^{3}\right)$. Referring to Fig. 3, for a Reynolds number of $10^{6}$ (typical for present drill bit operating pressures of 1,000 to $2,000 \mathrm{ps} j(6.9$ to $13.8 \mathrm{MPa})$ and the usual range of orifice sizes), the value of $\sigma_{j}$ for a $D / d \simeq$ 4 CAVIJET is approximately 1.8; for typical drill bit nozzles (or a $D / d \simeq 2$ CAVIJET) $\sigma_{j}$ is approximately 0.7 . Fig. 4a shows that cavitation will take place at an operating pressure drop of 2,000 psi (13.8 MPa) at depths less than about $2,500 \mathrm{ft}(760 \mathrm{~m})$ for conventional drill bit nozzles $\left(\sigma_{i}=0.7\right)$ and less than about $6,600 \mathrm{ft}(2,000 \mathrm{~m})$ for $D / d \simeq 4$ CAVIJET nozzles. Shown in $\mathrm{Fig}$. $4 \mathrm{~b}$ are relative nozzle cutting rates for $\sigma_{i}=1.0$ and 2.0 at $\Delta p=2,000$ and 3,000 psi $(13.8$ and $20.7 \mathrm{MPa})$. Most of the increased cutting (or cleaning) should occur for $\sigma / \sigma_{i}$ less than approximately 0.5 . Thus, the depths at which significant cavitation effects are to be expected would be (for $\Delta p=2,000 \mathrm{psi}(13.8 \mathrm{MPa})) 1,250 \mathrm{ft}(380 \mathrm{~m})$ for present drill bit nozzles and $3,300 \mathrm{ft}(1,000 \mathrm{~m})$ for a $D / d \simeq 4$ CAVIJET nozzle. Although the $D / d \simeq 4$ CAVIJET nozzle nearly triples the depth over which cavitation can have an effect, increasing the drilling rate in the first $3,300 \mathrm{ft}(1,000 \mathrm{~m})$ of a $25,000 \mathrm{ft}$ $(7,600 \mathrm{~m})$ deep hole will not have much of an effect on total drilling cost. On the other hand, if operating nozzle pressure drops were increased substantially, the range of depths over which cavitation will play a role would increase proportionately and the intensity of erosion (which has been found to vary approximately as $(\Delta p)^{3}$ ) would greatly increase. Furthemore, if the value of $\sigma_{i}$ could be increased to four or more, the depth over which cavitation contributed to improved drilling rate becomes significant even at current rig operating pressures.

\section{Drilling Tests}

In order to demonstrate that drilling rates can be increased when the cavitation effect is optimized we conducted laboratory tests on individual nozzles with drilling mud as a fluid and then replaced the nozzles of a 7-7/8 in. $(20 \mathrm{~cm})$ diameter SMITH A-1 two-cone bit with equivalent $D / d=4$ CAVIJET nozzles . This bit was tested under controlled conditions at the Drilling Research Laboratory. The results of these tests are reported in $[9,12]$. Fig. 5 shows this SMITH two-cone bit with CAVIJET nozzle installed. Fig. 6 shows the improved drilling rates in limestone which were observed for a nozzle pressure drop of $2,400 \mathrm{psi}(16.5 \mathrm{MPa})$. The trend in improved drilling rate is consistent with the foregoing discussion related to having a $\sigma_{i}$ approximately equal to 0.7 for the SMITH nozzles and 1.8 for the C.AVIJET nozzles.

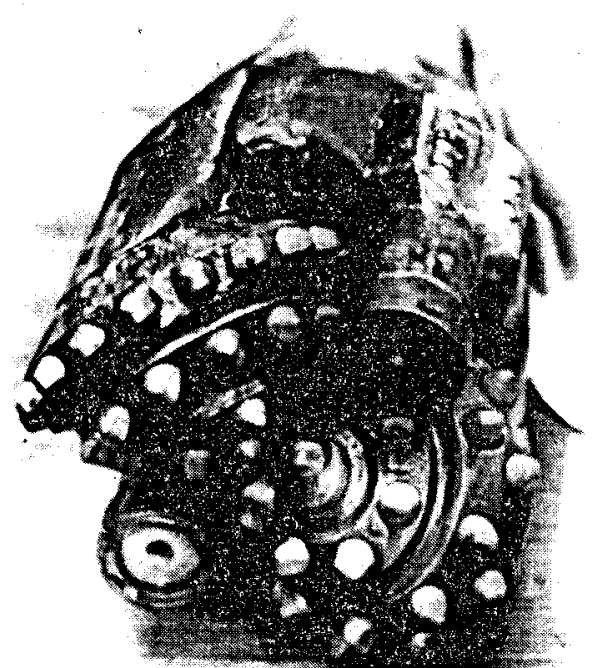

Fig. 5 Side view of two-roller bit: Showing extra extensions for two extended CAVIJET ${ }^{\left({ }^{3}\right.}$ nozzles

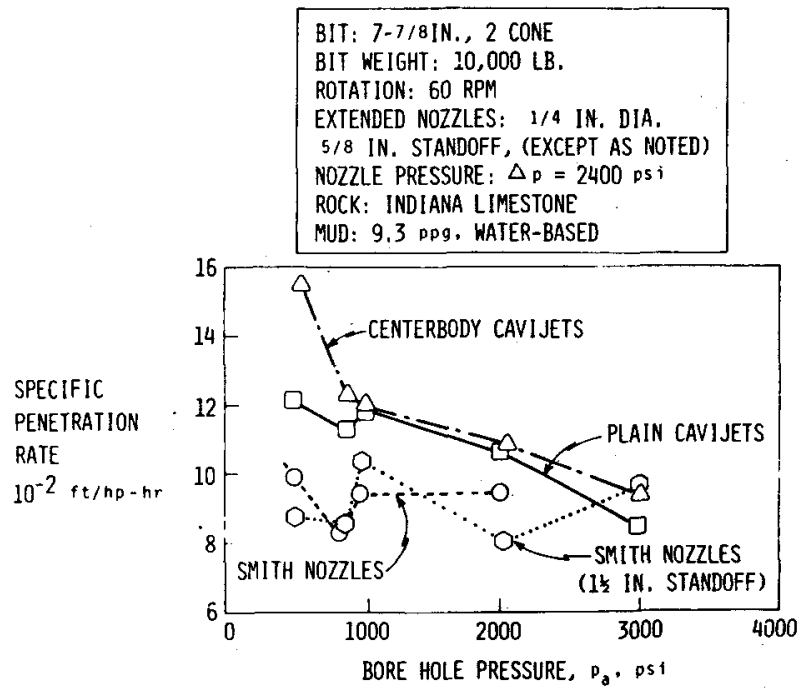

Fig. 6 Comparison of specific penetration rates 

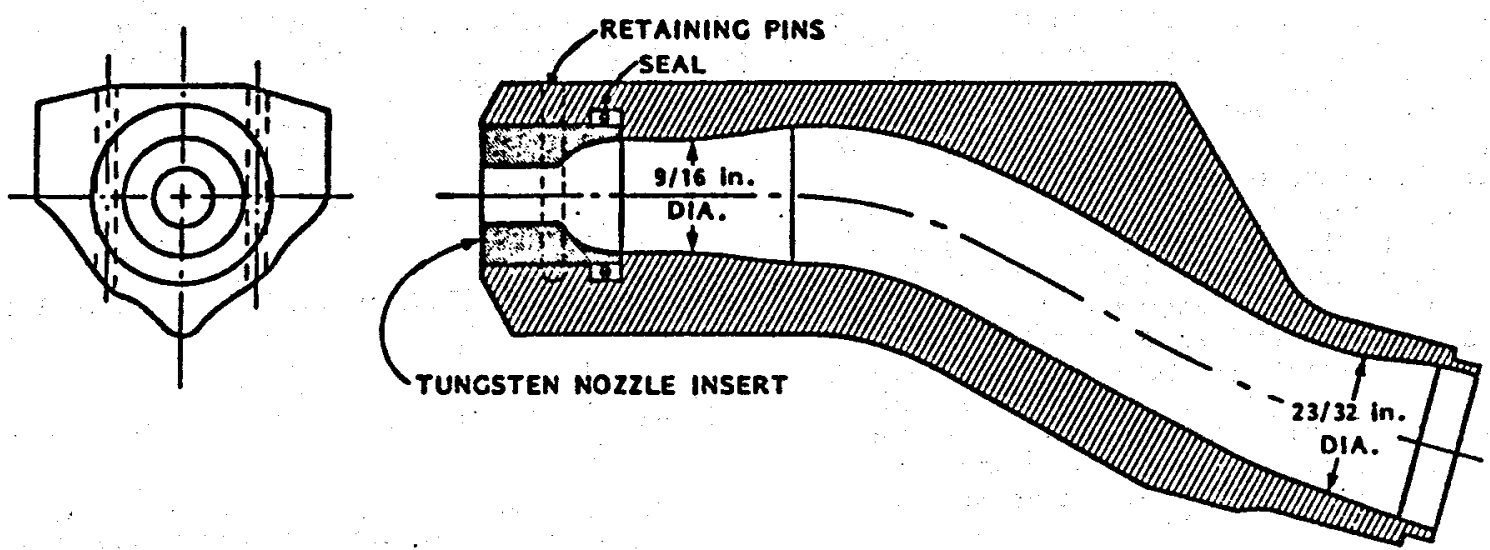

Fig. 7 Extended tube casting and insert for a typical 9-7/8 Inch, 3-cone roller bit

Subsequently nearly 20 SMITH A-1 bits ${ }^{2}$ have been tested in the field. Carbide nozzles and adapters required for these tests were provided by NL Hycalog and most of the tests were arranged (through NL) by the SMITH Tool Company with the Shell oil Company. of the tests conducted, about 50 percent were inconclusive; among the other half, direct comparisons of the $A-1$ bit with CAVIJET versus the $A-1$ bit with SMITH nozzles were scarce. In the direct comparisons, the CAVIJET bit drilled about 50 percent faster. In the indirect comparisons the CAVIJET bit was reported to drill 50 to 200 percent faster. In view of the relatively few tests conducted we conclude that the CAVIJET A-l bit performs at least as well as the DRL controlled laboratory tests indicate.

Since the CAVIJET nozzle is designed to have a high jet contraction ratio, the discharge coefficient of these nozzles is substantially less than conventional nozzles. Thus the equivalent CAVIJET nozzle is always larger in diameter. This is advantageous in drill bits since it reduces the possibility of clogging.

The SMITH A-1 two-cone roller bit was selected for tests with the $D / d=4$ CAVIJET nozzles because the inside diameter of its extended arms was large enough to install the $D / d=4$ nozzles. Field trials were difficult to obtain because this type of bit is used relatively infrequently. However, it served the purpose of lllustrating that drilling rates can be improved by the cavitation mechanism.

Fig. 7 shows the extended arm of a SMITH 9-7/8 in. $(25.1 \mathrm{~cm})$ three-cone roller bit. The three-cone bit is used in the majority of deep-hole drilling as opposed to the two-cone bit which is used only under special conditions. The inside diameter of this arm (D) cannot be significantly greater than $3 / 4$ in. ( $19 \mathrm{~mm}$ ). Typlcal nozzle orifice diameters (d) used with this bit are about $12 / 32$ in. $(9.5 \mathrm{~mm})$. Thus, the maximum $0 / d$ for nozzles installed in this bit is about two. Fig. 3 shows that CAVIJET shapes with $D / d=2$ have values of $\sigma_{j}$ that are not significantly different from conventional drill bit nozzles. Other types of mechanical bits such as diamond or Stratapax bits are also constructed so that it is generally impractical to use nozzles with values of $0 / d ₹ 2$. Thus if improved nozzles are to be found which.will have a significant impact throughout the drilling industry, they must have practical values of $0 / d<2$.

Although this important limitation in the supply pipe/nozzle diameter ratio was not the initial impetus

2 Manufactured by SMITH TOOI, Division of Smith International. Inc., Irvine, California, U.S.A. for our investigation of self-excited resonating nozzles it soon became a major motivating factor. He recognized that great increases in $\sigma_{i}$ might be achievable even for nozzles with $0 / d \leq 2$.

\section{STRUCTURED JETS - THEORY AND CONCEPTS}

Effects of Jet Structuring on Cavitation Inception

In [13] Crow and Champagne reported the remarkable change in the structure of an air jet when its driving pressure was excited periodically at certain frequencies by a loud-speaker so as to produce an oscillation in the exit velocity of only a few percent. Fig. 8 illustrates this change in structure. The frequency, $f$, required for essentially complete structuring of the jet into discrete vortices was defined by a Strouhal number, $S_{d}=f d / V$, of approximately 0.3 for the nozzle used (where $d$ and $v$ are the nozzle diameter and the jet velocity respectively). We recognized that the phenomenon described by Crow and Champagne should have an application in improving the performance of cavitating submerged jets if the excitation provided by the loud-speaker could be somehow passively provided in a feedback oscillator system. The results of our initial studies into the feasibility of such a scheme are reported in [14].

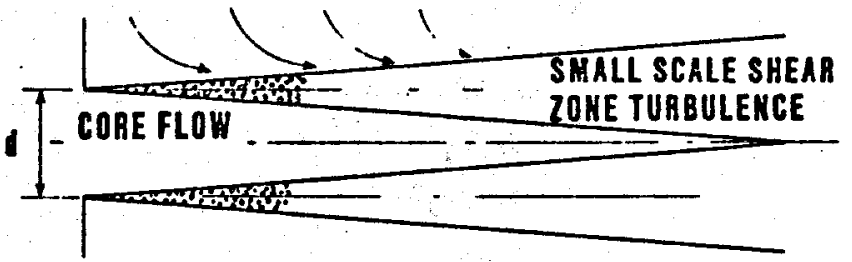

TRADITIONAL FLOW PATTERN

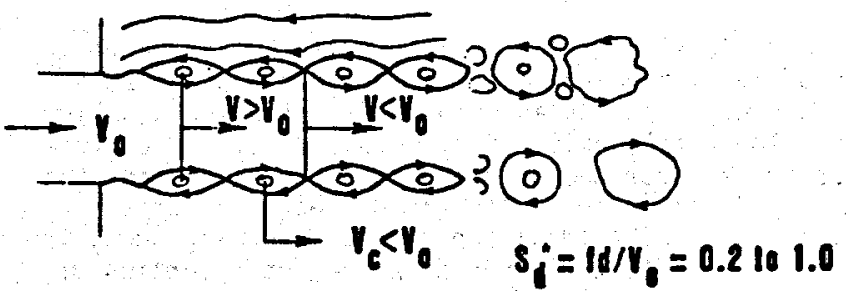

STRUCTURED FLOW PATTERN

Fig. 8 Submerged jet flow patterns 
There are two reasons why structured jets should be of interest in the context of improving drill bit performance. Both of these reasons may be revealed by estimating the expected differences in local pressures in the flow field and on the boundary (Y.e., the surface on which the jet is impinging) between an unstructured and a structured jet.

Fig. 9 shows the velocity distribution in a line vortex rotating in the direction shown having a forced (rotational) core radius denoted as $r_{c}$ and a velocity at $r_{c}$ equal to $V_{c}$. Such a Rankine vortex is a reasonable approximation of vortices which exist in real fluids having viscosity. For such a single line vortex, the value of the pressure drop from the ambient pressure. $\mathrm{Pa}_{\mathrm{a}}$, to the minimum pressure $\mathrm{P}_{\text {min }}$ (as shown in Fig. 9) which exists at the center of the core (and reaches the vapor pressure, $P_{i}$, at inception) is

$$
\frac{P_{a}-P_{\min }}{h_{2} V^{2}}=\frac{\Gamma^{2}}{2 \pi^{2} V^{2} r_{c}^{2}}=0_{1}, \text { (vortex ceriter) }
$$

where $\Gamma$ is the circulation around the vortex. That is,

$$
r=\phi_{v} \cdot d s=v \lambda
$$

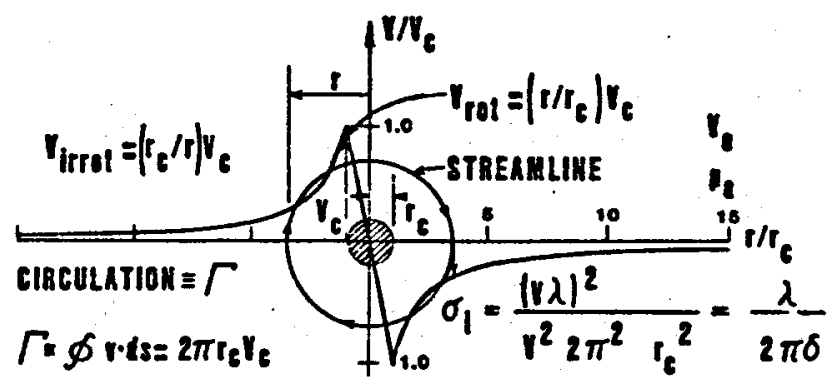

VELOCITY DISTRIBUTION

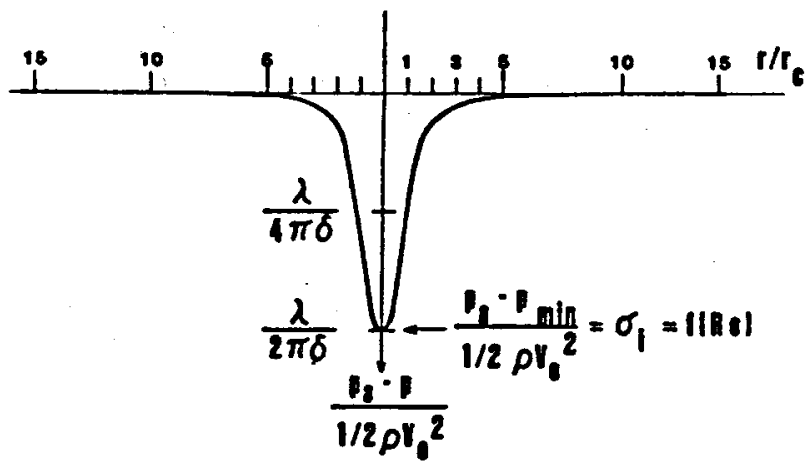

PRESSURE DISTRIBUTION

Fig. 9 Velocity and pressure distribution in a line "Rankine" vortex

Fig. 10 illustrates schematically how the core size of ideal ring vortices formed in the shear zone of a submerged jet is assumed to be established. Flow leaves the nozzle exit, of diameter, $d$, with a uniform velocity, $V$, over the nozzle exit plane except for the boundary layer region, which is of characteristic

thickness, 8 . The ideal shear zone, assuming no mixing with an outer fluid, is shown in the upper portion of the nozzle. In a real flow, exterior fluid is entrained and Rankine vortices form, with the rotational boundary fluid as the core. The lower portion shows how the distinct vortices, with a spacing denoted as $\lambda$, have a core made up of fiuid that has an area proportional to 28. If the core of these distinct vortices is assumed to be circular then

$$
r_{c}^{2} \propto \frac{\lambda \delta}{\pi}
$$

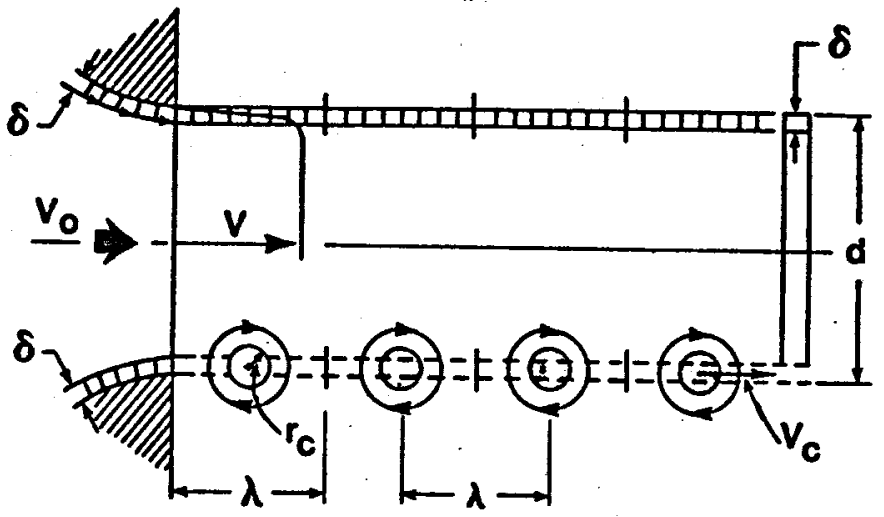

Fig. 10 Vortex roll-up in shear zone

The circulation of each vortex is obviously $\lambda V$. Thus, from equation (5)

$$
\sigma_{1, \text { (vortex center) }} \propto \frac{V^{2} \lambda^{2}}{V^{2} 2 \pi^{2} \frac{\lambda \delta}{\pi}}=\frac{\lambda}{2 \pi \delta} \text {. }
$$

Since $\sigma_{i}$ is desired to be as high as posstble in order to cause increased cavitation and erosion, it is preferable for a given nozzle, liquid and speed ( $\delta$ being fixed), to have $\lambda$ as large as possible. As shown in Fig. 8, for unexcited jets, the shear zone has many small vortices ( $\lambda$ is small and of order $\delta$ ) which roll up into a statistical distribution of sizes whereas, for an excited jet that becomes structured into discrete ring vortices, $\lambda$ is of the order of the jet diameter, d.

The preceding analysis is not exact because of the various simplifying assumptions made, (for example, the detailed pressure distribution in a ring vortex system is more complex) but the important result shown

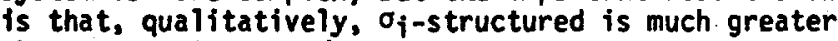

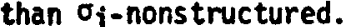

It is important to note the above-described increase in cavitation inception for a liquid jet excited at a preferred Strouhal number is entirely different from the increase that might obviously be assumed based on a quasi-steady state (i.e., nonstructured) analysis. That is:

$$
\left(\sigma_{i}\right)_{\text {nonstructured }}=\left(\sigma_{i}\right)_{\text {steady }}\left(1+\frac{v^{\prime}}{V}\right)^{2} \text {. }
$$

where $v^{\prime}$ is the magnitude of the excitation amplitude, and the maximum velocity $=v+v^{\prime}$. Very small amplitudes of excitation $(v \%=0.02)$ are required to achieve jet structuring. Thus according to equation (8a), negligible increases in $\sigma_{i}$ would occur. However. if the jet is structured, inception is given by:

$$
\left(\sigma_{i}\right)_{\text {structured }}=\left(\sigma_{i}\right)_{\text {steady }}\left(1+\frac{f u^{\prime}}{v}\right)^{2}
$$

where $u^{\prime}$ is the magnitude of the excited amplitude on the jet centerline a few diameters from the exit orifice, ( $u^{\prime} / V$ can be as large as 0.3 or more), and $f(>I)$ is a factor to account for the local maximum velocity in the structured vortices. Thus for structured jets, substantial increases in of are predicted by equation $(8 \mathrm{~b})$. 
The general effect described in the foregoing analysis is independent of the stand-off distance, $x$, $1 . e$. , the distance from the nozzle face to the fixed boundary to be eroded by a cavitating jet. In fact, this analysis neglected the boundary influence. We have determined that significant additional new cavitation effects should occur at relatively short stand-off distances, for example $x<6 d$. These effects are illustrated in Figs. Ila and 116 . The upper figure, $11 a$, shows an unexcited submerged llquid jet (with small scale random vortices) impinging on a solid boundary only a few diameters (d) away. The lower figure, 11b, 1llustrates a submerged liquid jet excited at a preferred strouhal number, with discrete vortices impinging on a solid boundary.

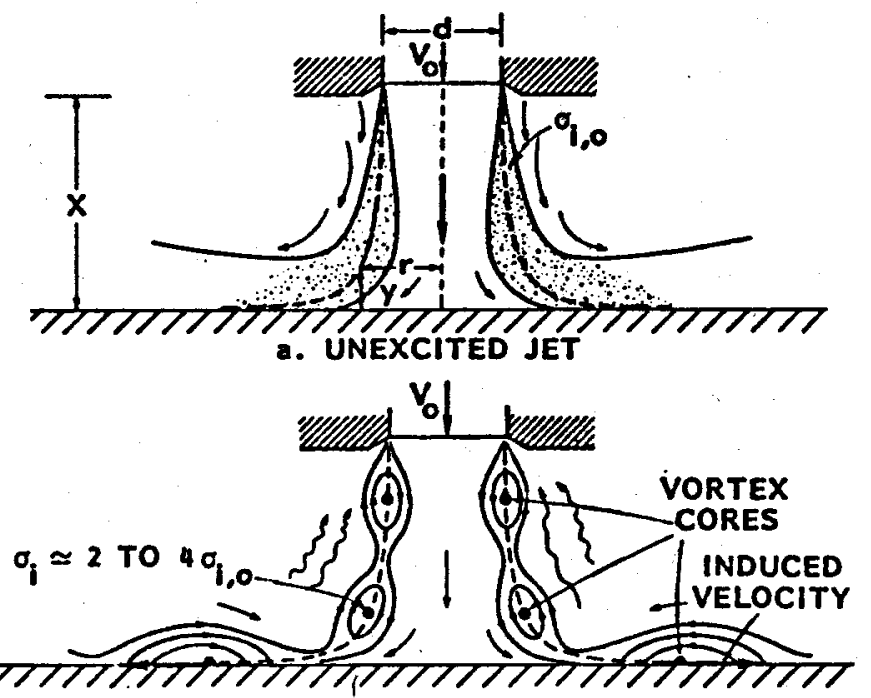

b. EXCITED JET

Fig. 11 Flow comparison of impinging submerged cavitating Jets

The dashed lines in Fig. ila and Ilb having coordinates $(r, y)$ represent the jet boundary that would exist if there were no mixing. It is assumed in Fig. IIb that the vortex centers lie on this path. For values of $\mathrm{r} / \mathrm{d} \geq 1$, this path can be obtained from the continuity equation (assuming the flow in this outer region is entirely radial). The approximate equation for this path is:

$$
\frac{y}{d}=\frac{1}{8} \frac{d}{r} \text { or } \frac{r}{d}=\frac{1}{8} \frac{d}{y}
$$

Thus, as the vortex rings approach the boundary ( $d / y$ increases and thus $r / d$ increases), the ring size increases. It is fundamental in hydrodynamics that such "stretching" of a vortex will result in a decrease in core size. In fact, if it is assumed that the core fluid in a ring of radius, $r$, redistributed to fill the same volume when the ring stretches to a new radius, $r_{2}$, the ratio of core sizes will be given by the following equation:

$$
\left(\frac{r_{c_{1}}}{r_{c_{1}}}\right)^{2}=\frac{r_{1}}{r_{2}}
$$

Thus, from equation (4):

$$
\frac{\left(\sigma_{1}\right)_{2}}{\left(\sigma_{i}\right)_{1}}=\frac{r_{2}}{r_{1}}
$$

Assuming that $\left(\sigma_{i}\right)$, , represents the value of $\sigma_{1}$ In a ring near the nozzle exit and thus away from the boundary, with $r_{1}=d / 2$, the value of $\left(\sigma_{i}\right) 2$ for a ring closer to the boundary, as given by equation (9) becomes:

$$
\begin{gathered}
\frac{\left(\sigma_{1}\right)_{2}}{\left(\sigma_{f}\right)_{1}}=\frac{1}{4} \frac{d}{y}, \\
\text { For } \frac{r}{d} \geq 1 \text {, thus } \frac{d}{v} \geq 8 \quad \ldots
\end{gathered}
$$

Thus, in the absence of viscous effects (core size growth due to viscosity and circulation decrease caused. by wall friction), cavitation should first occur in the vortices as they spread over the boundary rather than at their birth near the nozzle.: Experiments have shown that these effects tend to cause the actual core minimum pressure to occur somewhere between the exit orifice and $r / d=2$.

Possibly a more important influence of a boundary on the cavitation characteristics of an excited jet with discrete vortices is the reduction in pressure on the boundary that should result as a vortex spreads radially over it. This effect is also shown in Fig. 11b. In the absence of viscosity, the velocity field near the vortex of strength, $\Gamma$, in Fig. 11b varies inversely with distance from the vortex. The actual induced velocity at the boundary may be approximately determined by placing an image of the vortex within the boundary and is, for a vortex circulation of $V \lambda$ :

$$
V_{\text {induced }}=\frac{8 \cdot V \lambda}{\pi d} \cdot \frac{r}{d} .
$$

Thus the total instantaneous velocity, $v_{t}$, on the wall beneath a vortex as it sweeps over the boundary is approximately

$$
v_{t}=v_{w}-v_{c}+\frac{1}{\pi} \frac{v \lambda}{d} \frac{d}{y}
$$

where $V_{W}$ is the velocity near the wall in the absence of ring vortices, and $V_{C}$ is the convection speed of the vortices. The pressure at this point, from Bernoulli's equation, is given by:

$$
\frac{P_{a}-P_{\min }}{j \rho V^{2}}=\sigma_{i, \text { boundary }}=\left(1+\frac{1}{\pi} \frac{\lambda}{d} \frac{d}{y}\right)^{2}-1 \text {. }
$$

Substitution of equation (9) into equation (14) results in

$$
\sigma_{i, \text { boundary }} \approx\left(1+\frac{8}{\pi} \frac{\lambda}{d} \frac{r}{d}\right)^{2}-1 \text { for } \frac{r}{d}>1
$$

Equation (15) reveals that very high values of $a_{i}$, boundary will occur even for $r / d=1$; that is $\sigma_{1}$, boundary $=12($ for $\lambda / d=1)$.

As will be discussed below, the value given in equation (15) is also the negative of the minimum pressure coefficient, $K$, on the boundary. where

$$
K=\frac{P_{b}-P_{a}}{1 \rho V^{2}}=-\sigma, \text { boundary }
$$

This low pressure induced on the boundary will be significant in cleaning the bottom of deep holes drilled with mechanical bits which incorporate jets structured into discrete vortices.

Effects of jet Structuring on Bottom Hole Cleaning Equation (15) has been used to generate the approximate instantaneous pressure distribution on the boundary shown in Fig. 12. The pressure $\mathrm{pa}_{\mathrm{a}}$ and $\mathrm{Pb}_{\mathrm{b}}$ are the ambient pressure and the boundary pressure, respectively. The cross hatched rectangles represent constant value pulses of width, $w$, that approximate 
the actual pressure distribution. Although it is recognized that the vortex spacing will aiter as the rings approach and spread over the boundary, the assumption that this spacing is constant is not crucial to the general magnitude of the minimum pressures that will occur on the boundary; however, the relative locations may be different. Viscous effects will aiso modify the results shown in Fig. 12. Obviously friction and vortex break-down should have a large influence, particularly when $r / d ; 1$. However, as long as the vortices do not break down unusually high negative instantaneous pressures must occur on the boundary an order of magnitude greater than the nozzle pressure drop. Compare this with the turbulent fluctuations of an unstructured jet which are generally about an order of magnitude less than the nozzle pressure drop. If such large pressure fluctuations do result when a structured jet impinges on a boundary, they may be large enough to lift small chips against the pressure gradient forces at the hole bottom as discussed in the Introduction.
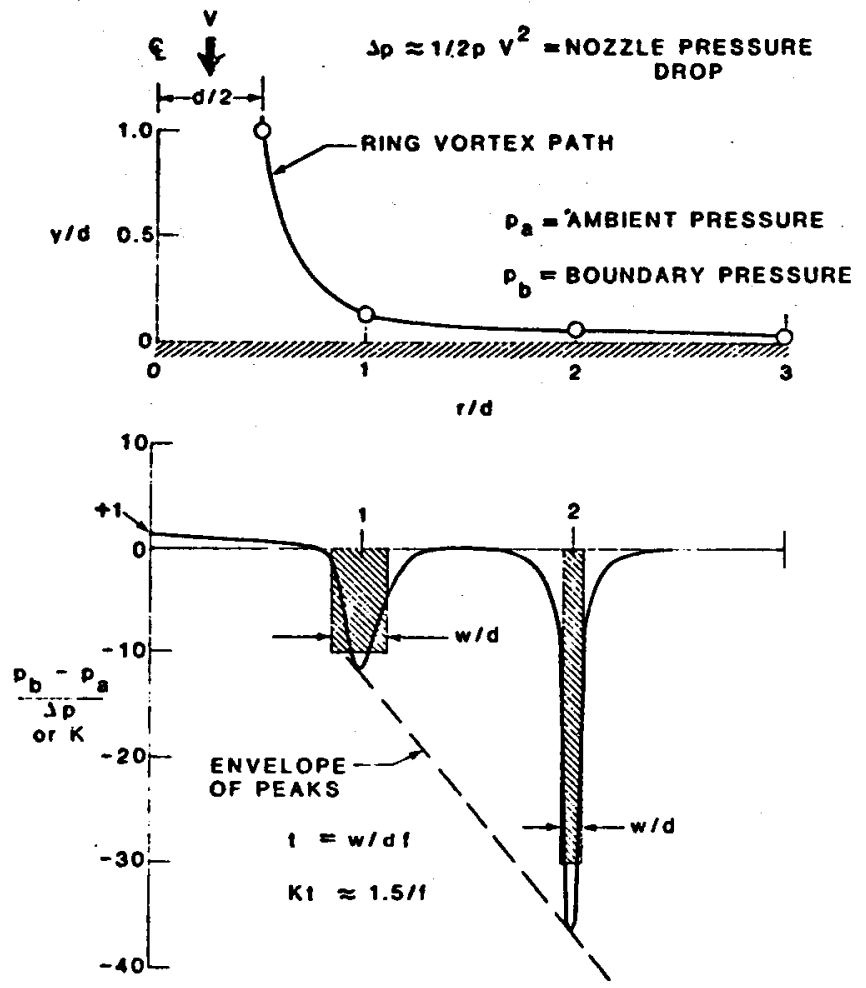

Fig. 12 Instanitaneous boundary pressures for structured.jet

A schematic drawing of the various pressures acting on a chip is shown in Fig. 13. Preliminary analyses (detailed in Appendix A) have shown that ample chip ifting fressures should be generated by the mechanism indicated in Fig. 12.

The ORGAN-PIPE CAVIJET Concept for Achieving A Passively structured Jet

In [14] several methods of passively achieving jet pulsation were shown to be feasible. Our recent work has concentrated on the ORGAN-PIPE CAVIJET concept shown in Fig. 14 because of its simplicity and because it shows the most promise of being adaptable to existing mechanical drill bits with only minor changes to the - hydraulic passages in the bits.

The principles involved in the ORGAN-PIPE CAVIJET concept are common to any passive resonating system. A jet forming nozzle is fed from a supply system that has a natural acoustic frequency equal to the critical frequency required by the nozzle for jet structuring. The jet forming nozzle must be shaped in such a manner as to feed back to the supply system pressure oscillations associated with the formation of ring vortices in the structured jet. These fed back oscillations cause resonance to occur in the supply chamber wilich in turn further excites the jet until the damping in the system causes equilibrium to be reached.

In spite of the simplicity of the ORGAN-PIPE CAVIJET concept, the detailed design of an optimum system has been found to be quite complex and our studies are not yet complete enough to present such an optimum example in this paper. However, the general approach for developing this design is as follows.

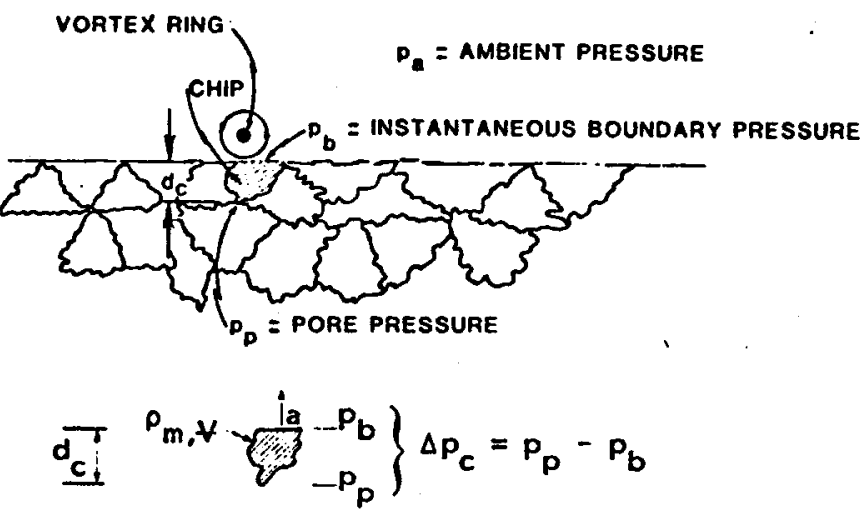

Fig. 13 Chip hold down

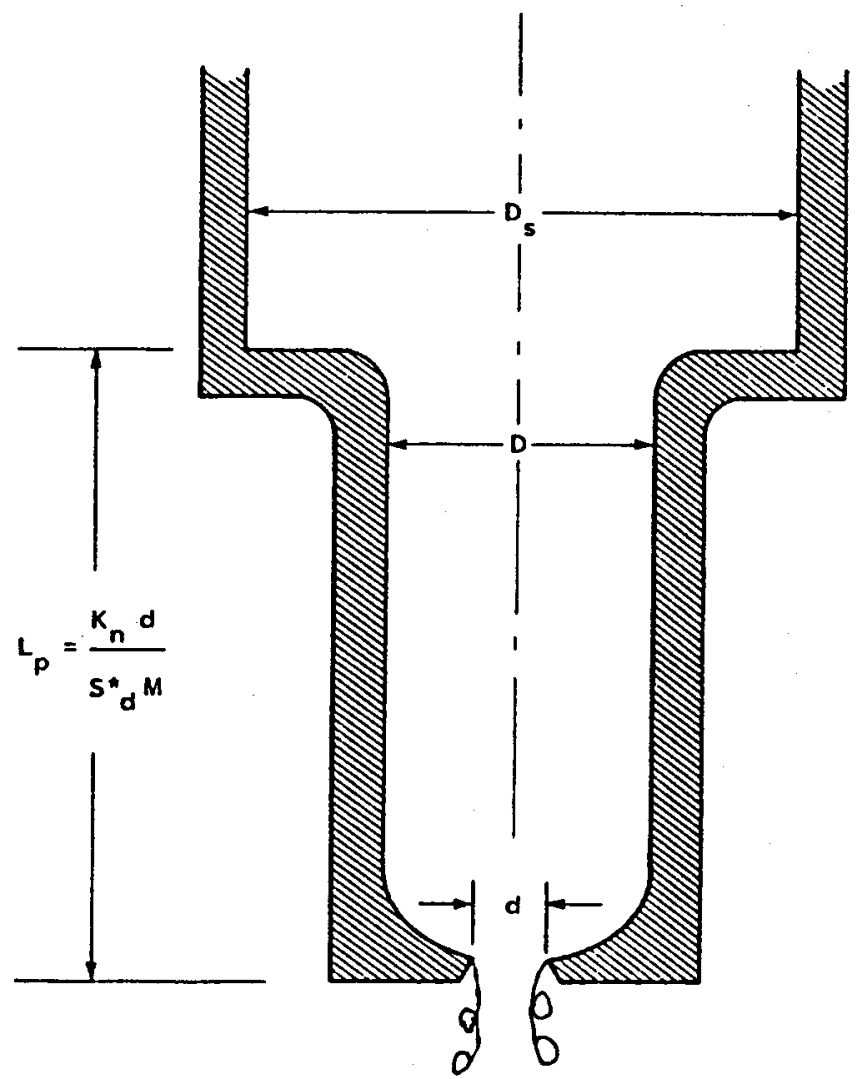

Fig. 14 Organ-pipe CAVIJET ${ }^{\oplus}$ nozzle concept 
The ORGAN-PIPE CAVIJET concept shown in FIg. 14 should achieve peak acoustic resonance when a standing wave forms in the "organ-pipe" section (length: $L_{p}$. diameter: 0$)$. This section is created by the upstream contraction; $\left(D_{s} / D\right)^{2}$ and the nozzle contraction $(0 / d)^{2}$. Peak resonance will occur when the frequency of the organ-pipe wave is near the critical jet structuring frequency, as given by the nozzle strouhal number, $S_{d}$, but the exact resonance is dependent on the contractions at each end of the organ-pipe. For instance, if both $(0, / O)^{2}$ and $(0 / d)^{2}$ are large, then the first mode resonance in the pipe will occur when the sound wave length in the fluid is approximately four times $L_{p}$. Acoustic analyses and experimentation have led to the following approximation, useful for estimating the length of the organ-pipe:

$$
\frac{l_{p}}{d} \simeq \frac{K_{n}}{M S_{d}}
$$

where the "mode parameter", $K_{n}$ is given by:

$$
\begin{array}{r}
K_{n}=F\left\{n,\left(\frac{D_{s}}{D}\right)^{2},\left(\frac{D}{d}\right)^{2}\right\} \\
=\frac{2 n-1}{4} \text { for }\left(\frac{D_{s}}{D}\right)^{2} \text { and }\left(\frac{D}{d}\right)^{2} \gg 1 \\
=\frac{n}{2} \text { for }\left(\frac{D_{s}}{\theta}\right)^{2} \gg 1, \text { but }\left(\frac{D}{d}\right)^{2}=1
\end{array}
$$

In these expressfons:

$n=$ mode number of the organ-pipe,

$S_{d} \star=$ critical Strouhal number, $f d / V=0.3$ to 0.8

$M=$ Mach number, $V / c$.

Other examples of ORGAN-PIPE CAVIJET concepts are shown in Fig. 15, where single diameter, "two-step", and "three-step" diameter versions are indicated. The reason. such multiple step supply pipes are of interest is related to our preliminary findings that the jet forming nozzle characteristics (feed back gain and critical strouhal number) are influenced by the final area contraction ratio. Furthermore, when a particular mode is sought, it may be necessary to "force" the mode by locating a step at the desired antinode location.

Including such steps also provides a means of minimizing the hydraulic pressure loss in the tube consistent with structural considerations.

Let us assume that the ORGAN-PIPE CAVIJET concept is to be applied to an existing extended nozzle threecone bit which is to be operated over a prescribed range of pressure drops. The overall length of the tube is fixed. Several nozzle sizes are required in order to maintain discharge over the range of pressure drops. As will be shown in the following section, we have found the tuned "width" (i.e. the width or range of Mach numbers in a resonance piot such as Fig. 2i) for strong oscillations of good ORGAN-PIPE designs is about \pm 15 percent of the design Mach number or about \pm 30 percent of the design pressure drop across the nozzle (for a given fluid density). This resonant peak width provides an adequate design range for most practical applications. For example, if the nozzle is designed for peak performance at a pressure drop across the nozzle of $1,500 \mathrm{psi}(10.3 \mathrm{MPa})$. structuring of the jet, and hence resonance. may be expected over a range of $i, 000 \mathrm{psi}<\Delta p<2,000 \mathrm{ps} i,(6.9 \mathrm{MPa}<\Delta p<13.8 \mathrm{MPa})$. Since the tube length is given and the Mach number is prescribed in the design. equation (17) shows that a nozzle configuration must be found for each desired orifice diameter which has a critical strouhal number.
$S_{d}$, that satisfies equation (17) with $K_{n}=n / 2$. Host of our current research has been directed toward finding nozzles which satisfy this criterion and in addition optimize the overail performance of the system. We have not progressed sufficiently to present a complete discussion on how the critical strouhal number is Influenced by nozzle geonetry, viscosity, rheology and cavitation number. However, we have conducted a number of experiments in air and water which are contributing to an improved understanding and which have demonstrated that practical solutions do exist. The following section describes some of this work.
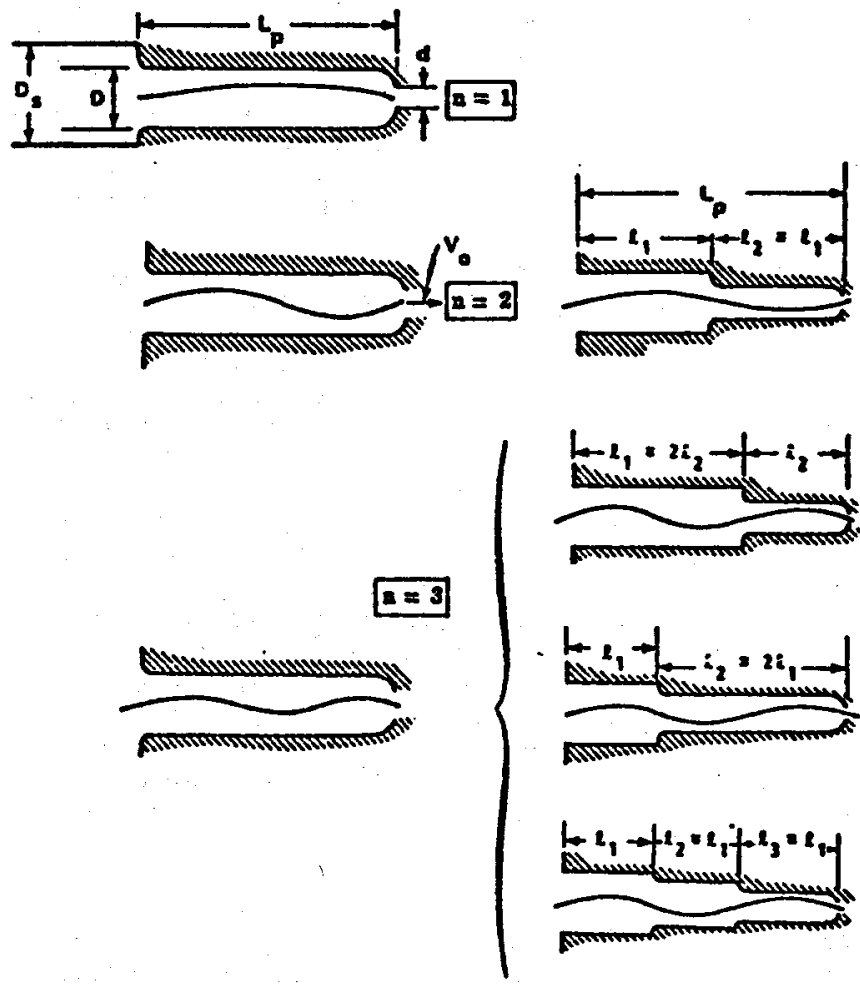

Fig. 15 Multiple stepped organ-pipe CAVIJET concepts

\section{STRUCTURED JET EXPERIMENTS}

\section{Experimental Facllities}

Two facilities were developed to study the characteristics of self-resonating jets. An air facillty allows examinations of jet performance with the capability of rapid and economical changes in a design iteration that are not so readily made in the highpressure water jet facility. Although the existing air facility has been operated at Reynolds numbers which are too low to allow complete scaling to the water results, valuable insights which have guided and minimized the more tedious tests in water were gained by studies of resonating air jets.

Air facility. A schematic of the components contained in this facility are shown in Fig. 16. The rectangular plenum supplied air to the nozzle being tested. Pressure in the plenum was controlled by a needle valve in the 30 pst $(0.2 \mathrm{MPa})$ supply itne from the air compressor, and this pressure was monitored by a $U$-tube manometer. Both free jet and impinging jet tests were run, with a movable target plate placed normal to the jet axis at various locations. Perturbations in the jet axial velocity were surveyed with a 25in diameter hot film sensor. This probe was mounted 


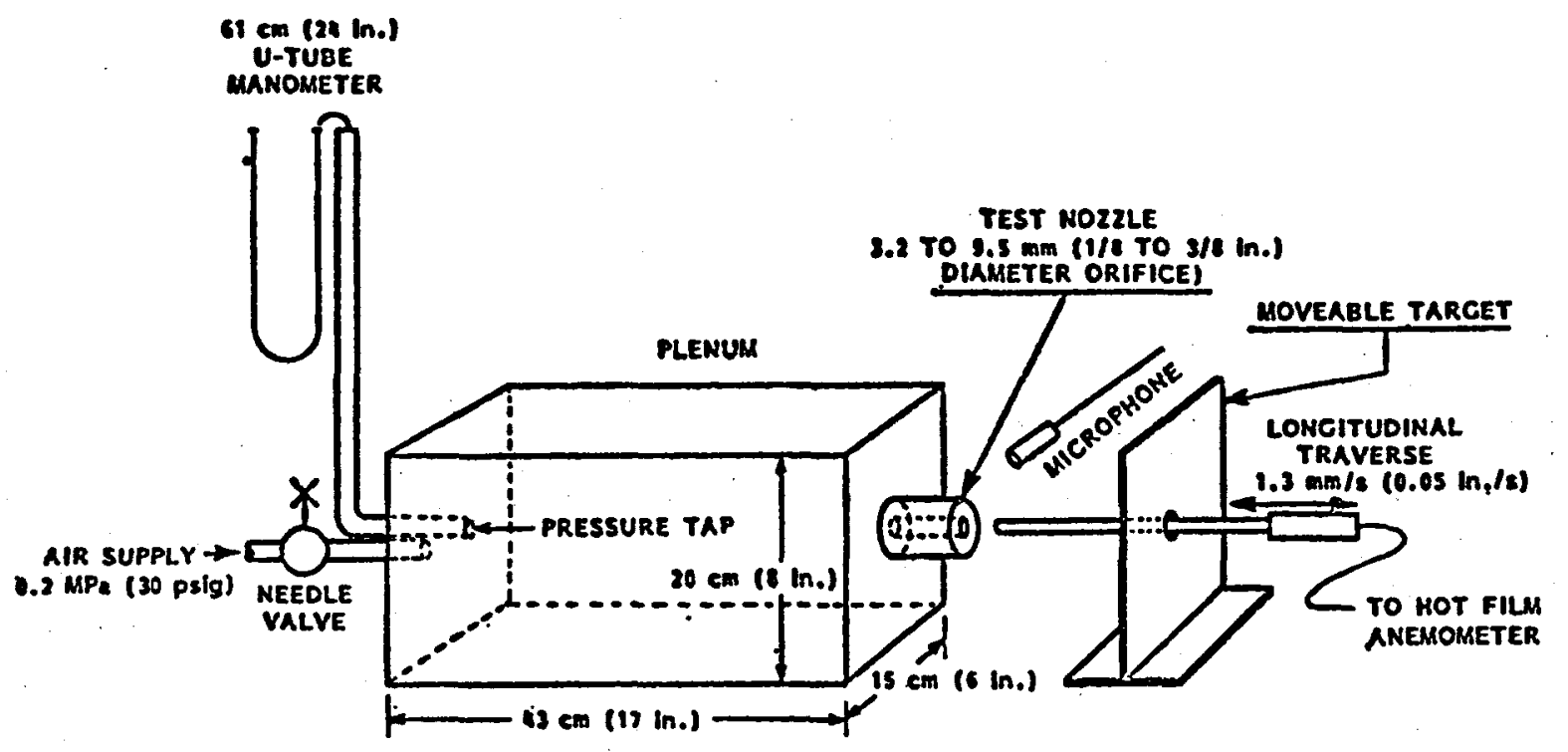

Fig. 16 Apparatus for nozzlo tests with alr

on motor driven rig, allowing continuous survey long either the axial or radial directions. A nearby microphone monitored acoustic signatures from the nozzle

The electronic signal from the hot film sensor was conveyed to a Thermo-Systems Model 1050 hot-film anemometer bridge. The output from this bridge was then fed directly to an RMS voltmeter, a spectrum analyzer

(Unigon Model 256), and an $X-Y$ plotter. By also sending the RMS voltmeter output to the plotter, side-by-side comparisons could be graphed of the mean, $V$, and fluctuating, $u^{\prime}$. velocities. Output from the spectrum analyzer was viewed on an oscilloscope, to allow identification of the resonant frequency peaks in the fluctuating velocity. The microphone output was also fed to an RMS voltmeter and to the spectrum analyzer. thus providing a monitor of the frequency and intensity of sound pressure levels. These components were also used to process the signals from the pressure probes used in the water tests.

High Pressure Cell (HPC). The test chamber used to observe the behavior of submerged cavitating jets is shown in Fig. 17. This cell can contain an internal pressure of up to 3,000 psi $(20.7 \mathrm{MPa})$. This cell ambient pressureis controlled by a "choke". a floating double-ended piston valve, which is balance by nitrogen gas. This floating action allows for the escape of particles during rock cutting trials. Rock specimens, typically cubes 6 in. $(15.2 \mathrm{~cm})$ on a side, can be rotated beneath the jet in the HPC, at rates up to $66 \mathrm{rpm}$ by means of the variable speed drive.

- The high pressure fluid for this cell is usually provided by either a three or a five-plunger pump; if a test requires higher flows then both pumps are run in parallet. The triplex pump, which is also rated for drilling mud, will deliver $80 \mathrm{gpm}(303 \mathrm{l} / \mathrm{m})$, at 2,000 psi $(13.8 \mathrm{MPa})$. The special quintuplex pump, with changeable plungers and head, can be run over range; highest flow: $90 \mathrm{gpm}(341 \mathrm{l} / \mathrm{m})$ at $2,500 \mathrm{ps} /(17.2 \mathrm{mPd})$. or highest pressure: $10,000 \mathrm{psi}(68.9 \mathrm{MPa})$ with a 20 gpm $(76 \mathrm{l} / \mathrm{m})$ flow capacity.

For flow visualization and photography, the HPC has been fitted with three circular viewing ports. each $1.5 \mathrm{in.}(3.8 \mathrm{~cm})$ in diameter. A typical photograph of a self-resonating cavitating jet taken in this ccll is shown in Fig. 26. This photograph was taken by darkening the room, opening the camera shutter, and releasing a single flash from a stroboscopic light source. When observing the jets by eye, the strobe is operated at a . suitable submultiple of the jet resonance frequency so as to reasonable "stop" the motion of the vortices. In this manner, jet structure can easily be monitored whlle operating parameters are varfed.
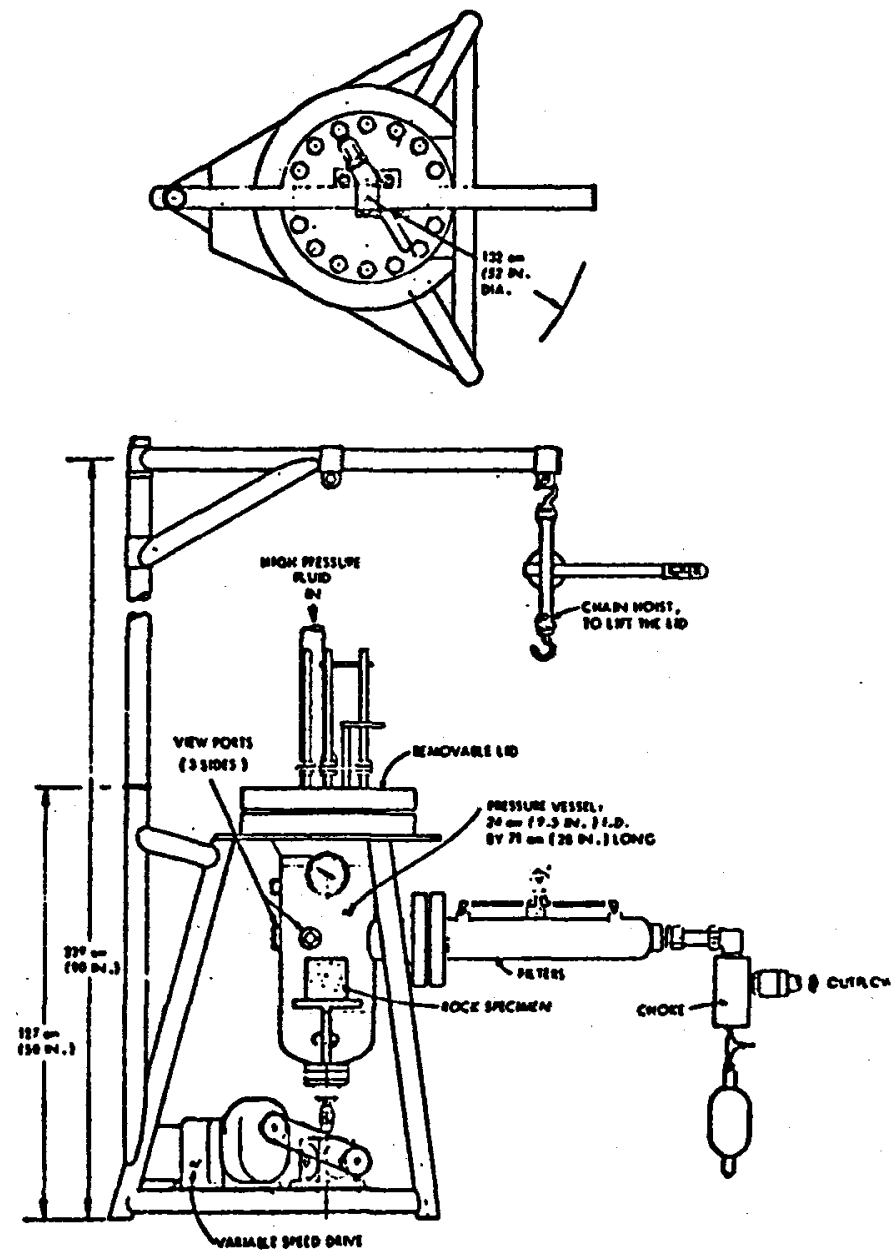

Fig. 17 High pressure cell (HPC) 
Two piezoelectric pressure transducers (PCB Piezotronics Model No. 101A04; 0.25 in. $(6.4 \mathrm{~mm})$ dia.) are mounted in the HPC. One is placed in the inlet supply pipe feeding the nozzle; the other is in the wall of the main pressure vessel. As noted above, the same instrumentation used for the air tests serves to process the signals from these pressure transducers.

\section{Jet Structuring Observations}

General Test Method. Well over a hundred different organ-pipe configurations have been investigated, both in air and in water. In these studies the supply pipes have been similar to those shown in Fig. 15. Most have been straight although some have utilized the actual curved extended tubes from SMITH roller bits. Some tests were conducted, in air, with a complete $12 \frac{1}{4}$ in. $(31.1 \mathrm{~cm})$ diameter SMITH F-3 three-cone bit as shown in Fig. 18 and runs were also made in water using a onethird sector of such a bit.

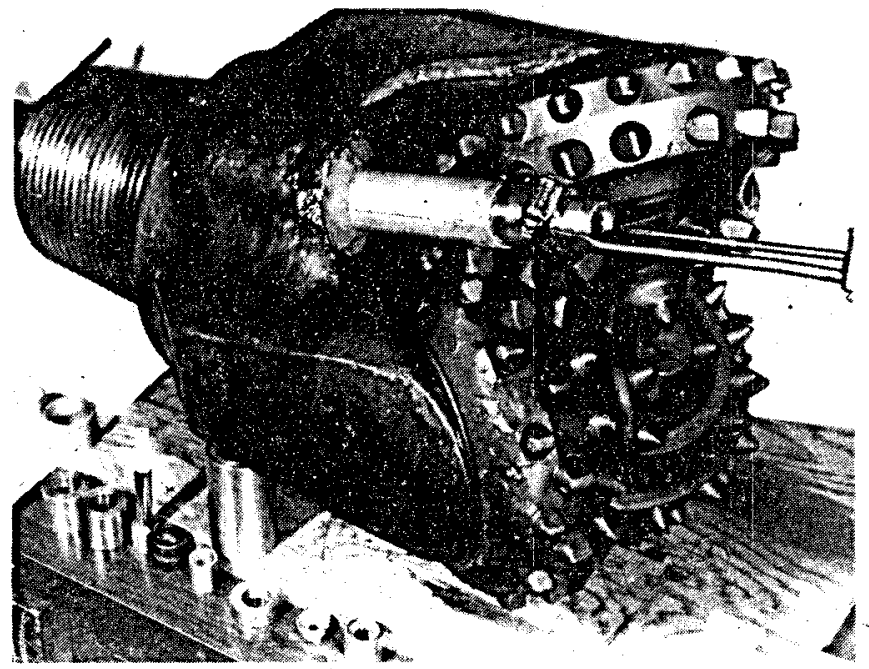

Fig. 18 Three cone bit test in air

In all of these tests the procedure involved changing the pressure drop across the nozzle so as to vary the jet velocity (for a fixed ambient pressure when testing in water) and observing the output from the anemometer or pressure transducer. It is generally very easy to observe when the jet becomes structured. If the frequency is in the audible range a pure tone is heard. The oscilloscope output from the anemometer or pressure transducer becomes periodic and grows in amplitude as a particular mode becomes excited. The magnitude of the fluctuating signal is recorded along with its frequency.

Natural Frequency. In most of the tests conducted. the observed resonant frequency was wi thin about 10 percent of the frequency which corresponds to multiples of a half wave length. Such a result is expected because the nominal supply pipe to orifice diameter in all of the tests conducted has been two or less. We have applied more sophisticated analyses [15 and 16] to determine the natural frequency for the actual area distributions tested and the agreement with measured frequencies was excellent: However, for preliminary design purposes equation (17), which is based on natural frequencies corresponding to standing waves in the tube of half wave length multiples, is adequate.

Fig. 19 compares equation (17) with the results obtained in air, using straight tubes with diameters of 0.6 to 1.2 in. and a nozzle having a critical
Strouhal number of approximately 0.45. Some tubes included steps located at antinodes. The agreement is very good between these experimental results and the trends predicted by equation (17).

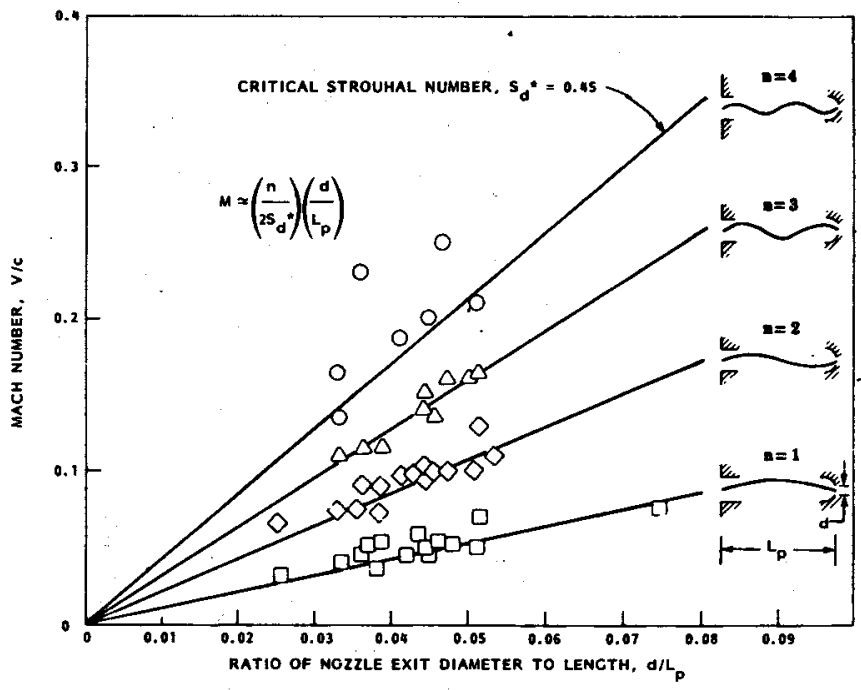

Fig. 19 Relation between Mach number and nozzle length for organ-pipe CAVIJET ${ }^{\circledR}$ nozzles

Effect of Nozzle Geometry on Critical Strouhal Number. Fig. 20 illustrates the nozzle features which are being varied so as to determine their effect on the critical Strouhal number. Fig. 21 shows the response in air for a supply tube of length $8.75 \mathrm{in}$. $(22.2 \mathrm{~cm}), 0=0.81$ in $(20.6 \mathrm{~mm})$, and $d=0.4$ in. (10.2 $\mathrm{mm}$ ) with a nozzle having a fixed inlet contour. The solid and dashed curves are for two different exit contours, and the various symbols are for several throat lengths. It will be noted that the critical Strouhal number may be varied by changing such nozzle features.

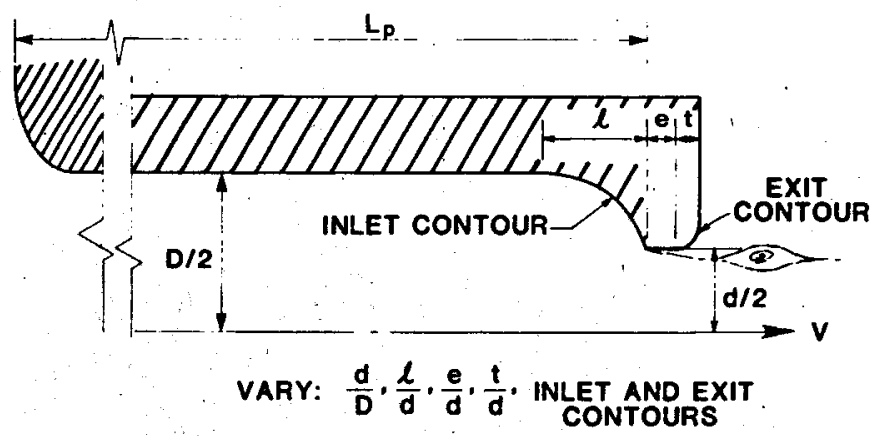

Fig. 20 Organ-pipe nozzle features tested to determine effect on critical Strouhal number

Fig. 22 shows the measured response in water of an organ-pipe supply tube having $L=4.3 \mathrm{in} .(10.9 \mathrm{~cm})$, $D=0.40$ in. $(10.2 \mathrm{~mm}), d=0.2 \mathrm{in} .(5.1 \mathrm{~mm})$, and $a$ nozzle with fixed inlet contour. The different curves are for various throat lengths and exit contours. The critical Strouhal numbers for each nozzle are also shown in this figure. Again it is important to note that there is a substantial variation in the critical Strouhal number as the nozzle features are changed. We have tested nozzles having $S_{d}{ }^{*}$ as $10 w$ as 0.28 and as high as 0.9 . 
Although we have found testing in air to be useful, there are important differences between results obtained in air and in water. These discrepancies were not surprising in view of the seventy-fold difference in Reynolds number.

Response Width and Amplitude. Experlence has shown that the jet is structured into ring vortices whenever the anemometer or pressure signals become periodic even when the magnitude of $u^{\prime} / V$ or $p^{\prime} / \Delta p$ is only 5 to 10 percent. Figs. 21 and 22 show that the width of the response is approximately \pm 15 percent of the Mach number at which the maximum modulation amplitude occurs.

In Figs. 21 and 22 it can be seen that the maximum modulation amplitude decreases as the nozzle features are altered so as to raise the critical strouhal number This result is expected because increasing the Strouhat number corresponds to a reduction in $\lambda$, the spacing between the vortices. Thus the circulation strength, $v \lambda$, of each vortex is lower, and the maximum value of $u ' V$ in the jet should decrease.

It was important to be able to vary the critical Strouhal number by changing the nozzle features, so that self-excited systems could be designed despite constraints on the tube length. However, it should be recognized that when there are no length restraints, nozzles should be selected to have the lowest possible So*, compatible with jet structuring, so that the circulation strength of each vortex is maximized. The lowest Strouhal number for which structured flow has been observed is approximately 0.3 .

Cavitation Inception. The prediction that structured jets should have higher incipient cavitation numbers in comparison to unstructured jets has been confirmed by every test conducted in water. In most cases cavitation was first observed near the boundary,

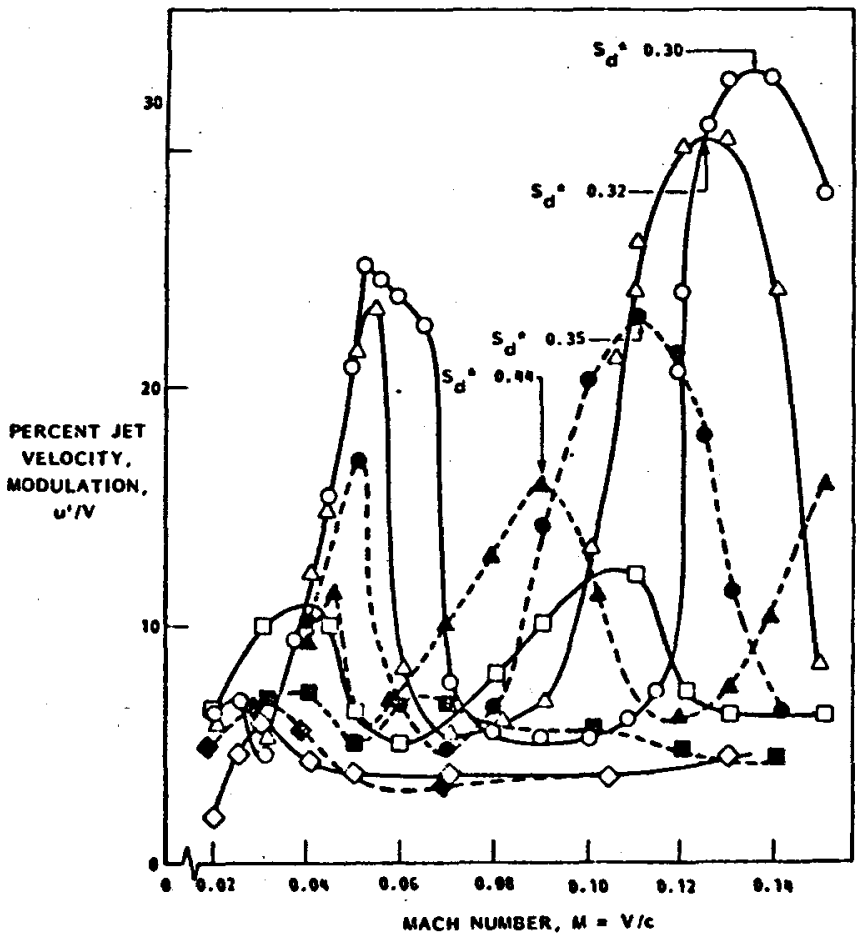

Fig. 21 . Effect of variations in nozzle shape on organpipe CAVIJET ${ }^{\circ}$ resonance in air. Nozzle diameter: $d=0.40$ in.: Feed-tube: curved, in three-cone bit leg, diameter: $D=0.81 \mathrm{in}$.; length: $L_{p}=8.75 \mathrm{in}$. and out several jet radif from the jet axis. Using the curve shown in Fig. 3 for conventional drill bit nozzles as a bench mark for incipient cavitation numbers, the measured incipient cavitation numbers for the self-excited structured jets tested were two to six times higher than this baseline curve. As pointed out previously, almost all of the nozzles being studied had supply pipe to orifice diameter ratios of two or less. Thus the major goal of achieving higher incipient cavitation numbers for nozzles supplied with pipe diameters less than approximately twice the orifice diameter has been achieved.

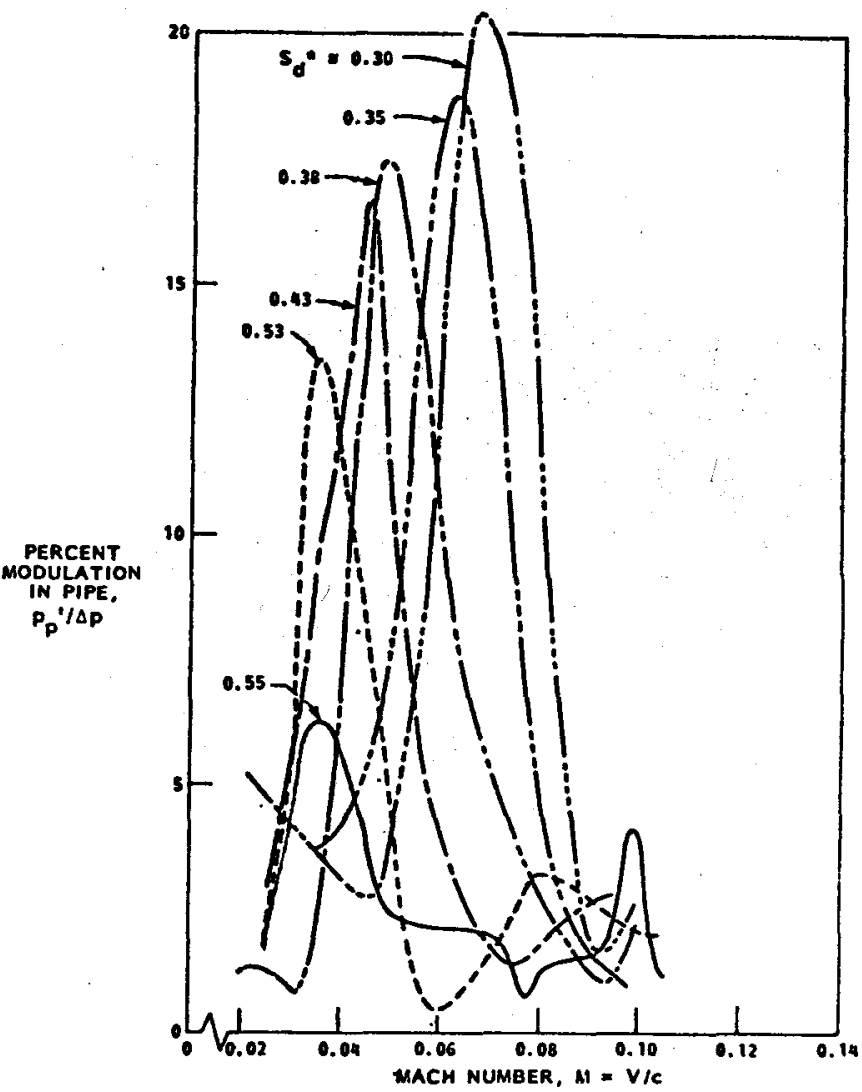

Fig. 22 Effect of variations in nozzle shape on organpipe CAVIJET ${ }^{\oplus}$ resonance in water. Nozzle diameter: $d=0.20$ in.: Feed-tube: straight, diameter: $D=0.40$ in., length: $L_{p}=4.37 \mathrm{in}$.

Although we have not yet carried out tests to demonstrate the low boundary pressures (and improved bottom hole cleaning) that should result when jets are structured, the very high measured incipient cavitation numbers confirm the flow pattern which leads to the boundary pressure predictions.

A further observation has been made relative to cavitation. We have observed that many nozzle shapes are capable of salf excitation when the operating cavitation number is less than the inception value. However, when $\sigma / \sigma_{i}>1$ only a narrow family of nozzle shapes will self excite and structure into vortex rings. We are currently concentrating our studies on this phenomenon.

\section{Rock Cutting Tests}

Although most of our work to date has been directed toward developing procedures for designing self-excited structured jet nozzles to be tried in existing mechan- 
ical bits, we have carried out several tests to compare the rock cutting ability of unstructured and structured cavitating jets. Fig. 23 shows the dimensions of an organ-pipe configuration used to feed either a standard SMITH Tool nozzle shape or a CAVIJET nozzle, each with $d=0.25 \mathrm{in} .(6.4 \mathrm{~mm})$. These nozzles were tested in the High Pressure Cell at various nozzle pressure drops and ambient pressures.

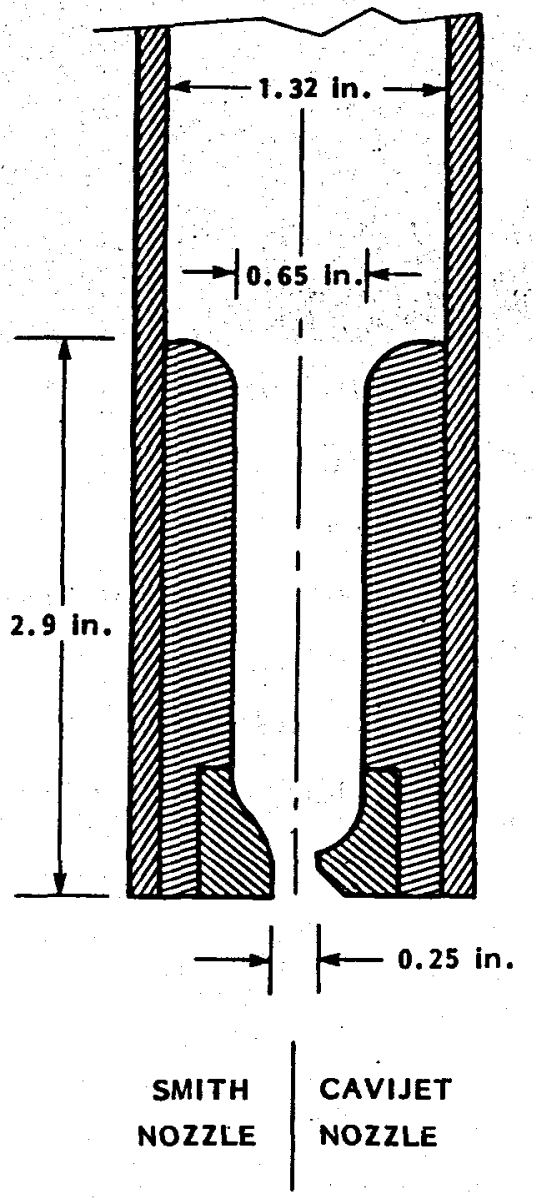

Fig. 23 Configurations of organ-pipe nozzles used in rock cutting tests (See Fig. 24)

A typical comparison is shown in rig. 24 between a self-resonating cavitating nozzle and a conventional SMITH nozzle supplied for use in a roller-cone deephole drill bit. Seen is a top view of one face of a 6 in. $(15.2 \mathrm{~cm})$ cube specimen of Indiana limestone. To allow a half-circle cut to be made by each nozzle, one half of the rock face was protected by a metal plate. The nozzle was then changed, the plate shifted to protect the other half of the cube-face, and the same conditions were run again. In this way, variations due to rock properties could be minimized. Under these conditions, the mean slot depth cut by the ORGAN-PIPE CAVIJET was 4.3 times deeper than that cut by the SMITH nozzle.

Since a major goal of our studies is to design self-excited nozzle systems capable of replacing the nozzle systems of current bit designs, it is necessary to compare the rock cutting performance of candidate new designs with typical existing designs. Fig. 25 shows a comparison of the geometry of an existing design (SMITH) and an early candidate designed to

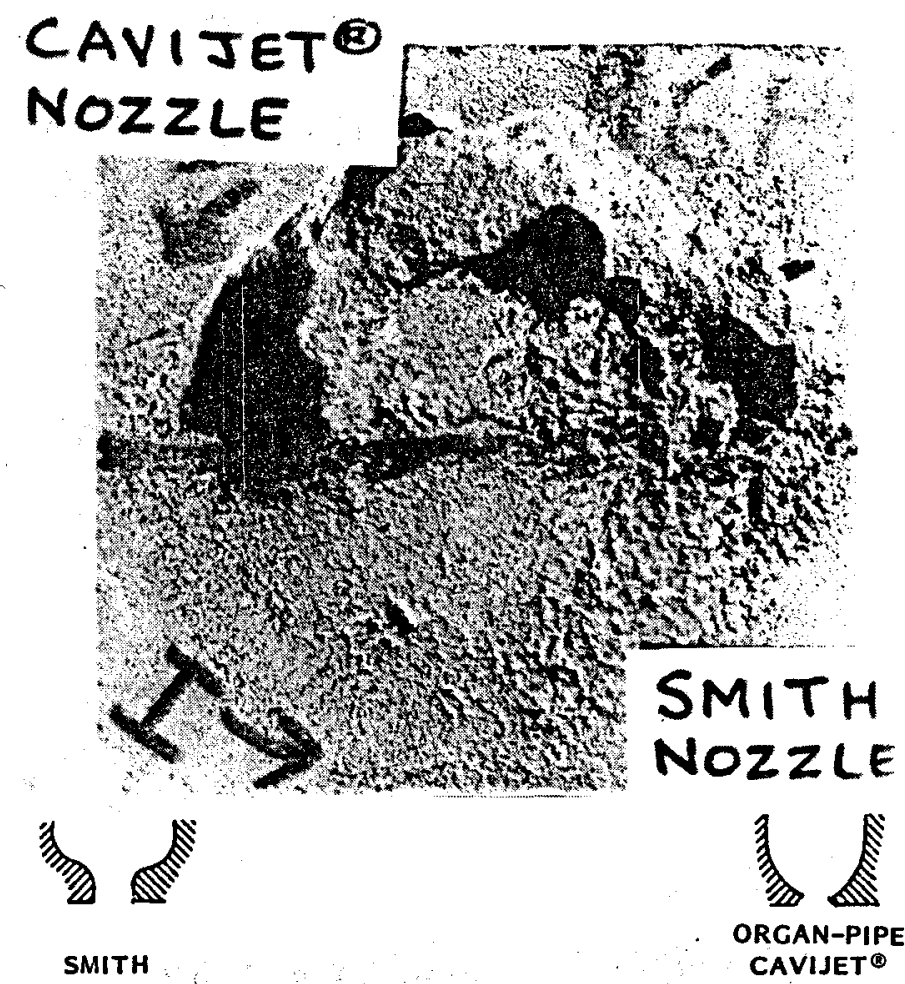

ROCK: Indiana limestone, STANDOFF: $X / d=3$

NOZZLE PRESSURE: $\triangle p=10.3 \mathrm{MPa}(1,500 \mathrm{psi})$

AMBIENT PRESSURE: $p_{\mathrm{a}}=0.83 \mathrm{MPa}(120 \mathrm{psi})$

CAVITATION NUMBER: $\sigma=0.08$

TEST MODE: Single pass in HPC

TRANSLATION VELOCITY: $v=6.4 \mathrm{~mm} / \mathrm{s}(0.25 \mathrm{in.} / \mathrm{s})$

NOZZLE ORIFICE DIAMETER: $d=6.4 \mathrm{~mm}(0.25 \mathrm{in}$.

MEAN SLOT DEPTHS: SMITH $4.3 \mathrm{~mm}(0.17 \mathrm{in.})$

CAVIJET: $18.3 \mathrm{~mm}(0.72 \mathrm{in.})$

Fig. 24 Comparison of slots cut by self-resonating cavitating jet and conventional jet

self excite and structure the jet. The model designs shown are half the full scale dimensions.

Fig. 26 is typical of photographs taken of cavitation which occurs in the ring vortices of the structured jet created by the CAVIJET model. The rings are generally very coherent for about four diameters and are entirely disrupted after about six diameters. Fig. 27 is a photograph which clearly shows a ring vortex spreading over the boundary. The photographs generally confirm that the vortex convection velocity is about two-thirds of the mean jet velocity and thus the vortex spacing is approximately $2 \mathrm{~d} / 3 \mathrm{~S}_{\mathrm{d}}$.

Table 1 presents some rock cutting data for the two nozzles in Fig. 25. Except at the very low cavitation number of 0.067 , the ORGAN-PIPE CAVIJET nozzle system cut this limestone to a depth 2 to 2.5 times greater than the standard SMITH system. It is important to note that for the pressure drops $(<2,500 \mathrm{psi})$ (17.2 $\mathrm{MPa}$ ) currently used in drilling practice, we do not expect the jets to deeply kerf the rock - even with improved nozzle sys tems under cavitating conditions. However, the more intense excited jets should provide enhanced cleaning of the hole battom. The rock cutting results presented here are for traversing rates much less than those used in rotary drilling and serve only as a measure of the relative intensity of various designs. 

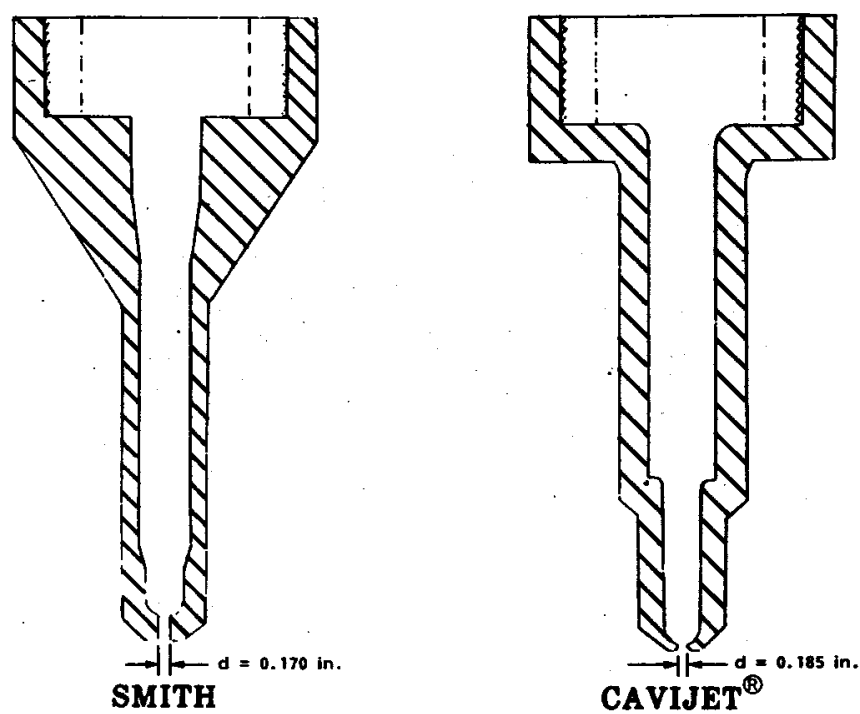

Fig. 25 Half-scale nozzles used in comparative rock cutting tests

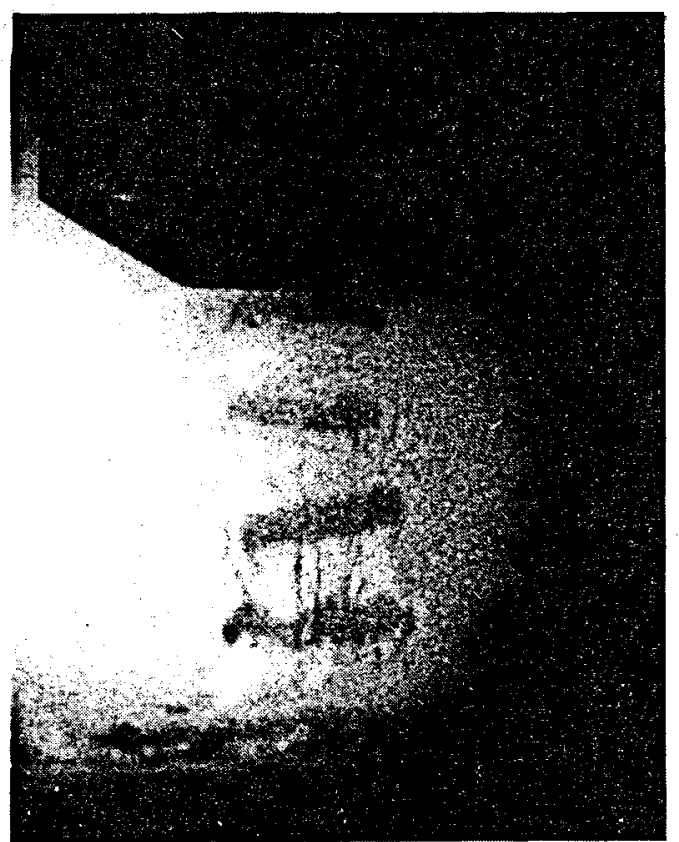

STANDOFF: $X=12.7 \mathrm{~mm}(0.5 \mathrm{in.}) ; X / d=2.7$

NOZZLE PRESSURE DROP: $\Delta p=17.2 \mathrm{MPa}(2,500 \mathrm{psi})$ AMBIENT PRESSURE: $p=3.9 \mathrm{MPa}(560 \mathrm{psi})$

NOZZLE ORIFICE DIAMETER: $d=4.7 \mathrm{~mm}(0.186 \mathrm{in.})$ RESONANCE FREQUENCY: $20.5 \mathrm{kHz}$

STROUHAL NUMBER: $S_{d}=0.52$

CAVITATION NUMBER: $\sigma=0.22$

NOZZLE: ORGAN-PIPE CAVIJET ${ }^{\circ}$, with two-step pipe configuration. This nozzle is half-scale model of CAVIJET ${ }^{\oplus}$ to be used in a threecone roller bit.

Fig. 26 Typical photo of ring vortices form selfresonating CAVIJET ${ }^{\oplus}$ nozzle. Note vortex spreading across surface of target plate

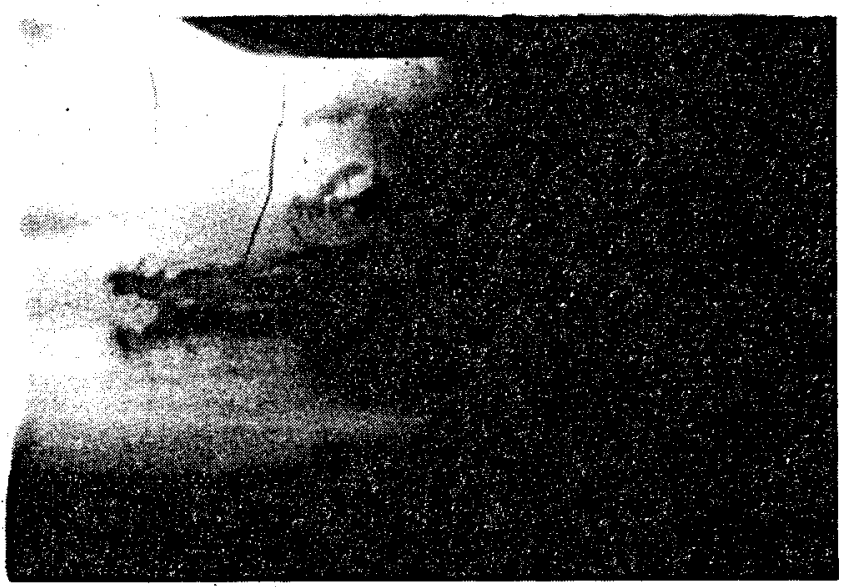

NOZZLE: ORGAN-PIPE CAVIJET

NOZZLE DIAMETER: $d=0.186 \mathrm{in}$.

STANDOFF: $X / d=1.7$

NOZZLE PRESSURE: $\triangle p=1040 \mathrm{p} \$ \mathrm{~s}$

CAVITATION NUMBER: $\sigma=0.30$

RESONANCE FREQUENCY: $f=14.4 \cdot \mathrm{kHz}$

Fig. 27. Photograph of cavitating ring vortices spreading on surface

Table 1 slot cutting comparisons

NOZZLES: Conventional SMITH, $d=0.170 \mathrm{in} .(4.3 \mathrm{~mm})$ ORGAN-PIPE CAVIJET, $d=0.185 \mathrm{in} .(4.7 \mathrm{~mm})$ ROCK: Indiana limestone; STANDOFF: $\mathrm{X} / \mathrm{d}=3$ TRANSLATION VELOCITY: $0.25 \mathrm{in.} / \mathrm{s}(6.4 \mathrm{~mm} / \mathrm{s})$

\begin{tabular}{|l|c|c|c|}
\hline $\begin{array}{c}\text { Pressure, } \\
\text { Nozzle } \\
\text { psi (MPa) }\end{array}$ & $\begin{array}{c}\text { Ambient } \\
\text { Pressure, } \\
p_{\mathrm{a}}, \mathrm{ps} \text { (MPa) }\end{array}$ & $\begin{array}{c}\text { Cavitation } \\
\text { Number, } \\
\sigma\end{array}$ & $\begin{array}{c}\text { Mean Slot } \\
\text { Depth Ratio, } \\
\text { CAVIJET } \\
\text { SMITH }\end{array}$ \\
\hline \multicolumn{4}{|c|}{ SINGLE PASS } \\
\hline $2,530(17.4)$ & $170(1.2)$ & 0.067 & 1.16 \\
$2,500(17.2)$ & $600(4.1)$ & 0.24 & 2.54 \\
$2,500(17.2)$ & $1,200(8.3)$ & 0.48 & 2.33 \\
\hline \multicolumn{5}{|c|}{ TWENTY-FIVE PASSES } \\
\hline $1,225(8.4)$ & $325(2.2)$ & 0.27 & 1.90 \\
\hline
\end{tabular}

\section{PLANNED FUTURE WORK}

In this paper we have summarized our progress to date on a program of investigation which is on-going and far from complete. In order to proceed as rapidly as possible in the development of procedures for designing self-excited, ORGAN-PIPE CAVIJET nozzle systems suitable for installation in existing mechanical bits, we have postponed systematic studies of a number of areas - hence complete understanding of some of our observations is presently lacking. Much research remains to be done and should be done.

At this time the system designs are based on model tests conducted in water, usually at model scales of $1 / 3$ to $1 / 2$. We do not know how drilling mud affects these resuits and plan laboratory tests to gain insights 
into, rheological effects on the phenomena. Such testing will serve either to modffy the nozzles selected from water tests or to help in interpreting field results.

To obtain insights into the effect of the ORGNN-PIPE CAVIJET concept, as presently developed, on drflling rates we have designed systems suftable for installation in a three-cone roller bit. NL Hycalog and the SHITH Tool Company plan to fabricate some of these modiffed bits and test them in the field in 1982.

\section{CONCLUDING REMARXS}

Since any report of a work in progress must contain many unanswered questions, we beg the reader's indulgence and trust that continued efforts now being exerted will allow us to fill in these gaps.

However, several important conclusions are now possible and may be summarized as follows:

1. Using the ORGAN-PIPE CAVIJET concept, selfexcited jets can be obtained which structure themselves into periodic ring vortices. This concept is adaptable for utilization in mechanical bits used in current deephole drilling.

2. ORGAN-PIPE CAVIJET systems have measured incipient cavitation numbers two to six times higher than those obtained for conventional drill bit nozzles. Thus, bits utilizing self-resonating cavitation effects will have drilling rates influenced at depths two to six times greater than with tools now being used.

These increased values of incipient cavitation numbers are consistent with theoretical predictions and give some credence to the possibility that such structured jets should produce improved bottom hole cleaning, even when operating at depths below where cavitation will exist.

3. The measured natural frequencies of the organpipe systems tested correspond to multiples of half wave length standing waves.

4. The response width of self-excited systems is approximately \pm 30 percent of the design nozzle pressure drop.

5. The critical Strouhal number may be varied by changing details of the nozzle geometry. Thus it is possible to design practical organ-pipe systems for many specified operating conditions.

6. Some nozzle designs which self excite under cavitating conditions do not continue to do so under noncavitating conditions. However, designs have been tested which self excite and produce structured jets regardless of the operating cavitation number.

7. There are important differences in the critical strouhal number of nozzles tested in air and water. and it may be anticipated that the Strouhal number might be different in water versus drilling mud. The magnitude of such a rheological effect, if it exists, is not yet known.

\section{ACKNOWLEDGEMENTS}

The work described in this paper was supported in part by the U.S. Department of Energy, Diviston of Geothermal Energy, under Sandia Laboratories Contract Nos. 13-5111 and 13-5129; support by the authors' companies, HYDRONAUTICS, Incorporated and NL HYCALOG/ NL Industries, Incorporated has also been provided for this study.

\section{REFERENCES}

1. Cunningham, R.A. and Eentink, J.G. "Laboratory Study of Effect of Overburden, Formation and Mud Column Pressures on Drilling Rate of Permeable Forma- tions." Petroleum Transactions, AIME, Yol. 216, 1959, pp. 9-17.

2. Maurer, H.C.. "The 'Perfect-Cleaning' Theory of Rotary Drilling," Journal of Petroleum Technology, 1962, PD. 97-101.

3. Feenstra, R. and Van Leeuwen, J.J.M., "Full Scale Experiments on Jets in Impermeable Rock Orilling." Journal of Petroleum Technology, Vol. 231 . March 1964, pp. 329-336.

4. Sutko, A.A., Sifferman, T.R., Haden, E.L.. and Wahl, H.A., "Bit Nozzle Changes Could Improve Drilling Hydraulics," Oil and Gas Jo!rnal. February 17. 1975, pp. 102-106.

5. Pratt, C.A., "Increased Penetration Rates Achieved With New Extended Nozzle Bits,"Journal of Petroleum Technology, Vol. 30, August 1978, pp. TI91Tig8.

6. Johnson, V.E., Jr., et al., "Tunnelling, Fracturing, Drfliting and Mining With High Speed Water Jets Utilizing Cavitation Damage," Proc. First Int'1. Sympos. on Jet Cutting Technology (Coventry, U.R.: April 5-7, 1972) Cranfield, U.K., BHRA Fluid Engineering, Paper A3, 1972, 19 pp.

7. Conn, A.F. and Johnson, Y.E., Jr., "Further Applications of the CAVIJET" (Cavitating Water Jet) Method," Proc. Second Int'1. Sympos. on Jet Cutting Technology (Cambridge, U.K.: April 2-4, 1974) Cranfield, U.K., BHRA Fluid Engineering; Paper D2, 1974. $14 \mathrm{pp}$.

8. Conn, A.F. and Rudy, S.L., "Cutting Coal With the CAVIJET" Cavitating Water Jet Method," Proc. Third Int'1. Sympos. on Jet Cutting Technology (Chicago, U.S.A.: May II-13, 1976) Cranfield, U.K., BHRA Fiuid Engineering, Paper D8, 1976, 9 pp.

9. Conn, A.F.. and Rudy, S. L., "Conservation and Extraction of Energy With the CAVIJET"," Proc. Fourth Int' 1 . Sympos. on Jet Cutting Technology (Canterbury, U.K.: April 12-14, 1978) Cranfield, U.K. BHRA Fluid Engineering, Vol. 1, Paper H2, 1978, 20 pp.

10. Conn, A.F. and Johnson, V.E., Jr., "The Fluid Dynamics of Submerged Cavitating Jet cutting," Proc. Fifth Int'1. Sympos. on Jet Cutting Technology (Hanover, F.G.R.: June 2-4, 1980), Cranfield, U.K. BHRA Fluid Engineering, Paper Al, 1980, pp. 1-14.

11. Leinhard, J.H. and Stephenson, J.M. "Temperature and Scale Effects Upon Cavitation and Flashing in Free and Submerged Jets," Journal of Basic Engineering, Trans. ASME, Series D, Vol. 88, 1966, pp. 525-532.

12. Conn, A.F. Johnson, V.E., Jr. Liu, H.L. and Frederick, G.S. "Evaluation of CAVIJETO Cavitating Jets for Deep-Hole Rock Cutting," HYDRONLAUTICS, Incorporated Technical Report 7821-1, August 1979 (A1 so Sandia Laboratories Contractor Report SANDBi7067. May 1981).

13. Crow, S.C. and Champagne, F.H., "Orderly Structure in Jet Turbulence," Journal of Fluid Mechanics, Vo1. 48, Part 3, August 1971, Pp. 547-591. 
14. Johnson, V.E., Jr. L Lindenmuth, H.T., Conn, A.F., and Frederick, G.S., "Feasibility Study of Tuned-Resonator. Puisating Cavitating Water Jet for Deep-Hole Drilling," HYDRONAUTICS, Incorporated Technical Report 8001-i, May 1981 (also Sandia Laboratories Contractor Report SAND81-7126, August 1981).

15. Kinsler, L.E. and Frey, A.F., Fundamentals of Acoustics, John Wiley and Sons, Inc., Kew York, 1962.

16. Wylie, E.B. and Streeter, V,L.., Fluid Transients, McGraw Hill Inc., New York, 1978.

\section{APPENDIX A}

\section{A PRELIMINARY CHIP-LIFTING ANALYSIS}

Fig. 13 illustrates a chip of characteristic size, $d_{c}$. being acted on by the instantaneous boundary pressure, $\mathrm{Pb}$, nomally being held down by the overpressure, 6 . 1.e., the difference in the ambient pressure, $p_{a}$, and the pore pressure, $\mathrm{Pp}_{\mathrm{p}}\left(\hat{\mathrm{p}}=\mathrm{p}_{\mathrm{a}}-\mathrm{pp}_{\mathrm{p}}\right)$. It is of interest to first neglect the hydrodynamic drag on the particle as it rises when acted on by the pressure difference, $\Delta p_{c}=p_{p}-p_{b}$. is:

The acceleration of the chip (neglecting buoyancy)

$$
a=\frac{\Delta p_{c}}{\left(\frac{\rho_{m}}{\rho}+c_{m}\right)},
$$

where $C_{m}$ is the added mass coefficient, $O_{m}$ is the chip density, and $p$ is the liquid density.

The approximate time that the pressure $\mathrm{pb}$ acts on the chip, from Fig. 12, is (for the assumed $\lambda / d \simeq 1$ ):

$$
t \simeq w / d f \text {. }
$$

where $f$ is the frequency of vortex passage. The distance, $l$, moved by the chip is then

$$
l=t a t^{2}=\frac{\Delta p_{c}}{2\left(\frac{p_{m}}{\rho}+c_{m}\right)} \cdot\left(\frac{w}{d f}\right)^{2}
$$

Now,

$$
P_{a}-P_{b}=P_{p}+\hat{p}-P_{b}=K \Delta p
$$

where $\Delta p=$ nozzle pressure $d r o p=\frac{1}{2} p V^{2}$, and $K$ is given by equation (16).

Therefore

$$
\Delta p_{c}+\hat{p}=K \Delta p
$$

and

$$
\Delta p_{c}=K \Delta p-\hat{p}
$$

Equation (21) may then be written as

$$
\frac{l}{d_{c}}=\left(\frac{w}{d}\right)^{2}\left(\frac{v}{f d}\right)^{2} \frac{\left(k-\frac{\hat{p}}{\Delta p}\right)}{4\left(\frac{p_{m}}{\rho}+c_{m}\right)}\left(\frac{d}{d_{c}}\right)^{2}
$$

From equation (15) or Fig. 12, take $K=10$, $w / d=0.15$, Strouhal number: $S_{d} \simeq 0.5, \rho_{m} / \rho \simeq 2.5$, and $C_{m}=1$. Then equation (24) becomes

$$
\frac{l}{d_{c}}=0.0064(10-\hat{p} / \Delta p)\left(\frac{d}{d_{c}}\right)^{2}
$$

If we take $\hat{p} / \Delta p=1$, for example $\hat{p}=\Delta p=1,500$ psi $(10.3 \mathrm{MPa})$, then equation (25) shows that $\mathrm{dc} / \mathrm{d}$ $=0.24$ for $2 / d_{c}=1$. This means that chips smailer than $0.24 \mathrm{~d}$ should be lifted distances equal to or greater than their characteristic dimension.

Consider a fairly high drilling rate of $50 \mathrm{ft} / \mathrm{hr}$ $(15.2 \mathrm{~m} / \mathrm{hr})$. within a range of rotary speeds of 60 to $120 \mathrm{rpm}$. For a drilling rate of $50 \mathrm{ft} / \mathrm{hr}(15.2 \mathrm{~m} / \mathrm{hr})$ the average chip size in this range of typical rotary speeds is 0.08 to $0.16 \mathrm{in}$. ( 2 to $4 \mathrm{~mm}$ ) or less. In a $12 x_{6}$ in. $(31.1 \mathrm{~cm})$ three-cone roller bit, the nozzle diameters are typically $0.4 \mathrm{in.}(10 \mathrm{~mm})$. Thus, to achleve a $50 \mathrm{ft} / \mathrm{hr}(15.2 \mathrm{~m} / \mathrm{hr})$ drilling rate, the maximum value of $d_{c} / d$ to be removed ranges from about 0.2 to 0.4 . For the example given, the smaller of these chip sizes would be lifted compleiely out and the larger more than half way out. This would seem to be an adequate lift to assure their total removal. Equation (19) assumes the chip is retarded only by inertia. This is clearly not true since the velocity of the chip, $v$, after the vortex passes must.be: $v=$ a $t$, or:

$$
\frac{v}{v}=\frac{(K-\hat{p} / \Delta p)}{\left(2 \frac{p_{m}}{p}+c_{m}\right)}\left(\frac{w}{d}\right)\left(\frac{v}{f d}\right)\left(\frac{d}{d c}\right)=\frac{2\left(\frac{l}{d c}\right)\left(\frac{d}{d}\right)\left(\frac{f d}{v}\right)}{\frac{w}{d}}
$$

For the conditions taken in the example following equation (24), $v / V=1.6$. It is not likely that the drag associated with such high velocities is neligible. The ratio of the drag force associated with this velocity to the vortex lifting force is approximately

$$
\frac{F_{\text {drag }}}{F_{\text {vortex }}}=\frac{(v / v)^{2}}{k-\frac{p}{\Delta p}}
$$

Fdrag

For the example given, $\frac{\text { Frag }}{\text { Fortex }}=0.28$. That is, neglecting the drag force does not greatly affect the results given by equation $(25)$, particularly if $\ell / d_{c}$ $<1$.

The worst possible situation that has been postulated in the chip hold down problem exists for impermeable formations where a chip is formed and the crack beneath the chip is assumed to be a vacuum; that is, $\hat{\sigma}$ is assumed to be equal to $\mathrm{Pa}$. Then equation (24) becones:

$$
\left(\frac{l}{d_{c}}\right)_{\text {vacuum }}=\left(\frac{w}{d}\right)^{2}\left(\frac{v}{f d}\right) \frac{\left(\dot{K}-\frac{p_{a}}{\Delta p}\right)}{4\left(\frac{\rho_{m}}{\rho}+c_{m}\right)}\left(\frac{d}{d_{c}}\right)^{2}
$$

or

$$
\frac{\left(\frac{l}{d_{c}}\right)_{p_{p}}=0}{\left(\frac{l}{d_{c}}\right)_{p_{p}}}=\frac{K-\frac{p_{a}}{\Delta p}}{K-\frac{p}{\Delta p}}
$$

Using the previous example case: $K=10, \Delta p=\hat{p}=-2,000$ psi (13.8 MPa), but assuming $\mathrm{Pa}$ is $10,000 \mathrm{psi}(69.0 \mathrm{MPa})$ in a deep well, the chip in the worst case would be lifted $5 / 9$ of the values given previously. This reduced value may still be sufficient to allow the jet lateral drag to remove the chip.

All of the foregoing discussion fails if the theoretical value of $K$ is greatly reduced in nature by viscous effects. However, it must be reduced by an order of magnitude in order to become of no significance in cleaning the hole bottom. We are continuing our studies to learn more about this effect. 
HYDRONAUTICS, Incorporated

\author{
APPENDIX B \\ "Self-Resonating Cavitating Jets" \\ Presented at the \\ 6th International Symposium on \\ Jet Cutting Technology \\ Surrey, England \\ Apri1 6-8, 1982
}




\title{
Jet Cutting Technology
}

6-8, April, 1982

\section{SELF-RESONATING CAVITATING JETS}

\author{
V.E. Johnson, A.F. Conn, W.T. Lindenmuth
}

G. L. Chahine and G.S. Frederlck

Hydronautics, Incorporated, U.S.A.

\begin{abstract}
Summary
Cavitation has been shown to provide substantial enhancement to the performance of jets used for cutting and cleaning applications. A further improvement to such jets can be obtained if the flow is caused to pulsate at frequencles corresponding to the predominant frequency of the structures in the jet shear layer. Several passive methods to enable these jets to resonate are now being developed. It should be emphaslzed that no external means or moving parts are utllized. The pulsations are achleved by self-sustaining osclllations within specially designed nozzles and supply piping. One design, the "PULSER CAVIJET ß", consists of tandem orifices, with a resonating chamber in between. Another concept, the "ORGANPIPE CAVIJET B", causes pressure amplifications by means of standing waves in the plpe leading up to the nozzle.

The research leading to the development of these new types of cavitating jets is discussed, including descriptions of the experimental methods used to study their performance. Comparative testing has shown that self-resonating cavitating jets can cut rock and perform underwater cleaning more effectively and faster than elther conventlonal jets or nonresonating cavitating jets.
\end{abstract}


NOMENCLATURE

\begin{tabular}{|c|c|c|c|c|c|}
\hline c & speed of sound in fluid & $\mathbf{L} / \mathbf{T}$ & $\Gamma$ & circulation & $F^{2} / T$ \\
\hline d & nozzle diameter & $\mathbf{L}$ & $\delta$ & boundary layer thickness & $\mathbf{L}$ \\
\hline D & diameter of pipe & $\mathbf{L}$ & $\lambda$ & acoustic wave length & $\mathbf{L}$ \\
\hline $\mathbf{f}$ & frequency & $T^{-1}$ & y & kinematic viscosity & $L^{2} / T$ \\
\hline $\mathbf{K}_{\mathbf{n}}$ & mode parameter & ND & $\rho$ & fluid density & $F T^{2} / L^{4}$ \\
\hline$\ell, L$ & length & $\mathbf{L}$ & $\sigma$ & cavitation number & ND \\
\hline $\mathbf{M}$ & Mach number, V/c & ND & SU & SCRIPTS & \\
\hline $\mathbf{n}$ & mode number & ND & $\mathbf{a}$ & ambient & \\
\hline $\mathbf{N}$ & Integer & ND & c & vortex core; centerbody & \\
\hline $\mathbf{p}$ & pressure & $F / L^{2}$ & d & see Figure 9 & \\
\hline$\Delta \mathrm{p}$ & nozzle pressure drop & $F / L^{2}$ & 1 & Incipient, Induced & \\
\hline $\mathbf{r}$ & radius & $\mathbf{L}$ & $\mathbf{f}$ & feed-pipe & \\
\hline $\mathbf{R}_{\mathbf{e}}$ & Reynolds number, Vd/v & ND & 0 & initial value, jet exiting & value \\
\hline$S_{d}$ & Strouhal number, $S_{d}=f d / V$ & ND & $\mathbf{s}$ & $\begin{array}{l}\text { plpe } \\
\text { source pipe }\end{array}$ & \\
\hline $\mathbf{t}$ & time & $\mathbf{T}$ & $t$ & see FIgure 8 & \\
\hline $\mathbf{u}^{\prime}$ & excited fluctuating velocity & $\mathrm{L} / \mathrm{T}$ & $\mathbf{v}$ & vortex, vapor pressure & \\
\hline $\mathbf{V}$ & jet velocity & $\mathrm{L} / \mathrm{T}$ & SU & ERSCRIPTS & \\
\hline$\forall$ & volume & $\mathbf{L}^{3}$ & ' & rms of fluctuating componen & nt \\
\hline $\mathbf{X}$ & $\begin{array}{l}\text { distance from orifice } \\
\text { distance from surface }\end{array}$ & $\mathbf{L}$ & * & $\begin{array}{l}\text { critical Strouhal number, } \\
\text { optimum jet structuring }\end{array}$ & for \\
\hline
\end{tabular}




\section{INTRODUCTION}

\subsection{Motivation}

During the past two years our studies of cavitating jets have concentrated primarily on new ways to improve their cutting and cleaning action. The emphasis of these efforts has been on submerged jets, operating at large cavitation numbers, $\sigma$; typical1y: $\sigma=0.5$ to 2 (where: $\sigma=\mathrm{pa} / \Delta \mathrm{p}, \mathrm{p}_{\mathrm{a}}$ : amblent pressure, and $\Delta \mathrm{p}$ : pressure drop across the nozzle). The main motivation for this work is a desire to develop jets capable of effectively enhancing the performance of mechanical drill-bits at greater depths than now possible with our conventional CAVIJET cavitating jets.

Several papers have described our earlier efforts with what are now being called "conventional" cavitating jets (Refs. 1 to 6), to distinguish them from the "selfresonating" cavitating jets which are the topic of this paper. Results from laboratory and field trials have shown that the cavitation erosion will weaken or remove rock, thus facilitating the action of the mechanical bits. Our initial studies involved efther "plain" CAVIJET nozzles, 1.e., merely a nozzle-body designed to enhance cavitation with no Inserts, or a "centerbody" CAVIJET, where the centerbody Insert was usually a flat-ended circular cylinder having a diameter equal to one-half the diameter of the nozzle orifice.

To be effective at greater depths, the self-resonating cavitating jet nozzle should have a larger cavitation number at inception, $\sigma_{1}$, relative to that of the conventional jet, $\sigma_{1,0}$. If, for example, a nozzle is operated at a fixed $\Delta p$, then $p_{a}$ must decrease below, a limit value, $\sigma_{1} \Delta p$, for cavitation to begin to occur. In deep hole drilling this means that, depending on the density of the surrounding fluid, the depth has to be smaller than a certain value. Thus, for an operating $\sigma<\sigma_{1}$ (shallower depths) there will be cavitation; for $\sigma>\sigma_{1}$ (greater depths), cavitation will be suppressed. It has been demonstrated that a nozzle having a larger $\sigma_{i}$ will remain erosive to a greater depth (or $\mathrm{p}_{a}$ ), and at any depth will be more erosive than a nozzle with a smaller $\sigma_{i}$. By causing the jet flow to pulsate or oscillate, higher instantaneous driving pressures are available to collapse cavities - thus intensifying the erosive process.

In deep-hole drilling, rock cuttings fractured by the mechanical tool action must be rapidly cleared from the bottom, or they will be reground by subsequent cutters. Without removal of these chips (see, for instance, Maurer, Ref. 7), the drilling capability of a deep-hole bit is greatly reduced. The localized pressure fluctuations created by submerged, self-resonating jets provide an additional mechanism for increasing drilling rates, by serving to improve the process by which rock cuttings are lifted from the hole bottom. The differential pressures tending to hold the chips against the hole bottom, if overcome by the oscillating pressures of these new cavitating jets, would thus allow the washing action of the flow to more readily carry the chips away.

\subsection{Background}

Many Investigators have examined phenomena related to fluctuating jets, either self-resonating or with an external means for stimulating or driving the pulsations in the jet flow. Schematics of several concepts available for pulsing jets are shown in Fig. 1. Crow and Champagne (8) examined structuring of an air jet, using a loudspeaker for excitation (FIg. 1a). When pulsing was provided at a frequency, $f$, which corresponds to a critical strouhal number* of 0.3 (or a multiple of 0.3 ), discrete ring vortices were created and maximum jet velocity, oscillations were observed.

Moore (9) and Kibens (10) have conducted similar research. Morel (11) examined the passive oscillations of a Helmholtz tandem-orifice resonant air jet (Fig. le) at low Mach numbers $(<0.1)$. The advantages of driving a pulsed jet at acoustic resonance frequencles was examined theoretically by Wylle (12), and more detalled discusstons can be found in Ref. 13.

A number of studies, using various mechanical valves (F18. Ib) or other devices to physically interrupt a jet, have shown the efficacy of delivering a series of

* The Strouhal number, $S_{d}$, based on nozzle diameter, $d, 1 s: S_{d}=f d / V_{0}$, where $v_{0}$ is the fet exiting velocity, and $f$ is the frequency of oscillation. 
waterhammer stresses to a surface (see, for Instance Refs. 14, 15, 16) for cleaning or cutting purposes. None of these approaches, however, are operable at the frequencles (see the next section) required to achleve the jet structuring which was the objective of our investigation.

Galle and Woods (17) describe several methods for oscillating the pressure at the bottom of a drilled hole. However, the various fluid amplifier concepts they proposed (see Fig. 1c, for example) are only capable of frequencles several orders of magnitude lower than required to structure a jet at a practical value of $V_{0}$. Fülöp and Rókár (18) suggested a "fluid whistle" concept, for incorporation in deep-hole drills, to cause rock-weakening by creating pressure fluctuations at the natural frequency of the rock being drilled. No considerations of jet-structuring were cited by Fülöp and Rókár; however, a passive device (see Fig. le) of this general type has been examined and is discussed below. Indeed, such "bird whistles", or "Helmholtz resonators" have been discussed and understood for over a century $(19,20,21)$, and were recently reviewed, along with other related self-sustaining oscillating flows, by Rockwell and Naudascher (22).

In summation, a variety of concepts for causing jet fluctuations have been examined, both for air jets and for liquid jets in air or submerged, and much of the basic phenomena of jet resonance have been recognized. None of these previous investigations, however, were seeking the objective pursued in the present study - namely the creation of explicit nozzle design procedures for high velocity submerged fluld jets where passive resonance could be exploited to increase the cavitation erosion intensity of such jets. Although not within the scope of this paper, the potential of selfresonance to create pulsed water jets for in-air cutting and cleaning purposes is also being examined.

\section{JET-STRUCTURING MECHANISMS}

A schematic suggesting the mechanism leading to a series of idealized ring vortices in the shear zone of a submerged jet is shown in Fig. 2. The flow leaves the nozzle, with orifice diameter, $d$, at a velocity, $v$. This velocity is assumed to be uniform over the nozzle exit plane except in the boundary layer region, which is of characteristic thickness, $\delta$. In the upper portion of Fig. 2, an ideal shear zone, having no mixing with the surrounding fluid; is shown. In a real flow, exterior fluid is entrained, and vortices form as shown in the lower portion of this figure. If we assume that the cores of these vortices are made up of segments of the boundary fluid, spaced a distance, $\lambda$, apart, where $\lambda$ is the distance between two vortices, then the cross-sectional area of each core is approximately $\lambda \delta$. If the cores are assumed clrcular, then the core radil, $r_{c}$, are given by:

$$
r_{c}^{2} \simeq \frac{\lambda \delta}{\pi}
$$

With these assumptions, the circulation, $\Gamma$ :

$$
r \equiv \phi v \mathrm{ds}
$$

of the vortex is equal to that circulation in the segment of shear layer of length $\lambda$ :

$$
\Gamma=\lambda v_{0}
$$

If we use a Rankine model for the vortex, it can be shown that the pressure in the center of the vortex, $P_{m i n}$, is given by:

$$
\frac{p_{a}-p_{m i n}}{l_{2} \rho V_{0}^{2}}=\frac{\Gamma^{2}}{2 \pi^{2} V_{0}{ }^{2} r_{c}^{2}} \text {. }
$$

Inception occurs when $p_{\text {min }} \simeq p_{v}$ (where $p_{v}$ is the vapor pressure of the $f 1 u 1 d$ ), then:

$$
\sigma_{1}=\frac{\Gamma^{2}}{2 \pi^{2} v_{0}^{2} r_{c}^{2}}
$$

and combining [1], [2], and [4]:

$$
\sigma_{1}\left(\text { at vortex center) } \simeq \frac{\lambda}{2 \pi \delta}\right.
$$

* See a standiad hyurodynamics text tor properties of Rankine vortices, e.g., Ref. 23. 
To cause increased cavitation and erosion, It is desirable to have $\sigma_{1}$ as large as possible. This can be achieved, as suggested by Eq. [5], efther by Increasing $\lambda$, by decreasing $\delta$, or both. Fọr a given nozzle, jet fluid, and speed, the value of $\delta$ will be fixed. The spacing, $\lambda$, for a structured, resonating jet has been found to be of the order of the jet diameter, d, as shown schematically in Fig. 3a. In contrast a distribution of turbulent eddy sizes will be formed for an unstructured jet, and the mean scale 15 more nearly of the order of $\delta$ than of $d$ (F1g. $3 \mathrm{~b}$ ).

The foregoing discussion did not examine the effects of a boundary upon which the jet is impinging, i.e., the surface being cleaned or eroded. The flows for an unstructured and a structured jet, striking a surface which is located at a standoff distance, $X$, from each nozzle, are contrasted in F1g. 4. As the ring vortex nears the surface, the ring radius, $r_{v}$, will increase. This "stretching" of a vortex is known to cause a decrease in the core s1ze, $r_{c}$. Thus, from Eq. [4], this vortex core decrease is seen to create an increase in $\sigma_{1}$. Therefore, as the boundary is moved near to a cavitating jet, cavitation is first observed adjacent to the boundary. A combination of these mechanisms has been shown experimentally (see Sect. 5.1) to provide inception numbers for structured jets which are two to four times those of the same fet when resonance is not present.

\section{EXPERIMENTAL FACILITIES}

Two facillties were developed to study the characteristics of self-resonating jets. An air facility allows examinations of jet performance with the capability of rapid and economical changes in a design iteration that are not so readily made in the highpressure water jet factlity. Although the existing air facility has been operated at Reynolds' numbers which are too low to allow complete scaling to the water results, valuable insights which have guided and minimized the more tedlous tests in water were gained by studies of resonating alr jets.

\subsection{Air Facility}

A schematic of the components contained in this facility are shown in Fig. 5. The rectangular plenum supplied air to the nozzle being tested. Pressure in the plenum was controlled by a needle valve in the $0.2 \mathrm{MPa}$ supply line from the air compressor, and this pressure was monitored by a U-tube manometer. Both free jet and impinging. jet tests were run, with a movable target plate placed normal to the fet axis at various locations. Perturbations in the jet axial velocity were surveyed with a $25 \mu \mathrm{m}$ diameter hot $\mathrm{film}$ sensor. This probe was mounted on a motor driven rig, allowing a continuous survey along either the axial or radial directions. A nearby microphone monitored acoustic signatures from the nozzle.

The electronic signal from the hot film sensor was conveyed to a Thermo-Systems Model 1050 hot-film anemometer bridge. The output from this bridge was then fed directly to an RMS voltmeter, a spectrum analyzer (Unigon Model 256), and an X-Y plotter. By also sending the RMS voltmeter output to the plotter, side-by-side comparisons could be graphed of the mean, $v$, and fluctuating, $u$ ', velocities. Output from the spectrum analyzer was viewed on an oscilloscope, to allow identification of the resonant frequency peaks in the fluctuating velocity. The microphone output was also fed to an RMS voltmeter and to the spectrum analyzer, thus providing a monitor of the frequency and intensity of sound pressure levels. These components were also used to process the signals from the pressure probes used in the water tests.

\subsection{High Pressure Cell (HPC)}

The test chamber used to observe the behavior of submerged cavitating jets is shown in Fig. 6. This cell can contain an internal pressure of up to $20.7 \mathrm{MPa}(3,000$ psi). This cell ambient pressure is controlled by a "choke", a floating double-ended piston valve, which is balanced by nitrogen gas. This floating action allows for the escape of particles during rock cutting trials. Rock specimens, typically cubes 15.2 $\mathrm{cm}$ ( $6 \mathrm{in.}$ ) on a s1de, can be rotated beneath the jet in the HPC, at rates up to $66 \mathrm{rpm}$ by means of the variable speed drive:

The high pressure fluid for this cell is usually provided by either a three or a five-plunger pump; if a test requires higher flows then both pumps are run in parallel. The triplex pump, which is also rated for drilling mud, will deliver $303 \mathrm{l} / \mathrm{m}$ ( $80 \mathrm{gpm}$ ) at $13.8 \mathrm{MPa}(2,000 \mathrm{psi})$. The special quintuplex pump, with changeable plungers and head, can be run over a range; highest flow: $341 \mathrm{l} / \mathrm{m}(90 \mathrm{gPm})$ at $17.2 \mathrm{MPa}(2,500 \mathrm{ps} 1)$, 
or highest pressure: $68.9 \mathrm{MPa}(10,000 \mathrm{ps})$ with a $76 \mathrm{l} / \mathrm{m}$ (20 gpm) flow capacity.

For flow visualization and photography, the HPC has been fitted with three circular viewing ports, each $3.8 \mathrm{~cm}(1.5 \mathrm{in.})$ in diameter. A typical photograph of a selfresonating cavitating jet taken in this cell is shown in Fig. 7. This photograph was taken by darkening the room, opening the camera shutter, and releasing a single flash from a stroboscopic light source. When observing the jets by eye, the strobe is operated at a suitable submultiple of the jet resonance frequency so as to reasonably "stop" the motion of the vortices. In this manner, jet structure can easily be monitored while operating parameters are varied.

Two piezoelectric pressure transducers (PCB Piezotronics Model No. 101A04; $6.4 \mathrm{~mm}$ dia.) are mounted in the HPC. One is placed in the inlet supply pipe feeding the nozzle; the other is in the wall of the main pressure vessel. As noted above, the same instrumentation used for the air tests serves to process the signals from these pressure transducers.

\section{SELF-RESONATING JET CONCEPTS}

The self-resonating submerged cavitating jets which have been studied can be grouped into three types. These have been given the descriptive names: "PULSER

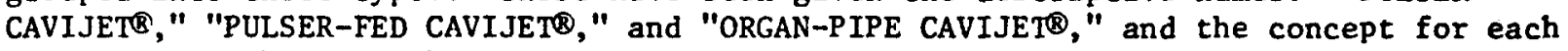
type will now be described.

\subsection{PULSER CAVIJET}

The configuration for this self-resonating jet concept, a tandem-orifice Helmholtz resonator, is shown in Fig. Ea. The steady, high-pressure flow enters through the feed line, diameter $D_{f}$, and passes through an entrance section of length, $L_{S}$, and diameter, $D_{S}$. The flow then contracts through the first orifice, $d_{1}$, passes through the chamber (volume: $\forall$; length, $L$; diameter: $D_{t}$ ) and exits through the second orifice, $\mathrm{d}_{2}$.

If operated at its optimum Strouhal number, $S_{d}=f d_{1} / v_{1}$, discrete ring vortices will be formed in the jet issuing from orifice $d_{1}$. When a vortex arrives at the second orifice, $d_{2}$, a distance $L$ away from $d_{1}$, a pressure signal will be transmitted upstream, arriving back at $d_{1}$ after a time: $t_{L}=L / c$, where $c$ is the speed of sound in the fluid. If the length, $L$, is selected as:

$$
\mathrm{L}=\mathrm{N} \lambda-\mathrm{t}_{\mathrm{L}} \mathrm{V}_{\mathrm{c}}
$$

where: $\mathrm{N}$ is an integer number of vortices, and

$v_{c}$ is the vortex convection velocity, $v_{c}=f \lambda$, then the pressure signal will arrive at $d_{2}$ at exactly the time required to excite a new vortex. Using the expressions for $S_{d}$ and $t_{L}$, and introducing the Mach number: $M=V_{2} / c$, Eq. [6] can be rewritten nondimensionally as:

$$
\frac{L}{d_{1}}=\frac{N\left(V_{c} / V\right)}{s_{d}\left(1+M N_{c} / V_{1}\right)} \text {. }
$$

For applications where a space limitation exists, the so-called "LAID-BACK" PULSER CAVIJET, shown in Fig. $Q b$, might be used. In this concept, part of the necessary resonant chamber volume is contained in the region which surrounds the main flow passage leading to the first nozzle.

\subsection{PULSER-FED CAVIJET}

A self-resonating nozzle concept, which has been shown to be capable of providing several advantages relative to the PULSER CAVIJET, is shown in Fig. 9. Shown are a basic design, Fig. $9 a$, and two designs $(9 b, 9 c)$ with alternative diffusion chambers. In this concept the exit nozzle, $d_{3}$, is fed a fluctuating excitation by the same tandem-orifice with intervening resonant chamber configuration as described in Sect. 4.1.

The advantages of the PULSER-FED CAVIJET concept have been shown to be:

a. The jet formed by the exit nozzle, $d_{3}$, has a more uniform velocity distribution, and the vortices formed here are more cleanly defined.

b. The PULSER $\left(d_{1}\right)$ nozzle can be selected to operate at a strouhal number higher 
than that of the exit ( $\left.d_{3}\right)$ nozzle. Th1s Implies that the resonant chamber pressure can be higher than $P_{2}$, the amblent pressure surrounding the jet exiting from $d_{3}$, and the velocity in the chamber less than $V_{0}$. Therefore, the cavitation number in the chamber will be much higher than that of the exiting jet, and cavitation in this chamber can be avoided.

c. The diffusion chamber $\left(L_{d}, D_{d}\right)$ may be designed to enhance the amplitude of modulation provided by the PULSER chamber.

Disadvantages of the PULSER-FED concept Include:

a. A more complex mechanical configuration, and

b. The overall energy loss, caused by losses in the diffusion chamber, is greater than for the PULSER CAVIJET concepts.

The latter problem can be minimized by using the alternative diffusion chambers shown in Figs. $9 b$ and $9 c$.

\subsection{ORGAN-PIPE CAVIJETO}

A self-resonating fet concept which offers the simplest design, and seems to have considerable promise for being adaptable to existing deep-hole drill bits is shown in Fig. 10. This concept should achleve peak acoustic resonance when a standing wave forms in the "organ-pipe" section (length: $L_{p}$, diameter: D). This section is created by the upstream contraction, $\left(D_{s} / D\right)^{2}$ and the nozzle contraction, (D/d) ${ }^{2}$. Peak resonance will occur when the frequency of the organ-pipe wave is near the critical jet structuring frequency, as given by the nozzle Strouhal number, $S_{d}$, but the exact resonance is dependent on the contractions at each end of the organ-pipe. For instance, if both $\left(D_{s} / D\right)^{2}$ and $(D / d)^{2}$ are large, then the first mode resonance in the pipe will occur when the sound wave length in the fluid is approximately four times $I_{p}$ : Acoustic analyses and experimentation have led to the following approximation, useful for estimating the length of the organ-pipe:

$$
\frac{L_{p}}{d} \simeq \frac{K_{n}}{M S_{d^{*}}}
$$

where the "mode parameter", $K_{n}$ is given by:

$$
\begin{aligned}
K_{n}=\text { func. }\left\{n,\left(\frac{D_{S}}{D}\right)^{2},\left(\frac{D}{d}\right)^{2}\right\} & =\frac{2 n-1}{4} \text { for }\left(\frac{D_{s}}{D}\right)^{2} \text { and }\left(\frac{D}{d}\right)^{2} \gg 1 \\
& =\frac{n}{2} \operatorname{for}\left(\frac{D_{s}}{D}\right)^{2} \gg 1, \text { but }\left(\frac{D}{d}\right)<4
\end{aligned}
$$

In these expressions:

$n=$ mode number of the organ-pipe,

$S_{d^{*}}=$ critical Strouhal number, $f d / v \simeq 0.3$

$M=$ Mach number, $V / c$.

other examples of ORGAN-PIPE CAVIJET concepts are shown in FIg. 11, where single diameter, "two-step", and "three-step" diameter versions are indicated.

When the contractions are as designated in Eq. [9b], then an empirical relation found useful for designing an ORGAN-PIPE CAVIJET is:

$$
M=\frac{n}{2 S_{d}^{*}}\left[\frac{d}{L_{p}}-0.86\left(\frac{d}{L_{p}}\right)^{2}\right]
$$

In Fig. 12, comparisons are shown between the curves given by Eq. [10] and experImental measurements of jet structuring conducted in the air facility described in Sect. 3.1. It can be seen that the experimental data tend to fall within a band of Strouhal numbers, at each mode, of about: $0.4 \leq S_{d}{ }^{*} 0.5$.

\section{SOME EXPERIMENTAL RESULTS}

\subsection{Cavitation Incept1on}


As discussed in Sect. 1.1, one of the motivations for this study of self-resonating cavitating jets was to determine whether new nozzles could be developed whlch would exhibit larger cavitation numbers at inception, $\sigma_{1}$. Comparisons of the inception for thiree nonresonating nozzle types are shown in Fig. 13, where the trends of $\sigma_{1}$ versus Reynolds number are plotted. It should be emphasized that this figure is intended to indicate only general trends; $\sigma_{1}$ observations are highly dependent on detalls in nozzle design and fabrication, boundary layer characteristics, nuclei distributions and gas content in the fluid. For this reason, we prefer to plot (as in Fig. 14) relative curves of $\sigma_{1} / \sigma_{1,0}$, where $\sigma_{i, 0}$ represents a base-line measurement with a standard nozzle under the same test conditions.

The curves in Fig. 14 summarize the results of cavitation inception measurements for each of the three self-resonating concepts. A curve for a conventional, centerbody CAVIJET is also given for comparison. Note that the downward trend of $\sigma_{1}$ seen with respect to Reynolds number for the ORGAN-PIPE CAVIJET is accentuated by the way these data are normalized. That is, because the trend for $\sigma_{i, o}$ (inception for the plain CAVIJET nozzle, see Fig. 13) is steeply upward with increasing Reynolds number, dividing by these $\sigma_{1,0}$ values tends to create an exaggerated downward tendency for $\sigma_{1} / \sigma_{1,0}$

The inception curve for the PULSER-FED CAVIJET nozzle has a well-defined peak within the range of Reynolds numbers in Fig. 14. The resonance in this nozzle shows the strong effect which jet structuring can have on inception of cavitation. Comparable peaks for the ORGAN-PIPE and PULSER nozzles did not occur in the range of Reynolds numbers (or Mach numbers) shown here. However, such peaks in $\sigma_{1}$, associated with critical resonant frequencies, have been observed for each type of self-resonating cavitating jet which has been examined. As seen in Fig. 14, each of the three types of self-resonating concepts have substantially higher inception numbers for cavitation than either the plain or centerbody versions of a "conventional" cavitating jet.

\subsection{Rock Cutting}

A typical comparison is shown in Fig. 15 between a self-resonating cavitating nozzle and a conventional ("SMITH") nozzle* supplied for use in a roller-cone deep-hole drill bit. Seen is a top view of one face of a $15.2 \mathrm{~cm}(6 \mathrm{in.})$ cube specimen of Indiana limestone. To allow a half-circle cut to be made by each nozzle, one half of the rock face was protected by a metal plate. The single pass was then made at a translation velocity of $6.4 \mathrm{~mm} / \mathrm{s}$ - starting and stopping the rotation while the jet was impinging on the plate. The nozzle was then changed, the plate shifted to protect the other half of the cube-face, and the same conditions were run again. In this way, variations due to rock properties could be minimized. Under these conditions, the mean slot depth cut by the ORGAN-PIPE CAVIJET was 4.3 times deeper than that cut by the SMITH nozzle.

Similar slot-cutting comparisons are shown in Table 1 at several cavitation numbers. Note that, for the single pass tests, as $\sigma$ is increased the performance of the ORGAN-PIPE CAVIJET improves relative to the SMITH nozzle. The cavitation inception number for this CAVIJET nozzle was more than twice that for the SMITH nozzle. This result is consistently observed, that is, the jet having the higher inception cavitation number is found to be more erosive than a jet of the same size, operated under identical conditions, but having a lower $\sigma_{1}$.

\subsection{Underwater Cleaning}

In a field trial of an early PULSER CAVIJET design, cleaning rates more than twice those achieved by a conventional CAVIJET of the same size were reported (24). This trial occurred during underwater cleaning of the "keel block areas" on the hull of an aircraft carrier, the USS LEXINGTON (CVT-16) which had just come out of drydock. The hull had been cleaned and repainted in drydock, except for those areas which had rested on the keel blocks. Navy divers, using underwater CAVIJET tools (25) developed by SEACO and HYDRONAUTICS, then proceeded to remove the old paint and corrosion products from the keel block areas while the LEXINGTON floated next to the dock in Pensacola, Florida. These divers reported, albelt subjectively since precise time trials were

* Manufactured by SMITH TOOL, Division of Smith International, Inc., Irvine, California, U.S.A. 
not taken, that a PULSER nozzle, with $d=2.5 \mathrm{~mm}(0.10 \mathrm{In}$ ) (Bee F1g. 8a) prepared the required white metal steel surface sultable for underwater repainting at a rate more than twice that of a conventional CAVIJET aozzle with the same orifice diameter. ThIs trial involved 11mited use of the PULSER - most of the cleaning was with the conventional CAVIJET since only the latter nozzle has recelved an officlal Approval for Navy Use (ANU). We will be seeking an ANU for one or more of the self-resonating cavitating jet nozzles after further evaluation to determine the best designs for this underwater cleaning application.

\section{CONCLUDING REMARRS}

This paper has summarized some of the early results from an ongoing effort to develop new cavitatirg jets wh1ch might perform more effectively, particularly at high cavitation numbers (1.e., under deep submergence). Additional details can be found in the report, recently released by Sandia National Laboratories, which describes the feasibility study phase of this program (26). From this study, the following conclusions have been drawn:

a. Higher inception cavitation numbers can be achieved in a fet which is structured into discrete ring vortices at preferred resonant frequencies.

b. Several concepts have been demonstrated to be capable of producing a passive self-oscillation in a submerged cavitating fet. Three of these self-resonating nozzle types, the PULSER, PULSER-FED, and ORGAN-PIPE CAVIJET designs, have been found to have higher inceptions for cavitation than conventional (nonresonating) CAVIJETS and typical drill bit nozzles.

c. Limited rock cutting and cleaning trials have demonstrated a correlation between higher cavitation inception numbers and greater erosivity in submerged cavitating jets.

\section{ACKNOWLEDGEMENTS}

This study has been supported by the U.S. Department of Energy, Division of Geothermal Energy, under Sandia Laboratories Contract Nos, 13-5111 and 13-5129, with cost-sharing by NL/Hycalog, Houston, Texas, U.S.A.

\section{REFERENCES}

1. Johnson, V. E., Jr., et al.: "Tunnelling, fracturing, drilling and mining with high speed water jets utilizing cavitation damage". Proc. First Int'1. Sympos. on Jet Cutting Technology (Coventry, U.K.: April 5-7, 1972) Cranfield, U.K., BHRA Fluid Engineering, 1972, Paper A3, 19 PP.

2. Conn, A. F. and Johnson, V. E., Jr.: "Further applications of the CAVIJET" (cavitating water jet) method". Proc. Second Int'1. Sympos. on Jet Cutting Technology (Cambridge, U.K.: April 2-4, 1974) Cranfield, U.K., BHRA Fluld Engineering, 1974, Paper D2, 14 Pp.

3. Conn, A. F. and Rudy, S. L.: "Cutting coal with the CAVIJET" cavitating water jet method". Proc. Third Int'l sympos, on Jet Cutting Technology (Chtcago, v.S.A.: May 11-13, 1976) Cranfield, U.K., BHRA Fluid Engineering, 1976, Paper D8, 9 pp.

4. Conn, A. F., Rudy, S. L., and Mehta, G. D.: "Development of a CAVIJET" system for removing marine fouling and rust". Proc. Third Int'1 Sympos. on Jet Cutting Technology (Chicago, U.S.A.: May 11-13, 1976) Cranfield, U.K., BHRA Fluid Engineering, 1976, Paper G4, 14 Pp.

5. Conn, A. F. and Rudy, S. L.: "Conservation and extraction of energy w1th the CAVIJET" Proc. Fourth Int'1. Sympos. on Jet Cutting Technology (Canterbury, U.K.: April 12-14, 1978) Cranfield, U.K., BHRA Fluid Engineering, 1978, Vol. 1, Paper H2, 20 PP.

6. Conn, A. F. and Johnson, V. E. Jr.: "The fluid dynamics of submerged cavitating jet cutting". Proc. Fifth Int'1 Sympos. on Jet Cutting Technology (Hanover, F.G.R.: June 2-4, 1980), Cranfield, U.K., BHRA Fluid Engineering, 1980, Paper Al. Pp. 1-14.

7. Maurer, W. C.: "The 'perfect cleaning' theory of rotary drilling". Journal of Petroleum Technology, Vol. 14, 1962, Pp. 97-101. 
8. Crow, S. C. and Champagne, F. H.: "Orderly structure in fet turbulence". Journal of Fluid Mechanics, Vol. 48, Part 3, August 1971, pp. 547-591.

9. Moore, C. J.: "The role of shear layer Instability waves in jet exhaust noise". Journal of Fluid Mechanics, Vol. 80, 1977, pp. 321-367.

10. Kibens, V.: "Discrete noise spectrum generated by an acoustically.excited jet". Paper 79-0592, AIAA Fifth Aeroacoustics Conference, March 12-14, 1979.

11. Morel, T.: "Dxperimental study of a jet driven Helmholtz oscillator". ASME, Journal of Fluids Engineering, Vo1. 101, September 1979, PP. 383-390.

12. Wylie, E. B.: "Pipeline dynamics and the pulsed jet". Proc., First Int' 1 Sympos. on Jet Cutting Technology (Coventry, U.K.: April 5-7, 1972) Cranfield, U.X., BHRA Fluid Englneering, 1972, Paper A5, 12 Pp.

13. Streeter, V. L. and Wylle, E. B.: Hydraulic Transients, McGraw H111, 1967.

14. Nebeker, E. B. and Rodriguez, S. E.: "Parcussive water fets for rock cutting". Proc. Third Int '1. Sympos. on Jet Cutting Technology (Chicago,-U.S.A.: May 11-13, 1976) Cranfield, U.K., BHRA Fluid Engineering, 1976, Paper Bl, 9 Pp.

15. Llchtarowicz, A. and Nwachukwu, G.: "Erosion by an interrupted jet". Proc. Fourth Int'1. Sympos. on Jet Cutting Technology (Canterbury, U.K.: April 12-14, 1978) Cranfield, U.K., BHRA Fluid Engineering, 1978, Vol. 1, Paper B2, 6 pp.

16. Erdmann-Jesnitzer, F., Louis, H., and Schikorr, W.: "Cleaning, drilling and cutting by interrupted jets". Proc. Fifth. Int'l Sympos. on Jet Cutting Technology (Hanover, F.G.R.: June 2-4, 1980) Cranfield, U.K., BHRA Fluid Engineering, 1980, Paper B1, PP. 45-55.

17. Galle, E. M. and Woods, H. B.: United States Patent Number 3,405,770, 0ctober 15 , 1968.

18. Fülöp, M. and Rókár, G.: United States Patent Number 4,071,097, January 31, 1978.

19. Sondhauss, C.: Annual Physics, Volume 97, pg. 1854.

20. Helmholtz, Philosophical Magazine, Volume 36, 1868, pg. 337.

21. Rayleigh, J.W.S.: Theory of Sound, Chapter XXI, 2nd Edition, MacMillan (Repub1ished 1945, Dover Publications), Pp. $1825 \mathrm{ff}$.

22. Rockwell, D. and Naudascher, E.: "Self sustained oscillations of impinging free shear layers". Annual Review of Fluid Mechanics, Volume 11, 1979.

23. Milne-Thomson, L. M.: Theoretical Hydrodynamics, MacMillan, New York, 1969, PP. 351-354.

24. May, Robert A., SEACO, Incorporated, CAVICO Division, private communication, September, 1981 .

25. Conn, A. F., Johnson, V. E., Jr., Lindenmuth, W. T., and Frederick, G. S.: "Some industrial applications of CAVIJET cavitating fluid jets". Proc. 1st U.S. Water Jet Sympos., Golden, Colorado, April 1981.

26. Johnson, V. E., Jr., Lindenmuth, W. T., Conn, A. F., and Frederick, G. F.: "Feasibility study of tuned-resonator, pulsating cavitating water jet for deephole drilling". Sandia Labcratories Contractor Report SAND81-7126, August 1981 (originally: HYDRONAUTICS, Incorporated Technical Report 8001-1, May 1981), 142 PP. 
Table 1 - Slot Cutting Comparisons

NOZZLES: Conventional SMITH, $d=4.3 \mathrm{~mm}(0.170 \mathrm{1n}$.

ORGAN-PIPE CAVIJET, $d=4.7 \mathrm{~m}(0.185 \mathrm{in}$.

ROCK: Indiana limestone; STANDOFF: $X / d=3$

TRANSLATION VELOCITY: $6.4 \mathrm{~mm} / \mathrm{s}(0.25 \mathrm{in.} / \mathrm{s})$

\begin{tabular}{|c|c|c|c|}
\hline $\begin{array}{c}\text { Pressure, } \\
\text { Nozzle } \\
\Delta \mathrm{p}, \mathrm{MPa} \text { (psi) }\end{array}$ & $\begin{array}{c}\text { Ambient } \\
\text { Pressure, } \\
\mathrm{P}_{\mathrm{a}}, \operatorname{MPa} \text { (psi) }\end{array}$ & $\begin{array}{c}\text { Cavitation } \\
\text { Number, } \\
\sigma\end{array}$ & $\begin{array}{l}\text { Mean Slot } \\
\text { Depth Ratio, } \\
\text { CAVIJET } \\
\text { SMITH }\end{array}$ \\
\hline \multicolumn{4}{|c|}{ SINGLE PASS } \\
\hline $17.4(2,530)$ & $1.2 \quad(170)$ & 0.067 & -1.16 \\
\hline $17.2(2,500)$ & $4.1 \quad(600)$ & 0.24 & 2.54 \\
\hline $17.2(2,500)$ & $8.3(1.200)$ & 0.48 & 2.33 \\
\hline \multicolumn{4}{|c|}{ TWENTY-FIVE PASSES } \\
\hline $8.4(1,225)$ & $2.2 \quad(325)$ & 0.27 & 1.90 \\
\hline
\end{tabular}




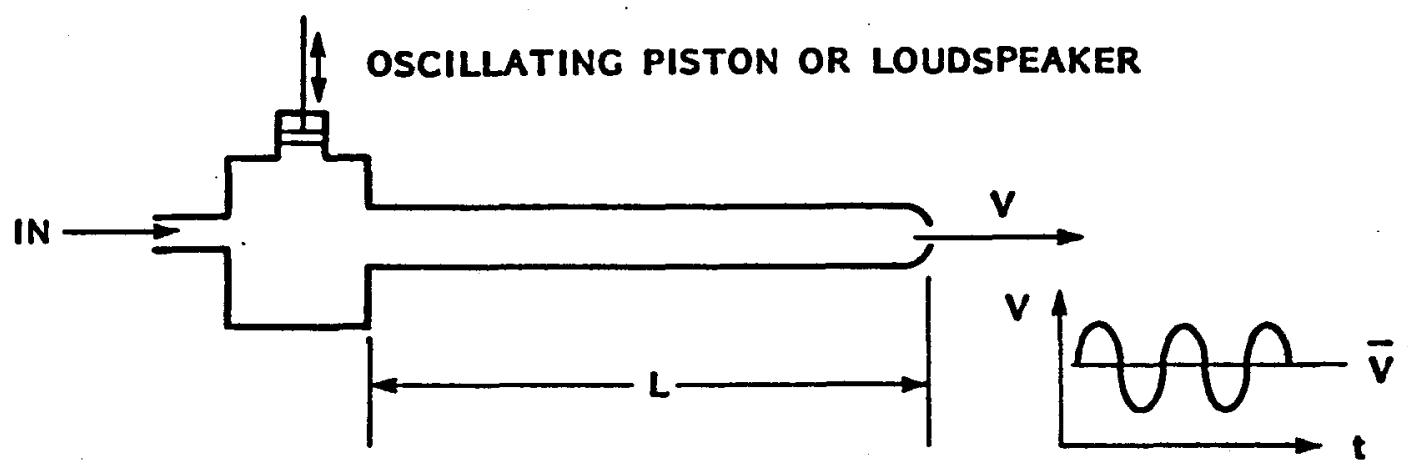

a. OSCILLATING PISTON

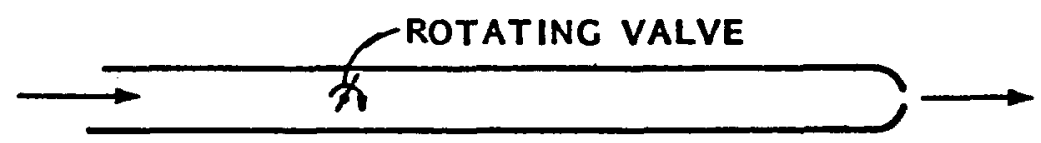

b. OSCILLATING MECHANICAL VALVE

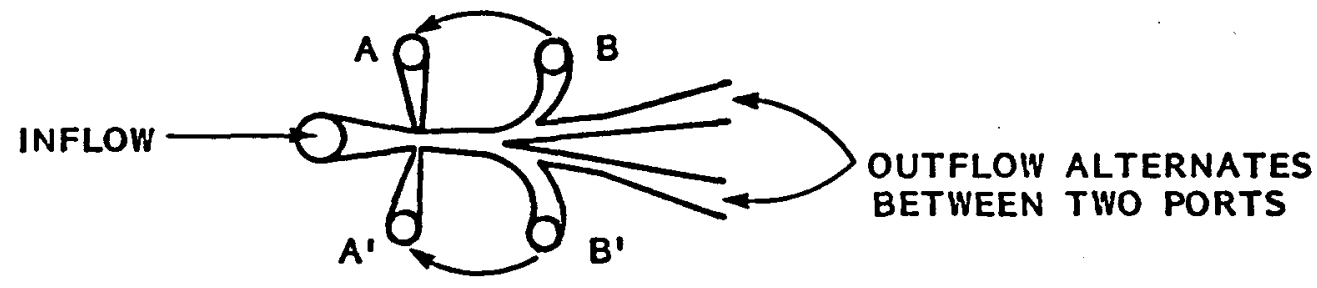

c. FLUID OSCILLATOR VALVE

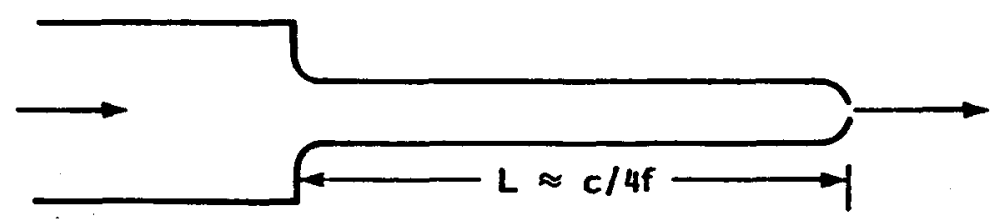

d. ORGAN-PIPE ACOUSTIC OSCILLATOR

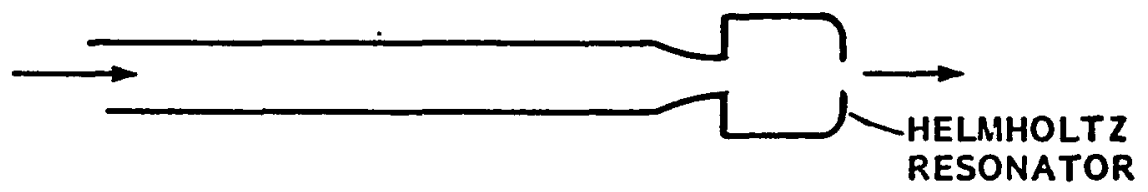

e. HELMHOLTZ ACOUSTIC OSCILLATOR

FIGURE 1 - GENERAL CONCEPTS FOR PULSINC JETS 


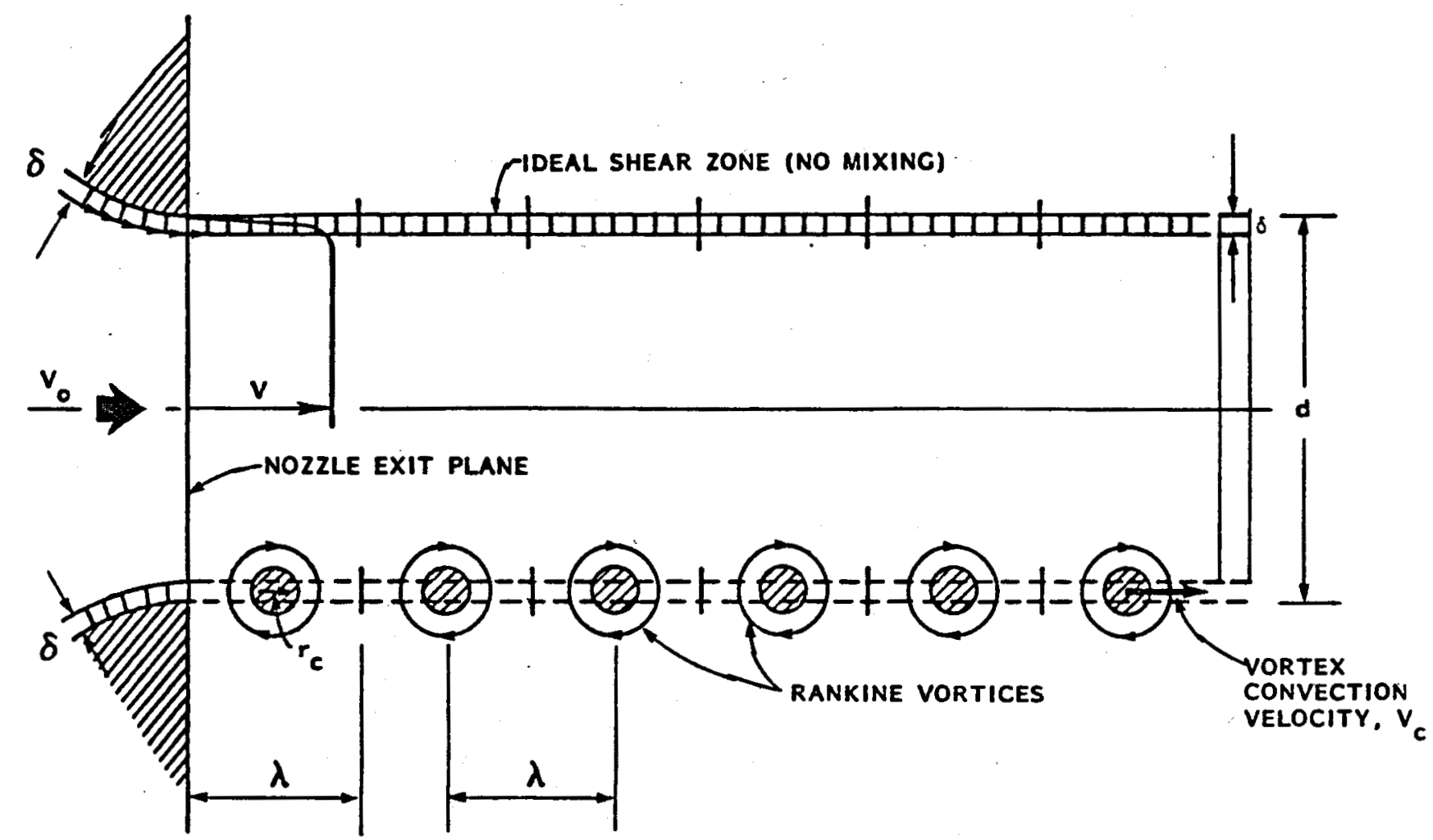

FIGURE 2 - SCHEMATIC OF MECHANISM FOR VORTEX ROLL-UP IN THE SHEAR ZONE OF A SUBMERGED JET 


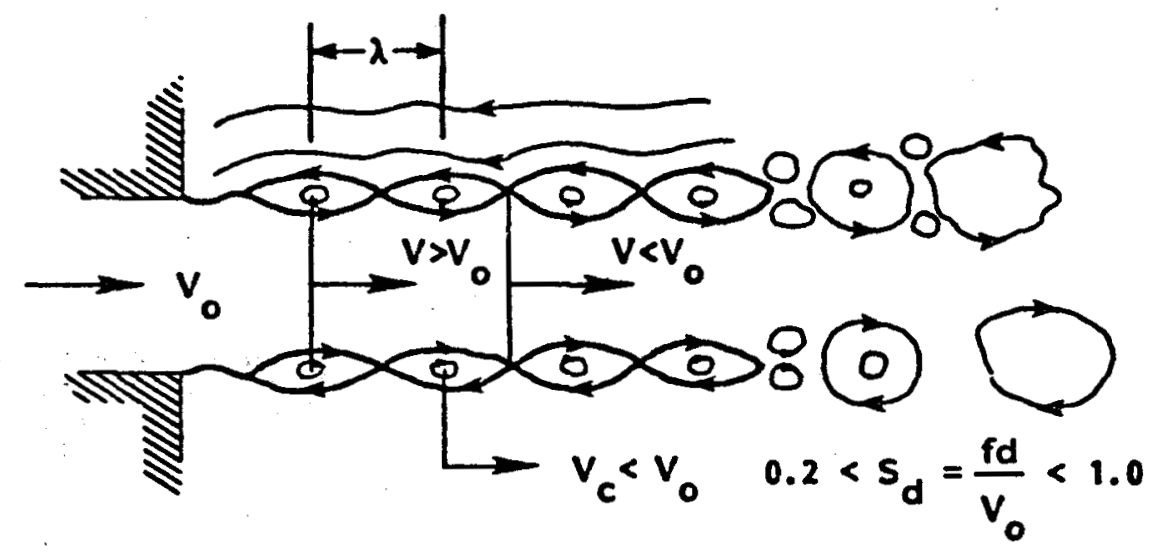

a. STRUCTURED FLOW PATTERN

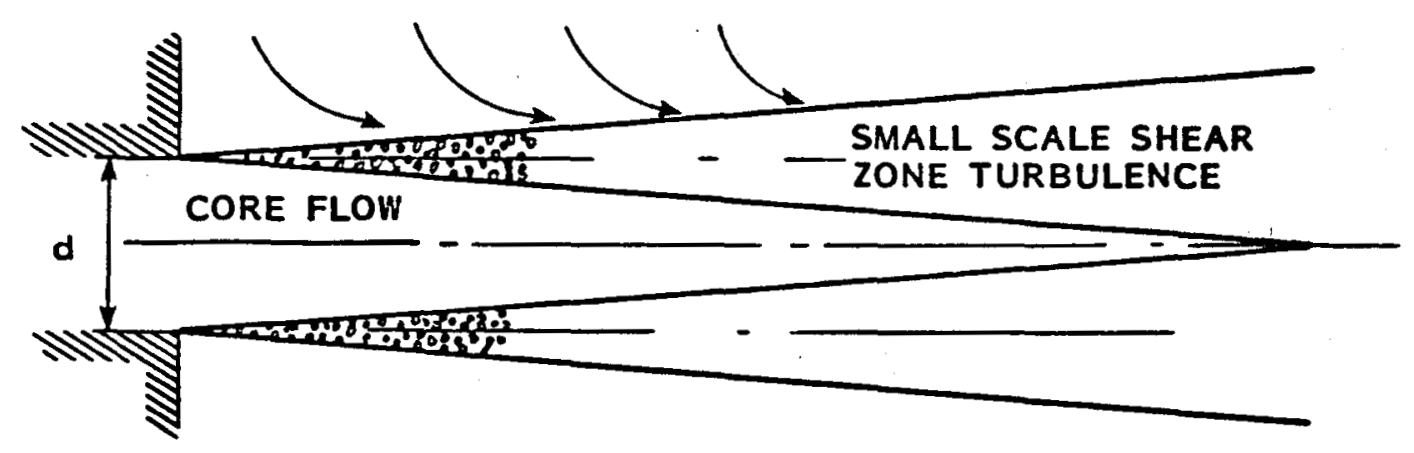

b. UNSTRUCTURED FLOW PATTERN

FIGURE 3 - FREE SUBMERGED JET FLOW PATTERN COMPARISON 


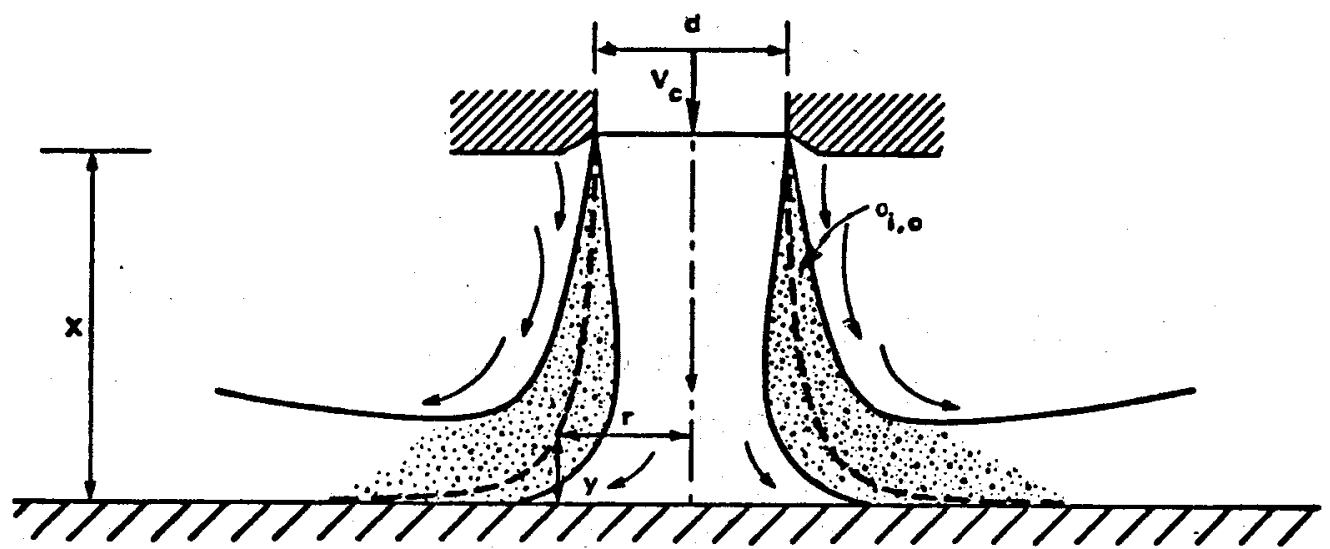

a. UNSTRUCTURED Jet

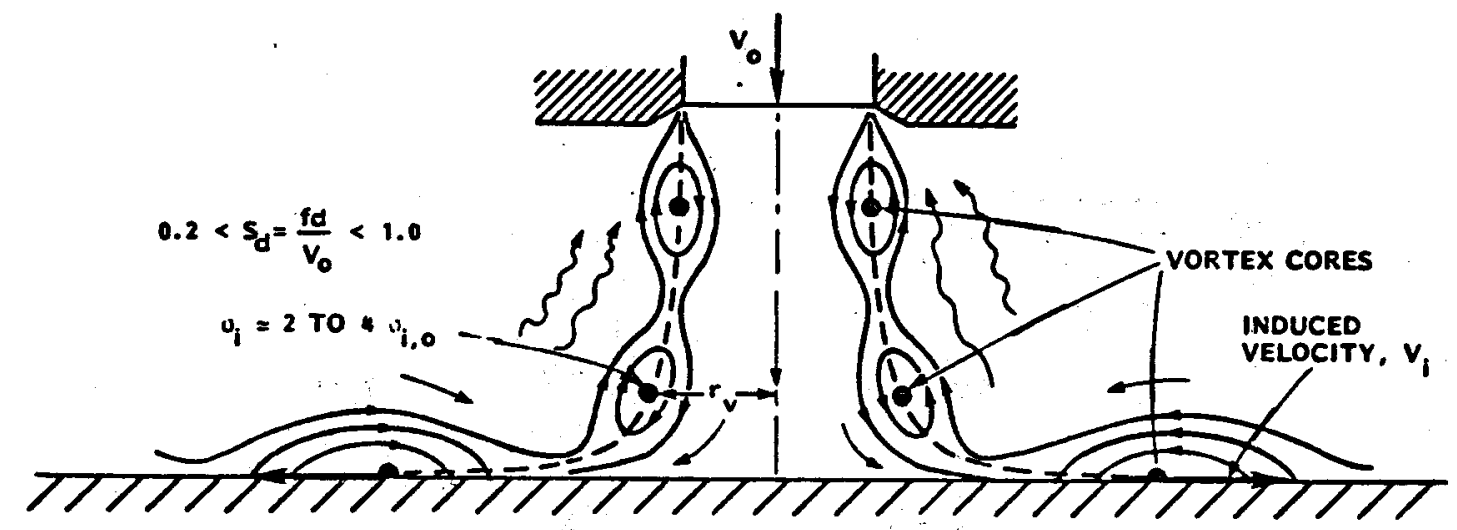

b. STRUCTURED JET

FIGURE - FLOH COMPARISON FOR IMPINGIIC SUBMERCED CAVITATINC JETS

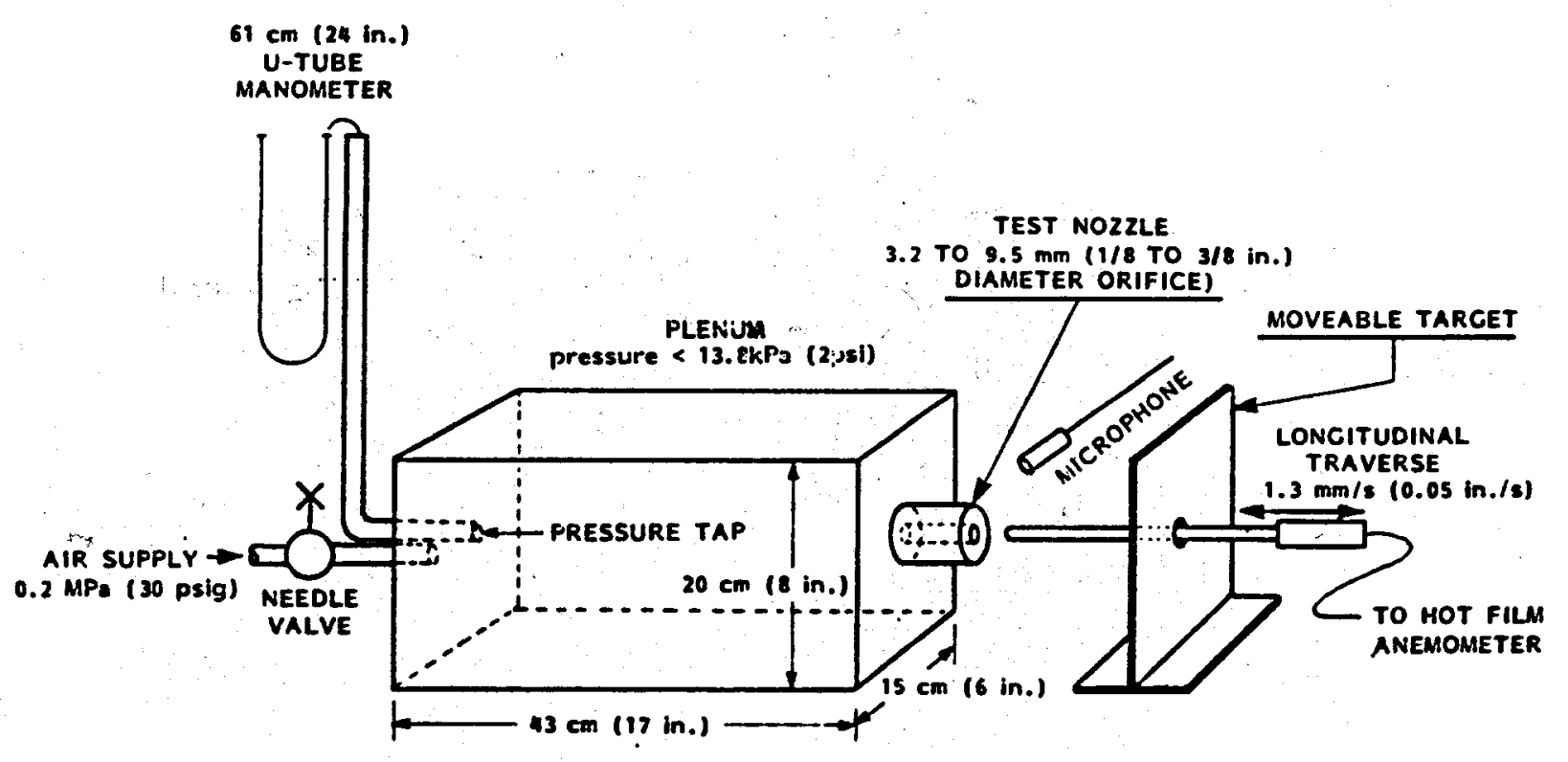

FICURE S - APPARATUS FOR NOZZLE TESTS WITH AIR 

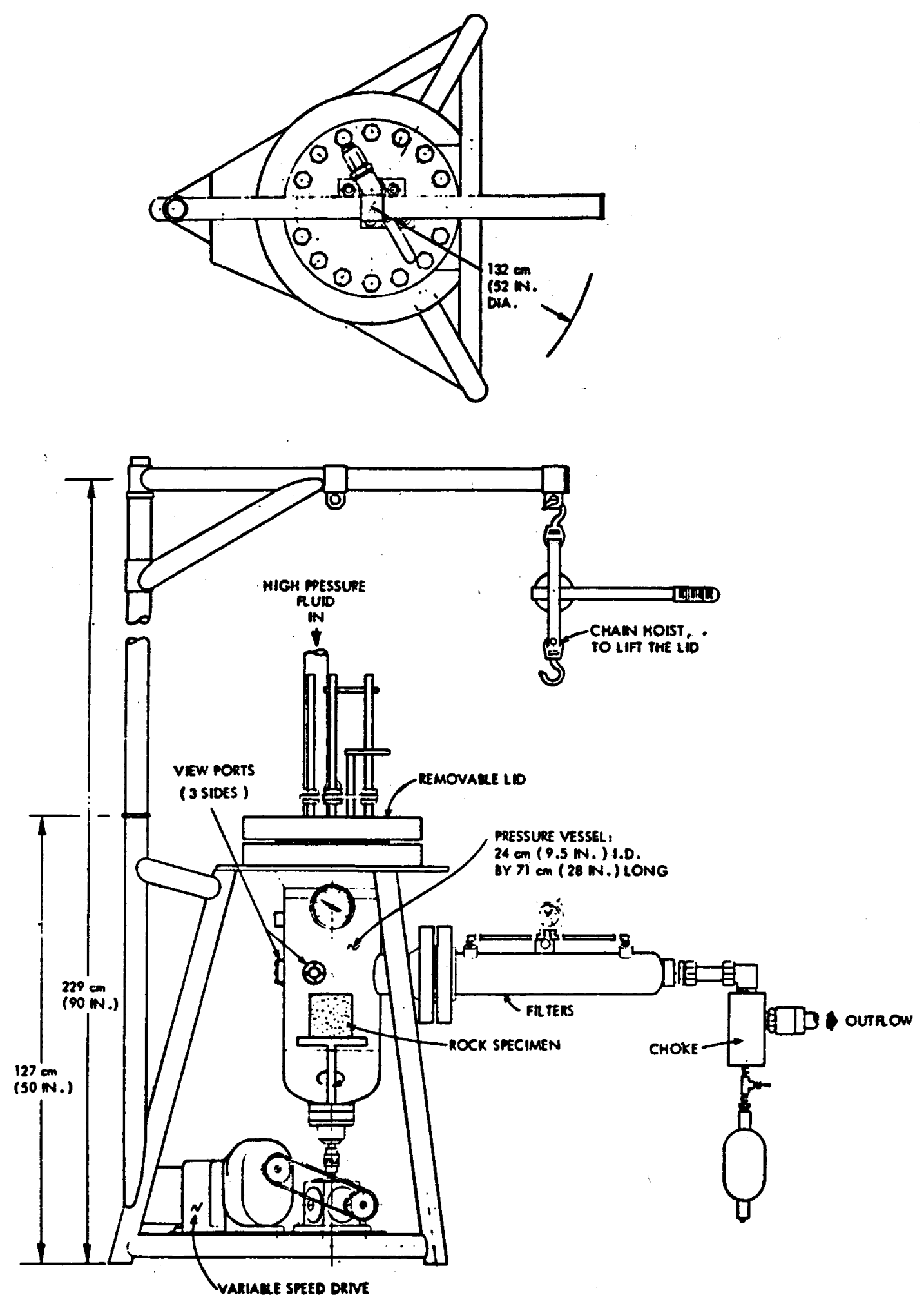

FIGURE 6 - HIGH PRESSURE CELL, HPC 


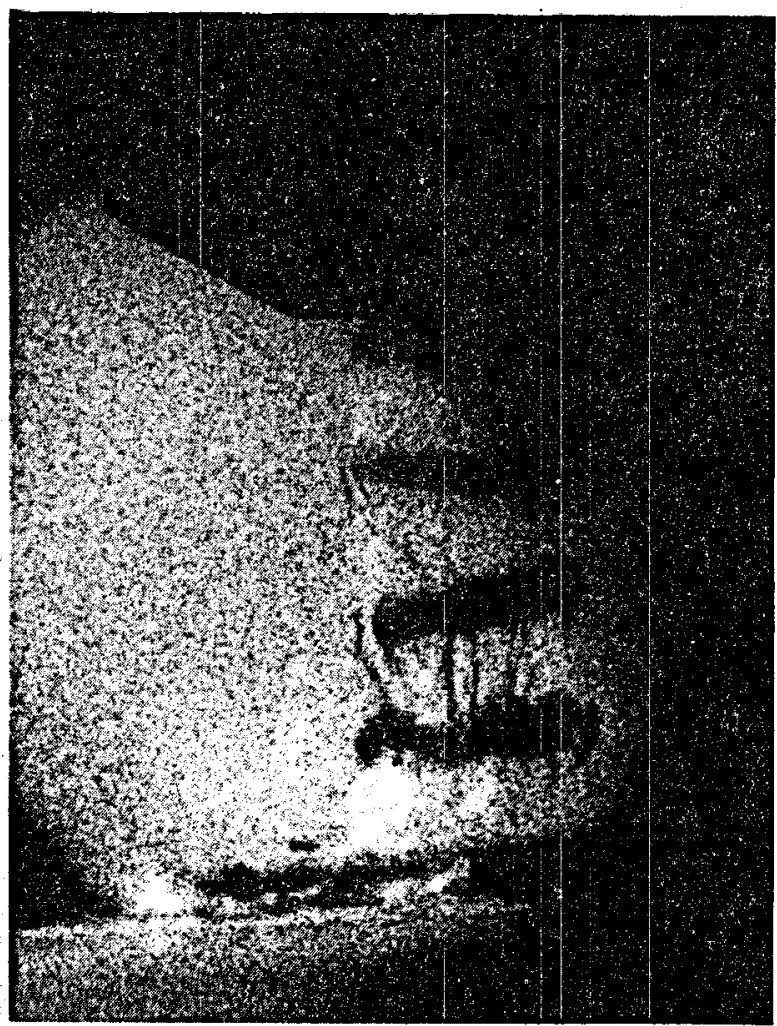

STANDOFF: $X=12.7 \mathrm{~mm}(0.5 \mathrm{in.}) ; X / d=2.7$ NOZZLE PRESSURE DROP: $\Delta p=17.2 \mathrm{MPa}(2,500 \mathrm{psi})$ AMBIENT PRESSURE: $p=3.9 \mathrm{MPa}$ (560 psi) NOZZLE ORIFICE DIAMETER: $d=4.7 \mathrm{~mm}$ (0.186 in.) RESONANCE FREQUENCY: $20.5 \mathrm{kHz}$

STROUHAL NUMBER: $S_{d}=0.52$

CAVITATION NUMBER: $\sigma=0.22$

NOZZLE: ORGAN-PIPE CAVIJET, with two-step pipe configuration. This nozzle is half-scale model of CAVIJET to be used in a threecone roller bit.

FIGURE 7 - TYPICAL PHOTO OF RING VORTICES FROM SELF-RESONATING CAVIJET® NOZZLE. Note vortex spreading across surface of target plate. 


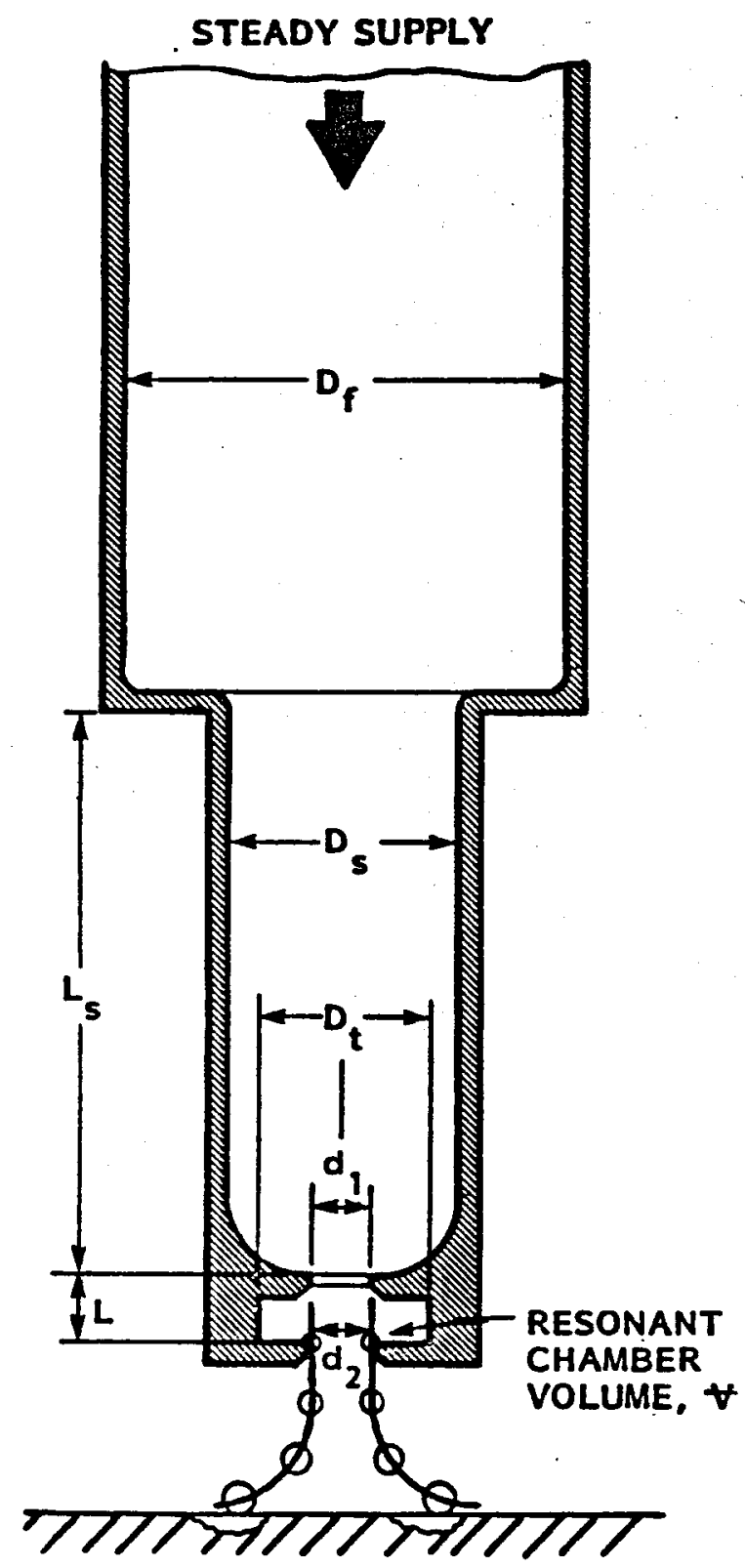

a. BASIC PULSER NOZZLE

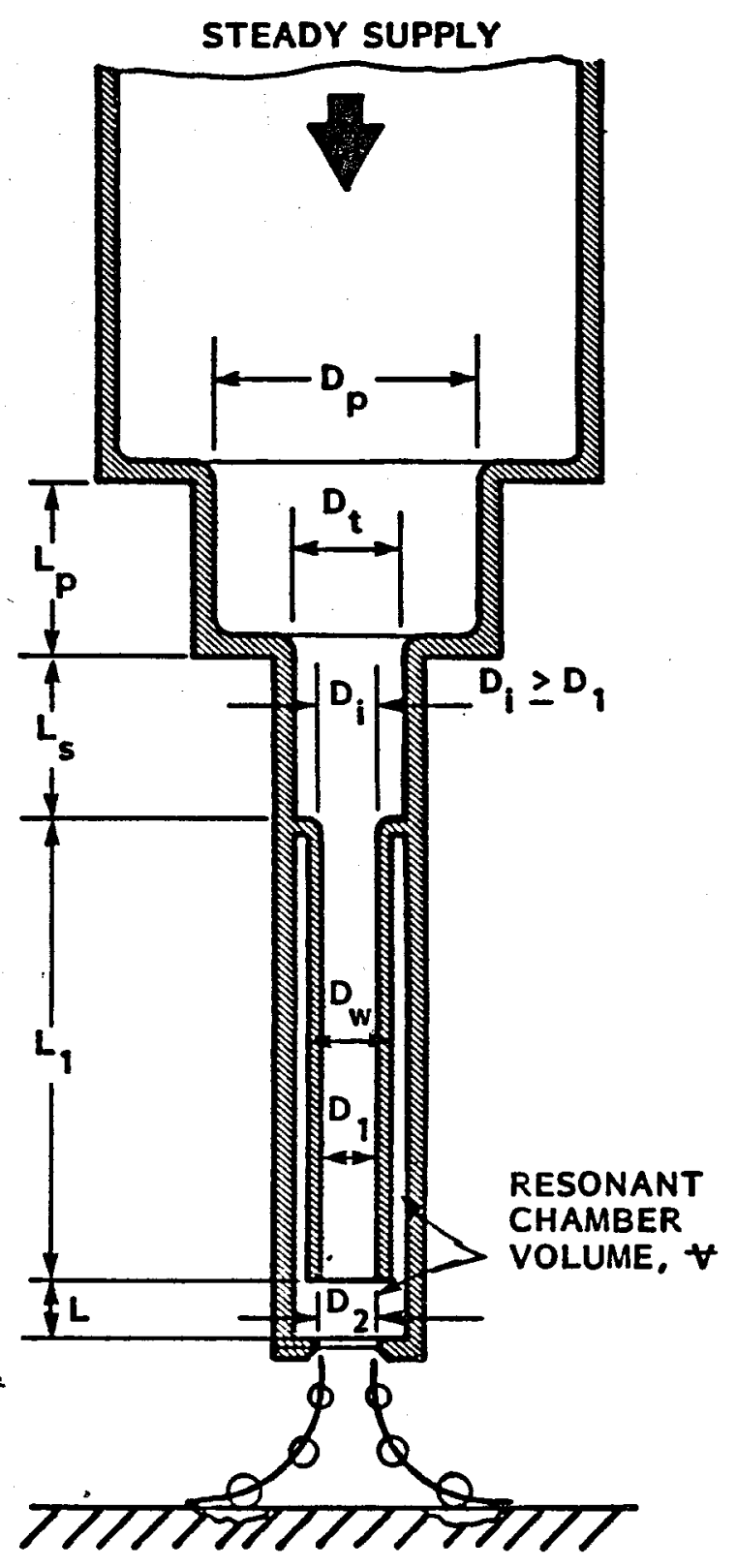

b. LAID-BACK PULSER NOZZLE

FIGURE 8 - CONCEPTS FOR PULSER CAVIJET ${ }^{\circledR}$ NOZZLES 


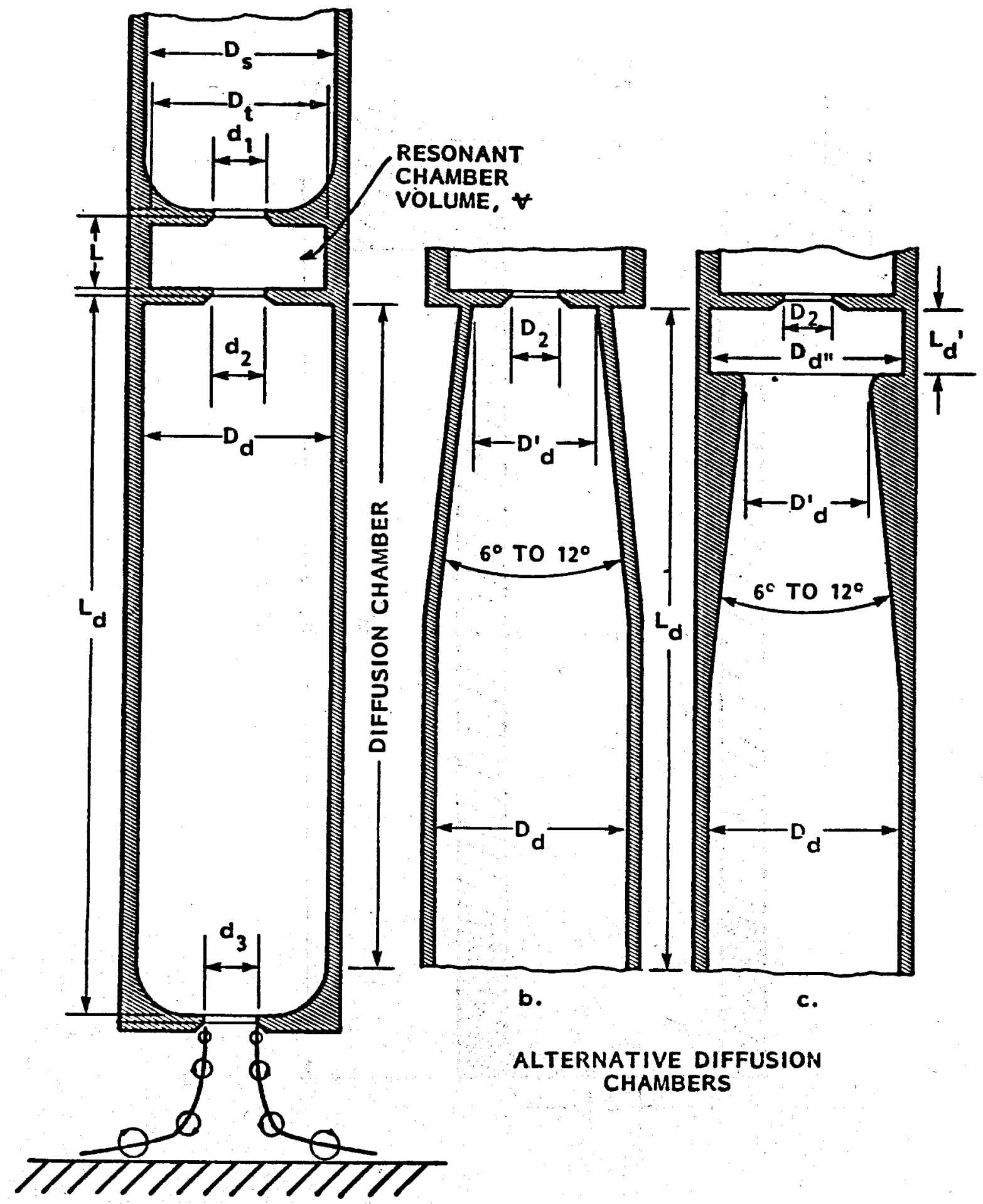

a. BASIC DESICN

FIGURE 9 - CONCEPTS FOR PULSER-FED CAVIJET ${ }^{(1)}$ NOZZLES 


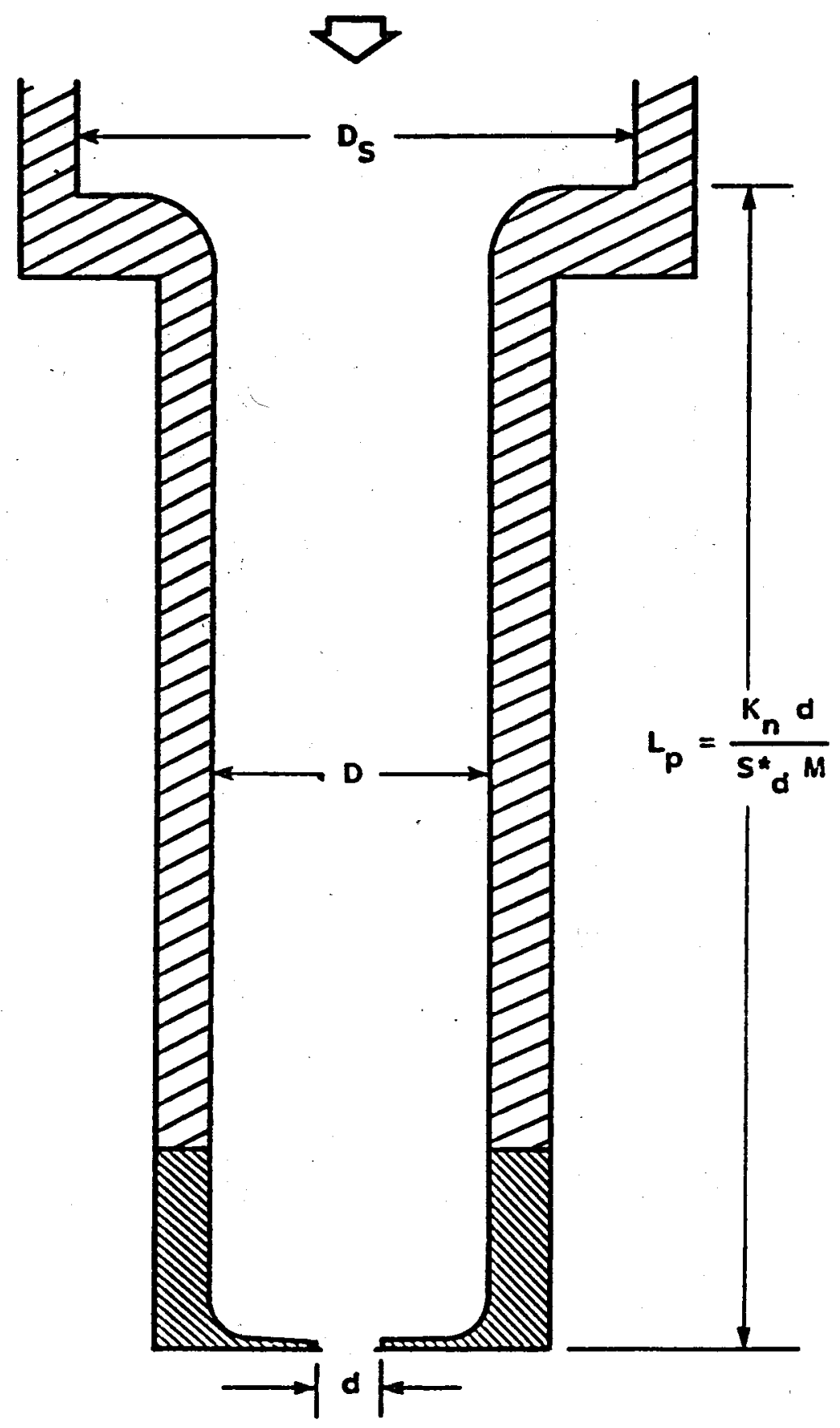

FIGURE 10 - ORGAN PIPE CAVIJET@ NOZZLE CONCEPT

20 

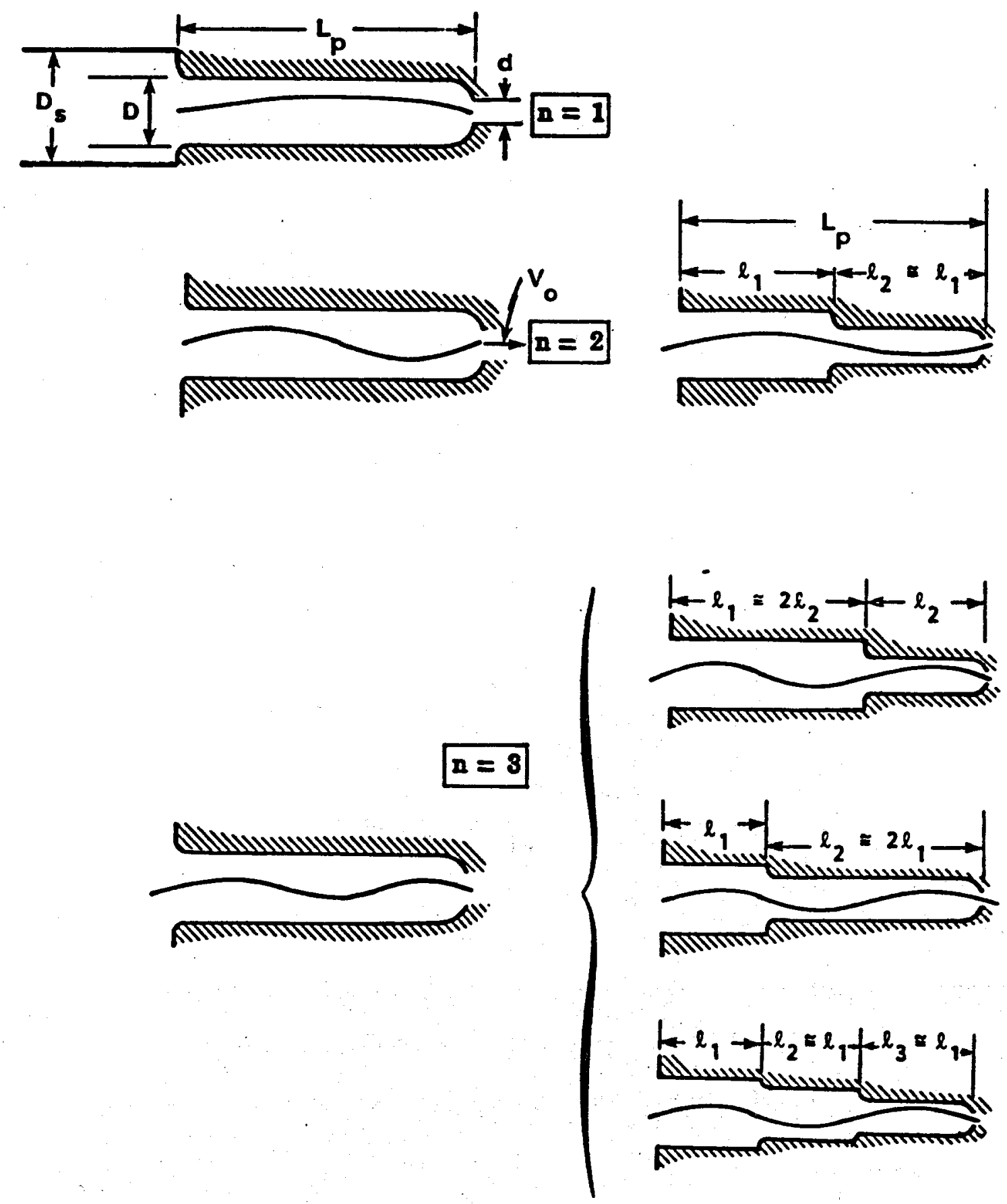

FIGURE 11 - NULTIPLE STEPPED ORGAN-PIPE CAVIJET ${ }^{\circ}$ CONCEPTS 


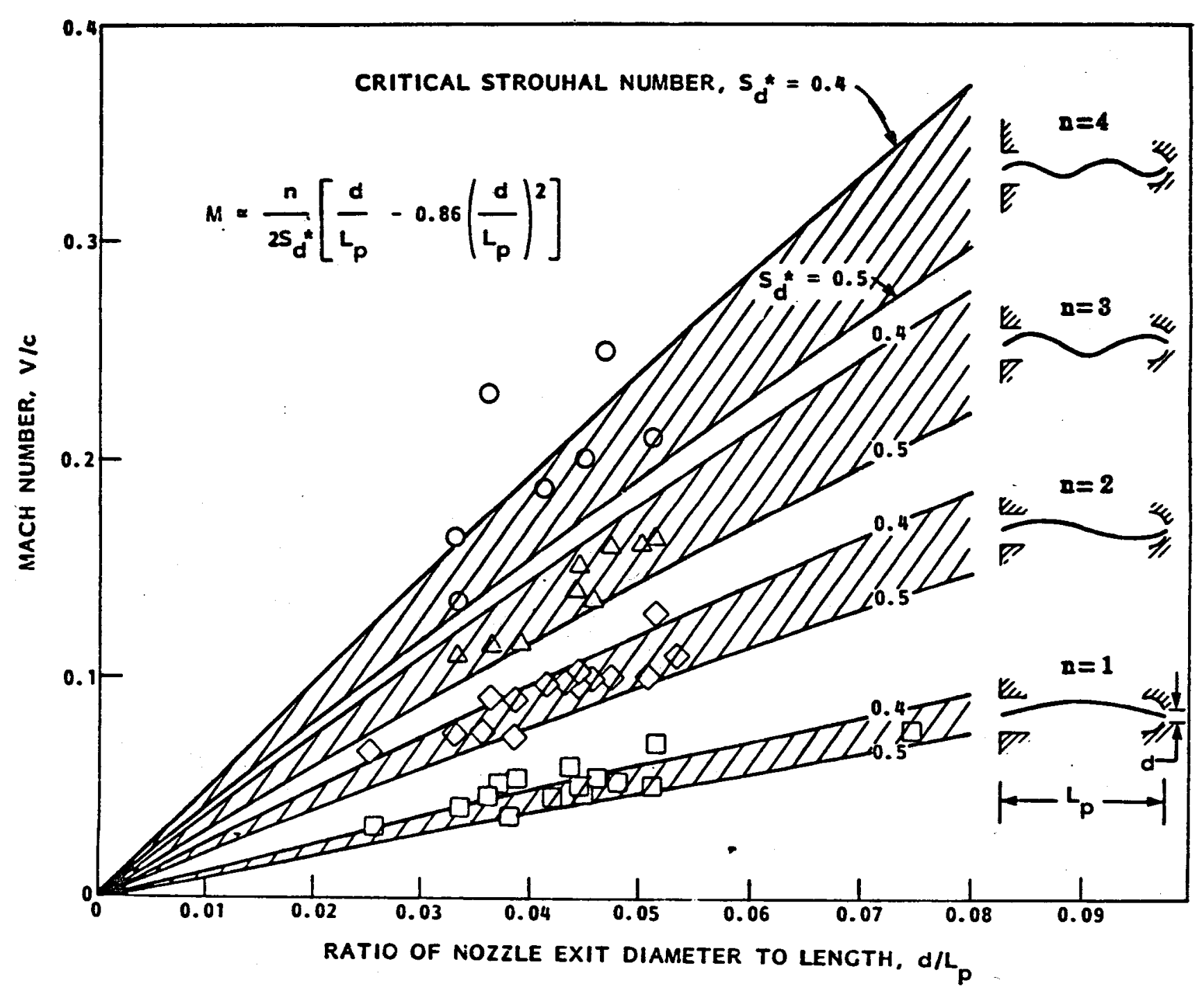
FICURE 12 - RELATION BETWEEN MACH NUMBER AND NOZZLE LENGTH FOR
ORCATI-PIPE CAVIJET NOZZLES 


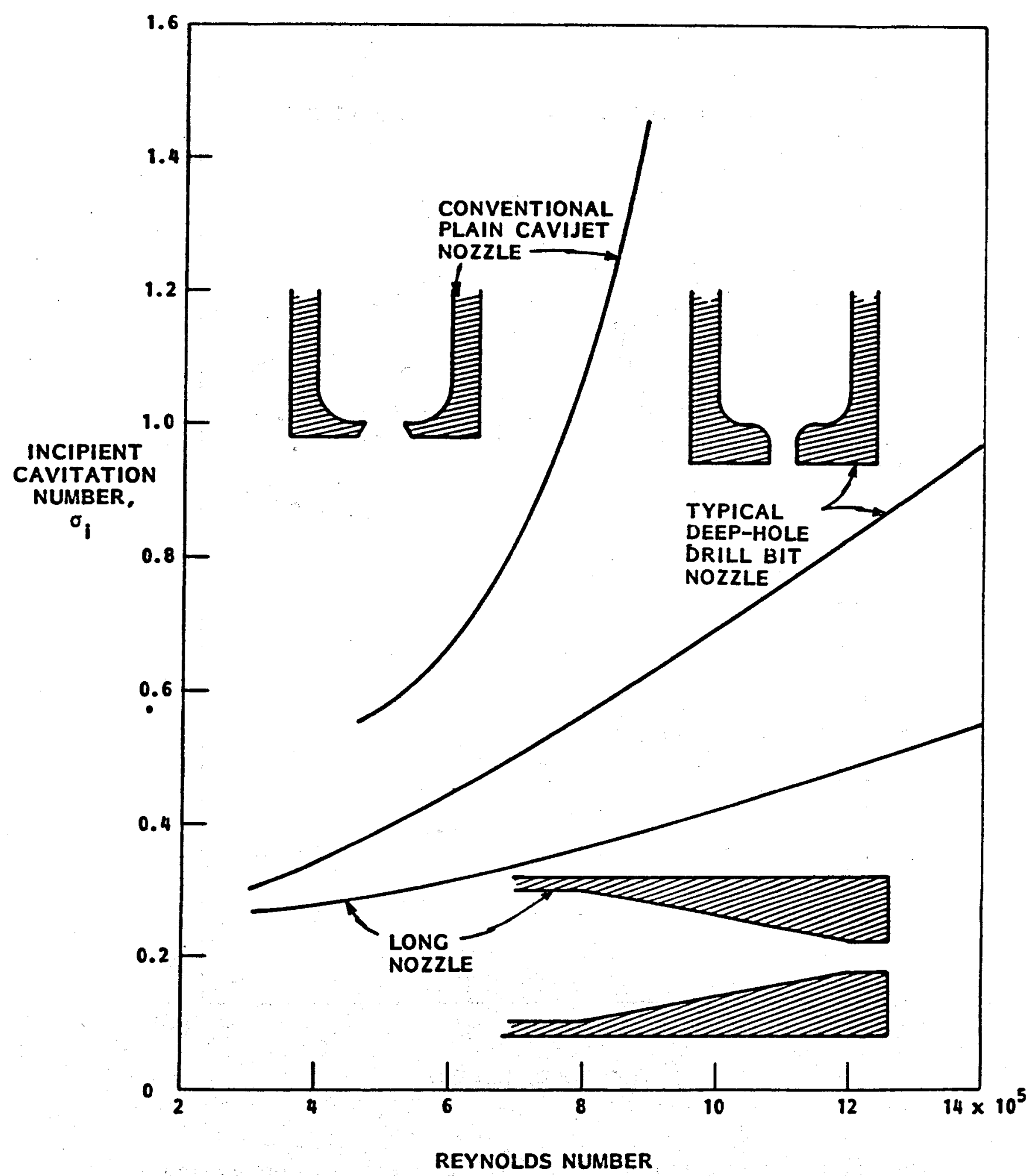

FICURE 13 - APPROXIMATE INCIPIENT CAVITATION NUMIBERS FOR CAVIJETO AND OTHER NOZZLE TYPES (ACOUSTIC DETECTION) 


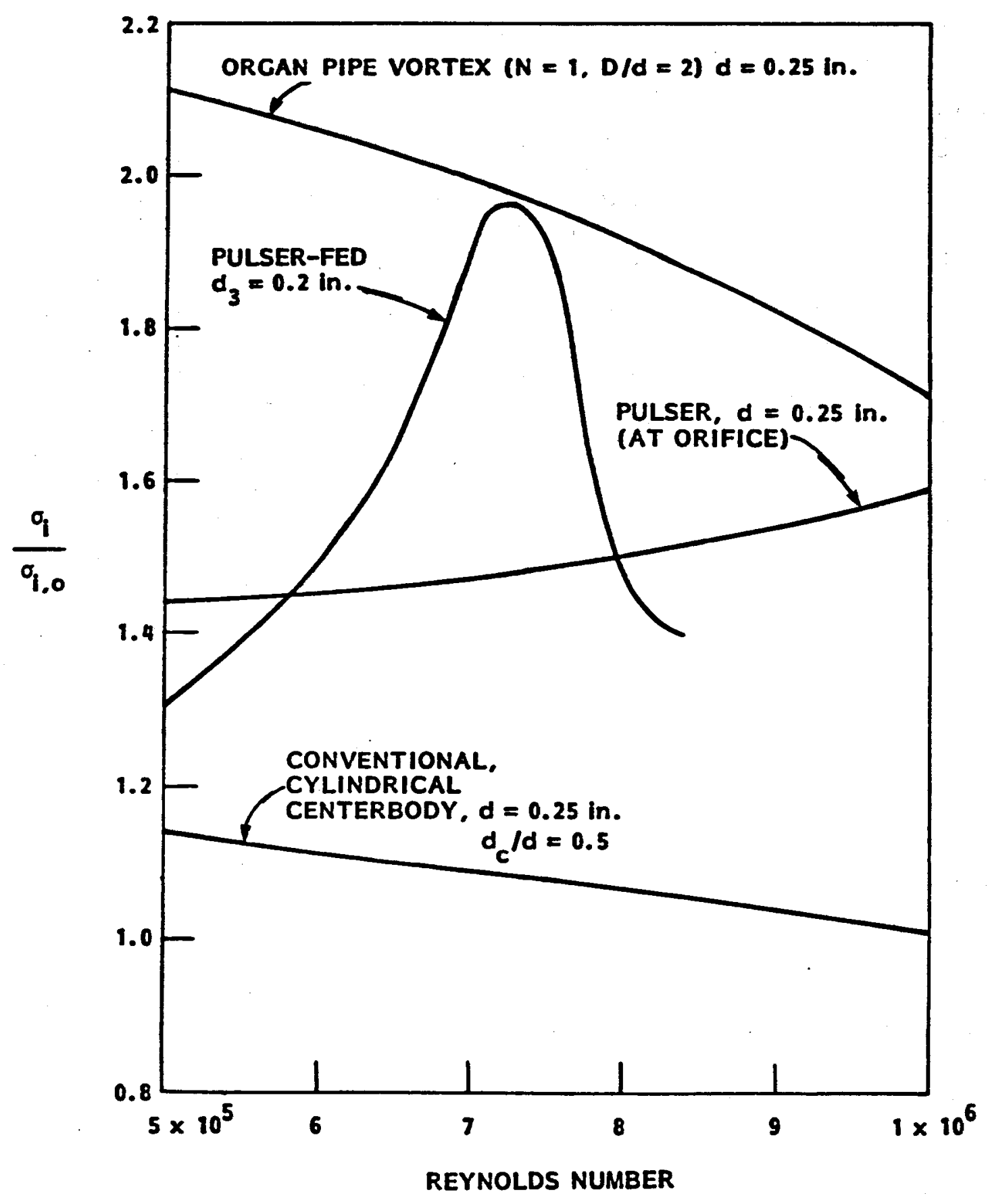

FIGURE 14 - EXPERIMENTAL INCIPIENT CAVITATION NUMBERS FOR VARIOUS NOZZLE TYPES (Relative to conventional

CAVIJETO) $\quad \sigma_{i, 0}=\sigma_{i}$ (plain CAVIJET $\theta^{\text {) }}$ 

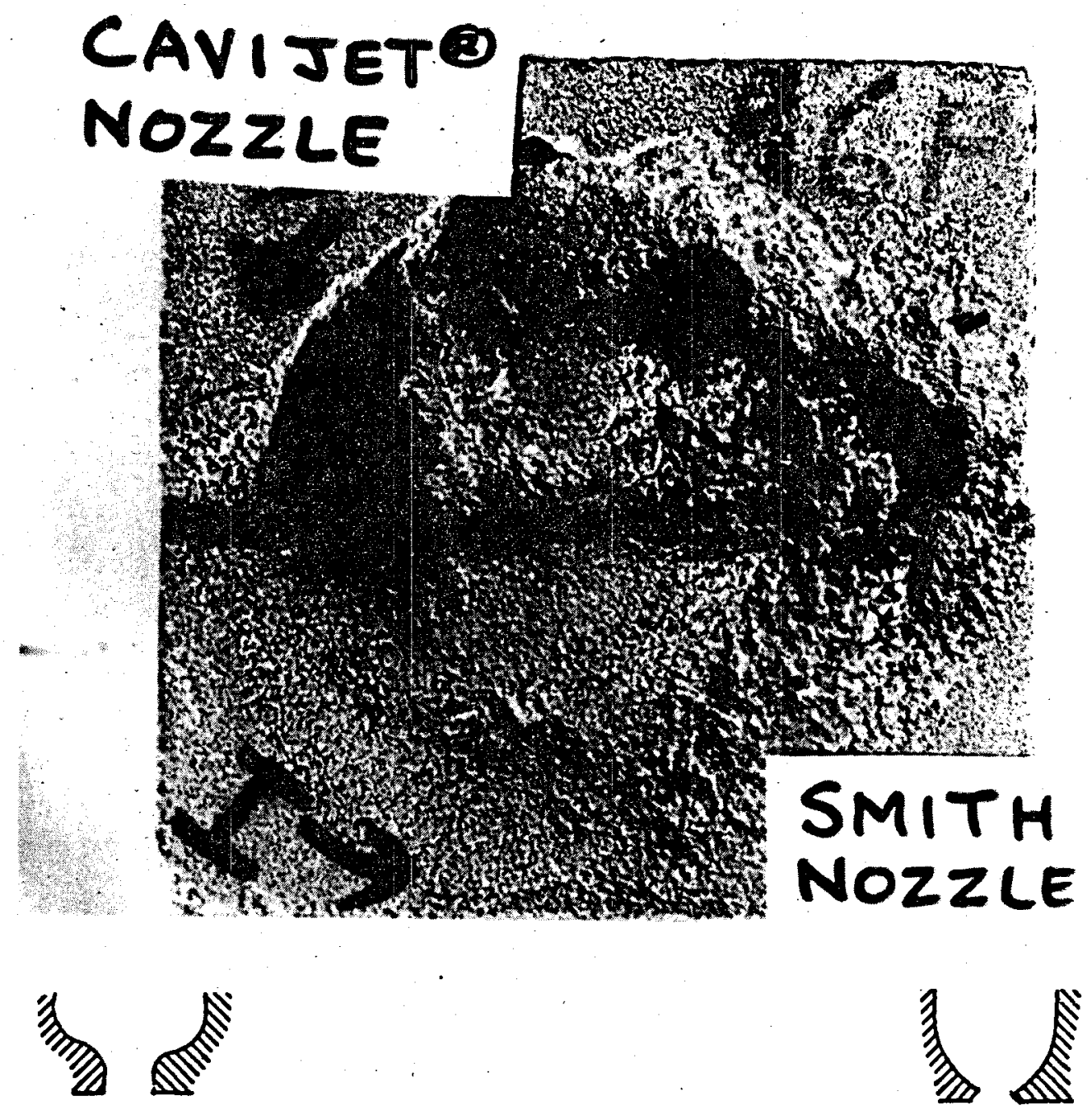

SMITH

ORGAN-PIPE

CAVIJET ${ }^{\circledR}$

ROCK: Indiana limestone, STANDOFF: $x / d=3$

NOZZLE PRESSURE: $\Delta p=10.3 \mathrm{MPa}(1,500 \mathrm{psi})$

AMBIENT PRESSURE: $p_{a}=0.83 \mathrm{MPa}(120 \mathrm{psi})$

CAVITATION NUMBER: $\sigma=0.08$

TEST MODE: Single pass in HPC

TRANSLATION VELOCITY: $v=6.4 \mathrm{~mm} / \mathrm{s}(0.25 \mathrm{in.} / \mathrm{s})$

NOZZLE ORIFICE DIAMETER: $d=6.4 \mathrm{~mm}(0.25 \mathrm{in.})$

MEAN SLOT DEPTHS: SMITH $4.3 \mathrm{~mm}(0.17 \mathrm{in.})$

CAVIJET: $18.3 \mathrm{~mm}(0.72 \mathrm{in.})$

FIGURE 15 - COMPARISON OF SLOTS CUT BY SELF-RESONATING CAVITATING JET AND CONVENTIONAL JET 
HYDRONAUTICS, Incorporated

APPENDIX C

"The Development of Structured Cavitating Jets for Deep-Hole Bits"

Presented at the

57th Annual SPE Fall Conference

New Orleans, Louisiana

September 26-29; 1982 


\section{THE DEVELOPMENT OF STRUCTURED CAVITATING JETS FOR DEEP-HOLE BITS}

by Virgil E. Johnson, Jr.., Georges L. Chahine, William T. Lindenmuth, Andrew F. Conn, and Gary S. Frederick, HYDRONAUTICS, Incorporated; and George J. Glacchino, Jr.. NL/HYCALOG/NL Industries, Inc.

Copyright 1982, Society of Petroleum Engineers of AME

This paper was presented at the 57th Annual Fall Technical Conference and Exhibition of the Society of Petroleum Engineers of AIME. held in New Orleans. LA. Sept. 26-29, 1982. The material is subject to correction by the author. Permission to copy is restricted to an abstract of not more than 300 words. Write: 6200 N. Central Expressway, P.O. Drawer 64706, Dallas. Texas 75206.

\section{ABSTRACT}

The erosion and cleaning effects of jets are enhanced when the degree of cavitation occurring on or near the bottom of the hole 18 increased. A new, STRuctured Acoustically Tuned Osc11lat1ng Jet ("STRATOJET") system causes the formation of large discrete vortex rings that promote cavitation to depths several times greater than for conventional jets. The new nozrle designs are shown to be sultable for existing mechanical drill bits and may even enhance hole cleaniog in the absence of cavitation. Recent date on the effect of drilling mud on the amplitude of excltation and some laboratory hole bottom cleaning results are also presented.

\section{PREFACE}

During the past seven years, $a$ research and development effort has been conducted on the use of cavitating and structured jets for 1ncreasing the drilling rate of deep-hole mechantcal b1ts. This paper very briefly highlights earlier phases of th1s program, and then discusses some recent efforts to understand the lnfluence of drilling wud on the performance of self-resonating jets and espects for improved bottom hole cleaning. Further detalle on the earlier work can be found in the references 11sted here. Th1s paper describes the current status of an ongoing program, and certaln questions remsin unanswered.

\section{INTRODUCTION}

It 18 generally accepted in the deep-hole drllling Industry that bit hydraulics has a major effect on the rate of penetration. The actual drilling rate 18 always equal to or less than the rate which can be achleved if chips are removed as s00n as they are created 1,2 . Chips are removed from the bottom of the hole by the drilling fluld. since the total discharge through the bit is 11mited by restrictions on the maximum (and mintmum) velocity up the anuulus, the detalls of how this 11mited discharge 18 used to clean the hole bottom whin the confined space avallable within the bit envelope 18 cruclal to the bit performance.

Host roller blts utilize the hydraulic horsepower avallable by ejecting the fluld at the maximum velocity possible from orifices located in the sectors between the roller cones. In many designs these orffices are located at elevations above the hole bottom which are comparable to the cone diameter. The diffusion of these submerged jets is such that the maximum velocity reaching the hole bottom 18 greatly diminished. It has been established ${ }^{3}$ that if the orifices are located closer to the hole bottom by using extension tubes, Improved cleaning and thus increased drilling rates result.

Operating with an overbalance in pressure 18 the normal procedure to resist "blow outs." Thus there 18 alow of fluid into the formation and resulting "filter cake" build-up across the hole bottom. Consequently the fluid passing over the hole bottom to remove ch1ps must " $11 \mathrm{ft}^{\text {" them }}$ agalnst a very large pressure gradient. Th1s holddown force is many times larger than the force caused by gravity.

Clearly any "new" effect that could be ut1I1zed to improve the cleaning action of the jets used in mechanical drill bits wight result in substantial increases in drilling rates. Our background in the mechanics of cavitation and cavitating jets Inltially led us to apply this "new" effect, using existing cavitating nozzle designs. Our recent and ongolng efforts, as described in th1s paper, Involve studies to improve this cavitation effect by seeking passive means of causing the jet to etructure itself into periodic ring vortices. We now confecture that this latter effect may 1mprove clesning of the hole bottom even when the operating conditions are such that cavitation cannot exiat.

In earlier papers (e.8., Ref. 1), the selfresonating nozzle configuration found to be most 
effective for use in drill-bits, to date, was referred to as the "ORGAN-PIPE CAVIJEI"." This nozzle gystem (orifice and feed tube) has now been given the zore descriptive name "STRATOJET," an acronym for STRuctured Acoustically Tuned Orctilating JET, and this new name will be used hereafter in this paper.

\section{BACKGROUND-UNSTRUCTURED CAVITATING JETS}

\section{Cavitating Jet Principles}

The mechanics of cavitating submerged jets and the application of the intense erosion associated with cavitation to a variety of cleaning and cutting applications has been studied at HYDRONAUTICS, Incorporated for over ten years $s^{4-6}$.

The 1mportant parameter defining cavitation is the cavitation number, $\sigma$. For high pressure jets operated at large depths, $\sigma$ is approximately

$$
\sigma \simeq \frac{P_{a}}{\Delta p}
$$

where $P_{a}$ is the amblent or down-hole pressure, and $\Delta p$ is the pressure drop across the nozzle.

The particular value at which cavitation is Inciplent is defined as:

$$
\sigma_{1} \simeq\left(\frac{\mathrm{p}_{\mathrm{a}}}{\Delta \mathrm{p}}\right)_{\text {at inception }} \cdot \cdots \cdot
$$

Thus if the operating conditions for a subwerged jet are such that $\sigma / \sigma_{1}>1$ then cavitation does not occur. When a cavitating jet 18 impinged against a surface, 80 that the cavities formed by the jet are collapsed on that surface, very high pressures (\rangle$\left._{2} \rho V^{2}\right)$ are generated and the resulting cleaning or cutting action is substantially greater than when the jet is not cavitating. As $\sigma / \sigma_{1}$ is reduced below unity the exosion intensity increases. The local dynamic pressure reductions which cause cavitation in a submerged jet formed by a simple contracting nozzle are the result of the eddies created in the shear zone surrounding the jet. The magnitude of these pressure reductions depends on the boundary layer thickness at the nozzle exit. Nozzles we now call "conventional" CAVIJET nozzles have been developed which minimize the boundary layer thickness and thus give maximum velues of $\sigma_{1}$ for a given ratio of supply plpe diameter, $D$, to jet diameter, d.

F1g. 1 presents the approximate inciplent cavItation number, $\sigma_{1}$, as /influenced by the Reynolds number (besed on nozzle diameter and jet velocity) for three types of nozzles. Cavitation for such jets occurs in the shear zone around the jet periphery. These approximate curves represent data obtained from the literature and from tests conducted In our laboratory. The Important points to note in F18. I are the significant differences in $\sigma_{f}$ that exist for the three general classes of nozzles indicated and that the inciplent cavitation index for the conventional CAVIJET nozzle with $D / d=2$ is about the same as for a typical drill bit nozrle. It should also be noted that the higher values of $\sigma_{1}$ achlevable with the conventional CAVIJET require $\mathrm{D} / \mathrm{d}=4$ or greater.

In both laboratory tests with single nozzles, as well as laboratory and fleld trials with twocone roller bits, conventlonal CAVIJET nozzles with $\mathrm{D} / \mathrm{d} \simeq 4$ were found to be more erosive at comparable depths (ambient pressures), and capable of cavitating to greater depths than the other nozzles shown In F1g. 1 (see Refs. 1,7). Space I1mitations, however, In three-cone b1ts, and in other types such as diamond or stratopax bits, usually preclude the use of nozzles with $D / d$ greater than about two. This space limftation, plus the potential for greatly increasing $\sigma_{i}$, and hence the depth at which cavitation would occur, were the two specific motivations for our investigation of selfexclted resonating nozzles.

\section{STRUCTURED JETS - THEORY AND CONCEPTS}

Effects of Jet Structuring on Cavitation Inception

In Ref. 8, Crow and Champagne reported the remarkable change in the structure of an air jet when its driving pressure was excited at certain frequencies by a loud-speaker $s 0$ as to produce an oscillation in the exit velocity of only a few percent. The frequency, $f$, required for essentially complete structuring of the jet into discrete vortices was defined by a strouhal number, $S_{d}=f d / v$, of approximately 0.3 for the nozzle used (where $d$ and $v$ are the nozzle diameter and the jet velocity respectively). We recognized that the phenomenon described by Crow and Champagne should have an application in Improving the performance of cavitating submerged jets if the excitation provided by the loud-speaker could be somehow passively provided in a feedback oscillator system. The results of our initial studies into the fessibility of such a scheme are reported in Ref. 9 .

Analyses 1 which compared the performance of structured and nonstructured jets have indicated why a self-resonating nozzle has the potential of Improving drill bit performance. It has been shown that the $\sigma_{1}$ for a structured jet is larger, relative to a nonstructured jet, both for free flow and when the jets Implinge upon a surface (see F1g. 2). As discussed below, the low pressure induced on the boundary will be gignificant in cleaning the bottom of deep holes drilled with mechanical bits which Incorporate jets structured into discrete vortices.

The STRATOJET Concept for Achieving a Passively Structured Jet

In Ref. 9 several methods of passively achlevIng fet pulsation were shown to be feasible. Our recent work has concentrated on the STRATOJET concept shown In Fig. 3 because of Its simplicity and because it shows the most promlse of belng adaptable to existing mechanical drill bits with only minor changes to the hydraulic passages in the bits.

The principles involved in the STRATOJET concept are common to any passive resonating system. A jet forming nozzle is fed from a supply system that has a natural acoustic frequency equal to the preferred frequency in the turbulence spectrum of the jet. The jet forming nozzle must be 
shaped to feed back to the supply system pressure osclllations associated with the forwiation of ring vortlces in the structured jet. The fed-back oscillations cause resonance to occur in the supply chamber which in turn further excites the fet until the dampling in the system causes equilibrlum to be reached. Although simple in concept, detalled dee1gn of an optimum system is quite complex-thus a quasi-empirical approach has been used to develop atrongly resonsting nozzles. However, the general approach for developing this design is as follows.

The STRATOJET concept shown In F1g. 3 should achieve peak acoustic resonance when a standing wave forms in the "organ-pipe" section (length: $L_{p}$. diameter: D). Th1s section 18 created by the upatream contraction; $\left(D_{g} / D\right)^{2}$ and the nozzle contraction $(D / d)^{2}$. Peak resonance 111 occur when the frequency of the organ-plpe wave is near the critical jet structuring frequency, as given by the nozzle strouhal number, $s_{\mathrm{d}^{*}}$, but the exact resonance 18 dependent on the contractions at each end of the organ-pipe. For instance, if both $\left(D_{s} / D\right)^{2}$ and $(D / d)^{2}$ are large, then the first mode resonance in the pipe will occur when the sound wave length in the fluid is approximately four tImes $L_{p}$ - Acoustic analyses and experimentation have led to the following approximation, useful for estimating the length of the organ-pipe:

$$
\frac{L_{p}}{d} \simeq \frac{K_{p}}{M S_{d}^{*}}
$$

where the "mode parameter", $K_{n} 18$ given by:

$$
\begin{array}{r}
K_{n}=F\left\{n,\left(\frac{D_{s}}{D}\right)^{2},\left(\frac{D}{d}\right)^{2}\right\} \\
\simeq \frac{2 n-1}{4} \text { for }\left(\frac{D_{s}}{D}\right)^{2} \text { and }\left(\frac{D}{d}\right)^{2} \gg 1 / \sqrt{M} . \\
\simeq \frac{n}{2} \text { for }\left(\frac{D_{s}}{D}\right)^{2} \gg 1, \text { but }\left(\frac{D}{d}\right)^{2} \leq 1 / \sqrt{M} .
\end{array}
$$

In these expressions:

$n=$ mode number of the organ-pipe,

$s_{d}^{*}=$ critical Strouhal number, $f d / v=0.3$

to 0.8

$$
M \text { - Mach number, } V / c \text {. }
$$

other STRATOJET concepts found effective had stepped variations in the organ-pipe section diameter. By locating the steps (d1ameter-changes) at desired pressure node sites, it 1s possible to "force" a particular mode, n. Including such steps also provides a means of mintmizing the hydraulic pressure loss in the tube consistent with structural considerations.

Let us assume that the STRATOJET concept 18 to be applied to an existing extended nozzle threecone bit which 18 to be operated over a prescribed range of pressure drops. The overall length of the tube is fixed. During drilling, several nozzle sizes are required in order to maintain discharge over the range of pressure drops. As shown below, the tuned "width" (1.e., the width or range of Mach numbers in a resonance plot wuch as F1g. 5) for etrong oscillations of good STRATOSET designs is about \pm 15 percent of the design Kach number or about $\mp 30$ percent of the design pressure drop across the nozzle (for a given fluld density). This resonant peak width provides an adequate de1gn range for most practical applications. For example, if the nozzle is designed for peak performance at pressure drop across the nozzle of $1,500 \mathrm{psi}(10.3 \mathrm{MPa})$, structuring of the jet, and hence resonance, may be expected over a range of $1,000 \mathrm{psi}<\Delta \mathrm{p}<2,000 \mathrm{ps1},(6.9 \mathrm{MPa}<\Delta \mathrm{p}<13.8$ MPa).

Our effort has primarily been devoted to finding nozzle designs which, given a prescribed nozzle exit diameter and Mach number (pressure drop), have the required $S_{d}{ }^{*}$, and provide optimum resonating amplitudes.

\section{STRUCTURED JET EXPERIMENTS}

\section{Experimental Fac1lities}

Two facllities were developed to study the characteristics of self-resonating jets. An air facllity allowed examinations of jet performance with the capability of rapid and economical changes in a design iteration that were not 80 readily made in a high-pressure water jet fac1lity. Although the alr facility was operated at Reynolds numbers which were too low to allow complete scalIng to the water results, valuable insights which guided and minimized the more tedious tests in water were gained by studies of resonating alr jets.

A1r for the nozzle belng tested was fed through a large, rectangular plenum. Both free and impinging jets were studied, using a hot film sensor to monitor flow perturbations. A spectrum analyzer, an oscllloscope, and an $x-y$ plotter were used, respectively, to process, observe, and record the test results from the alr facllity as well as from the water tests in the high pressure cell (HPC). Thls cell, which can contaln internal presBures up to $3,000 \mathrm{psi}$, was used for pressure osc1llation studies, visual observations of jet structure, and rock cutting tests. Rock specimens, typically cubes 6 in. on a side, can be rotated beneath the jet In the HPC, at rates up to 66 rpm by means of a varlable speed drive. For flow visualization and photography, the BPC has been fitted with three circular viewing ports, each 1.5 in. In diameter. Plezoelectric pressure transducers mounted in the inlet feed pipe and in the cell wall monitored pressure fluctuations.

\section{Jet Structuring Observations}

Well over a hundred different organ-plpe configurations have been Investigated, both in air and in water. Most were stralght although some utilized the actual curved extended tubes from SMITH roller bits, and tests were also conducted, in air, with a complete 12-1/4 1n. diameter SMITH F2 three-cone bit. Runs were also made in water using a one-third sector of such a bit. In most of the tests conducted, the observed resonant frequency was within about 10 percent of the frequency which corresponds to multiples of a half wave length. Such a result is expected because the nominal 
supply pipe to orifice diameter in all of the tests conducted was two or less. Thus, good agreement was found 1 between the experimental observations and the trends predicted by equation ( 3 ).

F1g. 4 illustrates the nozrle features which were varied 80 as to determine their effect on the critical Strouhal number. F1g. 5 shows the measured response in water of a fixed organ-pipe cupply tube and nozzle with fixed inlet contour. The different curves are for various throat lengths and exit contours. The oscillating Strouhsl numbers for each nozzle are also shown in this figure. It 18 important to note that there is a substantial variation in the critical Strouhal number as the nozzle features are changed. We have tested nozzles having $S_{d}^{*}$ as low as 0.28 and as high as 0.9. As typlfied by the results in Fig. 5 , the width of the response curve 18 about \pm 15 percent of the Nach number at which maximum modulation amplitude occurs. It is also seen that this maximum amplitude decreases as $S_{d}$ is increased-a result anticlpated from analyses 1 of the effect of vortex structure on jet modulation.

It was Important to be able to vary the critical strouhal number by changing the nozzle features, so that self-excited systems could be designed despite constralnts on the tube length. However, when there are no length restralnts, nozzles should be selected to have the lowest possible $\mathrm{S}_{\mathrm{d}^{\star}}$, compatible with jet structuring, so that the clrculation strength of each vortex is maxim1zed.

The prediction that structured fets should have higher incipient cavitation numbers in comparison to unstructured jets was confirmed by every test conducted in water. In most cases, where the jet 1mpacted on a solld wall, cavitation was first observed near the boundary, and out several jet radil from the jet axis. Using the curve shown in F18. 1 for conventional drill bit nozzles as a bench mark for inciplent cavitation numbers, the measured incipient cavitation numbers for the selfexcited structured jets tested were two to $81 x$ times higher than this baseline curve. As stated, almost all of the nozzles studied had supply plpe to orifice diameter ratios of two or less. Thus the major goal of achleving higher inclplent cavitation numbers for nozzles supplied with pipe diameters less than approximately twice the orifice diameter has been achleved.

A further observation has been made relative to cavitation. It has been seen that many nozzle shapes are capable of self excitation when the operating cavitation number is less than the inception value. However, when $\sigma / \sigma_{i}>1$ only a narrow family of nozzle shapes will self excite and structure into vortex rings. Further research is required to explain this phenomenon.

\section{Rock Cutting Tests}

Although most of our work has been directed toward developing procedures for designing selfexcited structured jet nozzles to be tried in existing mechanical bits, we have carried out several tests to compare the rock cutting abllity of unstructured and structured cavitating jets. In comparison, between ORGAN-PIPE CAVIJET nozzles and nozzles of the type that are typically supplied for use in roller-cone bits, the depths of slots cut were typically two to four times greater for the structured jet. Typical results are shown in Table 1.

\section{HOLE BOTTOM CLEANING}

\section{Effects of Jet Structuring}

It has been shown ${ }^{1}$ that a structured jet should produce an instantaneous pressure distribution on a boundary as shown, approximately, in F18. 6. The pressures $\mathrm{Pa}_{a}$ and $\mathrm{Pb}$ are the amblent pressure and boundary pressure, respectively. The cross hatched rectangles represent constant value pulses of width, $w$, that approximate the actual pressure distribution. Although it is recognized that the vortex spacing will alter as the rings approach and spread over the boundary, the assumption that this spacing is constant is not crucial to the general magnitude of the minimum pressures that w111 occur on the boundary; hovever, the relative locations may be different. Viscous effects will also modify the results shown in Pig. 6 . Obviously friction and vortex break-down should have a large influence, particularly when $r / d>1$. However, as long as the vortices do not break down, unusually high theoretical negative instantaneous pressures must occur on the boundary-an order of magnitude greater than the nozzle pressure drop. Compare th1s with the turbulent fluctuations of an unstructured jet which are generally about an order of magnitude less than the nozzle pressure drop. If such large pressure fluctuations do result when a structured fet impinges on a boundary, they may be large enough to lift small chlps agalnst the pressure gradient forces at the hole bottom as discussed in the Introduction section. A schematic drawing of the various pressures acting on a chip is shown in FIg. 7. Preliminary analyses 1 have shown that ample chip lifting pressures should be generated by the mechanism indicated in F1g. 6 .

\section{Experimental Results}

In order to confirm the improved cleaning ability of a STRATOJET system as compared with a standard extended tube-nozzle system, a new low pressure ch1p hold-down facllity was constructed (F1g. 8) and some preliminary results have been obtained. The clear plastic test cell has an 11 in. I.D., and may be pressurized to about 165 psia. Flow across the nozzle can be varled up to $65 \mathrm{gpm}$, with nozzle pressure drops, $\Delta p$, up to $75 \mathrm{psi}$ for the $0.5 \mathrm{ln}$. dlameter used. High speed photography and pressure fluctuations measurements have confirmed, qualitatively, the concept shown in Fig. 6. Large negative pressure spikes have been recorded for the STRATOJET, with amplitudes that are approximately the same order of magnitude as $\Delta p$.

To perform chip lifting experiments, the test cell is given a porous, sintered-metal, stalnless steel base. Upon this base, varlous filter screens are placed, and a filter cake of drilling mud is allowed to form. Typical results are shown in F1g. 9; for this test a nylon wesh cloth with maximum pore size of $5 \mu$ was used, although a variety of nylon and metal wesh screens haye been examined. The nominal porosity of this $5 \mu$ cloth was 0.01 (ratio of calculated total pore area to total 
area). Tests were conducted by first allowing the filter cake to bulld, clogging the filter pores, unt11 a table "hold-down pressure", $\Delta \mathrm{p}_{\mathrm{p}}$, was established. The nozzle pressure drop, $\Delta \frac{h}{p}$, was then gradually Increased unt1l a sudden drop was observed in the hold-down pressure-signifying chip-iffting and a penetration of the mud cake.

For the tests in Fig. 9, mud particle sizes ranged from 2 to $5 Q_{1}$, with maximum volume concentration at about $8 \%$. As seen in this figure, the STRATOJET system was three to four times more effective in causing chip lifting, relative to a standard drill-bit nozzle.

\section{SCALE AND FLUID PROPERTY EFFECTS}

The abllity to conduct meaningful small size laboratory studies of STRATOJET gystems requires an understanding of scaling aspects of the resonating phenomenon. Also, as discussed above, it has been convenlent to use afr and water, instead of mud, to develop these fets. Th1s section describes the current state of understanding of scale and fluid property effects, and the ongolng studies to design. nozzle systems for field trials.

\section{Feedback Mechanisms}

An unsteady flow separation and reattachment process within the STRATOJET nozzle orffice interacts with the flow 1mmediately downstream of the nozzle exit. This interaction, if caused to occur at the critical strouhal number, provides the feedback mechanisms which create the strons resonance in these jets. Th1s phenomenon can be scaled-if tests are run with geowetrically simflar STRATOJET nozzles, at the same Mach number and Reynolds number-as our experiments have demonstrated.

Scaling between two Newtonlan fluids, such as ait and water, 18 also possible. If, however, the fluld is not Newtonian, such as drililing mud, then rheological effects will change each part of the feedback mechanlsms (flow separation, reattachment, and subsequent shedding into ring vortices) which control the self-resonance. For this reason, nozzle configurations providing strong tesonance in air and water w11l not produce the same fluctuating amplitude at the same Mach number (nozzle pressure drop) In mud. An adequate theoretical model of the feedback mechenisms of STRATOJET systems has yet to be formulated. Thus, an experimental effort, comparable to that exerted with air and water, must be completed to develop opt lmum mud-designs for STRATOJET systems.

\section{Preliminary Mud Tests}

An Initial series of tests to examine mud effects was conducted the Drilling Research Laboratory (DRL), December 1981 and January 1982. We used a mocified SMITH TOOL, 12-1/4 in. F-3 threecone bit with a STRATOJET system, consisting of redesigned extended tubes and nozzles developed from our earlier alr and water studies. Prior atr testing with this complete bit had shown strong ofkillations in the as-designed range of Mach numbers $(0.08$ to 0.1$)$, which correspond, for a $9.51 \mathrm{~b} / \mathrm{gal}$ mud, to a $\Delta \mathrm{p}$ range of about 1,000 to $2,000 \mathrm{ps} 1$. single-nozzle, 40 percent scale model tests of this
STRATOJET system, in water and in water with polymer ( 1 and $2 \mathrm{lb} / \mathrm{bbl}$ solutions of Relzan), showed comparable strong oscillations in the design range.

At DRL, resonance was observed by placing plezoelectric transducers in both the feed and choke IInes of the Well Bore SImulator. With water, the full-acale bit produced a comparable strong resonance to that prevlously seen for the single, 40 percent scale STRATOJET. For example, at Mach number, $M=0.09(\Delta p=1500$ psi), the modulation $\left(p^{\prime} / \Delta p\right)$ in the feedplpe was about $B$ percent, at about an $8 \mathrm{kHz}$ frequency of oscillation. As anticipated, with \& $9.5 \mathrm{lb} / \mathrm{gal}$ waterbased mud, both the amplitude and Mach number for peak resonance were affected. At the off-peak Mach number of $0.09, p^{\prime} / \Delta p$ was only about 0.5 percent. The peak modulation in mud was at $M=0.07$ $\left(\Delta p=1,000\right.$ psi), with $p^{\prime} / \Delta p \simeq 1.6$ percent.

Comparative drilling tests were then performed in Mancos shale, using a standard SMITH F2-CE as the baseline bit. The range of $\Delta p$ was 1,000 to 1,750 psi; ambient pressures were varied from 800 to $2,500 \mathrm{psi}$. At the highest $\Delta \mathrm{p}$ value, where minimal modulation occurred $\left(p^{\prime} / \Delta p \simeq 0.2\right.$ percent) the drilling rate normallzed with discharge was approximately the same for the standard and the modifled b1ts. For the 1,000 psi nozzle pressure drop (where $p^{\prime} / \Delta p$ was approximately 1.6 percent), the absolute drilling rate was 10 percent higher for the modified bit, despite a discharge approximately 15 percent less. However, when normalized by the respective discharges, the $\Delta p=1,000$ psi drilling rate was nearly 30 percent higher with the STRATOJET. All of the data showed a general relative increase in drilling rate as $p^{\prime / \Delta p}$ increased from 0.5 to 1.6 percent. Thus the test results were encouraging in that they indicated a potential large increase in drilling rate if the amplitudes of oscillation obtalned in water could be achleved in drilling mud. We are now engaged in a development program designed to accomplish strong resonance in drilling mud.

\section{CONCLUDING REMARKS}

Since any report of a work in progress must contain many unanswered questions, we beg the reader's indulgence and trust that continued efforts now being exerted will allow us to fill in these gaps.

However, several 1mportant conclusions are now possible and may be summarized as follows:

1. Using the STRATOJET concept, self-excited jets can be obtalned which structure themselves into perlodic ring vortices. This concept is adaptable for utilization in mechantcal bits used in current deep-hole drilling.

2. STRATOJET systems have measured inciplent cavitation numbers two to six times higher than those obtalned for conventional drill bit nozzles. Thus, bits utilizing self-resonating cavitation effects will have drilling rates influenced at depths two to six times greater than with tools now belng used.

These increased values of inciplent cavitation numbers are consistent with theoretical pre- 
dictions and give some credence, to the possibility that such structured jets should produce lmproved bottom hole cleaning, even when operating at depths below where cavitation will exist.

3. Preliminary chip hold-down experiments conducted in a laboratory test loop show that STRATOJET nozzle systems clean chips held down by a pressure gradient with a nozzle pressure drop only about 25 percent of that required for standard nozzle systems.

4. The measured natural frequencles of the organpipe systems tested correspond to multiples of half wave length standing waves.

5. The response width of self-excited systems is epproximately \pm 30 percent of the design nozzle pressure drop.

6. The critical strouhal number may be varied by changing detalls of the nozzle geometry. Thus it Is possible to design practical organ-pipe systems for many specifled operating conditions.

7. Some nozzle designs which self exclte under cavitating conditions do not continue to do 80 under noncavitating conditions. However, designs have been tested which self excite and produce structured jets regardless of the operating cavitation number.

8. There are 1mportant differences in the critical Strouhal number and amplitude of oscillation, of nozzles tested in air, water, and mud.

\section{ACKNOWLEDGEMENTS}

The work described in this paper was supported in part by the U.S. Department of Energy, Division of Geothermal Energy, under Sandia Laboratories Contract Nos. 13-5111 and 13-5129; support by the authors' companles, HYDRONAUTICS, Incorporated and NL HYCALOG/ML Industries, Incorporated has also been provided for this study. We also want to acknowledge the participation of Sil SMITH TOOL in the testing at DRL.

\section{REFERENCES}

1. Johnson, V.E., Jr., et al., "Cavitating and Structured Jets for Mechanical Bits to Increase Drilling Rate," ASME Paper No. 82-Pet-13, presented at the Energy Technology Conference and Exhibition, New Orleans, March 7-11, 1982.
2. Maurer, W.C., "The 'Perfect-Cleaning' Theory of Rotary Driliing," Journal of Petroleum Technology, 1962, pp. 97-101.

3. Feenstra, R. and Van Leeuwen, J.J.M., "Full Scale Experiments on Jets in Impermesble Rock Dr1lling," Journal of Petroleum Technology, vol. 231, March 1964, Pp. 329-336.

4. Johnson, V.E., Jr., et al., "Tunnell1ng, Fracturing, Drilling and Mining with High Speed Water Jets Utilizing Cavitation Damage," Proc. F1rst Int'1. Sympos. on Jet Cutt1ng Technology (Coventry, U.K.: April 5-7, 1972) Cranfield, U.K., BHRA Fluld Englneering, Paper A3, 1972, 19 pp.

5. Conn, A.F. and Rudy, S.L., "Conservation and Extraction of Energy with the CAVIJET", " Proc. Fourth Int'1. Sympos, on Jet Cutting Technology (Canterbury, U.K.: AprII 12-14, 1978) Cranfleld, U.K., BHRA Fluid Englneering, Vol. 1, Paper H2, 1978, 20 pp.

6. Conn, A.F. and Johnson, V.E., Jr., "The Fluid Dynamics of Subierged Cavitating Jet Cutting," Proc. Fifth Int'1. Sympos. on Jet Cutting Technology (Hanover, F.G.R.: June 2-4, 1980), Cranfield, U.K., BHRA Fluld Englneering, Paper A1, 1980, pp. $1-14$.

7. Conn, A.F., Johnson, V.E., Jr., LIU, H.L., and Frederick, G.S., "Evaluation of CAVIJET $\otimes$ Cavitating Jets for Deep-Hole Rock Cutting," HYDRONAUTICS, Incorporated Technical Report 7821-1. August 1979 (Also Sand 1a Laboratories Contractor Report SAND81-7067, May 1981).

8. Crow, S.C. and Champagne, F.H., "Orderly Structure in Jet Turbulence," Journal of Fluid Mechanics, Vo1. 48, Part 3, August 1971, Pp. 547-591.

9. Johnson, V.E., Jr., Lindenmuth, W.T., Conn, A.F., and Frederick,G.S., "Feasibility Study of Tuned-Resonator, Pulsating Cavitating Water Jet for Deep-Hole Drilling," HYDRONAUTICS, Incorporated Technical Report 8001-1, May 1981 (also Sand1a Laboratories Contractor Report SAND817126, August 1981).

\section{Table 1 Slot cutting comparisons}

NOZZLES: Conventional SMITH. $d=0.170 \mathrm{in} .(4.3 \mathrm{~mm})$ ORGAN-PIPE CAVIJET, $d=0.185$ in. $(4.7 \mathrm{~mm})$ ROCK: Indiana limestone; STANDOFF: $x / d=3$ TRANSLATION VELOCITY: $0.25 \mathrm{in.} / \mathrm{s}(6.4 \mathrm{~mm} / \mathrm{s})$

\begin{tabular}{|l|c|c|c|}
\hline $\begin{array}{c}\text { Pressure, } \\
\text { Nozzle } \\
\Delta p . \text { psi (MPa) }\end{array}$ & $\begin{array}{c}\text { Ambient } \\
\text { Pressure, } \\
\mathrm{p}_{\mathrm{a}}, \text { Psi (MPa) }\end{array}$ & $\begin{array}{c}\text { Cavitation } \\
\text { Number, } \\
\sigma\end{array}$ & $\begin{array}{c}\text { Mean Slot } \\
\text { Depth Ratio. } \\
\text { CAVIJET } \\
\text { SMITH }\end{array}$ \\
\hline \multicolumn{4}{|c|}{ SINGLE PASS } \\
\hline $2.530(17.4)$ & $170(1.2)$ & 0.067 & 1.16 \\
$2,500(17.2)$ & $600(4.1)$ & 0.24 & 2.54 \\
$2,500(17.2)$ & $1,200(8.3)$ & 0.48 & 2.33 \\
\hline \multicolumn{5}{|c|}{ TWENTY-FIVE PASSES } \\
\hline $1.225(8.4)$ & $325(2.2)$ & 0.27 & 1.90 \\
\hline
\end{tabular}




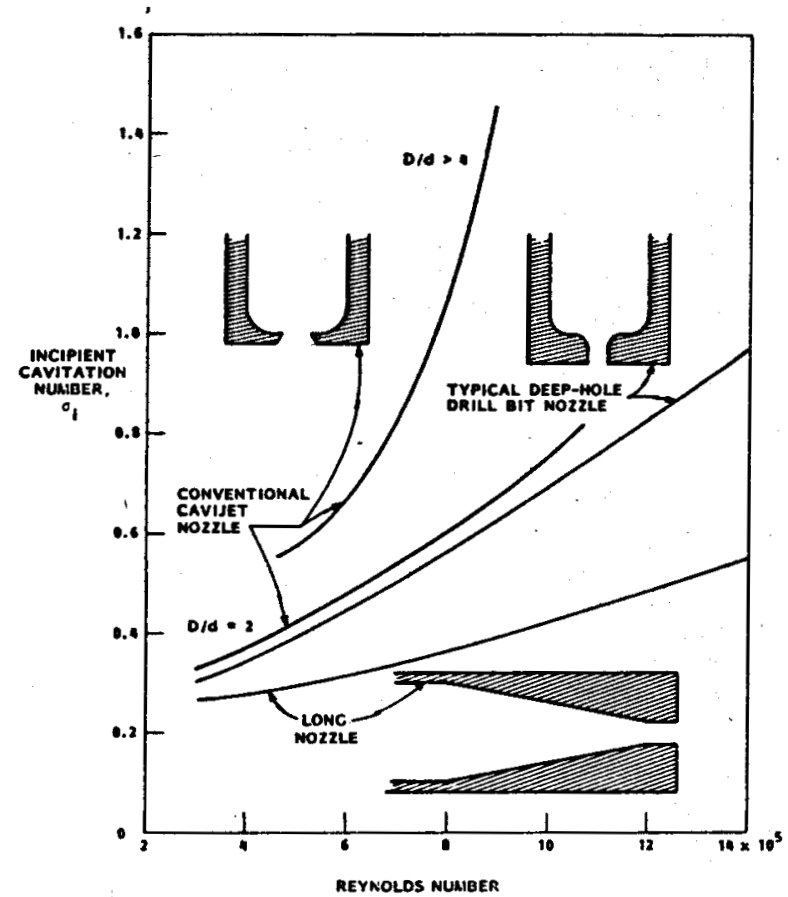

Fig. 1 - Approximate incipient cavitation numbers for CAVIJET ${ }^{\infty}$ and other nozzle types (Acoustic detection)

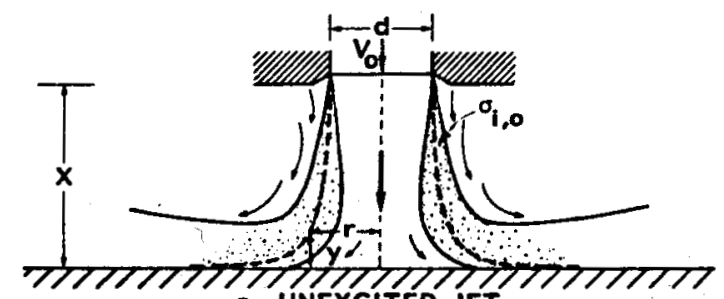

a. UNEXCITÉ JET

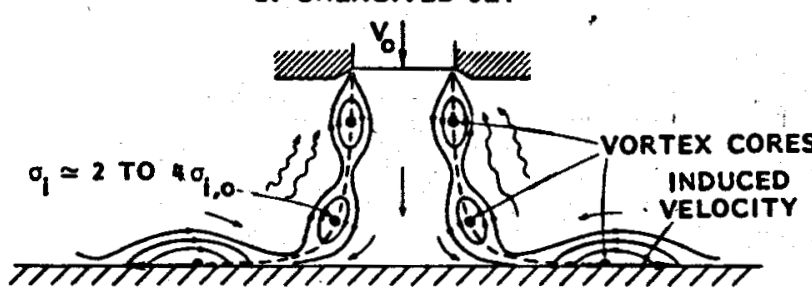

b. EXCITED JET

Fig. 2 - Flow comparison of impinging submerged cavitating jets

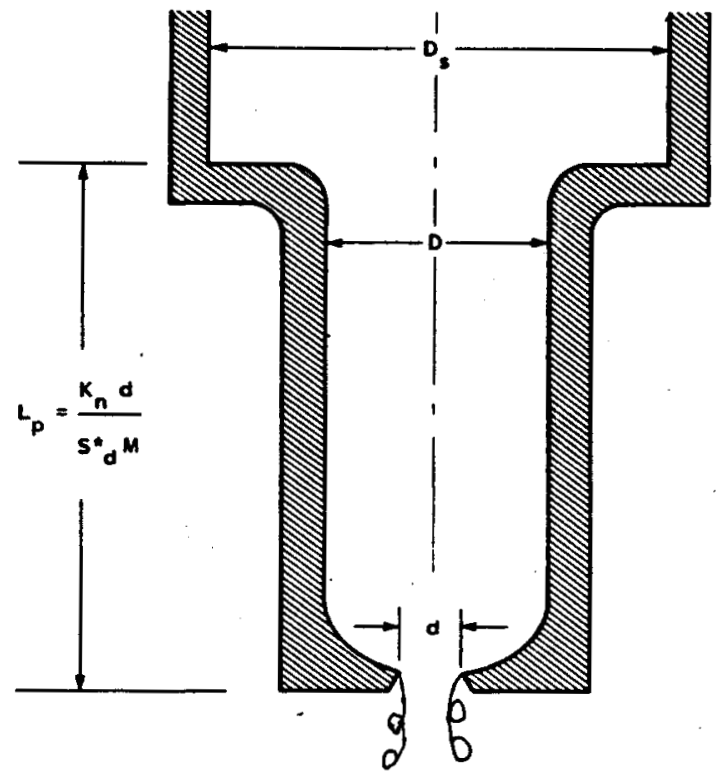

Fig. 3 - STRATOJET nozzle concept

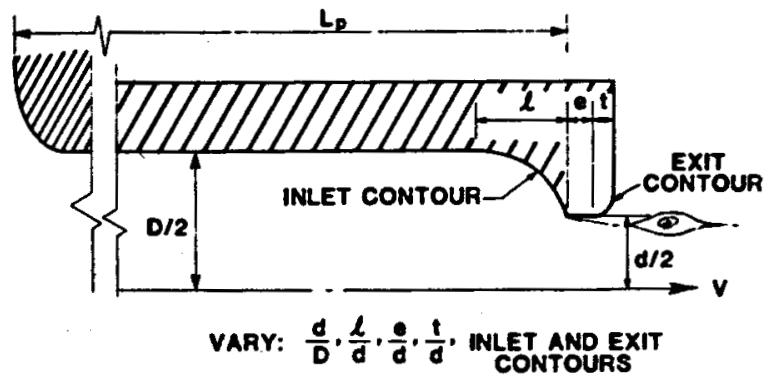

Fig. - STRATOJET nozzle features tested to determine effect on critical Strouhal number

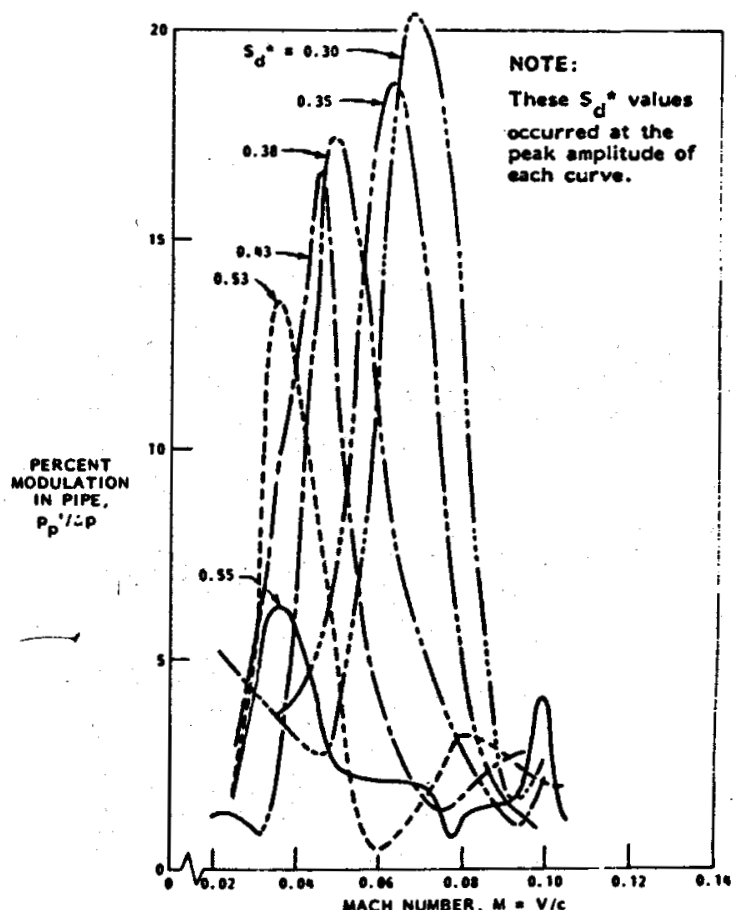

Fig. 5 - Effect of variations in nozzle shape on STRATOJET resonance in water. Nozzle diameter: $d=0.20$ in.; Feed-tube: straight, diameter: $D=0.40 \mathrm{in.}$. length: $L_{p}=4.37 \mathrm{in}$. 

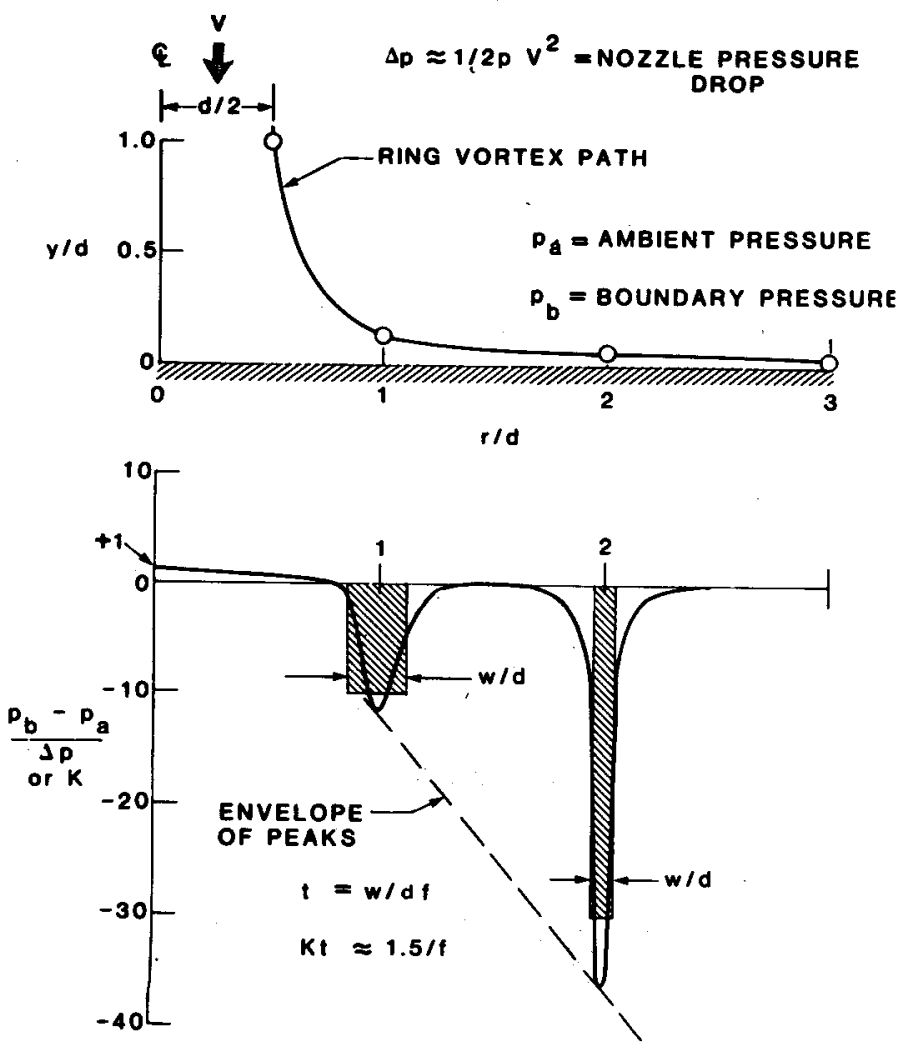

Fig. 6 - Instantaneous boundary pressures for structured jet

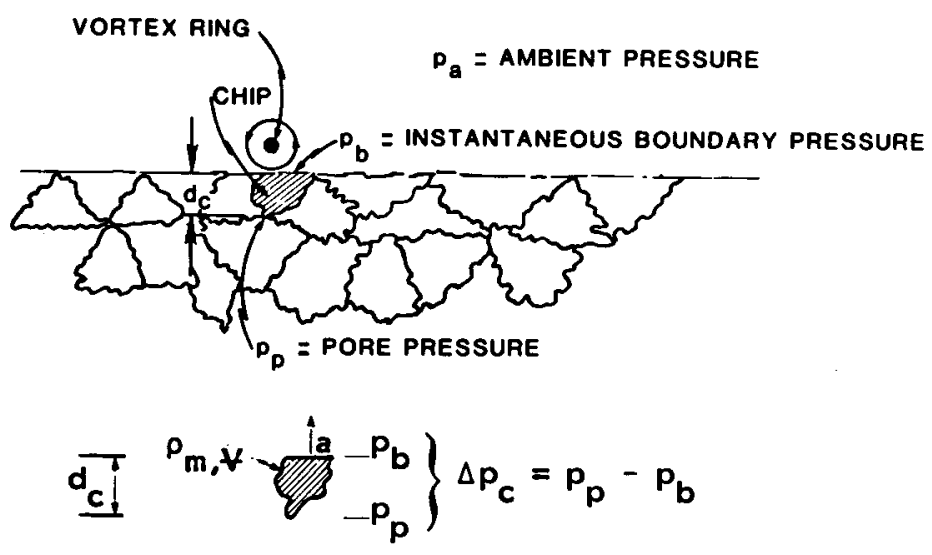

Fig. 7 - Chip hold-down

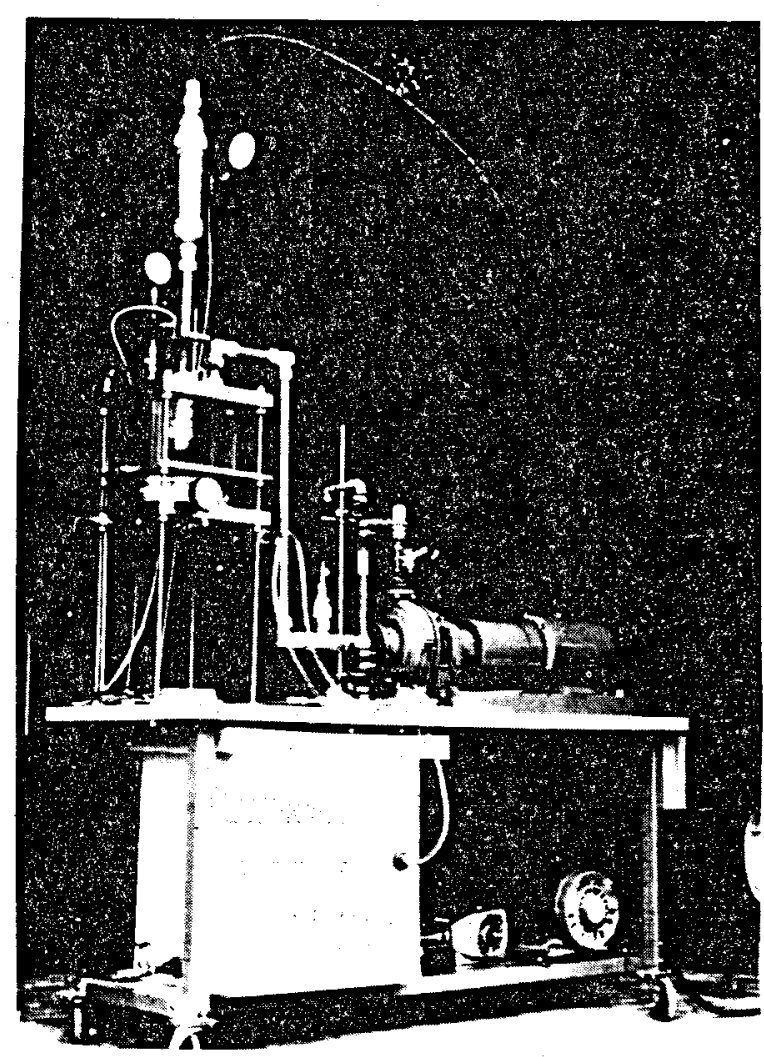

Fig. 8 - Chip hold-down test loop

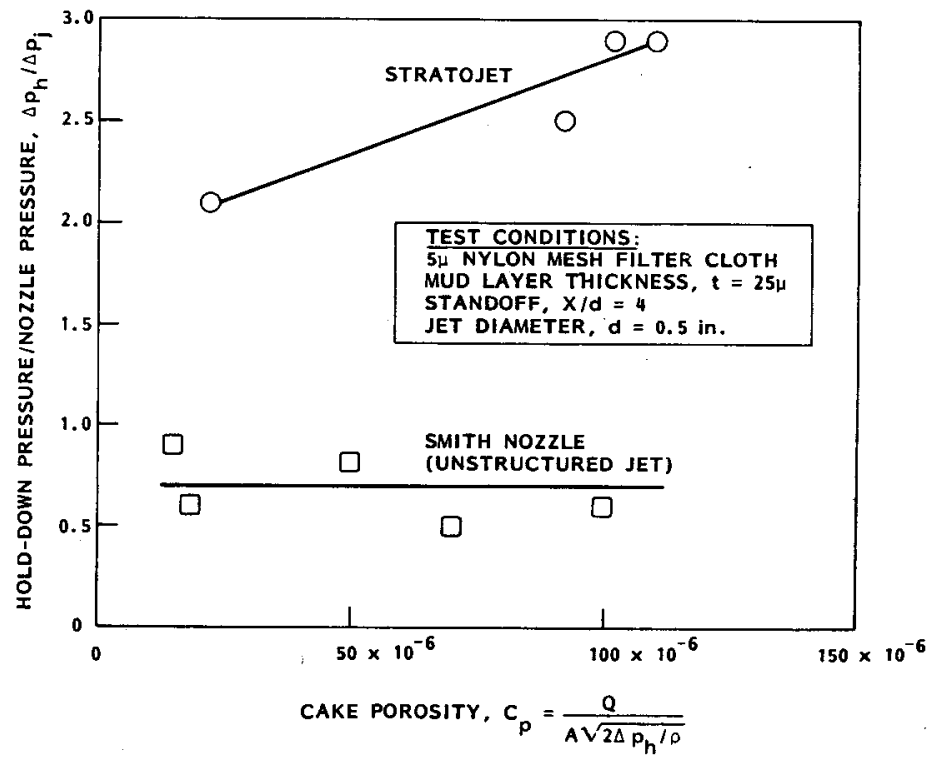

Fig. 9 - Comparison of mud cake penetration for unstructured and structured jets 
DISTRIBUTION:

TID-4500-R66-UC-66c (474)

Tom Anderson

Drilling Fluid Consultants 17726 S.W. Overlook Ln.

Lake Oswego, OR 97034

Ed Bingman

Shell Oil Company

Two Shel1 Plaza

P.0. Box 2099 .

Houston, TX 77001

Larry Dianond

Dyna-Dri11

P.0. Box C-19576

Irvine, CA 92713

Ton Turner

Phillips Petroleum Company

Geothermal Operations

655 East 4500 South

Sa1t Lake City, UT 84107

Jim Kingsolver

Geothermal Operations

Smith Tool

P.0. Box C-19511

Irvine, CA 92713

John C. Rowley

Los Alamos National Labs

Mail Stop 570

Los Alamos, NM 87545

Ed Martin

Superior 0il

Eastern Division

P.0. Box 51108 OCS

Lafayette, LA 70505

B. J. Livesay

129 Liverpoo1

Cardiff, CA 92007

Ben Bradford

Dowell

P.0. Box 2710

Tulsa, OK 74102

Gene Polk

NL Baroid

P.0. Box 280

Sandia Park, NM 87047

James W. Langford

Security Division

Dresser Industries, Inc.

P.0. Box 24647

Dallas, TX 75224

John E. Fontenot

NL, MWD

P.0. Box 60070

Houston, TX 77205

De1 E. Pyle

Union Geothermal Division

Union 0il Co. of California

Union Oil Center

Los Angeles, CA 90017

William D. Rumbaugh

Research \& Development

Otis

P.0. Box 34380

Dallas, TX 75234

Dwight Snith

Halliburton

Drawer 1431

Duncan, OK 73533

Tom Warren

Amoco Production Company

Research Center

P.0. Box 591

Tulsa, OK 74102

H. E. Mallory

P.0. Box 54696

Tulsa, OK 74144

Jim Combs

Geothermal Resources Int'1. Inc. 545 Middlefield Rd., Suite 200 Menlo Park, CA 94025 
DISTRIBUTION cont.

Dr. Melvin Friedman

Dept. of Geology

Texas A\&्qM University

College Station, TX 77843

Virgil Johnson (5)

Tracor Hydronautics

7210 Pindell School Rd.

Laure1, MD 20707

A. Adduci

U.S. Department of Energy

San Francisco Operations Office

1333 Broadway

Wells Fargo Building

Oakland, CA 94612

U.S. Department of Energy (3)

Geothermal Hydropower

Technologies Division

Forrestal Bldg., CE 324

1000 Independence Ave. S.W.

Washington, D.C. 20585

Attn: J. Bresee

R. Toms

D. A11en

W. P. Grace, DOE/ALO

Nuclear \& Geosciences Division

3141 L. J. Erickson (5)

3151 W. L. Garner (3)

6200 V. L. Dugan

6240 R. K. Traeger

6241 J. R. Kelsey (10)

6241 D. A. Glowka (10)

6246 B. Granoff

6247 P. J. Hommert

6250 B. W. Marsha11

6330 W. D. Weart

8214 M. A. Pound 